\title{
Water-Quality, Water-Level, and Discharge Data Associated with the Mississippi Embayment Agricultural Chemical Transport Study, 2006-2008
}

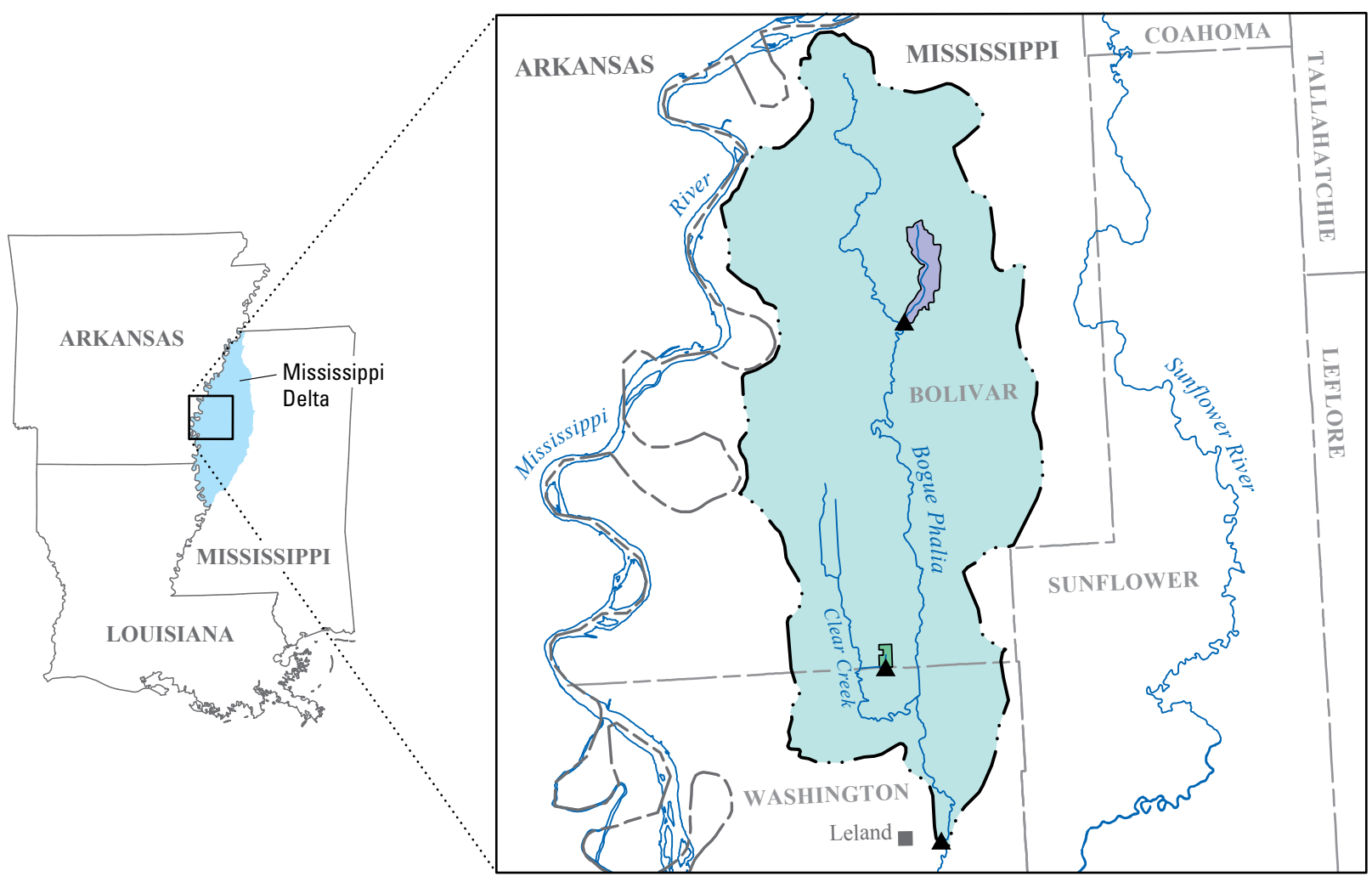

Data Series 546 
Cover. See figure 1 of this report. 


\section{Water-Quality, Water-Level, and Discharge Data Associated with the Mississippi Embayment Agricultural Chemical Transport Study, 2006-2008}

By Melinda S. Dalton, Claire E. Rose, and Richard H. Coupe

Data Series 546 


\section{U.S. Department of the Interior \\ KEN SALAZAR, Secretary \\ U.S. Geological Survey \\ Marcia K. McNutt, Director}

\section{U.S. Geological Survey, Reston, Virginia: 2010}

For more information on the USGS - the Federal source for science about the Earth, its natural and living resources, natural hazards, and the environment, visit http://www.usgs.gov or call 1-888-ASK-USGS

For an overview of USGS information products, including maps, imagery, and publications, visit $h$ ttp://www.usgs.gov/pubprod

To order this and other USGS information products, visit http://store.usgs.gov

Any use of trade, product, or firm names is for descriptive purposes only and does not imply endorsement by the U.S. Government.

Although this report is in the public domain, permission must be secured from the individual copyright owners to reproduce any copyrighted materials contained within this report.

Suggested citation:

Dalton, M.S., Rose, C.E., and Coupe, R.H., 2010, Water-quality, water-Level, and discharge data associated with the Mississippi Embayment Agricultural Chemical Transport study, 2006-2008: U.S. Geological Survey Data Series 546, $60 \mathrm{p}$. 


\section{Foreword}

The U.S. Geological Survey (USGS) is committed to providing the Nation with reliable scientific information that helps to enhance and protect the overall quality of life and that facilitates effective management of water, biological, energy, and mineral resources (http://www.usgs.gov/). Information on the Nation's water resources is critical to ensuring long-term availability of water that is safe for drinking and recreation and is suitable for industry, irrigation, and fish and wildlife. Population growth and increasing demands for water make the availability of that water, measured in terms of quantity and quality, even more essential to the long-term sustainability of our communities and ecosystems.

The USGS implemented the National Water-Quality Assessment (NAWOA) Program in 1991 to support national, regional, State, and local information needs and decisions related to water-quality management and policy (http://water.usgs.gov/nawqa). The NAWOA Program is designed to answer: What is the quality of our Nation's streams and ground water? How are conditions changing over time? How do natural features and human activities affect the quality of streams and ground water, and where are those effects most pronounced? By combining information on water chemistry, physical characteristics, stream habitat, and aquatic life, the NAWQA Program aims to provide science-based insights for current and emerging water issues and priorities. From 1991 to 2001, the NAWQA Program completed interdisciplinary assessments and established a baseline understanding of water-quality conditions in 51 of the Nation's river basins and aquifers, referred to as Study Units (http://water.usgs.gov/nawqa/ studies/study_units.htm/).

In the second decade of the Program (2001-2012), a major focus is on regional assessments of water-quality conditions and trends. These regional assessments are based on major river basins and principal aquifers, which encompass larger regions of the country than the Study Units. Regional assessments extend the findings in the Study Units by filling critical gaps in characterizing the quality of surface water and ground water, and by determining water-quality status and trends at sites that have been consistently monitored for more than a decade. In addition, the regional assessments continue to build an understanding of how natural features and human activities affect water quality. Many of the regional assessments employ modeling and other scientific tools, developed on the basis of data collected at individual sites, to help extend knowledge of water quality to unmonitored, yet comparable areas within the regions. The models thereby enhance the value of our existing data and our understanding of the hydrologic system. In addition, the models are useful in evaluating various resource-management scenarios and in predicting how our actions, such as reducing or managing nonpoint and point sources of contamination, land conversion, and altering flow and (or) pumping regimes, are likely to affect water conditions within a region.

Other activities planned during the second decade include continuing national syntheses of information on pesticides, volatile organic compounds (VOCs), nutrients, trace elements, and aquatic ecology; and continuing national topical studies on the fate of agricultural chemicals, effects of urbanization on stream ecosystems, bioaccumulation of mercury in stream ecosystems, effects of nutrient enrichment on stream ecosystems, and transport of contaminants to public-supply wells. 
The USGS aims to disseminate credible, timely, and relevant science information to address practical and effective water-resource management and strategies that protect and restore water quality. We hope this NAWQA publication will provide you with insights and information to meet your needs, and will foster increased citizen awareness and involvement in the protection and restoration of our Nation's waters.

Matthew C. Larsen

Associate Director for Water 


\section{Contents}

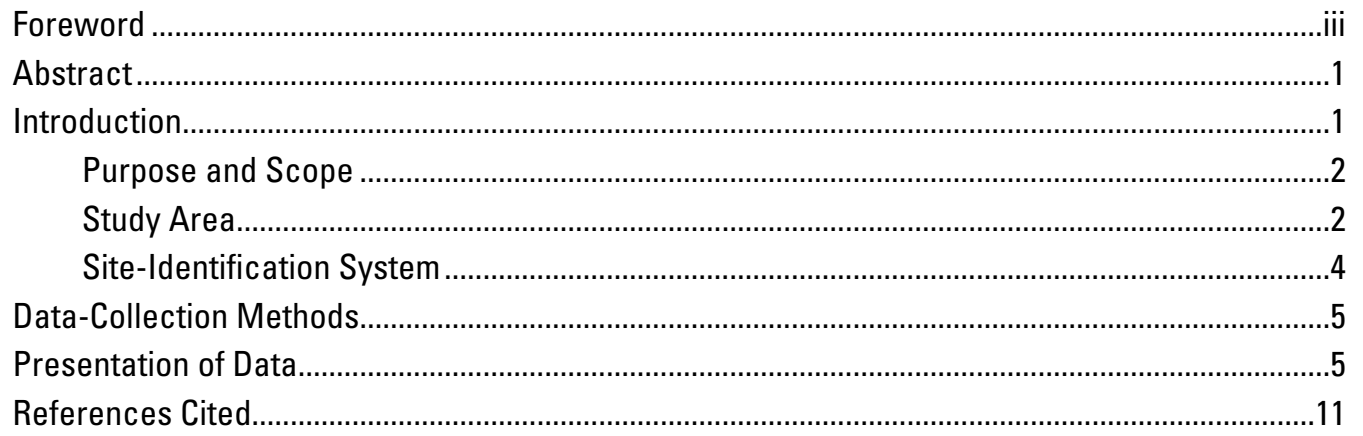

\section{Figures}

1. Maps showing locations of the Bogue Phalia, Tommie Bayou, and unnamed tributary to Clear Creek Basins and piezometers installed along a flowpath along the Bogue Phalia near Leland, MS, in northwestern Mississippi.

2. Hydrograph of streamflow in the Bogue Phalia in Mississippi, 2007-2008, also indicating discharge on dates when surface-water and flowpath samples were collected along the Bogue Phalia near Leland, MS.

3. Schematic diagram of piezometer installation at the flowpath on the Bogue Phalia near Leland, MS

4. Hydrograph of water-surface elevations for the Bogue Phalia near Leland, MS, the alluvial aquifer, and the hyporheic zone, Bogue Phalia Basin, MS, 2006-2008..........6

5-8. Maps showing-

5. Land managers' report of crop types for agricultural fields in the Tommie Bayou Basin, MS, 2007.

6. Land managers' report of crop types for agricultural fields in the Tommie Bayou Basin, MS, 2008.

7. Land managers' report of crop types for agricultural fields in the unnamed tributary to Clear Creek near Napanee, MS, 2007

8. Land managers' report of crop types for agricultural fields in the unnamed tributary to Clear Creek near Napanee, MS, 2008 


\section{Tables}

1. Sites in the Bogue Phalia Basin of Mississippi where water-quality data samples were collected, 2006-2008.

2. Surface-water-quality data for samples collected at station 07288650 ,

Bogue Phalia near Leland, MS, 2006-2008.

3. Groundwater-quality data for samples collected at the flowpath along the Bogue Phalia near Leland, MS, 2006-2008.

4. Surface-water-quality data from samples collected at station 07288636 , Tommie Bayou at Pace, MS.

5. Surface-water-quality data from samples collected at station 333150090530400 , an unnamed tributary to Clear Creek near Napanee, MS

6. Land managers' report of physical and chemical treatments of fields during crop production, Tommie Bayou Basin, Mississippi, 2007.

7. Land managers' report of physical and chemical treatments of fields during crop production, Tommie Bayou Basin, Mississippi, 2008.

8. Land managers' report of physical and chemical treatments of fields during crop production, unnamed tributary to Clear Creek Basin, Mississippi, 2007.

9. Land managers' report of physical and chemical treatments of fields during crop production, unnamed tributary to Clear Creek Basin, Mississippi, 2008. 


\section{Conversion Factors}

\begin{tabular}{|c|c|c|}
\hline Multiply & By & To obtain \\
\hline \multicolumn{3}{|c|}{ Length } \\
\hline inch & 2.54 & centimeter $(\mathrm{cm})$ \\
\hline foot $(\mathrm{ft})$ & 0.3048 & meter $(\mathrm{m})$ \\
\hline mile (mi) & 1.609 & kilometer (km) \\
\hline \multicolumn{3}{|c|}{ Area } \\
\hline acre & 4,047 & square meter $\left(\mathrm{m}^{2}\right)$ \\
\hline acre & 0.4047 & hectare (ha) \\
\hline square mile $\left(\mathrm{mi}^{2}\right)$ & 259.0 & hectare (ha) \\
\hline \multicolumn{3}{|c|}{ Volume } \\
\hline ounce, fluid (fl. oz) & 0.02957 & liter $(\mathrm{L})$ \\
\hline pint (pt) & 0.4732 & liter (L) \\
\hline quart (qt) & 0.9464 & liter (L) \\
\hline gallon (gal) & 3.785 & liter (L) \\
\hline gallon (gal) & 0.003785 & cubic meter $\left(\mathrm{m}^{3}\right)$ \\
\hline \multicolumn{3}{|c|}{ Flow rate } \\
\hline cubic foot per second $\left(\mathrm{ft}^{3} / \mathrm{s}\right)$ & 0.02832 & cubic meter per second $\left(\mathrm{m}^{3} / \mathrm{s}\right)$ \\
\hline
\end{tabular}

Temperature in degrees Celsius $\left({ }^{\circ} \mathrm{C}\right)$ may be converted to degrees Fahrenheit $\left({ }^{\circ} \mathrm{F}\right)$ as follows:

$$
{ }^{\circ} \mathrm{F}=\left(1.8 \times{ }^{\circ} \mathrm{C}\right)+32
$$

Vertical coordinate information is referenced to the North American Vertical Datum of 1988 (NAVD 88) unless otherwise noted.

Horizontal coordinate information is referenced to the North American Datum of 1983 (NAD 83).

Altitude, as used in this report, refers to distance above the vertical datum.

Specific conductance is given in microsiemens per centimeter at 25 degrees Celsius $(\mu \mathrm{S} / \mathrm{cm}$ at $\left.25^{\circ} \mathrm{C}\right)$.

Concentrations of chemical constituents in water are given either in milligrams per liter (mg/L) or micrograms per liter $(\mu \mathrm{g} / \mathrm{L})$. 



\title{
Water-Quality, Water-Level, and Discharge Data Associated with the Mississippi Embayment Agricultural Chemical Transport Study, 2006-2008
}

\author{
By Melinda S. Dalton, Claire E. Rose, and Richard H. Coupe
}

\section{Abstract}

In 2006, the Agricultural Chemicals: Sources, Transport and Fate study team (Agricultural Chemicals Team, ACT) of the U.S. Geological Survey National Water-Quality Assessment Program began a study in northwestern Mississippi to evaluate the influence of surface-water recharge on the occurrence of agriculturally related nutrients and pesticides in the Mississippi River Valley alluvial aquifer. The ACT study was composed in the Bogue Phalia Basin, an indicator watershed within the National Water-Quality Assessment Program Mississippi Embayment Study Unit and utilized several small, subbasins within the Bogue Phalia to evaluate surface and groundwater interaction and chemical transport in the Basin. Data collected as part of this ACT study include water-quality data from routine and incident-driven water samples evaluated for major ions, nutrients, organic carbon, physical properties, and commonly used pesticides in the area; discharge, gage height and water-level data for surface-water sites, the shallow alluvial aquifer, and hyporheic zone; additionally, agricultural data and detailed management activities were reported by land managers for farms within two subbasins of the Bogue Phalia Basin-Tommie Bayou at Pace, MS, and an unnamed tributary to Clear Creek near Napanee, MS.

\section{Introduction}

In 2006, the Mississippi Embayment (MISE) Study Unit of the National Water-Quality Assessment (NAWQA) Program began data-collection activities in the Bogue Phalia Basin in northwestern Mississippi (fig. 1) as part of the U.S. Geological Survey's (USGS) Agricultural Chemicals: Sources, Transport and Fate study (Agricultural Chemicals Team, ACT; Capel and others, 2004). The Bogue Phalia is one of seven watersheds studied nationally to determine the mechanisms and extent to which natural and agricultural factors influence chemical transport and water quality, and it is one of the most agriculturally productive (corn, cotton, rice, and soybeans) areas in the Nation. Data collection and analysis in each ACT study basin is designed similarly, and a multiscale approach is used to evaluate water and chemical transport. An integral part of the multiscale approach is a network of shallow piezometers, in-stream piezometers, and surface-water gages that are used to monitor water levels, streamflow, and water quality.

The ACT study in the Bogue Phalia Basin was designed to (1) describe groundwater and surface-water interactions in the Bogue Phalia Basin; (2) determine if surface water could be contaminating the shallow groundwater system; (3) determine the extent to which applications of pesticides and nutrients for agricultural production affect groundwater and surface-water quality; and (4) apply the information developed as a result of this study to help advance the understanding of processes in similar environmental settings.

The data-collection network for Bogue Phalia consisted of a series of shallow groundwater and in-stream piezometers installed along a flowpath crossing the Bogue Phalia near Leland, Mississippi (fig. 1). Continuous and synoptic water levels (available at the National Water Information System Web site, http://waterdata.usgs.gov/nwis) were recorded in each piezometer during May 2006-November 2008. A USGS real-time surface-water monitoring station in operation less than a mile downstream from the flowpath provided continuous measurements of gage height, discharge, and precipitation. Surface-water and groundwater samples were collected quarterly beginning in 2006 and during major storms occurring in June and July 2007 and February 2008. Samples were analyzed for physical properties, major ions, sulfide, organic carbon, nutrients, and pesticides. Additionally, routine surface-water-quality samples were collected from the Bogue Phalia weekly or biweekly as part of the NAWQA assessment of the status and trends of the Nation's major river basins (U.S. Geological Survey, 2006). In two subbasins-Tommie Bayou at Pace, MS, and unnamed tributary to Clear Creek near Napanee, MS - automatic samplers were installed to collect stormflow samples. These samples were analyzed for physical properties, major ions, sulfide, nutrients, and pesticides. 


\section{Purpose and Scope}

This report presents water-quality, water-level, stage, and discharge data for both groundwater and surface-water sites in the Bogue Phalia Basin in northwestern Mississippi during 2006-2008, and reported agricultural land-management activities for two small subbasins from 2007 to 2008. The data compiled in this report include field and laboratory chemical analyses of both shallow groundwater and surface-water samples collected in the Bogue Phalia Basin. In addition, the report includes reported agricultural-management activities in two subbasins in the Bogue Phalia Basin, and water-level data from shallow piezometers in the alluvial aquifer. Groundwater-quality data were collected quarterly in 13 piezometers (shallow and instream), and water-level data were collected continuously from 9 piezometers and quarterly from all 13 during water-quality sampling visits (tables 1-3 [tables 2 and 3, back of report]). Surface-water-quality data, stage, and discharge were collected as part of routine NAWQA sampling at the Bogue Phalia near Leland, MS, and during stormflow events at Tommie Bayou at Pace, MS, and an unnamed tributary to Clear Creek near Napanee, MS.

Data presented in this report are being used to develop groundwater-flow models, evaluate groundwater and surfacewater interactions in similar agricultural settings, and determine the potential for the hydrology of the Bogue Phalia Basin to transmit agricultural chemicals. Additionally, data presented in this report will be used in a regional evaluation of the effects of hydrology, geology, and land-management activities on water quality and chemical transport not only in the MISE study area but in similar study areas across the Nation as part of the NAWQA ACT program.

\section{Study Area}

The Mississippi Delta, a 7,000-square-mile area in northwestern Mississippi (fig. 1), is one of the most agriculturally fertile areas in the United States. Composed of flood-plain soils, the Mississippi Delta is underlain by the Mississippi River Valley alluvial aquifer, the major source of water in the region and the most heavily used aquifer in the State. Rainfall averages 52 inches per year; however, agricultural practices in the area often require irrigation. Water from the alluvial aquifer is used mostly for agricultural and industrial
Table 1. Sites in the Bogue Phalia Basin of Mississippi where water-quality data samples were collected, 2006-2008.

\begin{tabular}{llc}
$\begin{array}{l}\text { [Site type: SW_-surface water, HW_hyporheic zone, GW—-groundwater; } \\
\text { see fig. 1 for locations] }\end{array}$ & \multicolumn{1}{c}{ Station name } & Site type \\
\hline Station identifier & \multicolumn{1}{c}{ Tommie Bayou at Pace, MS } & SW \\
\hline 07288636 & Bogue Phalia near Leland, MS & SW \\
07288650 & Right bank piezometer (RB) & HW \\
332440090502001 & Right channel piezometer (RC) & HW \\
332440090502195 & Center channel piezometer (C) & HW \\
332440090502196 & Left channel piezometer (LC) & HW \\
332440090502197 & Left bank piezomter (LB) & HW \\
332440090502201 & Flow system well 1 (FS-1A) & GW \\
332443090502301 & Flow system well 1 (FS-1B) & GW \\
332443090502302 & Flow system well 2 (FS-2A) & GW \\
332443090502501 & Flow system well 2 (FS-2B) & GW \\
332443090502502 & Flow system well 3 (FS-3A) & GW \\
332443090502701 & Flow system well 3 (FS-3B) & GW \\
332443090502702 & Aerial recharge well 1 (AR-1A) & GW \\
332445090501601 & Aerial recharge well 1 (AR-1B) & GW \\
332445090501602 & Unnamed tributary to Clear & SW \\
333150090530400 & Creek near Napanee, MS & \\
& &
\end{tabular}

purposes, and is not used for drinking. Land use is dominated by agriculture (corn, cotton, soybeans, and rice); as a result, agricultural chemicals are used heavily and have been detected in surface water and rainfall since the 1990s (Coupe, 2000, 2002; Coupe and Capel, 2005).

The hydrology of the alluvial aquifer has been studied previously by Arthur (2001), Boswell and others (1968), and Snider and Sanford (1981). Multiple streams and rivers incise the alluvial aquifer in the study area, contributing to streamflow in the Bogue Phalia from the headwaters near Gunnison, MS, to where it converges with the Big Sunflower River near Darlove, MS. Seasonal fluctuations in streamflow (fig. 2) are dominated by agricultural pumping and agricultural return flow The estimated annual recharge to the aquifer from precipitation is 2.6 inches (Arthur, 2001). 

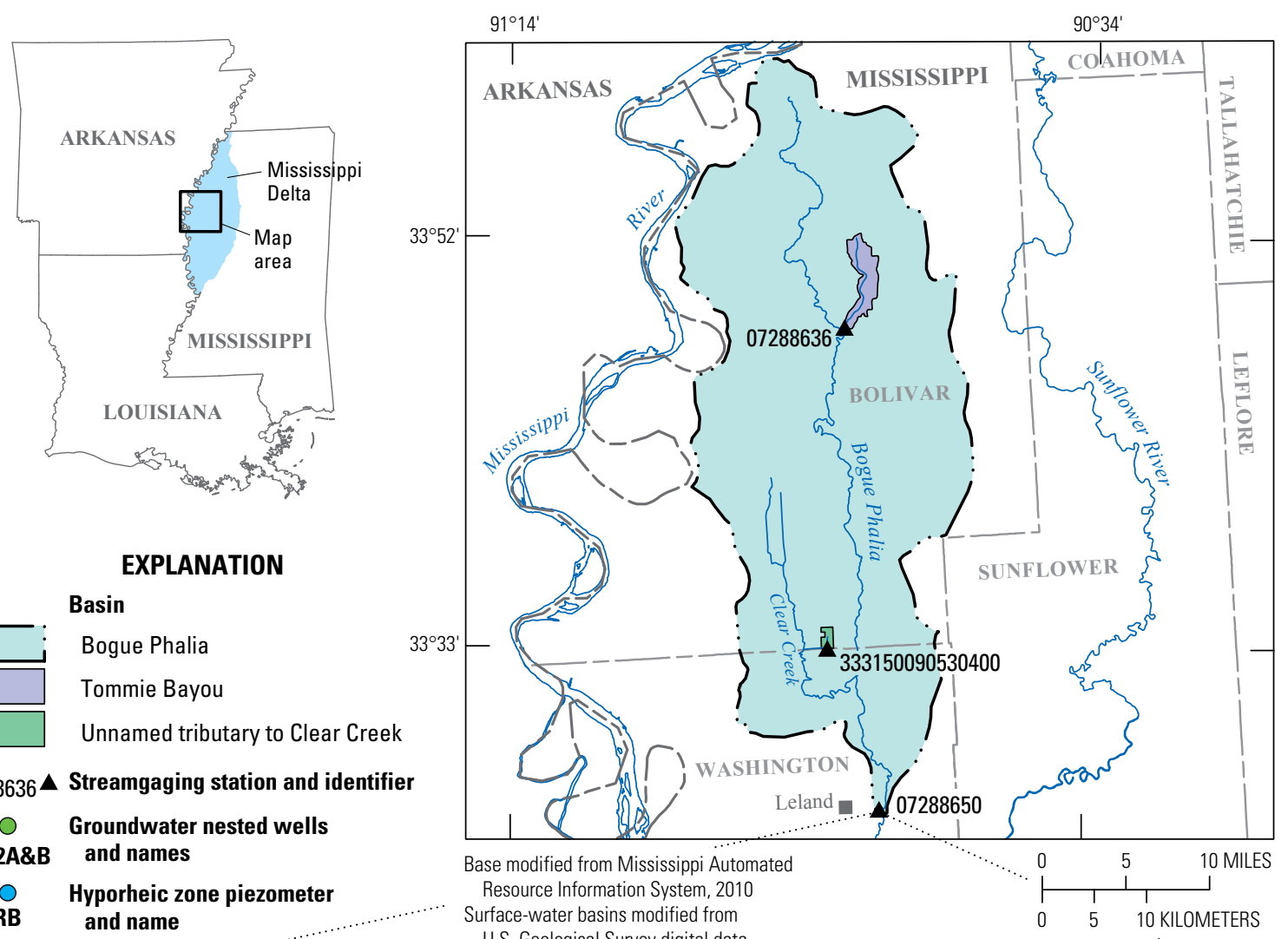

Basin

Bogue Phalia

Tommie Bayou

Unnamed tributary to Clear Creek

$07288636 \Delta$ Streamgaging station and identifier

Groundwater nested wells

FS-2A\&B and names

RB Hyporheic zone piezometer

and name

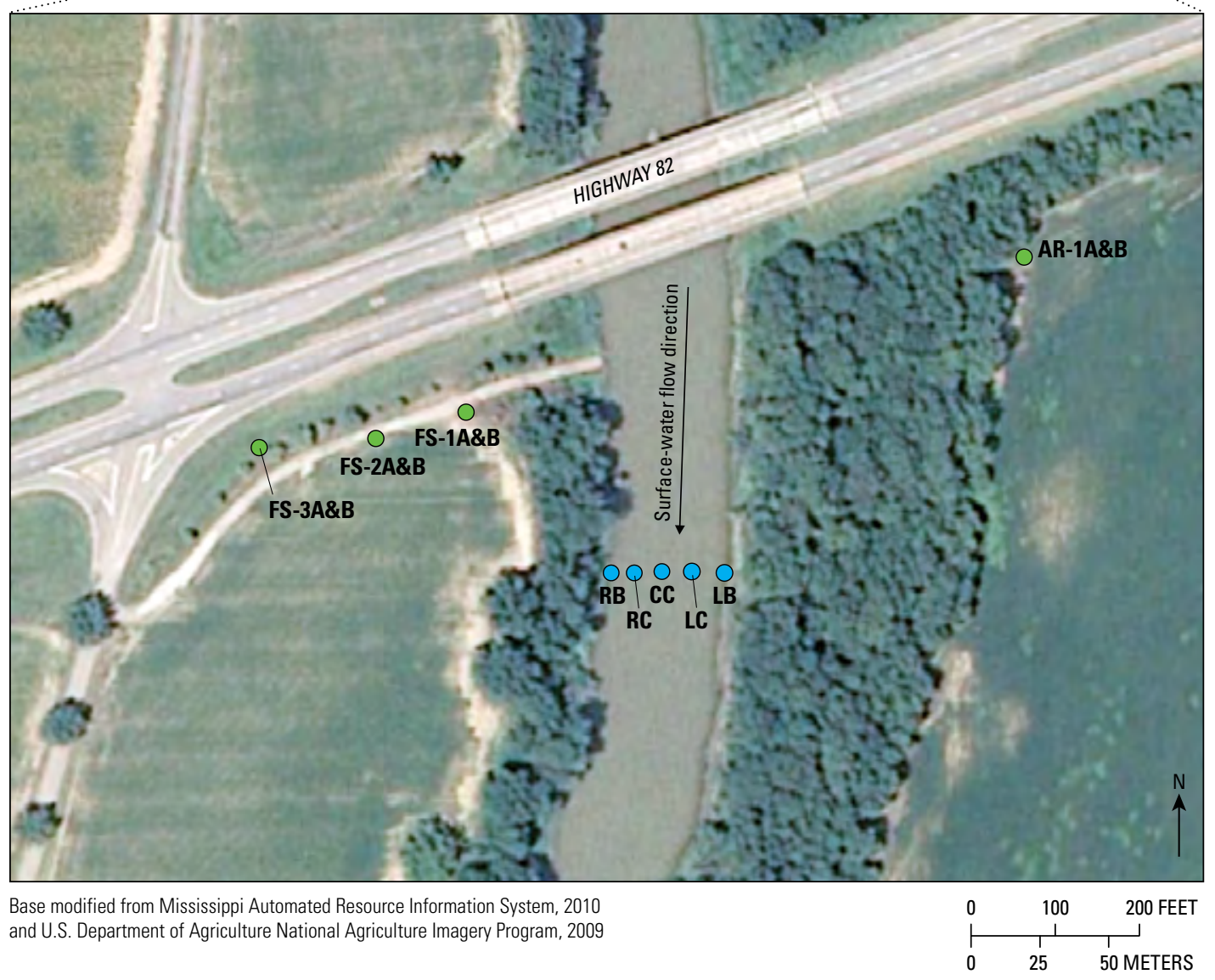

Figure 1. Locations of $(A)$ the Bogue Phalia, Tommie Bayou, and unnamed tributary to Clear Creek Basins and $(B)$ piezometers installed along a flowpath along the Bogue Phalia near Leland, MS, in northwestern Mississippi (see table 1). 


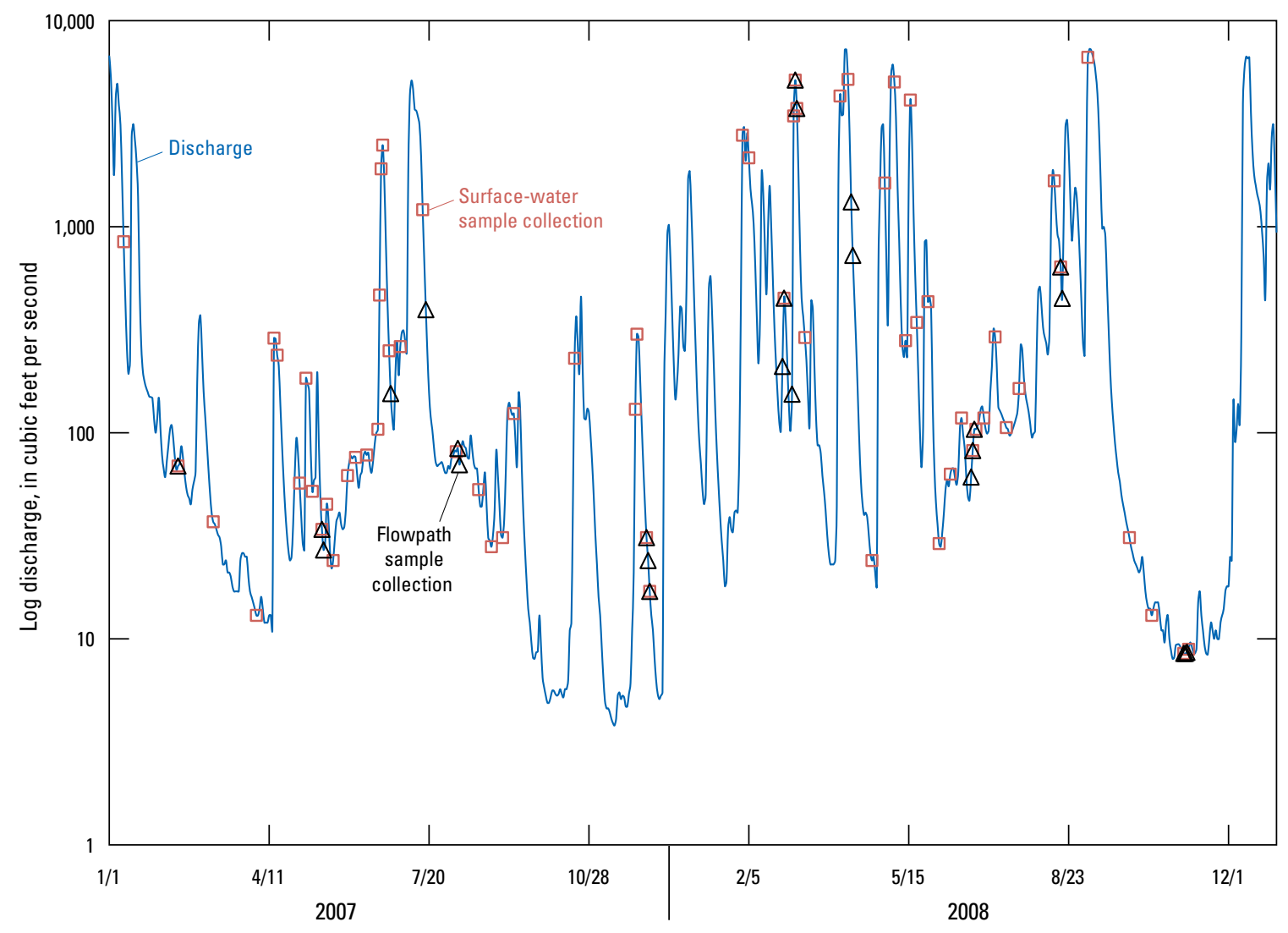

Figure 2. Hydrograph of streamflow in the Bogue Phalia in Mississippi, 2007-2008, also indicating discharge on dates when surface-water and flowpath samples were collected along the Bogue Phalia near Leland, MS.

\section{Site-Identification System}

In this report, piezometers are identified two ways-by using the 15-digit station identification (ID) numbers and field ID numbers. The 15-digit station ID number is constructed by using the latitude and longitude of the well location. Piezometers inventoried at the same location, to the minute, are numbered sequentially beginning with " 01 ." For example, the station ID number for the second piezometer in a cluster of piezometers would be 312715085364202 , with the last two digits identifying it as the second piezometer in the cluster. Field IDs are abbreviations of piezometer type; for example, FS for flow-system piezometers and AR for areal recharge piezometers. The ID for in-stream piezometers indicates the piezometer location in the stream channel; for instance, the ID for the piezometer closest to the right bank is RB. The FS piezometers were developed in both shallow and deeper zones of the alluvial aquifer and were further defined by using an " $\mathrm{A}$ " to designate the deeper piezometers and "B" to designate the shallower piezometers (fig. 3 ).
Streams are identified by downstream-order numbers according to the guidelines presented in USGS Annual Data Reports (U.S. Geological Survey, 2010).

"Since October 1, 1950, hydrologic-station records in USGS reports have been listed in order of downstream direction along the main stream. All stations on a tributary entering upstream from a main-stream station are listed before that station. A station on a tributary entering between two mainstream stations is listed between those stations. A similar order is followed in listing stations on first rank, second rank, and other ranks of tributaries. The rank of any tributary on which a station is located with respect to the stream to which it is immediately tributary to is indicated by an indention in that list of stations in the front of this report. Each indentation represents one rank. This downstream order and system of indentation indicates which stations are on tributaries between any two stations and the rank of the tributary on which each station is located. 




\section{EXPLANATION}

Figure 3. Schematic of piezometer installation at the flowpath on the Bogue Phalia near Leland, MS.



As an added means of identification, each hydrologic station and partial-record station has been assigned a station number. These station numbers are in the same downstream order used in this report. In assigning a station number, no distinction is made between partial-record stations and other stations; therefore, the station number for a partial-record station indicates downstream-order position in a list composed of both types of stations. Gaps are consecutive. The complete 8-digit (or 10-digit) number for each station such as 09004100 , which appears just to the left of the station name, includes a 2-digit part number " 09 " plus the 6-digit (or 8-digit) downstream order number "004100." In areas of high station density, an additional two digits may be added to the station identification number to yield a 10-digit number. The stations are numbered in downstream order as described above between stations of consecutive 8-digit numbers."

\section{Data-Collection Methods}

Water-level data were collected both continuously with pressure transducers and during site visits and sampling events. Continuous data were collected with transducers deployed in individual piezometers. Data were downloaded periodically in the field, taken back to the office and processed, and a final value of depth to water from land surface was recorded and stored in the USGS National Water Information System (NWIS, http://waterdata.usgs.gov/nwis). Water levels in piezometers also were recorded during water-quality sampling as part of the USGS groundwater sampling protocol (U.S. Geological Survey, variously dated). Surface-water discharge and stage measurements were collected according to the procedures outlined in Rantz and others, 1982.

Water-quality data were collected for both surface and groundwater samples following standard sampling protocols described by Koterba and others (1995) and the USGS National Field Manual (U.S. Geological Survey, variously dated). Field measurements of stage, discharge, and depth to water level, water temperature, specific conductance, dissolved oxygen, $\mathrm{pH}$, and turbidity were collected at each site. Water samples were collected and sent to multiple laboratories for analysis. When data were available, they were processed and checked at the office and made available on NWIS.

\section{Presentation of Data}

Water-quality data collected during this study are listed in tables 2-5 (back of report) and organized by site and date. Fluctuations in water levels in the alluvial aquifer and hyporheic zone and surface-water discharge are shown in figure 4; a subset of these data also can be found in tables 2 and 3 . Land managers reported physical and chemical treatments of agricultural land in the basins of Tommie Bayou (figs. 5, 6) and unnamed tributary to Clear Creek (figs. 7, 8) for 2007 and 2008 are described in tables 6-9 (back of report). 


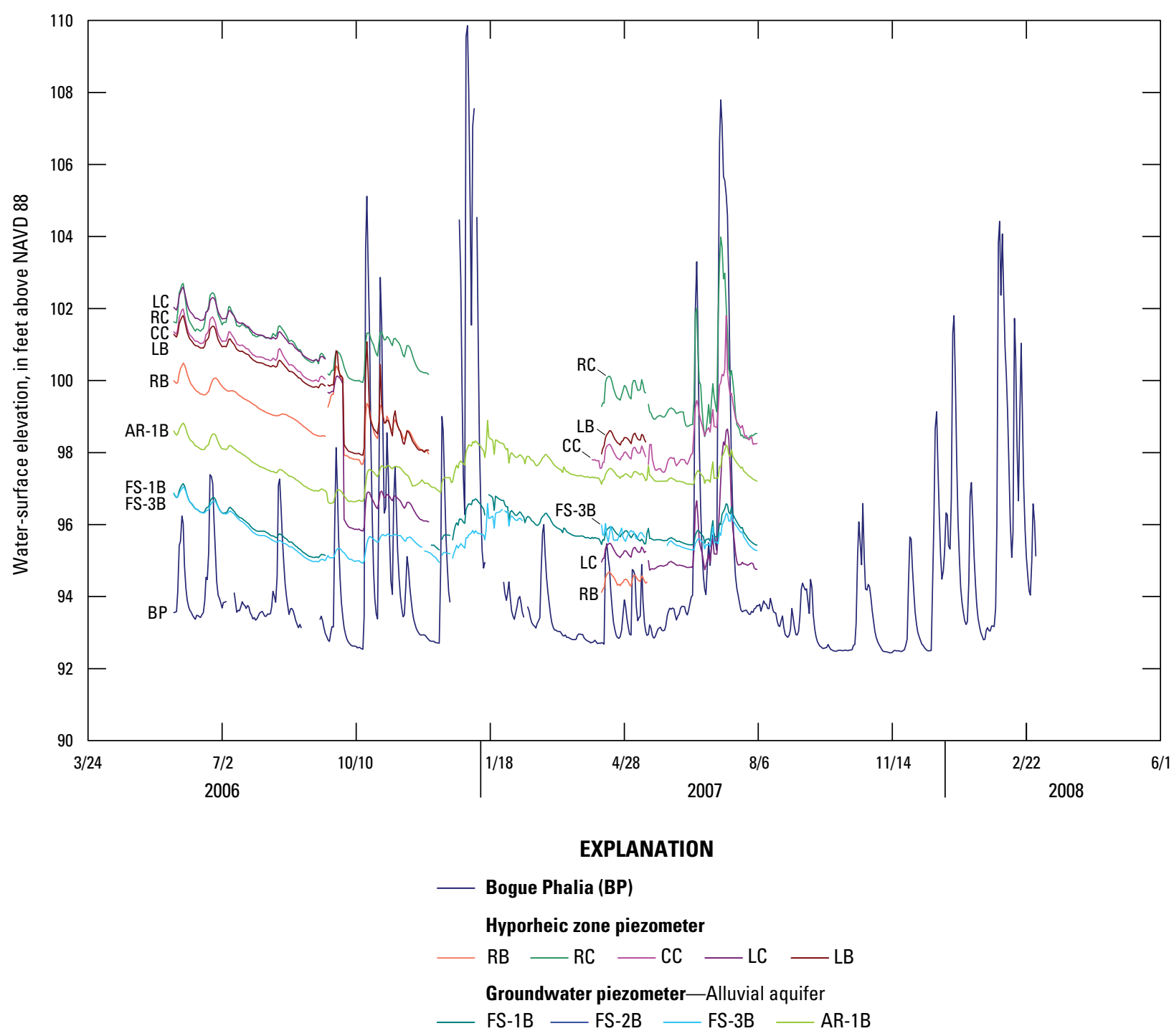

Figure 4. Hydrograph of water-surface elevations for the Bogue Phalia near Leland, MS, the alluvial aquifer, and the hyporheic zone, Bogue Phalia Basin, MS, 2006-2008. See figure 1 for locations. 




Figure 5. Land managers' report of crop types for agricultural fields in the Tommie Bayou Basin, MS, 2007. 


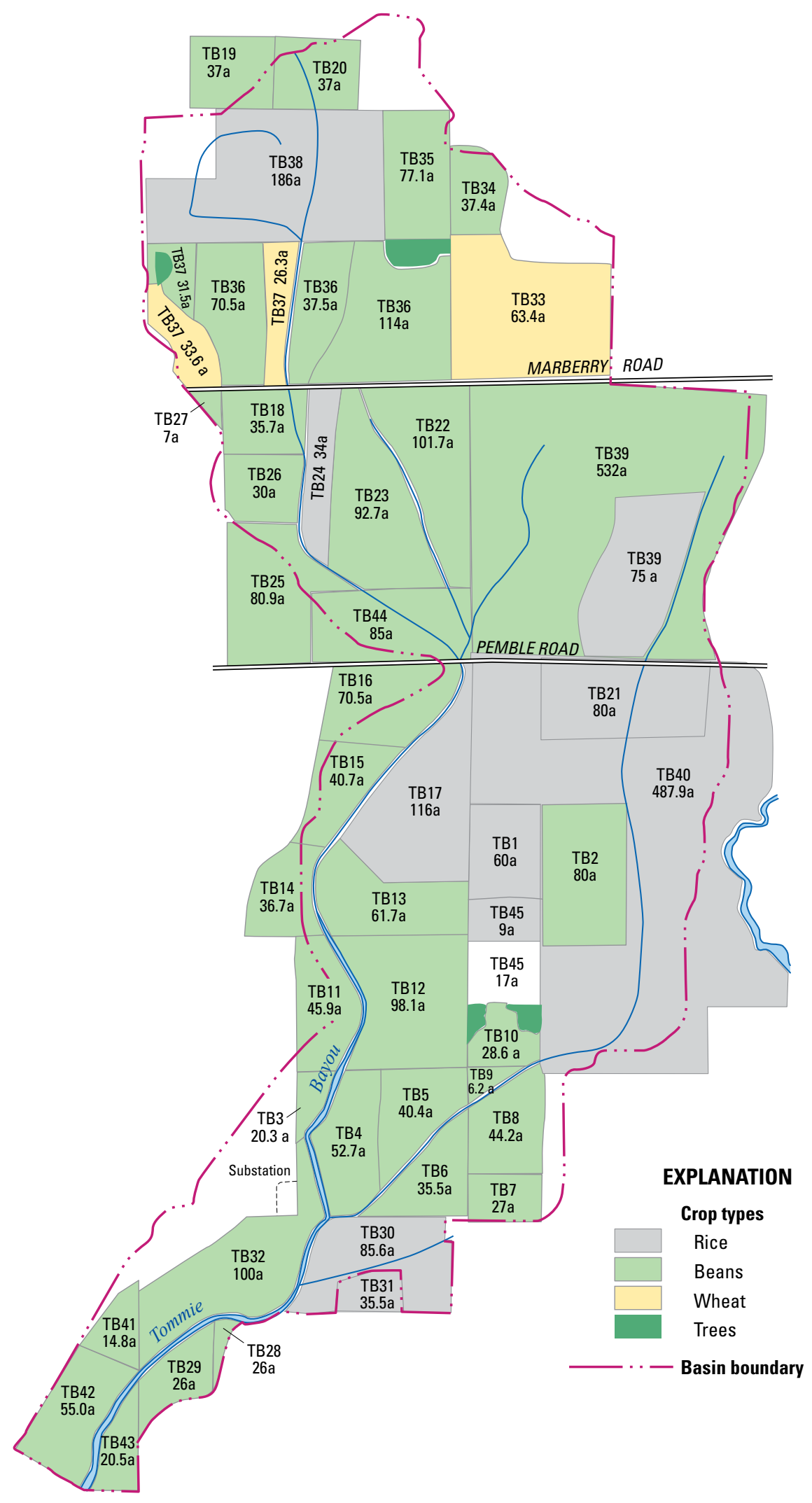

Figure 6. Land managers' report of crop types for agricultural fields in the Tommie Bayou Basin, MS, 2008. 


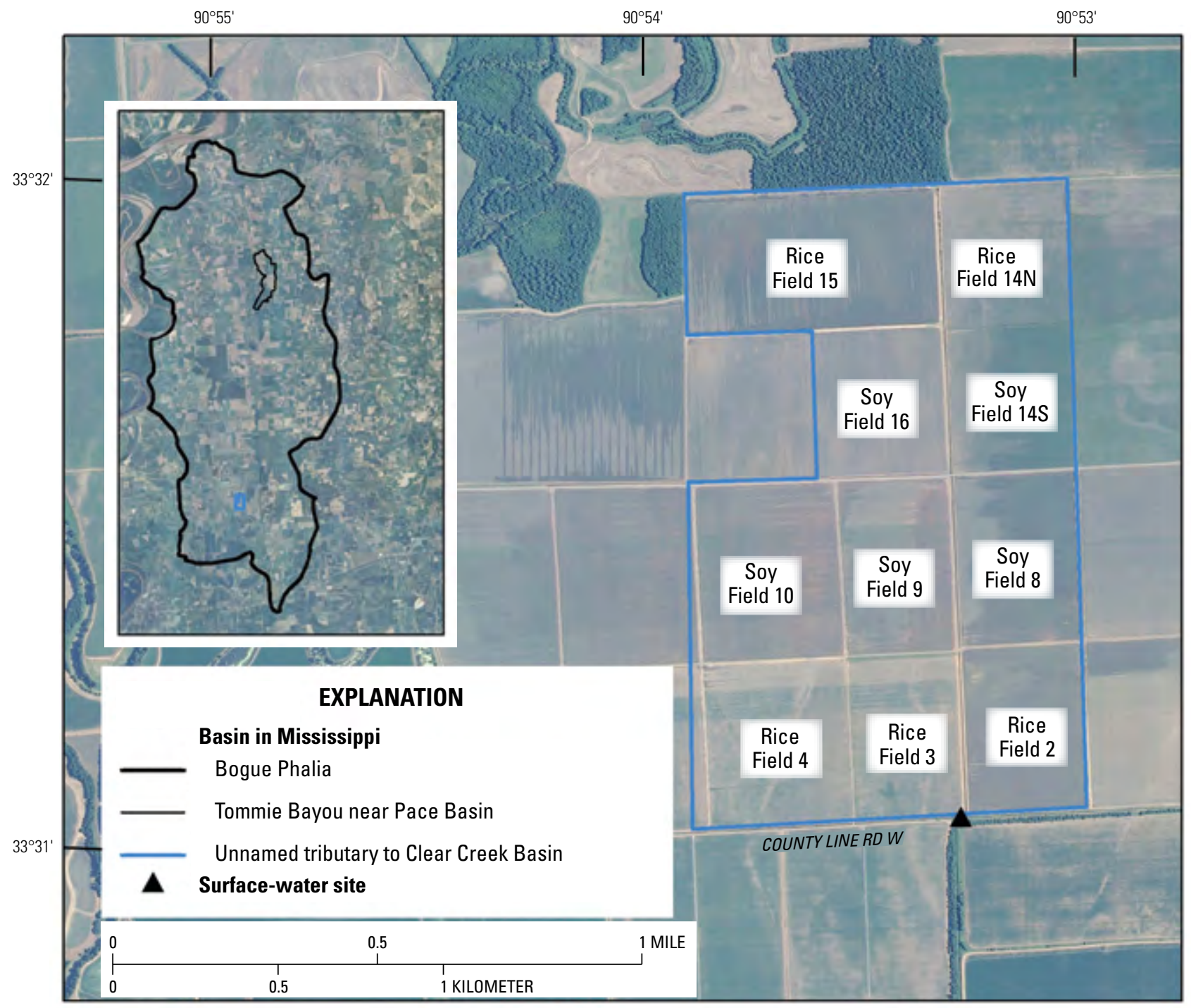

Base modified from Mississippi Automated Resource Information System, 2010 and U.S. Department of Agriculture National Agriculture Imagery Program, 2009

Figure 7. Land managers' report of crop types for agricultural fields in the unnamed tributary to Clear Creek near Napanee, MS, 2007. 


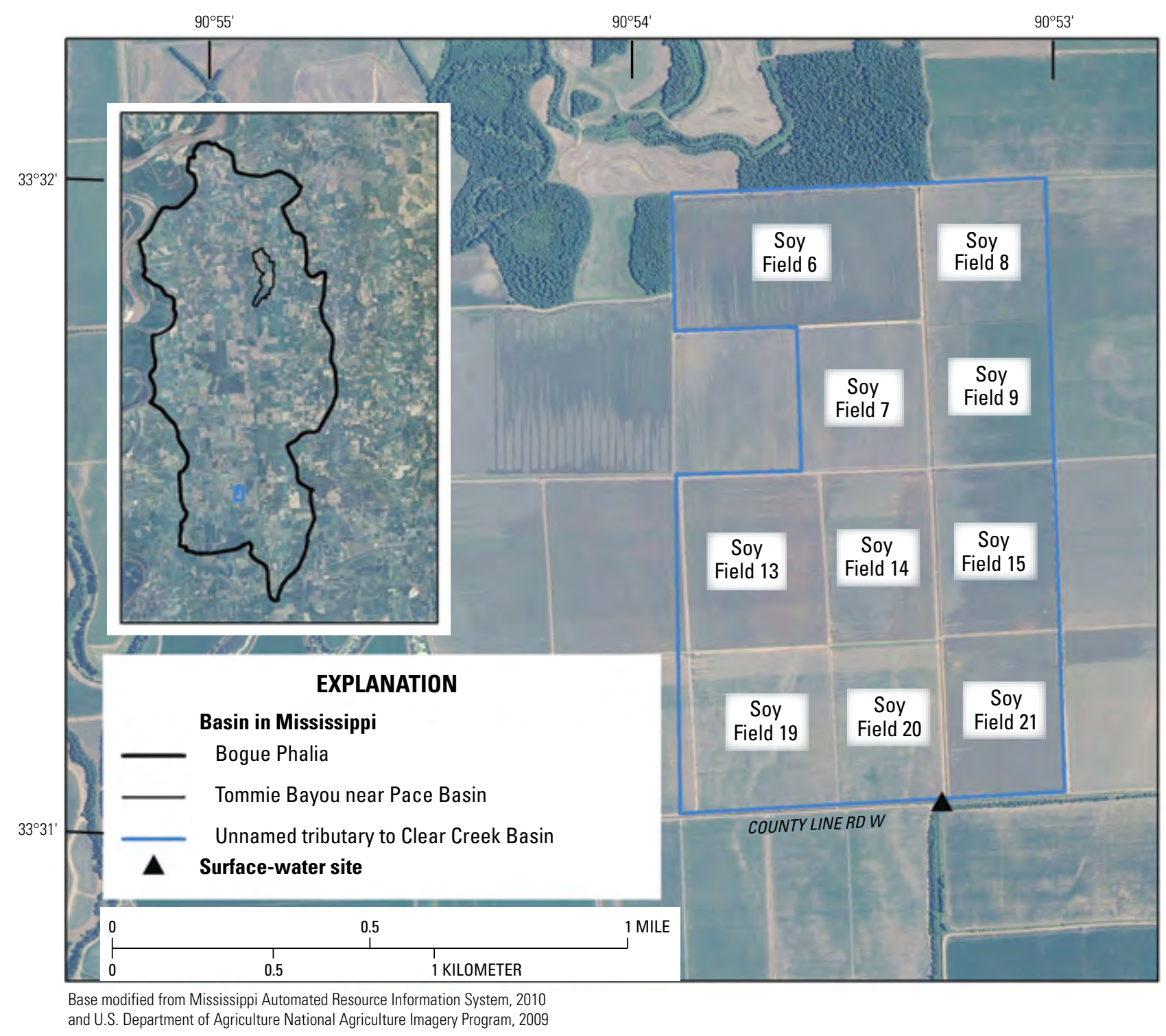

Figure 8. Land managers' report of crop types for agricultural fields in the unnamed tributary to Clear Creek near Napanee, MS, 2008. 


\section{References Cited}

Arthur, J.K., 2001, Hydrogeology, model description, and flow analysis of the Mississippi River alluvial aquifer in northwestern Mississippi: U.S. Geological Survey WaterResources Investigations Report 01-4035, p. 40-45.

Boswell, E.H., Cushing, E.M., and Hosman, R.L., 1968, Quaternary aquifer in the Mississippi Embayment, with a discussion of quality of water by Jeffery, H.G.: U.S. Geological Survey Professional Paper 448-E, 15 p.

Capel, P.D., Hamilton, P.A., and Erwin, M.L., 2004, Studies by the U.S. Geological Survey on sources, transport, and fate of agricultural chemicals: U.S. Geological Survey Fact Sheet 2004-3098, 4 p.

Coupe, R.H., 2000. Occurrence of pesticides in five rivers of the Mississippi Embayment Study Unit, 1996-98: U.S. Geological Survey Water-Resources Investigations Report 99-4159, 30 p.

Coupe, R.H., 2002, Nitrogen and phosphorus concentrations and fluxes of streams in the Mississippi Embayment Study Unit, 1996-98: U.S. Geological Survey Water-Resources Investigations Report 01-4024, p. 3-5.

Coupe, R.H., and Capel, P.D., 2005, Bogue Phalia watershed in Mississippi selected for a national water-quality study: U.S. Geological Survey Fact Sheet 2005-3050, 2 p.
Koterba, M.T., Wilde, F.D., and Lapham, W.W., 1995, Ground-water data-collection protocols and procedures for the National Water-Quality Assessment ProgramCollection and documentation of water-quality samples and related data: U.S. Geological Survey Open-File Report 95-399, 113 p.

Rantz, S.E., and others, 1982, Measurement and computation of streamflow_-Volume 1. Measurement of stage and discharge: U.S. Geological Survey Water-Supply Paper 2175, 284 p.

Snider, J.L., and Sanford, T.H., Jr., 1981, Water resources of the Terrace aquifers, central Louisiana: Louisiana Department of Transportation and Development, Water Resources Technical Report No. 25, 48 p.

U.S. Geological Survey, 2006, About regional assessments on water-quality status and trends: U.S. Geological Survey National Water-Quality Assessment (NAWQA) Program, accessed May 15, 2010, at http://water.usgs.gov/nawqa/ studies/regional_assessments.html.

U.S. Geological Survey, 2010, Introductory material: Details and explanations regarding site numbering, data reported, and accuracy of data for Annual Data Reports, at http://wdr. water.usgs.gov/wy2010/documentation.html\#sitenumber.

U.S. Geological Survey, variously dated, National field manual for the collection of water-quality data: U.S. Geological Survey Techniques of Water-Resources Investigations, book 9, chaps. A1-A9. 



\section{Tables 2 through 9}




\section{Data Associated with the Mississippi Embayment Agricultural Chemical Transport Study, 2006-2008}

Table 2. Surface-water-quality data for samples collected at station 07288650, Bogue Phalia near Leland, MS, 2006-2008.

[NGVD 29, National Geodetic Vertical Datum of 1929; mg/L, milligram per liter; $\mu \mathrm{g} / \mathrm{L}$, microgram per liter, -, no data; E, estimated; see fig. 1 for location]

\begin{tabular}{|c|c|c|c|c|c|c|c|c|}
\hline $\begin{array}{l}\text { Sample } \\
\text { date }\end{array}$ & $\begin{array}{l}\text { Water-surface } \\
\text { elevation } \\
\text { (feet above } \\
\text { NGVD 29) }\end{array}$ & $\begin{array}{l}\text { Gage } \\
\text { height } \\
\text { (fieet) }\end{array}$ & $\begin{array}{l}\text { Discharge } \\
\text { (subic feet } \\
\text { per second) }\end{array}$ & $\begin{array}{l}\text { Turbidity, in } \\
\text { Nephelometric } \\
\text { Turbiditity Units }\end{array}$ & $\begin{array}{c}\text { Dissolved } \\
\text { oxygen } \\
\text { (mg/L) }\end{array}$ & pH & $\begin{array}{c}\text { Specific } \\
\text { conductance, } \\
\text { (microsiemens } \\
\text { per centimeterl }\end{array}$ & $\begin{array}{c}\text { Water } \\
\text { temperatur } \\
\text { (degres } \\
\text { Celsisus) }\end{array}$ \\
\hline
\end{tabular}

\begin{tabular}{lrrrrrrrr}
\hline $1 / 9 / 2006$ & 92.79 & 6.58 & 14 & 24 & 14.3 & 7.7 & 428 & 12.9 \\
$3 / 6 / 2006$ & 93.82 & 7.61 & 80 & 190 & 8.7 & 7.1 & 191 & 14.9
\end{tabular}

$1 / 9 / 2006$

$3 / 6 / 2006$

$5 / 2 / 2006$

$7 / 18 / 2006$

$8 / 1 / 2006$

$8 / 1 / 2006$

9/12/2006

$11 / 15 / 2006$

$12 / 4 / 2006$

$\begin{array}{ll}6.58 & 14 \\ 7.61 & 80\end{array}$

93.54

7.33

93.82
93.78 $\quad 7.61$

$\begin{array}{ll}93.78 & 7.57 \\ 93.57 & 7.36\end{array}$

$\begin{array}{ll}93.57 & 7.36\end{array}$

$\begin{array}{ll}93.89 & 6.68 \\ 93.91 & 7.70\end{array}$

1/10/2007

2/13/200

3/7/2007

4/14/2007

4/16/2007

4/30/2007

$5 / 8 / 2007$

$5 / 14 / 2007$

$5 / 17 / 2007$

$5 / 21 / 2007$

6/4/2007

6/11/2007

6/18/2007

6/19/2007

6/20/2007

$6 / 21 / 2007$

7/2/2007

$7 / 16 / 2007$

7/30/2007

$8 / 6 / 2007$

$9 / 4 / 2007$

9/11/2007

14
80
58
2,920
87
88
73
73
21
96
57
17
767
$\mathrm{E} 69$
36
12
461
235
56
241
49
33
47
25
62
76
80
103
832
2,120
2,560
234
323
1,110
64
80
51
35
30
122

Table 2. Surface-water-quality data for samples collected at station 07288650, Bogue Phalia near Leland, MS, 2006-2008. - Continued

[NGVD 29, National Geodetic Vertical Datum of 1929; $\mathrm{mg} / \mathrm{L}$, milligram per liter; $\mu \mathrm{g} / \mathrm{L}$, microgram per liter; —, no data; $\mathrm{E}$, estimated; see fig. 1 for location]

\begin{tabular}{|c|c|c|c|c|c|c|c|c|}
\hline $\begin{array}{c}\text { Sample } \\
\text { date }\end{array}$ & $\begin{array}{l}\text { Calcium } \\
\text { (mg/L) }\end{array}$ & $\begin{array}{c}\text { Magnesium } \\
(\mathrm{mgl/L})\end{array}$ & $\begin{array}{c}\text { Potassium } \\
(\mathrm{mg} / \mathrm{L})\end{array}$ & $\begin{array}{c}\text { Sodium } \\
\text { (my/L) }\end{array}$ & $\begin{array}{l}\text { Alkalinity, } \\
\text { field, } \\
\text { (myl/ as } \\
\text { calcium } \\
\text { carbonate) }\end{array}$ & $\begin{array}{c}\text { Bicarbonate, } \\
\text { field } \\
\text { (mgl/) }\end{array}$ & $\begin{array}{c}\text { Chloride } \\
\text { (mg/L) }\end{array}$ & $\begin{array}{c}\text { Fluoride } \\
\text { (mg/L) }\end{array}$ \\
\hline 1/9/2006 & - & - & - & - & 169 & 205 & 6.39 & - \\
\hline 3/6/2006 & - & - & - & - & 72 & 87 & 3.48 & - \\
\hline $4 / 3 / 2006$ & - & - & - & - & 88 & 108 & 3.29 & - \\
\hline $5 / 2 / 2006$ & - & - & - & - & 19 & 23 & 1.60 & - \\
\hline 7/12/2006 & - & - & - & - & 194 & 231 & 8.98 & - \\
\hline 7/18/2006 & - & - & - & - & - & - & - & - \\
\hline $8 / 1 / 2006$ & - & - & - & - & - & - & - & - \\
\hline $8 / 1 / 2006$ & - & - & - & - & - & - & - & - \\
\hline 9/12/2006 & - & - & - & - & 241 & 289 & 12.0 & - \\
\hline $10 / 24 / 2006$ & 27.6 & 8.31 & 6.58 & 7.93 & 88 & 107 & 5.93 & 0.17 \\
\hline $11 / 15 / 2006$ & 26.2 & 7.57 & 5.18 & 7.63 & 88 & 107 & 6.10 & 0.16 \\
\hline $12 / 4 / 2006$ & 48.9 & 14.8 & 4.66 & 13.9 & 158 & 192 & 9.75 & 0.17 \\
\hline $1 / 10 / 2007$ & 11.7 & 3.63 & 3.02 & 3.90 & 43 & 53 & 1.93 & 0.11 \\
\hline 2/13/2007 & 20.7 & 6.03 & 2.87 & 5.92 & 72 & 88 & 3.95 & 0.12 \\
\hline 3/7/2007 & 29.5 & 8.55 & 3.36 & 7.83 & 99 & 120 & 5.25 & 0.16 \\
\hline 4/3/2007 & 60.4 & 18.6 & 4.44 & 11.8 & 179 & 216 & 6.03 & 0.18 \\
\hline 4/14/2007 & 38.2 & 11.3 & 3.17 & 9.06 & 121 & 145 & 5.70 & 0.17 \\
\hline 4/16/2007 & 19.5 & 5.57 & 4.00 & 7.46 & 53 & 64 & 5.45 & 0.15 \\
\hline $4 / 30 / 2007$ & 26.1 & 8.08 & 4.59 & 11.3 & 85 & 103 & 7.26 & 0.19 \\
\hline $5 / 4 / 2007$ & 31.0 & 10.3 & 4.28 & 11.4 & 96 & E116 & 7.05 & 0.18 \\
\hline $5 / 8 / 2007$ & 22.0 & 6.67 & 4.18 & 10.4 & 76 & 93 & 6.35 & 0.16 \\
\hline $5 / 14 / 2007$ & 25.3 & 8.06 & 4.89 & 9.16 & 81 & 99 & 5.45 & 0.21 \\
\hline $5 / 17 / 2007$ & - & - & - & - & - & - & - & - \\
\hline $5 / 21 / 2007$ & 38.0 & 12.0 & 3.89 & 10.9 & 124 & 149 & 5.24 & 0.23 \\
\hline $5 / 30 / 2007$ & 55.5 & 21.8 & 4.64 & 23.8 & 170 & 204 & 9.35 & 0.26 \\
\hline 6/4/2007 & 61.1 & 22.2 & 4.78 & 23.2 & 189 & 226 & 10.5 & 0.28 \\
\hline 6/11/2007 & 66.3 & 22.6 & 4.30 & 29.8 & 198 & 236 & 13.0 & 0.27 \\
\hline 6/18/2007 & 71.9 & 26.0 & 4.04 & 26.6 & 245 & 242 & 10.0 & 0.32 \\
\hline 6/19/2007 & 25.3 & 8.12 & 3.57 & 8.53 & 75 & 91 & 3.78 & 0.20 \\
\hline 6/20/2007 & 26.4 & 8.05 & 3.83 & 11.0 & 88 & 107 & 5.19 & 0.20 \\
\hline $6 / 21 / 2007$ & 32.8 & 10.9 & 4.01 & 12.0 & 108 & 131 & 5.19 & 0.23 \\
\hline $6 / 25 / 2007$ & 36.7 & 13.0 & 3.81 & 16.1 & 139 & 168 & 5.16 & 0.26 \\
\hline $7 / 2 / 2007$ & 21.3 & 7.48 & 3.46 & 7.26 & 46 & 55 & 3.11 & 0.15 \\
\hline $7 / 16 / 2007$ & 21.0 & 6.14 & 3.79 & 5.76 & 79 & 98 & 2.60 & 0.15 \\
\hline $7 / 30 / 2007$ & 51.9 & 17.8 & 2.84 & 21.1 & 184 & 222 & 8.15 & 0.23 \\
\hline $8 / 6 / 2007$ & 61.1 & 21.5 & 4.29 & 25.2 & 268 & 321 & 11.0 & 0.24 \\
\hline $8 / 20 / 2007$ & 68.6 & 23.9 & 4.07 & 35.3 & 292 & 348 & 14.5 & 0.28 \\
\hline $8 / 28 / 2007$ & 76.7 & 28.2 & 4.49 & 30.0 & 250 & 298 & 12.4 & 0.24 \\
\hline 9/4/2007 & 60.3 & 22.0 & 5.90 & 24.2 & 214 & 256 & 9.00 & 0.24 \\
\hline 9/11/2007 & 44.7 & 18.1 & 5.54 & 16.8 & 232 & 280 & 8.16 & 0.24 \\
\hline
\end{tabular}


Table 2. Surface-water-quality data for samples collected at station 07288650, Bogue Phalia near Leland, MS, 2006-2008. - Continued

[NGVD 29, National Geodetic Vertical Datum of 1929; mg/L, milligram per liter, mg/L, microgram per liter, -, no data; E, estimated; see fig. 1 for location]

\begin{tabular}{|c|c|c|c|c|c|c|c|c|c|}
\hline $\begin{array}{c}\text { Sample } \\
\text { date }\end{array}$ & $\begin{array}{c}\text { Silica } \\
\text { (mm/ as } \\
\text { silica } \\
\text { dioxide) }\end{array}$ & $\begin{array}{l}\text { Sulfate } \\
\text { (mg/L/L) }\end{array}$ & $\begin{array}{l}\text { Ammonia, } \\
\text { fitereded } \\
\text { (mygl/as } \\
\text { nitrogen) }\end{array}$ & $\begin{array}{c}\text { Nitrate } \\
\text { plus nitrite, } \\
\text { filtered, } \\
\text { (my/Las } \\
\text { nitrogen) }\end{array}$ & $\begin{array}{l}\text { Nitrite, } \\
\text { fittered } \\
\text { (my/l/a as } \\
\text { nitrogen) }\end{array}$ & $\begin{array}{c}\text { Particulate } \\
\text { nitrogen, } \\
\text { suspended } \\
\text { in water } \\
\text { (mg/L) }\end{array}$ & $\begin{array}{c}\text { Total nitrogen } \\
\text { (nitrate + nitrite } \\
+ \text { ammonia + } \\
\text { organic-N), } \\
\text { unfiltered } \\
\text { (mg/L) }\end{array}$ & $\begin{array}{c}\text { Ortho- } \\
\text { phosphate, } \\
\text { filtered } \\
\text { (my/L as } \\
\text { phosphorus) }\end{array}$ & $\begin{array}{c}\text { Phosphorus. } \\
\text { unfiltered } \\
\text { (mg/L as } \\
\text { phosphorus) }\end{array}$ \\
\hline 1/9/2006 & - & 34.7 & $<0.04$ & $<0.06$ & $<0.008$ & - & 0.81 & $<0.006$ & 0.086 \\
\hline $3 / 6 / 2006$ & - & 10.9 & E0.03 & 0.23 & E0.006 & - & 1.31 & 0.027 & 0.26 \\
\hline $4 / 3 / 2006$ & - & 15.4 & E0.03 & 0.53 & 0.009 & - & 1.38 & 0.014 & 0.22 \\
\hline $5 / 2 / 2006$ & - & 7.47 & 0.08 & 1.05 & 0.025 & - & 1.99 & 0.051 & 0.40 \\
\hline $7 / 12 / 2006$ & - & 66.9 & 0.084 & 0.72 & 0.065 & - & 1.53 & 0.030 & 0.124 \\
\hline 7/18/2006 & - & - & - & - & - & - & - & - & - \\
\hline $8 / 1 / 2006$ & - & - & - & - & - & - & - & - & - \\
\hline $8 / 1 / 2006$ & - & - & - & - & - & - & - & - & - \\
\hline 9/12/2006 & - & 77.6 & 0.010 & $<0.06$ & $<0.002$ & - & 0.61 & 0.043 & 0.147 \\
\hline $10 / 24 / 2006$ & 11.5 & 23.9 & 0.099 & 0.07 & 0.010 & 0.09 & 1.26 & 0.128 & 0.32 \\
\hline $11 / 15 / 2006$ & 9.98 & 15.4 & 0.089 & 0.33 & 0.021 & 0.21 & 1.15 & 0.038 & 0.20 \\
\hline $12 / 4 / 2006$ & 10.9 & 33.5 & $<0.020$ & $<0.06$ & $<0.002$ & 0.35 & 0.68 & 0.033 & 0.09 \\
\hline 1/10/2007 & 6.34 & 5.83 & 0.057 & 0.32 & 0.010 & 0.91 & 1.24 & 0.096 & 0.32 \\
\hline 2/13/2007 & 7.39 & 10.9 & 0.062 & 0.35 & 0.005 & 0.53 & 1.27 & 0.058 & 0.30 \\
\hline 3/7/2007 & 7.96 & 18.2 & 0.164 & 1.14 & 0.042 & 0.51 & 2.54 & 0.012 & 0.22 \\
\hline $4 / 3 / 2007$ & 9.70 & 53.5 & E0.019 & 1.31 & 0.136 & 0.52 & 3.17 & E0.004 & 0.10 \\
\hline 4/14/2007 & 7.53 & 27.3 & 0.268 & 1.41 & 0.053 & 0.48 & 2.69 & 0.019 & 0.23 \\
\hline 4/16/2007 & 4.46 & 19.5 & 0.840 & 3.00 & 0.095 & 0.34 & 6.35 & 0.076 & 0.37 \\
\hline 4/30/2007 & 3.28 & 17.3 & Е0.013 & 1.08 & 0.043 & 0.36 & 1.99 & 0.013 & 0.15 \\
\hline $5 / 4 / 2007$ & 4.90 & 26.2 & 0.233 & 0.82 & 0.036 & 0.56 & 1.92 & 0.023 & 0.20 \\
\hline $5 / 8 / 2007$ & 6.11 & 19.4 & 0.201 & 1.29 & 0.125 & 0.57 & 2.41 & 0.056 & 0.24 \\
\hline $5 / 14 / 2007$ & 6.15 & 20.6 & 0.184 & 1.11 & 0.111 & 0.65 & 2.29 & 0.064 & 0.27 \\
\hline $5 / 17 / 2007$ & - & - & - & - & - & - & - & - & - \\
\hline $5 / 21 / 2007$ & 8.66 & 37.9 & E0.012 & 0.11 & 0.023 & 0.50 & 1.14 & E0.005 & 0.12 \\
\hline $5 / 30 / 2007$ & 6.26 & 75.0 & 0.087 & 0.34 & 0.032 & 0.17 & 1.21 & 0.018 & 0.09 \\
\hline 6/4/2007 & 9.92 & 78.8 & $<0.020$ & 0.43 & 0.054 & 0.17 & 1.34 & 0.013 & 0.10 \\
\hline 6/11/2007 & 13.3 & 93.5 & 0.051 & 0.89 & 0.098 & 0.32 & 1.79 & 0.027 & 0.11 \\
\hline 6/18/2007 & 14.9 & 90.3 & 0.071 & 0.55 & 0.083 & 0.15 & 1.50 & 0.059 & 0.12 \\
\hline 6/19/2007 & 7.36 & 26.9 & 0.309 & 2.72 & 0.077 & 1.36 & 5.42 & 0.070 & 0.51 \\
\hline 6/20/2007 & 7.49 & 28.3 & 0.546 & 1.11 & 0.112 & 0.66 & 4.00 & 0.084 & 0.38 \\
\hline $6 / 21 / 2007$ & 9.98 & 33.9 & 0.752 & 1.45 & 0.162 & 0.25 & 3.67 & 0.099 & 0.20 \\
\hline 6/25/2007 & 12.6 & 33.4 & 0.400 & 1.84 & 0.548 & 0.15 & 3.30 & 0.073 & 0.15 \\
\hline $7 / 2 / 2007$ & 7.30 & 19.8 & 0.088 & 1.00 & 0.093 & 0.28 & 1.92 & 0.103 & 0.29 \\
\hline $7 / 16 / 2007$ & 12.3 & 8.15 & 0.074 & 0.38 & 0.024 & 0.17 & 1.10 & 0.214 & 0.33 \\
\hline 7/30/2007 & 12.2 & 51.2 & 0.030 & 0.10 & 0.013 & 0.21 & 0.64 & 0.084 & 0.12 \\
\hline $8 / 6 / 2007$ & 14.0 & 60.0 & Е0.018 & E0.05 & 0.004 & 0.21 & 0.48 & 0.094 & 0.14 \\
\hline 8/20/2007 & 15.9 & 78.8 & E0.014 & $<0.06$ & 0.004 & 0.20 & 0.44 & 0.129 & 0.15 \\
\hline 8/28/2007 & 18.1 & 83.0 & $<0.020$ & $<0.06$ & E0.001 & 0.29 & 0.55 & 0.095 & 0.18 \\
\hline 9/4/2007 & 15.7 & 56.7 & 0.165 & E0.03 & 0.007 & 0.13 & 0.90 & 0.164 & 0.21 \\
\hline 9/11/2007 & 11.7 & 39.3 & 0.090 & 0.19 & 0.018 & 0.18 & 0.81 & 0.111 & 0.17 \\
\hline
\end{tabular}

Table 2. Surface-water-quality data for samples collected at station 07288650, Bogue Phalia near Leland, MS, 2006-2008. - Continued

[NGVD 29, National Geodetic Vertical Datum of 1929; mg/L, milligram per liter, $\mu \mathrm{g} / \mathrm{L}$, microgram per liter; -, no data; E, estimated; see fig. 1 for location]

\begin{tabular}{|c|c|c|c|c|c|c|c|c|c|}
\hline $\begin{array}{c}\text { Sample } \\
\text { date }\end{array}$ & $\begin{array}{c}\text { Carbon } \\
\text { (inorganic } \\
\text { plus organic), } \\
\text { suspended } \\
\text { sediment, } \\
\text { total } \\
\text { (mg/L) }\end{array}$ & $\begin{array}{c}\text { Inorganic } \\
\text { carbono, } \\
\text { suspended } \\
\text { sediment, } \\
\text { total } \\
\text { (mgl/L) }\end{array}$ & $\begin{array}{l}\text { Organic } \\
\text { carbon, } \\
\text { filtered } \\
\text { (mg/L) }\end{array}$ & $\begin{array}{l}\text { Iron, } \\
\text { filtered } \\
\text { (ng/L) }\end{array}$ & $\begin{array}{c}\text { Manganese, } \\
\text { filteredd } \\
(\mu / \mathrm{g} / \mathrm{L})\end{array}$ & $\begin{array}{c}\text { Aminomethyl- } \\
\text { phosphonoic } \\
\text { acidid } \\
\text { receverable } \\
\text { (yg/L) }\end{array}$ & $\begin{array}{c}\text { Glutosinate } \\
\text { (pg/L/L) }\end{array}$ & 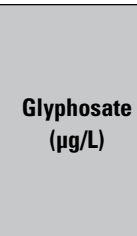 & $\begin{array}{c}\text { Suspended } \\
\text { sediment } \\
\text { concentration } \\
\text { (mg/L) }\end{array}$ \\
\hline 1/9/2006 & - & - & - & - & - & - & - & - & 70 \\
\hline $3 / 6 / 2006$ & - & - & - & - & - & 0.750 & $<0.140$ & 0.440 & 225 \\
\hline $4 / 3 / 2006$ & - & - & - & - & - & 1.00 & $<0.140$ & 0.390 & 140 \\
\hline $5 / 2 / 2006$ & - & - & - & - & - & 0.850 & $<0.140$ & 1.01 & 374 \\
\hline 7/12/2006 & - & - & - & - & - & 4.24 & $<0.140$ & 1.32 & 99 \\
\hline $7 / 18 / 2006$ & - & - & - & - & - & - & - & - & - \\
\hline $8 / 1 / 2006$ & - & - & - & - & - & - & - & - & - \\
\hline $8 / 1 / 2006$ & - & - & - & - & - & - & - & - & - \\
\hline 9/12/2006 & - & - & - & - & - & 1.32 & $<0.140$ & 0.450 & 107 \\
\hline $10 / 24 / 2006$ & 0.47 & $<0.12$ & 11.2 & 36 & 136 & 1.9 & $<0.10$ & 0.50 & 60 \\
\hline $11 / 15 / 2006$ & 1.11 & $<0.12$ & 7.3 & 12 & 56.0 & 1.3 & $<0.02$ & 0.21 & 129 \\
\hline $12 / 4 / 2006$ & 1.63 & $<0.12$ & 6.8 & 7 & 247 & 0.82 & $<0.02$ & 0.11 & 54 \\
\hline $1 / 10 / 2007$ & 4.63 & $<0.12$ & 6.6 & 22 & 5.5 & 0.80 & $<0.02$ & 0.25 & 214 \\
\hline 2/13/2007 & 2.69 & $<0.12$ & 5.5 & 11 & 56.3 & 0.68 & $<0.02$ & 0.26 & 176 \\
\hline 3/7/2007 & 2.21 & $<0.12$ & 6.2 & E6 & 112 & 0.88 & $<0.02$ & 0.34 & 177 \\
\hline $4 / 3 / 2007$ & 2.44 & $<0.12$ & 7.1 & 9 & 45.0 & 1.2 & $<0.02$ & 0.20 & 76 \\
\hline 4/14/2007 & 3.03 & $<0.12$ & 5.5 & E4 & 162 & 2.8 & $<0.02$ & 2.2 & 260 \\
\hline 4/16/2007 & 2.27 & 0.20 & 7.6 & 6 & 4.9 & 3.3 & 0.11 & 2.1 & 277 \\
\hline 4/30/2007 & 2.04 & $<0.12$ & 7.0 & E3 & 20.0 & 1.7 & $<0.02$ & 0.28 & 104 \\
\hline $5 / 4 / 2007$ & 3.38 & $<0.12$ & 6.8 & 7 & 47.6 & 2.0 & $<0.02$ & 0.84 & 190 \\
\hline $5 / 8 / 2007$ & 2.35 & $<0.12$ & 6.8 & 8 & 61.8 & 2.6 & 0.12 & 0.56 & 181 \\
\hline $5 / 14 / 2007$ & 2.75 & $<0.12$ & 7.2 & E3 & 85.6 & 4.3 & 3.2 & 2.1 & 154 \\
\hline $5 / 17 / 2007$ & - & - & 7.0 & - & - & - & - & - & - \\
\hline $5 / 21 / 2007$ & 2.19 & $<0.12$ & 7.1 & E5 & 133 & 2.9 & $<0.02$ & 0.66 & 63 \\
\hline $5 / 30 / 2007$ & 0.81 & $<0.12$ & 5.5 & 19 & 183 & 3.5 & $<0.02$ & 1.1 & 86 \\
\hline 6/4/2007 & 1.01 & $<0.12$ & 6.3 & 8 & 19.5 & 6.7 & $<0.02$ & 1.9 & 81 \\
\hline 6/11/2007 & 1.84 & $<0.12$ & 7.0 & E4 & 19.2 & 6.8 & $<0.02$ & 1.5 & 89 \\
\hline 6/18/2007 & 1.11 & $<0.12$ & 6.8 & E4 & 26.8 & 28 & $<0.02$ & 15 & 76 \\
\hline 6/19/2007 & 8.53 & $<0.12$ & 5.9 & 6 & 0.9 & 4.8 & $<0.02$ & 4.2 & 662 \\
\hline 6/20/2007 & 4.21 & $<0.12$ & 6.7 & 10 & 8.2 & 3.5 & $<0.02$ & 2.1 & 322 \\
\hline 6/21/2007 & 1.90 & $<0.12$ & 9.8 & 10 & 12.4 & 3.5 & $<0.02$ & 2.0 & 113 \\
\hline 6/25/2007 & 0.88 & $<0.12$ & 5.7 & 29 & 20.0 & 2.9 & $<0.02$ & 0.97 & 83 \\
\hline $7 / 2 / 2007$ & 1.31 & $<0.12$ & 5.6 & 13 & 20.6 & 4.0 & $<0.02$ & 2.9 & 203 \\
\hline $7 / 16 / 2007$ & 1.26 & $<0.12$ & 5.5 & 25 & 27.1 & 2.7 & $<0.02$ & 1.3 & 64 \\
\hline $7 / 30 / 2007$ & 1.01 & $<0.12$ & 5.1 & $<6$ & 23.2 & 3.3 & $<0.02$ & 1.0 & 75 \\
\hline $8 / 6 / 2007$ & 1.42 & $<0.12$ & 5.5 & E5 & 25.2 & 4.2 & $<0.02$ & 1.0 & 86 \\
\hline 8/20/2007 & 0.94 & $<0.12$ & 5.7 & 20 & 20.2 & 4.1 & $<0.02$ & 0.92 & 117 \\
\hline $8 / 28 / 2007$ & 1.74 & $<0.12$ & 6.2 & E5 & 44.6 & 3.7 & $<0.02$ & 1.4 & 167 \\
\hline 9/4/2007 & 1.10 & $<0.12$ & 7.8 & 9 & 90.9 & 4.0 & $<0.02$ & 1.1 & 117 \\
\hline 9/11/2007 & 1.29 & $<0.12$ & 6.5 & 8 & 9.7 & 3.6 & $<0.02$ & 0.38 & 95 \\
\hline
\end{tabular}


Table 2. Surface-water-quality data for samples collected at station 07288650, Bogue Phalia near Leland, MS, 2006-2008. -Continued

[NGVD 29, National Geodetic Vertical Datum of 1929; mg/L, milligram per liter; $\mu \mathrm{g} / \mathrm{L}$, microgram per liter, -, no data; E, estimated; see fig. 1 for location]

\begin{tabular}{|c|c|c|c|c|c|c|c|c|}
\hline $\begin{array}{c}\text { Sample } \\
\text { date }\end{array}$ & $\begin{array}{l}\text { Water-surface } \\
\text { elevation } \\
\text { (feet above } \\
\text { NGVD 29) }\end{array}$ & $\begin{array}{c}\text { Gage } \\
\text { height } \\
\text { (feet) }\end{array}$ & $\begin{array}{l}\text { Discharge } \\
\text { (cubici feet } \\
\text { per second) }\end{array}$ & $\begin{array}{l}\text { Turbiditity in } \\
\text { Nephelometric } \\
\text { Turbidity Units }\end{array}$ & $\begin{array}{c}\text { Dissolved } \\
\text { oxygen } \\
\text { (mg/L) }\end{array}$ & pH & $\begin{array}{c}\text { Specific } \\
\text { conductance, } \\
\text { (microsiemens } \\
\text { per centimeter) }\end{array}$ & $\begin{array}{c}\text { Water } \\
\text { temperature } \\
\text { (degress } \\
\text { Celsius) }\end{array}$ \\
\hline 10/19/2007 & 94.86 & 8.65 & 192 & 23 & 6.1 & 7.7 & 413 & 24.2 \\
\hline $11 / 26 / 2007$ & 94.31 & 8.10 & 135 & 58 & 9.2 & 7.5 & 367 & 10.8 \\
\hline $11 / 27 / 2007$ & 95.80 & 9.59 & 322 & 180 & 8.5 & 7.2 & 236 & 10.7 \\
\hline $12 / 3 / 2007$ & 92.97 & 6.76 & 43 & - & 9.1 & 7.8 & 312 & 12.6 \\
\hline $12 / 5 / 2007$ & 92.75 & 6.54 & 17 & - & 8.9 & 7.5 & 317 & 11.3 \\
\hline 2/1/2008 & 103.91 & 17.70 & 2,770 & $>1,000$ & 11.8 & 6.4 & 112 & 4.5 \\
\hline 2/5/2008 & 102.80 & 16.59 & 2,260 & 620 & 9.1 & 7.0 & 111 & 12.7 \\
\hline $2 / 27 / 2008$ & 96.71 & 10.50 & 482 & $>1,000$ & 9.9 & 7.3 & 142 & 9.0 \\
\hline 3/4/2008 & 106.85 & 20.64 & 4,160 & $>1,000$ & 9.0 & 7.7 & 63 & 12.0 \\
\hline $3 / 5 / 2008$ & 108.64 & 22.43 & 5,220 & $>1,000$ & 8.8 & 6.9 & 58 & 10.3 \\
\hline 3/6/2008 & 106.06 & 19.85 & 3,600 & $>1,000$ & 9.0 & 7.0 & 69 & 10.5 \\
\hline 3/11/2008 & 95.56 & 9.35 & 284 & 400 & 9.6 & 7.1 & 95 & 12.0 \\
\hline 4/2/2008 & 106.91 & 20.70 & 4,520 & - & 6.9 & 6.8 & 92 & 16.8 \\
\hline 4/7/2008 & 108.20 & 21.99 & 5,360 & 350 & 6.5 & 6.8 & 74 & 16.0 \\
\hline 4/22/2008 & 92.90 & 6.69 & 25 & - & 7.4 & 7.7 & 231 & 22.3 \\
\hline $4 / 30 / 2008$ & 100.54 & 14.33 & 1,380 & 540 & 7.0 & 6.7 & 106 & 14.3 \\
\hline $5 / 6 / 2008$ & 107.98 & 21.77 & 5,230 & 520 & - & - & - & - \\
\hline $5 / 13 / 2008$ & 86.21 & - & - & - & - & 7.2 & - & - \\
\hline $5 / 16 / 2008$ & 106.54 & 20.33 & 4,270 & 710 & 7.7 & 6.9 & 82 & 22.0 \\
\hline $5 / 20 / 2008$ & 95.90 & 9.69 & 330 & 150 & 6.4 & 7.1 & 140 & 27.2 \\
\hline $5 / 27 / 2008$ & 96.27 & 10.06 & 399 & 300 & 5.0 & 6.8 & 113 & 34.0 \\
\hline 6/3/2008 & 92.99 & 6.78 & 30 & - & 6.6 & 7.3 & 222 & 31.4 \\
\hline 6/10/2008 & 93.56 & 7.35 & 68 & 39 & 7.4 & 7.7 & 536 & 30.7 \\
\hline 6/17/2008 & 94.22 & 8.01 & 124 & 40 & 9.1 & 8.1 & 419 & 31.2 \\
\hline 6/24/2008 & 93.70 & 7.49 & 84 & - & 7.7 & 7.7 & 577 & 28.9 \\
\hline 6/26/2008 & 93.91 & 7.70 & 103 & - & 6.0 & 7.9 & 566 & 28.6 \\
\hline $7 / 1 / 2008$ & 94.11 & 7.90 & 116 & 53 & 13.7 & 6.4 & 481 & 27.5 \\
\hline $7 / 8 / 2008$ & 95.58 & 9.37 & 290 & 25 & 5.9 & 6.8 & 479 & 30.2 \\
\hline 7/15/2008 & 93.91 & 7.70 & 95 & 42 & 6.6 & 7.7 & 530 & 30.0 \\
\hline $7 / 23 / 2008$ & 94.59 & 8.38 & 160 & 31 & 6.5 & 7.5 & 636 & 31.4 \\
\hline $8 / 14 / 2008$ & 101.36 & 15.15 & 1,690 & 30 & 5.3 & 6.4 & 361 & 25.6 \\
\hline $8 / 18 / 2008$ & 97.34 & 11.13 & 615 & - & 7.0 & 7.4 & 363 & 26.7 \\
\hline $9 / 4 / 2008$ & 111.86 & 25.65 & 6,290 & 54 & 5.0 & 6.7 & 119 & 24.3 \\
\hline 9/30/2008 & 92.93 & 6.45 & 27 & 16 & 5.8 & 6.6 & 280 & 24.6 \\
\hline $10 / 14 / 2008$ & 92.60 & 6.39 & 15 & 9 & 6.4 & 6.9 & 384 & 23.7 \\
\hline $11 / 3 / 2008$ & 92.61 & 6.36 & 8.5 & 10 & 8.7 & 8.3 & 506 & 16.2 \\
\hline $11 / 6 / 2008$ & 92.61 & 6.37 & 8.3 & 12 & 7.6 & 7.1 & 519 & 17.2 \\
\hline
\end{tabular}

Table 2. Surface-water-quality data for samples collected at station 07288650, Bogue Phalia near Leland, MS, 2006-2008. -Continued

[NGVD 29, National Geodetic Vertical Datum of 1929; mg/L, milligram per liter; $\mu \mathrm{g} / \mathrm{L}$, microgram per liter; -, no data; E, estimated; see fig. 1 for location]

\begin{tabular}{|c|c|c|c|c|c|c|c|c|}
\hline $\begin{array}{c}\text { Sample } \\
\text { date }\end{array}$ & 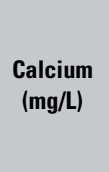 & $\begin{array}{c}\text { Magnesium } \\
(\mathrm{m} y / /)\end{array}$ & $\begin{array}{c}\text { Potasssium } \\
\text { (my/L) }\end{array}$ & $\begin{array}{c}\text { Sodium } \\
\text { (mg/L) }\end{array}$ & $\begin{array}{l}\text { Alkalinity, } \\
\text { field, } \\
\text { (myll/ as } \\
\text { calcium } \\
\text { carbonate) }\end{array}$ & $\begin{array}{c}\text { Bicarbonate, } \\
\text { field } \\
\text { (mg/L) }\end{array}$ & $\begin{array}{c}\text { Chloride } \\
\text { (mgl/L) }\end{array}$ & $\begin{array}{l}\text { Fluoride } \\
(\mathrm{mg} / \mathrm{L})\end{array}$ \\
\hline 10/19/2007 & 39.9 & 17.2 & 5.83 & 15.4 & - & - & 9.42 & 0.21 \\
\hline $11 / 26 / 2007$ & 42.9 & 13.4 & 5.55 & 13.1 & 145 & 175 & 7.92 & 0.15 \\
\hline $11 / 27 / 2007$ & 21.6 & 7.14 & 7.77 & 8.80 & 72 & 87 & 9.56 & 0.14 \\
\hline $12 / 3 / 2007$ & 30.6 & 10.2 & 7.13 & 11.5 & 109 & 132 & 12.7 & 0.19 \\
\hline $12 / 5 / 2007$ & - & - & - & - & - & - & - & - \\
\hline 2/1/2008 & 10.6 & 3.29 & 3.05 & 5.05 & 33 & 40 & 4.01 & 0.14 \\
\hline 2/5/2008 & 11.5 & 3.56 & 3.36 & 4.39 & 43 & 52 & 2.93 & E0.10 \\
\hline 2/27/2008 & 14.2 & 3.98 & 3.29 & 6.91 & 54 & 65 & 3.60 & 0.13 \\
\hline 3/4/2008 & 6.01 & 1.77 & 2.07 & 2.46 & 26 & 31 & 1.50 & Е0.11 \\
\hline 3/5/2008 & 5.27 & 1.55 & 2.02 & 2.19 & 19 & 23 & 1.26 & Е0.09 \\
\hline 3/6/2008 & - & - & - & - & - & - & - & - \\
\hline $3 / 11 / 2008$ & 9.60 & 2.89 & 3.32 & 3.04 & 35 & 42 & 1.89 & E0.10 \\
\hline 4/2/2008 & 9.09 & 2.69 & 2.91 & 3.60 & 27 & 33 & 2.37 & Е0.12 \\
\hline 4/7/2008 & 7.62 & 2.22 & 2.64 & 2.74 & 28 & 34 & 1.40 & Е0.11 \\
\hline 4/22/2008 & 25.3 & 7.85 & 3.53 & 6.50 & 97 & 117 & 3.89 & 0.16 \\
\hline 4/30/2008 & 10.5 & 3.09 & 3.08 & 3.36 & 26 & 32 & 2.17 & Е0.12 \\
\hline $5 / 6 / 2008$ & - & - & - & - & 43 & 53 & - & - \\
\hline $5 / 13 / 2008$ & 11.6 & 3.35 & 3.67 & 2.34 & 43 & 53 & 1.54 & Е0.09 \\
\hline $5 / 16 / 2008$ & 6.80 & 2.05 & 2.53 & 2.33 & 19 & 23 & 1.09 & 0.10 \\
\hline $5 / 20 / 2008$ & 13.7 & 3.94 & 3.39 & 3.82 & 35 & 42 & 2.11 & 0.14 \\
\hline $5 / 27 / 2008$ & 10.7 & 3.12 & 2.92 & 3.47 & 33 & 40 & 1.76 & 0.15 \\
\hline 6/3/2008 & 22.7 & 6.78 & 3.54 & 5.11 & 76 & 93 & 2.44 & 0.18 \\
\hline 6/10/2008 & 57.1 & 18.2 & 4.34 & 19.1 & 164 & 198 & 8.53 & 0.21 \\
\hline 6/17/2008 & 44.5 & 14.3 & 4.10 & 14.0 & 145 & 173 & 7.76 & 0.21 \\
\hline 6/24/2008 & 58.9 & 23.7 & 4.16 & 25.0 & 197 & 237 & 11.1 & 0.20 \\
\hline 6/26/2008 & - & - & - & - & - & - & - & - \\
\hline 7/1/2008 & 48.2 & 19.3 & 4.03 & 21.6 & 156 & 185 & 9.95 & 0.23 \\
\hline 7/8/2008 & 49.3 & 18.5 & 4.86 & 18.8 & 167 & 200 & 8.48 & 0.26 \\
\hline 7/15/2008 & 54.3 & 22.0 & 3.81 & 20.5 & 201 & 242 & 7.47 & 0.27 \\
\hline 7/23/2008 & 71.7 & 28.0 & 4.04 & 28.5 & 232 & 277 & 11.8 & 0.25 \\
\hline 8/14/2008 & 38.6 & 13.6 & 5.01 & 12.3 & 145 & 175 & 6.13 & 0.18 \\
\hline 8/18/2008 & 38.3 & 13.2 & 5.32 & 12.4 & 150 & 181 & 6.92 & 0.19 \\
\hline 9/4/2008 & 11.8 & 3.48 & 5.17 & 2.76 & 45 & 55 & 2.36 & E0.09 \\
\hline 9/30/2008 & - & - & - & - & - & - & - & - \\
\hline $10 / 14 / 2008$ & 50.6 & 14.8 & 4.46 & 7.70 & 174 & E212 & 4.12 & 0.16 \\
\hline $11 / 3 / 2008$ & 68.5 & 19.9 & 3.49 & 13.8 & 215 & 260 & 7.32 & 0.20 \\
\hline $11 / 6 / 2008$ & - & - & - & - & - & - & - & - \\
\hline
\end{tabular}


Table 2. Surface-water-quality data for samples collected at station 07288650, Bogue Phalia near Leland, MS, 2006-2008. - Continued

[NGVD 29, National Geodetic Vertical Datum of 1929; mg/L, milligram per liter, $\mu \mathrm{g} / \mathrm{L}$, microgram per liter, -, no data; E, estimated; see fig. 1 for location]

\begin{tabular}{|c|c|c|c|c|c|c|c|c|c|}
\hline $\begin{array}{c}\text { Sample } \\
\text { date }\end{array}$ & $\begin{array}{c}\text { Silica } \\
\text { (imgl as } \\
\text { silica } \\
\text { dioxide) }\end{array}$ & $\begin{array}{l}\text { Sulfate } \\
\text { (mg/L) }\end{array}$ & $\begin{array}{c}\text { Ammonia, } \\
\text { filtered } \\
\text { (mg// as } \\
\text { nitrogen) }\end{array}$ & $\begin{array}{l}\text { Nitrate } \\
\text { plus intrite, } \\
\text { filtered, } \\
\text { (mygl/ as } \\
\text { nitrogen) }\end{array}$ & $\begin{array}{l}\text { Nitrite, } \\
\text { filtered } \\
\text { (my/l/ as } \\
\text { nitrogen) }\end{array}$ & $\begin{array}{c}\text { Particulate } \\
\text { nitrogen, } \\
\text { suspended } \\
\text { in water } \\
\text { (mg/L) }\end{array}$ & $\begin{array}{c}\text { Total nitrogen } \\
\text { (nitrate + +nitrite } \\
+ \text { ammonia }+ \\
\text { organic-N). } \\
\text { unfiltered } \\
\text { (mg/L) }\end{array}$ & $\begin{array}{c}\text { Ortho- } \\
\text { phosphate, } \\
\text { fitered } \\
\text { (my/L as } \\
\text { phosphorus) }\end{array}$ & $\begin{array}{l}\text { Phosphorus } \\
\text { unfiltered } \\
\text { (mg/las } \\
\text { phosphorus }\end{array}$ \\
\hline $10 / 19 / 2007$ & 9.68 & 38.9 & 0.082 & 0.05 & 0.008 & 0.41 & 0.67 & 0.081 & 0.15 \\
\hline $11 / 26 / 2007$ & 8.41 & 29.1 & 0.038 & 0.51 & 0.005 & 0.28 & 1.07 & 0.041 & 0.14 \\
\hline $11 / 27 / 2007$ & 8.22 & 22.4 & 0.027 & 1.28 & 0.014 & 0.49 & 2.25 & 0.128 & 0.30 \\
\hline $12 / 3 / 2007$ & 7.53 & 21.7 & 0.057 & 0.38 & 0.009 & 0.14 & 1.10 & 0.068 & 0.16 \\
\hline $12 / 5 / 2007$ & - & - & - & - & - & - & - & - & - \\
\hline $2 / 1 / 2008$ & 4.90 & 10.4 & 0.063 & 0.82 & 0.006 & 1.39 & 2.40 & 0.055 & 0.50 \\
\hline $2 / 5 / 2008$ & 8.43 & 7.75 & Е0.082 & 0.57 & 0.017 & 1.01 & 1.78 & 0.156 & 0.49 \\
\hline 2/27/2008 & 6.07 & 8.28 & 0.158 & 0.53 & 0.014 & 1.65 & 2.29 & 0.124 & 0.59 \\
\hline $3 / 4 / 2008$ & 4.45 & 4.55 & 0.088 & 0.38 & 0.008 & 1.48 & 2.67 & 0.046 & 0.65 \\
\hline $3 / 5 / 2008$ & 4.03 & 3.76 & 0.104 & 0.42 & 0.009 & 1.33 & 2.22 & 0.070 & 0.53 \\
\hline $3 / 6 / 2008$ & - & - & - & - & - & - & - & - & - \\
\hline 3/11/2008 & 5.38 & 4.31 & 0.172 & 0.63 & 0.016 & 0.74 & 2.33 & 0.105 & 0.46 \\
\hline 4/2/2008 & 5.49 & 7.76 & 0.137 & 1.19 & 0.032 & 1.31 & 2.97 & 0.080 & 0.52 \\
\hline 4/7/2008 & 5.61 & 5.11 & 0.040 & 0.43 & 0.014 & 0.79 & 1.35 & 0.095 & 0.33 \\
\hline $4 / 22 / 2008$ & 8.61 & 16.8 & 0.043 & 0.06 & 0.004 & 0.31 & 0.79 & 0.048 & 0.17 \\
\hline $4 / 30 / 2008$ & 6.90 & 8.64 & 0.142 & 1.43 & 0.042 & 0.84 & 2.76 & 0.103 & 0.40 \\
\hline $5 / 6 / 2008$ & - & - & - & - & - & - & - & - & - \\
\hline $5 / 13 / 2008$ & 7.50 & 4.40 & 0.096 & 0.57 & 0.035 & 0.30 & 1.29 & 0.167 & 0.35 \\
\hline $5 / 16 / 2008$ & 5.73 & 6.95 & 0.357 & 1.85 & 0.057 & 0.97 & 3.36 & 0.061 & 0.34 \\
\hline $5 / 20 / 2008$ & 7.64 & 13.8 & 0.350 & 2.29 & 0.262 & 0.32 & 4.04 & 0.081 & 0.26 \\
\hline $5 / 27 / 2008$ & 6.37 & 8.84 & 0.175 & 1.49 & 0.186 & 0.51 & 2.65 & 0.079 & 0.32 \\
\hline 6/3/2008 & 10.2 & 17.6 & 0.126 & 1.47 & 0.167 & 0.42 & 2.85 & 0.040 & 0.17 \\
\hline 6/10/2008 & 14.3 & 72.3 & 0.032 & 1.22 & 0.073 & 0.42 & 1.89 & 0.045 & 0.14 \\
\hline 6/17/2008 & 11.4 & 51.3 & $<0.020$ & 0.66 & 0.071 & 0.60 & 1.59 & 0.025 & 0.14 \\
\hline 6/24/2008 & 10.8 & 94.2 & 0.058 & 0.13 & 0.025 & 0.39 & 0.82 & 0.031 & 0.11 \\
\hline 6/26/2008 & - & - & - & - & - & - & - & - & - \\
\hline $7 / 1 / 2008$ & 5.16 & 71.2 & $<0.020$ & 0.75 & 0.198 & 0.76 & 1.89 & $<0.006$ & 0.11 \\
\hline $7 / 8 / 2008$ & 10.8 & 59.2 & 0.029 & 0.42 & 0.040 & 0.32 & 1.22 & 0.033 & 0.11 \\
\hline $7 / 15 / 2008$ & 12.7 & 66.8 & 0.086 & 0.33 & 0.039 & 0.35 & 1.23 & 0.047 & 0.13 \\
\hline $7 / 23 / 2008$ & 13.5 & 82.9 & 0.027 & E0.03 & 0.004 & 0.30 & 0.60 & 0.061 & 0.12 \\
\hline $8 / 14 / 2008$ & 14.9 & 29.1 & Е0.013 & 0.44 & 0.025 & 0.21 & 1.16 & 0.114 & 0.19 \\
\hline $8 / 18 / 2008$ & 16.1 & 24.3 & 0.021 & 0.28 & 0.022 & 0.18 & 0.91 & 0.123 & 0.18 \\
\hline 9/4/2008 & 8.59 & 7.38 & Е0.016 & 0.23 & 0.012 & 0.21 & 0.97 & 0.160 & 0.27 \\
\hline 9/30/2008 & - & - & - & - & - & - & - & - & - \\
\hline $10 / 14 / 2008$ & 15.7 & 19.7 & $<0.020$ & $<0.04$ & E0.001 & 0.09 & 0.28 & 0.038 & 0.07 \\
\hline $11 / 3 / 2008$ & 14.0 & 47.7 & $<0.020$ & $<0.04$ & $<0.002$ & 0.10 & 0.35 & 0.015 & 0.05 \\
\hline 11/6/2008 & - & - & & & & 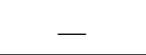 & 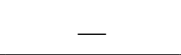 & 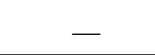 & - \\
\hline
\end{tabular}

Table 2. Surface-water-quality data for samples collected at station 07288650, Bogue Phalia near Leland, MS, 2006-2008. - Continued

[NGVD 29, National Geodetic Vertical Datum of 1929; mg/L, milligram per liter; $\mu \mathrm{g} / \mathrm{L}$, microgram per liter; -, no data; E, estimated; see fig. 1 for location]

\begin{tabular}{|c|c|c|c|c|c|c|c|c|c|}
\hline $\begin{array}{c}\text { Sample } \\
\text { date }\end{array}$ & $\begin{array}{c}\text { Carbon } \\
\text { (inorganic } \\
\text { plus organic), } \\
\text { suspended, } \\
\text { sediment, } \\
\text { total } \\
\text { (mg/L) }\end{array}$ & $\begin{array}{c}\text { Inorganic } \\
\text { carbon, } \\
\text { suspended } \\
\text { sediment, } \\
\text { total } \\
\text { (mg/L) }\end{array}$ & $\begin{array}{l}\text { Organic } \\
\text { carbon, } \\
\text { fittered } \\
\text { (mg/L) }\end{array}$ & $\begin{array}{l}\text { Iron, } \\
\text { fitered } \\
\text { (pg/L/) }\end{array}$ & $\begin{array}{l}\text { Manganese, } \\
\text { fitered } \\
\text { (pg/L) }\end{array}$ & $\begin{array}{c}\text { Aminomethyl- } \\
\text { phosphonoic } \\
\text { acidi } \\
\text { receverable } \\
\text { (ygl/L) }\end{array}$ & $\begin{array}{c}\text { Glufosinate } \\
(\mathrm{pg} / \mathrm{L} / \mathrm{c}\end{array}$ & $\begin{array}{c}\text { Glyphosate } \\
(\mathrm{H} / / / \mathrm{c})\end{array}$ & $\begin{array}{c}\text { Suspended } \\
\text { sediment } \\
\text { concentration } \\
\text { (mg/L) }\end{array}$ \\
\hline $10 / 19 / 2007$ & 3.80 & $<0.04$ & 6.5 & $<8$ & 1.4 & 12 & $<0.02$ & 73 & 81 \\
\hline $11 / 26 / 2007$ & 2.30 & E0.03 & 6.0 & 12 & 56.5 & 2.6 & $<0.02$ & 1.0 & 84 \\
\hline $11 / 27 / 2007$ & 2.67 & E0.04 & 8.5 & 22 & 2.7 & 2.4 & $<0.02$ & 1.2 & 172 \\
\hline $12 / 3 / 2007$ & 0.93 & E0.05 & 8.5 & 10 & 19.4 & 2.5 & $<0.02$ & 1.2 & 83 \\
\hline $12 / 5 / 2007$ & - & - & 7.9 & - & - & - & - & - & 81 \\
\hline 2/1/2008 & 9.69 & 0.30 & 5.8 & 11 & 10.4 & 0.97 & $<0.02$ & 0.58 & 781 \\
\hline 2/5/2008 & 5.53 & $<0.04$ & 7.8 & - & 15.6 & 0.75 & $<0.02$ & 0.24 & 419 \\
\hline 2/27/2008 & 9.31 & E0.08 & 6.4 & 18 & 1.2 & 1.4 & $<0.02$ & 1.4 & 650 \\
\hline 3/4/2008 & 8.88 & 0.20 & 5.0 & 49 & 9.4 & 0.79 & $<0.02$ & 1.2 & 1,010 \\
\hline $3 / 5 / 2008$ & 7.76 & 0.19 & 4.7 & 67 & 5.5 & 0.93 & $<0.02$ & 0.57 & 648 \\
\hline 3/6/2008 & - & - & 6.3 & - & - & - & - & - & - \\
\hline $3 / 11 / 2008$ & 13.2 & 0.15 & 7.4 & 23 & 6.8 & 1.0 & $<0.02$ & 0.49 & 309 \\
\hline 4/2/2008 & 8.68 & $<0.04$ & 8.2 & 41 & 4.2 & 1.4 & $<0.02$ & 1.1 & 588 \\
\hline 4/7/2008 & 5.43 & $<0.04$ & 8.1 & 42 & 3.0 & 1.1 & $<0.02$ & 1.0 & 249 \\
\hline 4/22/2008 & 1.58 & $<0.04$ & 7.0 & 14 & 85.1 & 1.2 & $<0.02$ & 0.40 & 67 \\
\hline $4 / 30 / 2008$ & 5.10 & E0.07 & 8.3 & 23 & 5.2 & 1.4 & 0.16 & 0.66 & 333 \\
\hline $5 / 6 / 2008$ & - & - & E40.7 & - & - & 1.1 & $<0.02$ & 0.91 & - \\
\hline $5 / 13 / 2008$ & 2.40 & E0.10 & 6.3 & 30 & 42.3 & 1.5 & $<0.02$ & 0.48 & 117 \\
\hline $5 / 16 / 2008$ & 6.21 & $<0.04$ & 5.5 & 30 & 2.7 & 1.6 & $<0.02$ & 1.0 & 418 \\
\hline $5 / 20 / 2008$ & 1.78 & $<0.04$ & 6.9 & 21 & 21.6 & 1.6 & $<0.02$ & 0.85 & 139 \\
\hline $5 / 27 / 2008$ & 2.83 & $<0.04$ & 5.4 & 17 & 8.4 & 1.3 & $<0.02$ & 0.50 & 260 \\
\hline 6/3/2008 & 2.25 & $<0.04$ & 5.8 & E6 & 117 & 1.6 & $<0.02$ & 0.46 & 98 \\
\hline 6/10/2008 & 2.42 & $<0.04$ & 5.1 & E5 & 94.1 & 2.9 & $<0.02$ & 1.1 & 105 \\
\hline 6/17/2008 & 3.41 & $<0.04$ & 6.2 & 9 & 25.1 & 3.0 & $<0.02$ & 0.95 & 102 \\
\hline 6/24/2008 & 2.03 & $<0.04$ & 5.1 & E6 & 35.6 & 3.6 & $<0.02$ & 0.84 & 112 \\
\hline 6/26/2008 & - & - & 5.4 & - & - & - & - & - & 133 \\
\hline $7 / 1 / 2008$ & 4.72 & 0.19 & 6.6 & E7 & 1.7 & 3.0 & $<0.02$ & 1.3 & 127 \\
\hline $7 / 8 / 2008$ & 2.17 & E0.04 & 6.8 & E5 & 7.4 & 2.3 & $<0.02$ & 0.65 & 86 \\
\hline 7/15/2008 & 2.30 & 0.13 & 5.9 & E5 & 14.2 & 4.4 & $<0.02$ & 0.92 & 96 \\
\hline $7 / 23 / 2008$ & 2.08 & E0.04 & 5.2 & E6 & 8.5 & 5.3 & $<0.02$ & 18 & 126 \\
\hline $8 / 14 / 2008$ & 1.97 & 0.26 & 8.5 & 25 & 4.4 & 0.48 & $<0.02$ & 0.08 & 88 \\
\hline $8 / 18 / 2008$ & 1.40 & $<0.04$ & 6.5 & 20 & 3.9 & 3.2 & $<0.02$ & 1.5 & 75 \\
\hline $9 / 4 / 2008$ & 1.70 & $<0.04$ & 7.5 & 77 & 10 & 2.2 & $<0.02$ & 1.2 & 164 \\
\hline 9/30/2008 & - & - & 5.9 & - & - & - & - & - & - \\
\hline $10 / 14 / 2008$ & 0.58 & $<0.04$ & 4.0 & 6 & 216 & 2.3 & $<0.02$ & 0.22 & 134 \\
\hline $11 / 3 / 2008$ & 0.62 & $<0.04$ & 4.0 & 14 & 176 & 4.7 & $<0.02$ & 0.19 & 76 \\
\hline $11 / 6 / 2008$ & - & - & 3.8 & - & - & - & - & - & - \\
\hline
\end{tabular}




\section{Data Associated with the Mississippi Embayment Agricultural Chemical Transport Study, 2006-2008}

Table 3. Groundwater-quality data for samples collected at the flowpath along the Bogue Phalia near Leland, MS, 2006-2008. [NGVD 29, National Geodetic Vertical Datum of 1929; mg/L, milligram per liter; $\mu$ g/L, microgram per liter; site type: HW—-hyporheic zone,

\begin{tabular}{|c|c|c|c|c|c|c|c|c|c|}
\hline $\begin{array}{l}\text { Station } \\
\text { iddentifier }\end{array}$ & $\begin{array}{c}\text { Station } \\
\text { name } \\
\text { (fig. and } \\
\text { table 1) }\end{array}$ & $\begin{array}{l}\text { Site } \\
\text { type }\end{array}$ & $\begin{array}{c}\begin{array}{c}\text { Sample } \\
\text { date }\end{array} \\
\text { a }\end{array}$ & $\begin{array}{l}\text { Water level } \\
\text { (fiet below } \\
\text { land surface) }\end{array}$ & $\begin{array}{l}\text { Water-surface } \\
\text { elevation } \\
\text { (feet above } \\
\text { GGVD 29) }\end{array}$ & $\begin{array}{c}\text { Turbidity, in } \\
\text { Nephelometric } \\
\text { Turbididy Units }\end{array}$ & $\begin{array}{c}\text { Dissolved } \\
\text { oxygen } \\
(\mathrm{mg} / L)\end{array}$ & $\mathrm{pH}$ & $\begin{array}{c}\text { Specific } \\
\text { conductance, } \\
\text { (microsiemens } \\
\text { per centimeter }\end{array}$ \\
\hline \multirow[t]{13}{*}{332440090502001} & RB & $\mathrm{HW}$ & $12 / 5 / 2006$ & - & - & 0.6 & 0.4 & 6.9 & 738 \\
\hline & & & $2 / 13 / 2007$ & - & - & 0.2 & - & 7.0 & 528 \\
\hline & & & $5 / 15 / 2007$ & - & - & 0.5 & 0.3 & 6.8 & 758 \\
\hline & & & 6/26/2007 & - & - & 0.2 & 0.3 & 6.8 & 809 \\
\hline & & & 7/18/2007 & - & - & 0.7 & 0.2 & 6.7 & 672 \\
\hline & & & 8/7/2007 & - & - & 0.8 & 0.2 & 6.8 & 579 \\
\hline & & & $12 / 3 / 2007$ & - & - & 0.3 & 0.5 & 6.8 & 549 \\
\hline & & & $12 / 4 / 2007$ & 3.53 & - & 20 & 0.4 & 6.4 & 764 \\
\hline & & & 2/27/2008 & - & - & 0.1 & 0.3 & 6.9 & 675 \\
\hline & & & 3/6/2008 & - & - & 0.3 & 0.5 & 6.9 & 754 \\
\hline & & & 4/10/2008 & - & - & 1.2 & 0.3 & 7.0 & 728 \\
\hline & & & 6/23/2008 & - & - & 0.2 & 0.3 & 6.8 & 538 \\
\hline & & & 6/23/2008 & 0.69 & - & - & 1.1 & 7.1 & 514 \\
\hline \multirow[t]{6}{*}{332440090502195} & $\mathrm{RC}$ & $\mathrm{HW}$ & 12/5/2006 & - & - & 1.5 & - & 6.9 & 690 \\
\hline & & & 2/13/2007 & - & - & 7.4 & - & 7.1 & 741 \\
\hline & & & 5/15/2007 & - & - & 4.0 & 0.6 & 6.8 & 777 \\
\hline & & & $12 / 5 / 2007$ & - & - & 11 & 0.2 & 6.7 & 737 \\
\hline & & & 6/25/2008 & - & - & 4.6 & 0.2 & 6.8 & 778 \\
\hline & & & $11 / 4 / 2008$ & - & - & 64 & 0.8 & 6.8 & 773 \\
\hline \multirow[t]{4}{*}{332440090502196} & C & $\mathrm{HW}$ & 8/8/2007 & - & - & 13 & 0.5 & 6.7 & 641 \\
\hline & & & $12 / 5 / 2007$ & - & - & 11 & 0.7 & 6.8 & 682 \\
\hline & & & 6/25/2008 & -2.56 & - & 1.4 & 0.8 & 6.8 & 458 \\
\hline & & & $11 / 4 / 2008$ & - & - & 12 & 1.6 & 6.8 & 576 \\
\hline \multirow[t]{9}{*}{332440090502197} & LC & $\mathrm{HW}$ & $8 / 8 / 2007$ & - & - & 19 & 0.4 & 7.1 & 586 \\
\hline & & & 12/3/2007 & - & - & 0.2 & 0.5 & 6.5 & 683 \\
\hline & & & 2/27/2008 & - & - & 0.1 & 0.3 & 6.9 & 542 \\
\hline & & & 3/6/2008 & - & - & 0.2 & 0.5 & 7.2 & 356 \\
\hline & & & 4/10/2008 & - & - & 0.1 & 0.2 & 7.2 & 420 \\
\hline & & & 6/23/2008 & - & - & 0.1 & 0.2 & 6.8 & 702 \\
\hline & & & 6/25/2008 & -4.22 & - & 3.5 & 0.2 & 6.8 & 670 \\
\hline & & & $8 / 18 / 2008$ & - & - & 0.2 & 0.4 & 6.9 & 680 \\
\hline & & & $11 / 5 / 2008$ & - & - & 0.1 & 0.1 & 6.8 & 671 \\
\hline
\end{tabular}

Table 3. Groundwater-quality data for samples collected at the flowpath along the Bogue Phalia near Leland, MS, 2006-2008 -Continued

[NGVD 29, National Geodetic Vertical Datum of 1929; mg/L, milligram per liter; $\mu \mathrm{g} / \mathrm{L}$, microgram per liter, site type: HW—-hyporheic zone,

\begin{tabular}{|c|c|c|c|c|c|c|c|c|c|c|}
\hline $\begin{array}{l}\text { Station } \\
\text { identifier }\end{array}$ & $\begin{array}{c}\text { Sample } \\
\text { date }\end{array}$ & $\begin{array}{l}\text { Water } \\
\text { temperature } \\
\text { (degrees } \\
\text { Celsius) }\end{array}$ & $\begin{array}{c}\text { Calcium } \\
\text { (mgl/L) }\end{array}$ & $\begin{array}{c}\text { Magnesium } \\
(\text { mg/L) }\end{array}$ & $\begin{array}{c}\begin{array}{c}\text { Potassium } \\
\text { (mg/L) }\end{array} \\
\text { (a) }\end{array}$ & $\begin{array}{c}\text { Sodium } \\
(\mathrm{mgg} / \mathrm{L})\end{array}$ & $\begin{array}{c}\text { Alkalinity, } \\
\text { laboratory } \\
\text { (mal/Las } \\
\text { calcium } \\
\text { carbonate) }\end{array}$ & $\begin{array}{c}\text { Alkalinity, } \\
\text { field, } \\
\text { (myll as } \\
\text { calcium } \\
\text { carbonate) }\end{array}$ & $\begin{array}{l}\text { Bicarbonate, } \\
\text { fiedd } \\
\text { (mg/L) }\end{array}$ & $\begin{array}{l}\text { Bromide, } \\
\text { fiftered } \\
\text { (mg/L) }\end{array}$ \\
\hline \multirow[t]{13}{*}{332440090502001} & 12/5/2006 & 16.8 & 108 & 34.0 & 1.26 & 8.95 & 369 & - & - & 0.03 \\
\hline & 2/13/2007 & 16.3 & 71.3 & 21.6 & 0.92 & 5.83 & 260 & - & - & E0.01 \\
\hline & $5 / 15 / 2007$ & 23.0 & 102 & 32.0 & 1.31 & 6.61 & 372 & - & - & 0.02 \\
\hline & 6/26/2007 & 26.5 & 114 & 33.9 & 1.61 & 7.58 & 393 & - & - & 0.03 \\
\hline & 7/18/2007 & 26.4 & 87.2 & 27.0 & 1.44 & 7.81 & 337 & - & - & 0.06 \\
\hline & $8 / 7 / 2007$ & 26.9 & 73.6 & 22.9 & 1.24 & 7.27 & 290 & - & - & 0.03 \\
\hline & $12 / 3 / 2007$ & 16.4 & 71.9 & 23.1 & 1.19 & 5.50 & 279 & - & - & E 0.01 \\
\hline & 12/4/2007 & 17.8 & 102 & 35.0 & 1.66 & 6.95 & 372 & - & - & 0.03 \\
\hline & 2/27/2008 & 14.4 & 92.7 & 28.1 & 1.14 & 6.33 & 301 & - & - & 0.05 \\
\hline & 3/6/2008 & 15.0 & 106 & 32.8 & 1.40 & 7.07 & 386 & 392 & 477 & 0.08 \\
\hline & 4/10/2008 & 18.7 & 93.3 & 28.3 & 1.18 & 6.72 & 364 & - & - & 0.08 \\
\hline & 6/23/2008 & 24.4 & 67.6 & 22.0 & 1.11 & 5.32 & 258 & - & - & E0.01 \\
\hline & 6/23/2008 & 22.3 & 64.4 & 23.6 & 1.38 & 4.88 & 257 & - & - & E0.01 \\
\hline \multirow[t]{6}{*}{332440090502195} & 12/5/2006 & 7.0 & 106 & 28.1 & 0.98 & 10.8 & 330 & - & - & 0.04 \\
\hline & 2/13/2007 & 16.0 & 108 & 29.2 & 0.91 & 10.6 & 349 & - & - & 0.03 \\
\hline & $5 / 15 / 2007$ & 24.1 & 110 & 29.2 & 0.87 & 10.7 & 349 & - & - & 0.04 \\
\hline & 12/5/2007 & 19.9 & 106 & 26.9 & 2.28 & 11.1 & 340 & - & - & 0.04 \\
\hline & 6/25/2008 & 25.6 & 108 & 27.9 & 1.41 & 9.92 & 363 & - & - & 0.04 \\
\hline & $11 / 4 / 2008$ & 20.7 & 111 & 28.4 & 1.70 & 10.3 & 362 & - & - & 0.04 \\
\hline \multirow[t]{4}{*}{332440090502196} & 8/8/2007 & 28.0 & 90.1 & 21.6 & 1.62 & 9.11 & 349 & - & - & 0.03 \\
\hline & $12 / 5 / 2007$ & 17.3 & 100 & 23.5 & 1.22 & 10.1 & 308 & - & - & 0.02 \\
\hline & 6/25/2008 & 27.6 & 64.9 & 15.3 & 1.72 & 6.56 & 240 & - & - & 0.02 \\
\hline & $11 / 4 / 2008$ & 20.9 & 83.7 & 19.6 & 2.77 & 7.98 & 336 & - & - & 0.05 \\
\hline \multirow[t]{9}{*}{332440090502197} & 8/8/2007 & 24.8 & 81.2 & 18.8 & 3.72 & 11.1 & 280 & - & - & 0.03 \\
\hline & 12/3/2007 & 15.3 & 94.5 & 24.3 & 1.64 & 12.6 & 331 & - & - & 0.03 \\
\hline & 2/27/2008 & 10.9 & 75.5 & 19.4 & 1.21 & 10.3 & 238 & - & - & 0.03 \\
\hline & 3/6/2008 & 14.4 & 50.0 & 12.6 & 1.06 & 8.27 & 160 & - & - & 0.02 \\
\hline & 4/10/2008 & 19.4 & 54.1 & 14.5 & 1.12 & 8.35 & 195 & - & - & 0.02 \\
\hline & 6/23/2008 & 27.7 & 96.5 & 24.9 & 1.60 & 10.9 & 318 & - & - & 0.03 \\
\hline & 6/25/2008 & 21.1 & 95.7 & 24.5 & 1.61 & 11.6 & 311 & - & - & 0.02 \\
\hline & 8/18/2008 & 28.9 & 92.2 & 23.5 & 1.69 & 11.2 & 314 & - & - & 0.03 \\
\hline & $11 / 5 / 2008$ & 18.6 & 97.6 & 25.1 & 1.73 & 12.5 & 318 & - & - & Е0.02 \\
\hline
\end{tabular}


Table 3. Groundwater-quality data for samples collected at the flowpath along the Bogue Phalia near Leland, MS, 2006-2008. - Continued

[NGVD 29, National Geodetic Vertical Datum of 1929; mg/L, milligram per liter, $\mu \mathrm{g} / \mathrm{L}$, microgram per liter, site type: HW-hyporheic zone, GW-groundwater; -, no data; E, estimated

\begin{tabular}{|c|c|c|c|c|c|c|c|c|c|}
\hline $\begin{array}{c}\text { Station } \\
\text { identifier }\end{array}$ & $\begin{array}{c}\text { Sample } \\
\text { date }\end{array}$ & $\begin{array}{c}\text { Chloride } \\
\text { (mgl/) }\end{array}$ & $\begin{array}{c}\text { Fluoride } \\
\text { lmgl/ }\end{array}$ & $\begin{array}{c}\text { Silica } \\
\text { (mg/l } \\
\text { assilica } \\
\text { dioxide) }\end{array}$ & $\begin{array}{l}\text { Sulfate } \\
\text { (mglL) }\end{array}$ & $\begin{array}{c}\text { Ammonia, } \\
\text { filtered } \\
\text { (myllas } \\
\text { nitrogen) }\end{array}$ & $\begin{array}{c}\text { Nitrate plus } \\
\text { nitrite, } \\
\text { fitered, } \\
\text { (myl/Las } \\
\text { nitrogen) }\end{array}$ & $\begin{array}{c}\text { Nitrite, } \\
\text { fittered } \\
\text { (mimfl as } \\
\text { nitrogen) }\end{array}$ & $\begin{array}{c}\text { Ortho- } \\
\text { phosphhate, } \\
\text { filtered } \\
\text { Ims/l as } \\
\text { phosphorus) }\end{array}$ \\
\hline \multirow[t]{13}{*}{332440090502001} & $12 / 5 / 2006$ & 3.10 & 0.28 & 24.9 & 43.7 & 0.313 & $<0.06$ & $<0.002$ & 0.052 \\
\hline & 2/13/2007 & 3.64 & 0.30 & 20.1 & 19.9 & 0.496 & $<0.06$ & E0.002 & 0.295 \\
\hline & $5 / 15 / 2007$ & 3.55 & 0.20 & 21.4 & 41.5 & 0.329 & $<0.06$ & E0.002 & 0.245 \\
\hline & 6/26/2007 & 3.91 & 0.24 & 21.9 & 38.4 & 0.674 & $<0.06$ & 0.002 & 0.194 \\
\hline & 7/18/2007 & 3.97 & 0.28 & 23.5 & 13.8 & 0.888 & $<0.06$ & 0.003 & 0.517 \\
\hline & 8/7/2007 & 3.33 & 0.23 & 22.6 & 13.1 & 0.528 & $<0.06$ & 0.002 & 0.344 \\
\hline & 12/3/2007 & 3.54 & 0.23 & 22.9 & 18.3 & 0.297 & $<0.04$ & E0.002 & 0.295 \\
\hline & $12 / 4 / 2007$ & 3.36 & 0.20 & 22.1 & 57.6 & 0.310 & E0.03 & 0.002 & 0.211 \\
\hline & 2/27/2008 & 4.15 & 0.24 & 21.0 & 10.7 & 0.806 & $<0.04$ & 0.003 & 0.418 \\
\hline & $3 / 6 / 2008$ & 4.47 & 0.22 & 21.5 & 14.1 & 0.955 & $<0.04$ & 0.002 & 0.407 \\
\hline & 4/10/2008 & 4.61 & 0.25 & 21.2 & 22.7 & 1.27 & E0.02 & 0.002 & 0.505 \\
\hline & 6/23/2008 & 3.06 & 0.19 & 19.5 & 19.4 & 0.481 & $<0.04$ & 0.002 & 0.491 \\
\hline & 6/23/2008 & 3.03 & 0.16 & 16.3 & 19.6 & 0.204 & $<0.04$ & E0.001 & 0.272 \\
\hline \multirow[t]{6}{*}{332440090502195} & $12 / 5 / 2006$ & 2.56 & 0.23 & 35.1 & 58.1 & 0.161 & $<0.06$ & $<0.002$ & 0.111 \\
\hline & 2/13/2007 & 2.57 & 0.23 & 31.7 & 56.5 & 0.196 & $<0.06$ & 0.003 & 0.178 \\
\hline & $5 / 15 / 2007$ & 2.64 & 0.23 & 33.9 & 69.3 & 0.162 & $<0.06$ & 0.002 & 0.131 \\
\hline & $12 / 5 / 2007$ & 2.65 & 0.23 & 32.8 & 57.7 & 0.420 & $<0.04$ & 0.002 & 0.263 \\
\hline & $6 / 25 / 2008$ & 2.66 & 0.23 & 30.7 & 51.3 & 0.384 & $<0.04$ & 0.003 & 0.497 \\
\hline & $11 / 4 / 2008$ & 2.50 & 0.26 & 32.6 & 52.4 & 0.767 & $<0.04$ & 0.003 & 0.495 \\
\hline \multirow[t]{4}{*}{332440090502196} & $8 / 8 / 2007$ & 3.14 & 0.19 & 36.5 & 0.34 & 0.307 & $<0.06$ & 0.003 & 0.457 \\
\hline & $12 / 5 / 2007$ & 2.67 & 0.22 & 35.8 & 60.4 & 0.147 & $<0.04$ & $<0.002$ & 0.108 \\
\hline & $6 / 25 / 2008$ & 3.07 & 0.18 & 32.9 & $<0.18$ & 0.505 & $<0.04$ & $<0.002$ & 0.891 \\
\hline & $11 / 4 / 2008$ & 3.50 & 0.20 & 33.1 & 0.26 & 1.28 & E0.03 & E0.002 & 0.832 \\
\hline \multirow{9}{*}{332440090502197} & $8 / 8 / 2007$ & 3.36 & 0.16 & 27.8 & 36.0 & 0.298 & $<0.06$ & E0.001 & 0.123 \\
\hline & $12 / 3 / 2007$ & 2.82 & 0.16 & 36.7 & 45.9 & 0.131 & $<0.04$ & E0.001 & 0.081 \\
\hline & $2 / 27 / 2008$ & 6.35 & 0.17 & 31.4 & 35.0 & 0.102 & $<0.04$ & E0.001 & 0.082 \\
\hline & $3 / 6 / 2008$ & 7.30 & 0.18 & 29.7 & 20.2 & 0.070 & $<0.04$ & $<0.002$ & 0.023 \\
\hline & $4 / 10 / 2008$ & 6.91 & 0.18 & 31.5 & 22.0 & 0.083 & $<0.04$ & $<0.002$ & 0.019 \\
\hline & 6/23/2008 & 2.76 & 0.13 & 34.9 & 50.5 & 0.120 & $<0.04$ & E0.002 & 0.059 \\
\hline & $6 / 25 / 2008$ & 2.89 & 0.20 & 34.1 & 50.5 & 0.125 & $<0.04$ & $<0.002$ & 0.091 \\
\hline & $8 / 18 / 2008$ & 2.70 & 0.15 & 37.9 & 50.0 & 0.127 & $<0.04$ & $<0.002$ & 0.074 \\
\hline & $11 / 5 / 2008$ & 2.51 & 0.17 & 37.5 & 49.9 & 0.124 & $<0.04$ & E0.002 & 0.108 \\
\hline
\end{tabular}

Table 3. Groundwater-quality data for samples collected at the flowpath along the Bogue Phalia near Leland, MS, 2006-2008. - Continued

(a)

\begin{tabular}{|c|c|c|c|c|c|c|c|c|}
\hline $\begin{array}{c}\text { Station } \\
\text { identifier }\end{array}$ & $\begin{array}{c}\text { Sample } \\
\text { date }\end{array}$ & $\begin{array}{l}\text { Phosphorus, } \\
\text { unfiteredd } \\
\text { (mg/Las } \\
\text { phosphorus) }\end{array}$ & $\begin{array}{l}\text { Organic } \\
\text { carbon, } \\
\text { fittered } \\
\text { (mg/L/) }\end{array}$ & $\begin{array}{l}\text { Iron, } \\
\text { fithered } \\
\text { (ug/L) }\end{array}$ & $\begin{array}{l}\text { Manganese, } \\
\text { filtered, } \\
(\mathrm{pg} / \mathrm{L})\end{array}$ & $\begin{array}{c}\text { Aminomethyl- } \\
\text { phosphonic acaid, } \\
\text { recoverable } \\
(\mathrm{pg} / \mathrm{L})\end{array}$ & $\begin{array}{c}\text { Glufosinate } \\
(\mathrm{pg} / \mathrm{L})\end{array}$ & $\begin{array}{c}\text { Glyphosate } \\
(\text { (yg/L) }\end{array}$ \\
\hline \multirow[t]{13}{*}{332440090502001} & $12 / 5 / 2006$ & - & - & 6,630 & 834 & - & - & - \\
\hline & 2/13/2007 & - & - & 6,900 & 1,020 & $<0.02$ & $<0.02$ & $<0.02$ \\
\hline & $5 / 15 / 2007$ & - & 2.3 & 7,320 & 912 & - & - & - \\
\hline & 6/26/2007 & - & 2.7 & 7,740 & 1,300 & $<0.02$ & $<0.02$ & $<0.02$ \\
\hline & 7/18/2007 & - & - & 10,300 & 1,590 & $<0.02$ & $<0.02$ & $<0.02$ \\
\hline & 8/7/2007 & - & 3.6 & 6,590 & 1,000 & $<0.02$ & $<0.02$ & $<0.02$ \\
\hline & $12 / 3 / 2007$ & - & 2.3 & 5,340 & 714 & $<0.02$ & $<0.02$ & $<0.02$ \\
\hline & $12 / 4 / 2007$ & - & 2.2 & 5,960 & 1,160 & - & - & - \\
\hline & $2 / 27 / 2008$ & - & 3.5 & 10,100 & 1,790 & $<0.02$ & $<0.02$ & $<0.02$ \\
\hline & 3/6/2008 & - & 3.7 & 13,200 & 2,530 & $<0.02$ & $<0.02$ & $<0.02$ \\
\hline & $4 / 10 / 2008$ & 0.51 & 4.0 & 14,300 & 2,820 & $<0.02$ & $<0.02$ & 0.03 \\
\hline & $6 / 23 / 2008$ & 0.52 & 3.0 & 6,870 & 1,100 & $<0.02$ & $<0.02$ & $<0.02$ \\
\hline & $6 / 23 / 2008$ & 0.50 & 3.2 & 2,550 & 871 & 0.08 & $<0.02$ & $<0.02$ \\
\hline \multirow[t]{6}{*}{332440090502195} & $12 / 5 / 2006$ & - & - & 8,280 & 555 & - & - & - \\
\hline & 2/13/2007 & - & - & 10,100 & 678 & 0.03 & $<0.02$ & $<0.02$ \\
\hline & 5/15/2007 & - & 2.0 & 10,300 & 692 & - & - & - \\
\hline & $12 / 5 / 2007$ & - & 1.4 & 9,330 & 724 & - & - & - \\
\hline & 6/25/2008 & - & 1.5 & 12,100 & 887 & 0.32 & $<0.02$ & $<0.02$ \\
\hline & $11 / 4 / 2008$ & - & 1.9 & 12,600 & 1,240 & - & - & - \\
\hline \multirow[t]{4}{*}{332440090502196} & 8/8/2007 & - & 3.6 & 3,950 & 474 & 0.12 & $<0.02$ & 0.10 \\
\hline & $12 / 5 / 2007$ & - & 1.5 & 4,670 & 484 & - & - & - \\
\hline & 6/25/2008 & - & 4.2 & 3,980 & 381 & 0.10 & $<0.02$ & 0.11 \\
\hline & $11 / 4 / 2008$ & - & 6.4 & 6,370 & 708 & - & - & - \\
\hline \multirow{9}{*}{332440090502197} & $8 / 8 / 2007$ & - & 2.5 & 781 & 845 & 1.7 & $<0.02$ & 2.0 \\
\hline & $12 / 3 / 2007$ & - & 1.4 & 5,950 & 408 & $<0.02$ & $<0.02$ & $<0.02$ \\
\hline & $2 / 27 / 2008$ & - & 2.4 & 4,580 & 322 & $<0.02$ & $<0.02$ & $<0.02$ \\
\hline & 3/6/2008 & - & 3.5 & 2,740 & 206 & $<0.02$ & $<0.02$ & $<0.02$ \\
\hline & $4 / 10 / 2008$ & 0.09 & 3.1 & 3,200 & 244 & $<0.02$ & $<0.02$ & $<0.02$ \\
\hline & $6 / 23 / 2008$ & 0.07 & 1.7 & 5,960 & 375 & $<0.02$ & $<0.02$ & $<0.02$ \\
\hline & $6 / 25 / 2008$ & - & 1.5 & 5,810 & 496 & 0.12 & $<0.02$ & $<0.02$ \\
\hline & $8 / 18 / 2008$ & - & 1.7 & 5,420 & 349 & - & - & - \\
\hline & $11 / 5 / 2008$ & - & 1.5 & 5,720 & 365 & - & - & - \\
\hline
\end{tabular}


Table 3. Groundwater-quality data for samples collected at the flowpath along the Bogue Phalia near Leland, MS, 2006-2008. - Continued

\begin{tabular}{|c|c|c|c|c|c|c|c|c|c|}
\hline $\begin{array}{c}\text { Station } \\
\text { identifier }\end{array}$ & $\begin{array}{c}\text { Station } \\
\text { name } \\
\text { (fig. and } \\
\text { table 1) }\end{array}$ & $\begin{array}{c}\text { Site } \\
\text { type }\end{array}$ & $\begin{array}{c}\text { Sample } \\
\text { date }\end{array}$ & $\begin{array}{l}\text { Water level } \\
\text { (feet below } \\
\text { land surface) }\end{array}$ & $\begin{array}{c}\text { Water surface } \\
\text { elevation } \\
\text { (feet above } \\
\text { NGVD 29) }\end{array}$ & $\begin{array}{c}\text { Turbidity, in } \\
\text { Nephlememetric } \\
\text { Turbiditity Units }\end{array}$ & $\begin{array}{c}\text { Dissolved } \\
\text { oxygen } \\
\text { (mg/L) }\end{array}$ & $\mathrm{pH}$ & $\begin{array}{c}\text { Specific } \\
\text { conductance, } \\
\text { Imicrosiemens } \\
\text { per centimeter }\end{array}$ \\
\hline \multirow[t]{4}{*}{332440090502201} & \multirow[t]{4}{*}{ LB } & \multirow[t]{4}{*}{$\mathrm{HW}$} & 8/8/2007 & - & - & 1.0 & 0.6 & 7.2 & 327 \\
\hline & & & $12 / 5 / 2007$ & - & - & 11 & 0.3 & 6.9 & 757 \\
\hline & & & 6/25/2008 & -1.97 & - & 3.6 & 0.5 & 6.9 & 537 \\
\hline & & & $11 / 4 / 2008$ & - & - & 1.4 & 0.7 & 7.2 & 348 \\
\hline \multirow[t]{5}{*}{332443090502301} & \multirow[t]{5}{*}{ FS-1A } & \multirow[t]{5}{*}{ GW } & 3/6/2008 & 19.22 & 95.82 & 4.2 & 0.2 & 7.0 & 790 \\
\hline & & & $4 / 10 / 2008$ & 18.78 & 96.26 & 3.5 & 0.3 & 7.0 & 790 \\
\hline & & & 6/24/2008 & 19.40 & 95.64 & 3.0 & 0.1 & 6.7 & 784 \\
\hline & & & $8 / 18 / 2008$ & 20.12 & 94.92 & 3.0 & 1.6 & 6.8 & 763 \\
\hline & & & $11 / 3 / 2008$ & 20.07 & 94.97 & 8.0 & 0.5 & 6.8 & 790 \\
\hline \multirow[t]{13}{*}{332443090502302} & \multirow[t]{13}{*}{ FS-1B } & \multirow[t]{13}{*}{ GW } & $12 / 4 / 2006$ & 21.25 & 93.80 & 0.4 & 0.5 & 6.7 & 1,120 \\
\hline & & & 2/13/2007 & 20.40 & 94.65 & 0.4 & - & 6.7 & 1,160 \\
\hline & & & $5 / 14 / 2007$ & 19.37 & 95.68 & 1.0 & 0.5 & 6.5 & 1,240 \\
\hline & & & 6/26/2007 & 19.39 & 95.66 & 0.2 & 0.3 & 6.6 & 1,290 \\
\hline & & & $7 / 18 / 2007$ & 18.75 & 96.30 & 0.3 & 0.2 & 6.5 & 1,280 \\
\hline & & & 8/7/2007 & 19.71 & 95.34 & 0.6 & 0.2 & 6.5 & 1,300 \\
\hline & & & 12/4/2007 & 20.05 & 95.00 & 0.5 & 0.5 & 6.4 & 1,250 \\
\hline & & & $2 / 26 / 2008$ & 19.52 & 95.53 & 0.1 & 0.3 & 6.6 & 1,250 \\
\hline & & & 3/5/2008 & 19.08 & 95.97 & 0.1 & 0.3 & 6.6 & 1,250 \\
\hline & & & $4 / 10 / 2008$ & 18.70 & 96.35 & 0.4 & 0.3 & 6.7 & 1,320 \\
\hline & & & 6/24/2008 & 20.28 & 94.77 & 0.1 & 0.2 & 6.4 & 1,080 \\
\hline & & & $8 / 19 / 2008$ & 19.91 & 95.14 & 0.3 & 0.4 & 6.6 & 1,270 \\
\hline & & & $11 / 3 / 2008$ & 19.99 & 95.06 & 0.6 & 0.6 & 6.6 & 1,200 \\
\hline \multirow[t]{5}{*}{332443090502501} & \multirow[t]{5}{*}{ FS-2A } & \multirow[t]{5}{*}{ GW } & 3/5/2008 & 18.95 & 95.72 & 0.4 & 0.2 & 6.8 & 721 \\
\hline & & & $4 / 10 / 2008$ & 18.19 & 96.48 & 0.3 & 0.2 & 7.0 & 704 \\
\hline & & & 6/25/2008 & 19.10 & 95.57 & 1.0 & 1.4 & 6.9 & 775 \\
\hline & & & $8 / 19 / 2008$ & 19.78 & 94.89 & 1.0 & 0.4 & 6.8 & 702 \\
\hline & & & $11 / 3 / 2008$ & 19.85 & 94.82 & 0.6 & 0.7 & 6.8 & 709 \\
\hline \multirow[t]{13}{*}{332443090502502} & \multirow[t]{13}{*}{ FS-2B } & \multirow[t]{13}{*}{ GW } & $12 / 4 / 2006$ & 19.51 & 95.34 & 0.3 & - & 6.9 & 1,100 \\
\hline & & & 2/13/2007 & 18.65 & 96.2 & 7.0 & - & 6.6 & 1,060 \\
\hline & & & 5/14/2007 & 19.17 & 95.68 & 4.0 & 0.2 & 6.5 & 1,100 \\
\hline & & & 6/26/2007 & 19.2 & 95.65 & 0.8 & 0.3 & 6.5 & 1,120 \\
\hline & & & 7/18/2007 & 18.91 & 95.94 & 0.9 & 1.3 & 6.4 & 1,060 \\
\hline & & & 8/7/2007 & 19.43 & 95.42 & 1.0 & 0.2 & 6.6 & 1,090 \\
\hline & & & $12 / 4 / 2007$ & 20.17 & 94.68 & 2.2 & 0.3 & 6.5 & 1,150 \\
\hline & & & $2 / 26 / 2008$ & 19.39 & 95.46 & 0.1 & 0.2 & 6.7 & 1,080 \\
\hline & & & 3/5/2008 & 19.00 & 95.85 & 0.3 & 0.2 & 6.6 & 1,080 \\
\hline & & & 4/10/2008 & 18.62 & 96.23 & 2.0 & 0.3 & 6.7 & 1,050 \\
\hline & & & 6/24/2008 & 19.10 & 95.75 & 0.7 & 0.1 & 6.4 & 1,030 \\
\hline & & & $8 / 19 / 2008$ & 19.53 & 95.32 & 2.0 & 0.5 & 6.6 & 1,130 \\
\hline & & & $11 / 3 / 2008$ & 19.80 & 95.05 & 0.3 & 0.8 & 6.6 & 1,140 \\
\hline
\end{tabular}

Table 3. Groundwater-quality data for samples collected at the flowpath along the Bogue Phalia near Leland, MS, 2006-2008. - Continued

[NGVD 29, National Geodetic Vertical Datum of 1929; mg/L, milligram per liter, $\mu \mathrm{g} / \mathrm{L}$, microgram per liter, site type: HW—hyporheic zone,

\begin{tabular}{|c|c|c|c|c|c|c|c|c|c|c|}
\hline $\begin{array}{l}\text { Station } \\
\text { identifier }\end{array}$ & $\begin{array}{c}\text { Sample } \\
\text { date }\end{array}$ & $\begin{array}{c}\text { Water } \\
\text { temperature } \\
\text { (degrees } \\
\text { Celsius) }\end{array}$ & $\begin{array}{c}\text { Calcium } \\
\text { (mgl/) }\end{array}$ & $\begin{array}{c}\text { Magnesium } \\
\text { (mg/L) }\end{array}$ & $\begin{array}{c}\text { Potassium } \\
\text { (mgl/L) }\end{array}$ & $\begin{array}{c}\text { Sodium } \\
(\mathrm{m} / \mathrm{L} / \mathrm{L}\end{array}$ & $\begin{array}{l}\text { Alkalinity, } \\
\text { laborotory } \\
\text { (myllas } \\
\text { calcium } \\
\text { carbonate) }\end{array}$ & $\begin{array}{l}\text { Alkalinity, } \\
\text { field, } \\
\text { (mgl/ as } \\
\text { calcium } \\
\text { carbonate) }\end{array}$ & $\begin{array}{c}\text { Bicarabonate, } \\
\text { field } \\
\text { (my/L) }\end{array}$ & $\begin{array}{l}\text { Bromide, } \\
\text { fititered } \\
\text { (my/L) }\end{array}$ \\
\hline \multirow[t]{4}{*}{332440090502201} & 8/8/2007 & 23.9 & 40.7 & 11.3 & 1.90 & 8.97 & 156 & - & - & 0.02 \\
\hline & 12/5/2007 & 17.4 & 109 & 29.9 & 1.91 & 14.3 & 338 & - & - & 0.03 \\
\hline & 6/25/2008 & 19.4 & 78.1 & 21.7 & 1.52 & 9.94 & 278 & - & - & 0.02 \\
\hline & $11 / 4 / 2008$ & 21.2 & 46.9 & 12.4 & 1.36 & 9.87 & 172 & - & - & 0.07 \\
\hline \multirow{5}{*}{332443090502301} & 3/6/2008 & 18.7 & - & - & - & - & - & 361 & 440 & - \\
\hline & $4 / 10 / 2008$ & 20.7 & 104 & 34.0 & 1.23 & 9.23 & 363 & - & - & 0.12 \\
\hline & 6/24/2008 & 24.2 & 113 & 35.7 & 1.26 & 9.82 & 366 & - & - & 0.10 \\
\hline & $8 / 18 / 2008$ & 23.2 & 104 & 32.7 & 1.15 & 9.35 & 355 & - & - & 0.08 \\
\hline & $11 / 3 / 2008$ & 20.6 & 112 & 35.0 & 1.23 & 9.80 & 359 & - & - & 0.09 \\
\hline \multirow{13}{*}{332443090502302} & $12 / 4 / 2006$ & 20.2 & 167 & 61.5 & 1.39 & 12.0 & 517 & - & - & $<0.02$ \\
\hline & 2/13/2007 & 19.9 & 160 & 56.3 & 1.28 & 11.3 & 508 & - & - & $<0.02$ \\
\hline & $5 / 14 / 2007$ & 19.7 & 179 & 62.4 & 1.32 & 11.9 & 513 & - & - & 0.06 \\
\hline & 6/26/2007 & 21.7 & 191 & 60.9 & 1.30 & 12.2 & 504 & - & - & 0.05 \\
\hline & 7/18/2007 & 20.5 & 189 & 62.8 & 1.57 & 12.2 & 501 & - & - & 0.05 \\
\hline & 8/7/2007 & 20.2 & 176 & 59.0 & 1.20 & 11.4 & 509 & - & - & 0.05 \\
\hline & $12 / 4 / 2007$ & 20.1 & 182 & 61.0 & 1.26 & 11.2 & 493 & - & - & 0.06 \\
\hline & $2 / 26 / 2008$ & 18.8 & 180 & 58.4 & 1.28 & 10.9 & 397 & - & - & 0.07 \\
\hline & 3/5/2008 & 19.5 & 187 & 58.8 & 1.58 & 11.0 & 473 & 255 & 311 & 0.08 \\
\hline & 4/10/2008 & 19.6 & 193 & 60.9 & 1.70 & 10.9 & 470 & - & - & 0.08 \\
\hline & 6/24/2008 & 25.6 & 177 & 54.2 & 1.84 & 10.6 & 466 & - & - & 0.09 \\
\hline & $8 / 19 / 2008$ & 21.1 & 181 & 60.1 & 1.37 & 10.7 & 460 & - & - & 0.08 \\
\hline & $11 / 3 / 2008$ & 20.6 & 169 & 58.5 & 1.43 & 10.8 & 465 & - & - & 0.09 \\
\hline \multirow[t]{5}{*}{332443090502501} & 3/5/2008 & 19.4 & - & - & - & - & - & 321 & 391 & - \\
\hline & 4/10/2008 & 21.2 & 90.8 & 28.3 & 0.54 & 8.68 & 316 & - & - & 0.12 \\
\hline & 6/25/2008 & 22.9 & 105 & 32.1 & 0.62 & 9.39 & 344 & - & - & 0.06 \\
\hline & $8 / 19 / 2008$ & 25.4 & 95.9 & 29.2 & 0.61 & 9.65 & 317 & - & - & 0.04 \\
\hline & $11 / 3 / 2008$ & 19.7 & 98.7 & 29.4 & 0.57 & 9.34 & 316 & - & - & 0.03 \\
\hline \multirow[t]{13}{*}{332443090502502} & $12 / 4 / 2006$ & 20.6 & 167 & 56.4 & 1.54 & 9.38 & 570 & - & - & $<0.02$ \\
\hline & 2/13/2007 & 19.8 & 148 & 54.8 & 1.09 & 9.04 & 531 & - & - & $<0.02$ \\
\hline & $5 / 14 / 2007$ & 19.9 & 153 & 55.8 & 1.29 & 9.10 & 554 & - & - & E0.03 \\
\hline & 6/26/2007 & 20.5 & 157 & 54.3 & 1.33 & 9.44 & 549 & - & - & 0.02 \\
\hline & 7/18/2007 & 21.8 & 146 & 53.7 & 1.17 & 9.26 & 518 & - & - & 0.05 \\
\hline & 8/7/2007 & 26.7 & 146 & 51.4 & 1.23 & 8.80 & 552 & - & - & 0.03 \\
\hline & $12 / 4 / 2007$ & 20.4 & 157 & 54.2 & 1.31 & 8.87 & 558 & - & - & 0.21 \\
\hline & 2/26/2008 & 18.8 & 151 & 53.3 & 1.22 & 8.84 & 414 & - & - & 0.06 \\
\hline & 3/5/2008 & 19.9 & 146 & 51.6 & 1.14 & 8.52 & 527 & 535 & 650 & 0.04 \\
\hline & 4/10/2008 & 20.2 & 140 & 53.0 & 1.07 & 8.82 & 520 & - & - & 0.05 \\
\hline & 6/24/2008 & 24.8 & 150 & 54.7 & 1.20 & 9.03 & 530 & - & - & 0.05 \\
\hline & $8 / 19 / 2008$ & 25.3 & 152 & 53.9 & 1.31 & 8.85 & 543 & - & - & 0.03 \\
\hline & $11 / 3 / 2008$ & 21.1 & 164 & 57.5 & 1.43 & 9.41 & 541 & - & - & 0.04 \\
\hline
\end{tabular}


Table 3. Groundwater-quality data for samples collected at the flowpath along the Bogue Phalia near Leland, MS, 2006-2008. - Continued

[NGVD 29, National Geodetic Vertical Datum of 1929; mg/L, milligram per liter, $\mu \mathrm{g} / \mathrm{L}$, microgram per liter, site type: HW— hyporheic

\begin{tabular}{|c|c|c|c|c|c|c|c|c|c|}
\hline $\begin{array}{c}\text { Station } \\
\text { identifier }\end{array}$ & $\begin{array}{c}\text { Sample } \\
\text { date }\end{array}$ & $\begin{array}{c}\text { Chloride } \\
\text { (mgl/) }\end{array}$ &  & $\begin{array}{c}\text { Silica } \\
\text { (migl as } \\
\text { silica } \\
\text { dioxide) }\end{array}$ & $\begin{array}{l}\text { Sulfate } \\
(\mathrm{mg} / L)\end{array}$ & $\begin{array}{c}\text { Ammonia, } \\
\text { filtered } \\
\text { (my/L as } \\
\text { nitrogen) }\end{array}$ & $\begin{array}{l}\text { Nitrate plus } \\
\text { nitrite, } \\
\text { filtered, } \\
\text { (mg/L as } \\
\text { nitrogen) } \\
\end{array}$ & $\begin{array}{l}\text { Nitrite, } \\
\text { fittered } \\
\text { (mim/ as } \\
\text { nitrogen) }\end{array}$ & $\begin{array}{c}\text { Ortho- } \\
\text { phosphate, } \\
\text { filtered } \\
\text { (mg// as } \\
\text { phosphorus) }\end{array}$ \\
\hline \multirow{4}{*}{332440090502201} & $8 / 8 / 2007$ & 2.70 & 0.26 & 27.1 & 13.5 & 0.160 & $<0.06$ & E0.001 & 0.155 \\
\hline & $12 / 5 / 2007$ & 3.87 & 0.22 & 31.7 & 79.2 & 0.144 & $<0.04$ & E0.001 & 0.113 \\
\hline & $6 / 25 / 2008$ & 3.88 & 0.24 & 22.8 & 32.7 & 0.128 & $<0.04$ & $<0.002$ & 0.130 \\
\hline & $11 / 4 / 2008$ & 4.88 & 0.24 & 22.7 & 7.78 & 0.125 & $<0.04$ & $<0.002$ & 0.108 \\
\hline \multirow[t]{5}{*}{332443090502301} & $3 / 6 / 2008$ & - & - & - & - & - & - & - & - \\
\hline & $4 / 10 / 2008$ & 3.79 & 0.29 & 33.9 & 75.4 & 0.162 & E0.02 & E0.002 & 0.073 \\
\hline & 6/24/2008 & 3.82 & 0.28 & 32.0 & 75.8 & 0.131 & $<0.04$ & E0.002 & 0.046 \\
\hline & $8 / 18 / 2008$ & 3.63 & 0.29 & 31.9 & 73.8 & 0.110 & $<0.04$ & E0.001 & 0.075 \\
\hline & $11 / 3 / 2008$ & 3.64 & 0.30 & 31.4 & 73.7 & 0.097 & $<0.04$ & $<0.002$ & 0.045 \\
\hline \multirow[t]{13}{*}{332443090502302} & $12 / 4 / 2006$ & 7.50 & 0.24 & 34.1 & 130 & 0.069 & $<0.06$ & 0.002 & 0.094 \\
\hline & 2/13/2007 & 6.58 & 0.26 & 30.7 & 156 & 0.064 & $<0.06$ & E0.002 & 0.064 \\
\hline & $5 / 14 / 2007$ & 6.74 & 0.17 & 29.9 & 204 & 0.069 & $<0.06$ & E0.002 & 0.071 \\
\hline & 6/26/2007 & 6.77 & 0.25 & 29.4 & 242 & 0.075 & $<0.06$ & 0.002 & 0.063 \\
\hline & 7/18/2007 & 6.79 & 0.17 & 28.5 & 231 & 0.070 & $<0.06$ & 0.003 & 0.071 \\
\hline & 8/7/2007 & 6.93 & 0.21 & 28.1 & 237 & 0.077 & $<0.06$ & 0.003 & 0.077 \\
\hline & $12 / 4 / 2007$ & 7.55 & 0.25 & 32.8 & 233 & 0.082 & $<0.04$ & 0.002 & 0.055 \\
\hline & 2/26/2008 & 7.14 & 0.25 & 28.7 & 239 & 0.066 & $<0.04$ & $<0.002$ & 0.015 \\
\hline & $3 / 5 / 2008$ & 7.41 & 0.22 & 28.3 & 247 & 0.065 & $<0.04$ & E0.001 & 0.033 \\
\hline & $4 / 10 / 2008$ & 7.29 & 0.22 & 26.8 & 300 & 0.064 & E0.02 & E0.001 & 0.028 \\
\hline & 6/24/2008 & 7.18 & 0.20 & 25.4 & 212 & 0.067 & $<0.04$ & E0.001 & 0.024 \\
\hline & $8 / 19 / 2008$ & 7.16 & 0.14 & 28.8 & 282 & 0.069 & $<0.04$ & E0.001 & 0.063 \\
\hline & $11 / 3 / 2008$ & 7.15 & 0.26 & 29.8 & 219 & 0.068 & $<0.04$ & E0.001 & 0.043 \\
\hline \multirow[t]{5}{*}{332443090502501} & $3 / 5 / 2008$ & - & - & - & - & - & - & - & - \\
\hline & $4 / 10 / 2008$ & 4.39 & 0.30 & 31.8 & 61.4 & 0.126 & $<0.04$ & $<0.002$ & 0.083 \\
\hline & $6 / 25 / 2008$ & 5.39 & 0.26 & 30.0 & 67.9 & 0.121 & $<0.04$ & E0.002 & 0.184 \\
\hline & $8 / 19 / 2008$ & 4.26 & 0.31 & 29.4 & 61.9 & 0.186 & $<0.04$ & 0.003 & 0.164 \\
\hline & $11 / 3 / 2008$ & 4.15 & 0.33 & 29.4 & 61.6 & 0.153 & $<0.04$ & $<0.002$ & 0.025 \\
\hline \multirow[t]{12}{*}{332443090502502} & $12 / 4 / 2006$ & 8.34 & 0.22 & 35.0 & 66.5 & 0.078 & $<0.06$ & 0.002 & 0.079 \\
\hline & 2/13/2007 & 8.89 & 0.23 & 32.8 & 71.2 & 0.076 & $<0.06$ & E0.001 & 0.048 \\
\hline & $5 / 14 / 2007$ & 8.54 & 0.17 & 31.8 & 69.2 & 0.084 & $<0.06$ & E0.002 & 0.064 \\
\hline & 6/26/2007 & 8.55 & 0.19 & 31.4 & 78.1 & 0.074 & $<0.06$ & E0.001 & 0.050 \\
\hline & 7/18/2007 & 8.93 & 0.18 & 32.7 & 72.5 & 0.083 & $<0.06$ & 0.003 & 0.075 \\
\hline & $8 / 7 / 2007$ & 8.89 & 0.21 & 30.4 & 72.0 & 0.077 & $<0.06$ & 0.002 & 0.080 \\
\hline & $12 / 4 / 2007$ & 10.4 & 0.23 & 33.2 & 90.8 & 0.087 & $<0.04$ & 0.002 & 0.066 \\
\hline & 2/26/2008 & 9.24 & 0.25 & 32.4 & 75.5 & 0.09 & $<0.04$ & E0.002 & 0.064 \\
\hline & $3 / 5 / 2008$ & 9.27 & 0.22 & 32.2 & 75.9 & 0.081 & $<0.04$ & E0.001 & 0.032 \\
\hline & 4/10/2008 & 10.4 & 0.22 & 33.8 & 75.4 & 0.068 & E0.02 & $<0.002$ & 0.014 \\
\hline & 6/24/2008 & 10.3 & 0.18 & 30.6 & 74.0 & 0.085 & $<0.04$ & E0.001 & 0.071 \\
\hline & 8/19/2008 & 10.1 & 0.14 & 31.2 & 97.2 & 0.085 & $<0.04$ & E0.001 & 0.096 \\
\hline
\end{tabular}

Table 3. Groundwater-quality data for samples collected at the flowpath along the Bogue Phalia near Leland, MS, 2006-2008. -Continued

作

\begin{tabular}{|c|c|c|c|c|c|c|c|c|}
\hline $\begin{array}{l}\text { Station } \\
\text { identifier }\end{array}$ & $\begin{array}{c}\text { Sample } \\
\text { date }\end{array}$ & $\begin{array}{l}\text { Phosphorus, } \\
\text { unfiltered } \\
\text { (mg/L as } \\
\text { phosphorus) }\end{array}$ & $\begin{array}{l}\text { Organic } \\
\text { carbon, } \\
\text { fitiered } \\
\text { (mgl/L) }\end{array}$ & $\begin{array}{l}\text { Iron, } \\
\text { fititered } \\
(\text { (ug/L) }\end{array}$ & $\begin{array}{c}\text { Manganese, } \\
\text { fititered } \\
\text { (tyg/L) }\end{array}$ & $\begin{array}{c}\text { Aminomethyl- } \\
\text { phosphonic acid, } \\
\text { recoverable } \\
\text { (gg/L) }\end{array}$ & $\begin{array}{l}\text { Glufosinate } \\
\text { (lyg/L) }\end{array}$ & $\begin{array}{c}\text { Giyphosate } \\
(\mathbf{p g / L / L})\end{array}$ \\
\hline \multirow[t]{4}{*}{332440090502201} & $8 / 8 / 2007$ & - & 3.5 & 994 & 334 & 0.54 & $<0.02$ & 0.54 \\
\hline & $12 / 5 / 2007$ & - & 1.3 & 3,760 & 606 & - & - & - \\
\hline & 6/25/2008 & - & 2.1 & 2,450 & 421 & 0.14 & $<0.02$ & 0.10 \\
\hline & $11 / 4 / 2008$ & - & 3.8 & 1,180 & 264 & - & - & - \\
\hline \multirow[t]{5}{*}{332443090502301} & 3/6/2008 & - & - & - & - & - & - & - \\
\hline & $4 / 10 / 2008$ & 0.22 & 2.1 & 6,900 & 592 & - & - & - \\
\hline & 6/24/2008 & 0.41 & 1.9 & 7,400 & 554 & - & - & - \\
\hline & $8 / 18 / 2008$ & - & 1.8 & 7,170 & 549 & - & - & - \\
\hline & $11 / 3 / 2008$ & - & 1.3 & 7,330 & 605 & - & - & - \\
\hline \multirow[t]{13}{*}{332443090502302} & $12 / 4 / 2006$ & - & - & 7,520 & 956 & - & - & - \\
\hline & $2 / 13 / 2007$ & - & - & 6,260 & 1,040 & $<0.02$ & $<0.02$ & $<0.02$ \\
\hline & $5 / 14 / 2007$ & - & 2.2 & 9,800 & 926 & - & - & - \\
\hline & 6/26/2007 & - & 2.0 & 9,290 & 953 & $<0.02$ & $<0.02$ & $<0.02$ \\
\hline & 7/18/2007 & - & - & 8,220 & 994 & $<0.02$ & $<0.02$ & $<0.02$ \\
\hline & 8/7/2007 & - & 2.1 & 9,640 & 894 & - & - & - \\
\hline & $12 / 4 / 2007$ & - & 2.1 & 9,080 & 943 & - & - & - \\
\hline & 2/26/2008 & - & 2.3 & 8,870 & 901 & - & - & - \\
\hline & 3/5/2008 & - & 2.1 & 8,200 & 1,010 & - & - & - \\
\hline & $4 / 10 / 2008$ & E0.01 & 2.1 & 6,680 & 1,130 & - & - & - \\
\hline & 6/24/2008 & 0.04 & 2.2 & 6,880 & 993 & - & - & - \\
\hline & $8 / 19 / 2008$ & - & 2.0 & 9,650 & 931 & - & - & - \\
\hline & $11 / 3 / 2008$ & - & 1.6 & 8,410 & 976 & - & - & - \\
\hline \multirow[t]{5}{*}{332443090502501} & 3/5/2008 & - & - & - & - & - & - & - \\
\hline & $4 / 10 / 2008$ & 0.18 & 2.2 & 7,910 & 675 & - & - & - \\
\hline & $6 / 25 / 2008$ & - & 1.6 & 9,020 & 691 & - & - & - \\
\hline & $8 / 19 / 2008$ & - & 2.0 & 7,860 & 706 & - & - & - \\
\hline & $11 / 3 / 2008$ & - & 1.3 & 7,940 & 723 & - & - & - \\
\hline \multirow[t]{13}{*}{332443090502502} & $12 / 4 / 2006$ & - & - & 7,680 & 708 & - & - & - \\
\hline & $2 / 13 / 2007$ & - & - & 8,460 & 598 & $<0.02$ & $<0.02$ & $<0.02$ \\
\hline & $5 / 14 / 2007$ & - & 2.3 & 7,640 & 665 & - & - & - \\
\hline & 6/26/2007 & - & 1.8 & 7,650 & 653 & $<0.02$ & $<0.02$ & $<0.02$ \\
\hline & 7/18/2007 & - & - & 8,610 & 576 & $<0.02$ & $<0.02$ & $<0.02$ \\
\hline & 8/7/2007 & - & 2.0 & 7,920 & 602 & - & - & - \\
\hline & $12 / 4 / 2007$ & - & 2.0 & 8,350 & 595 & - & - & - \\
\hline & 2/26/2008 & - & 2.5 & 8,500 & 584 & - & - & - \\
\hline & 3/5/2008 & - & 2.4 & 8,810 & 586 & - & - & - \\
\hline & $4 / 10 / 2008$ & 0.12 & 2.3 & 8,480 & 540 & - & - & - \\
\hline & 6/24/2008 & 0.13 & 2.0 & 8,280 & 545 & - & - & - \\
\hline & $8 / 19 / 2008$ & - & 1.9 & 8,740 & 554 & - & - & - \\
\hline & $11 / 3 / 2008$ & - & 2.1 & 8,310 & 632 & - & - & - \\
\hline
\end{tabular}


Table 3. Groundwater-quality data for samples collected at the flowpath along the Bogue Phalia near Leland, MS, 2006-2008. - Continued

[NGVD 29, National Geodetic Vertical Datum of 1929; mg/L, milligram per liter; $\mu \mathrm{g} / \mathrm{L}$, microgram per liter, site type: HW-hyporheic zone,

\begin{tabular}{|c|c|c|c|c|c|c|c|c|c|}
\hline $\begin{array}{l}\text { Station } \\
\text { identifier }\end{array}$ & $\begin{array}{c}\text { Station } \\
\text { name } \\
\text { (fig. 1 and } \\
\text { table 1) }\end{array}$ & $\begin{array}{c}\text { Site } \\
\text { type }\end{array}$ & $\begin{array}{c}\text { Sample } \\
\text { date }\end{array}$ & $\begin{array}{l}\text { Water level } \\
\text { (fieet below } \\
\text { land surface) }\end{array}$ & $\begin{array}{l}\text { Water- surface } \\
\text { elevation } \\
\text { (feet above } \\
\text { NGVD 29) }\end{array}$ & $\begin{array}{c}\text { Turbidity, in } \\
\text { Nephlememertic } \\
\text { Turbiditity Units }\end{array}$ & $\begin{array}{c}\text { Dissolved } \\
\text { oxygen } \\
\text { (mgl/L) }\end{array}$ & $\mathrm{pH}$ & $\begin{array}{c}\text { Specific } \\
\text { conductance, } \\
\text { (mirrosiement } \\
\text { per centimeter }\end{array}$ \\
\hline \multirow[t]{4}{*}{332443090502701} & FS-3A & $\mathrm{GW}$ & $3 / 3 / 2008$ & 18.92 & 95.7 & 0.6 & 0.2 & 6.7 & 1,090 \\
\hline & & & 6/24/2008 & 18.80 & 95.82 & 0.5 & 0.4 & 6.6 & 1,080 \\
\hline & & & $8 / 19 / 2008$ & 19.45 & 95.17 & 0.7 & 3.6 & 6.6 & 1,040 \\
\hline & & & $11 / 3 / 2008$ & 19.55 & 95.07 & 2.1 & 0.5 & 6.9 & 1,040 \\
\hline \multirow[t]{12}{*}{332443090502702} & FS-3B & $\mathrm{GW}$ & $12 / 4 / 2006$ & 18.21 & 96.23 & 1.0 & - & 6.5 & 1,150 \\
\hline & & & 2/13/2007 & 18.40 & 96.04 & 1.2 & - & 6.6 & 1,170 \\
\hline & & & $5 / 15 / 2007$ & 18.91 & 95.53 & 2.0 & 0.3 & 6.4 & 969 \\
\hline & & & 6/26/2007 & 18.95 & 95.49 & 0.3 & 0.3 & 6.5 & 1,140 \\
\hline & & & 7/18/2007 & 18.37 & 96.07 & 0.8 & 0.2 & 6.4 & 1,100 \\
\hline & & & 8/7/2007 & 19.13 & 95.31 & 0.6 & 0.2 & 6.4 & 1,160 \\
\hline & & & $12 / 5 / 2007$ & 19.64 & 94.8 & 0.8 & 0.3 & 6.2 & 1,010 \\
\hline & & & 2/26/2008 & 19.12 & 95.32 & 0.1 & 0.2 & 6.7 & 916 \\
\hline & & & $3 / 3 / 2008$ & 18.78 & 95.66 & 0.3 & 0.4 & 6.6 & 941 \\
\hline & & & 6/24/2008 & 18.80 & 95.64 & 0.9 & 0.2 & 6.4 & 1,040 \\
\hline & & & $8 / 19 / 2008$ & 19.38 & 95.06 & 0.3 & 0.5 & 6.4 & 1,050 \\
\hline & & & $11 / 3 / 2008$ & 19.50 & 94.94 & 0.2 & 0.5 & 6.6 & 908 \\
\hline \multirow[t]{13}{*}{332445090501601} & AR-1A & $\mathrm{GW}$ & $12 / 5 / 2006$ & 17.37 & 95.32 & 0.5 & - & 7.2 & 598 \\
\hline & & & 2/13/2007 & 16.67 & 96.02 & 0.8 & - & 7.2 & 789 \\
\hline & & & 5/15/2007 & 17.27 & 95.42 & 0.7 & 0.3 & 6.9 & 931 \\
\hline & & & 6/26/2007 & 17.25 & 95.44 & 0.1 & 0.4 & 6.8 & 1,180 \\
\hline & & & 7/18/2007 & 16.68 & 96.01 & 0.6 & 0.2 & 6.8 & 519 \\
\hline & & & 8/7/2007 & 17.36 & 95.33 & 0.9 & 0.2 & 7.1 & 459 \\
\hline & & & $12 / 5 / 2007$ & 17.97 & 94.72 & 0.3 & 0.3 & 6.8 & 614 \\
\hline & & & 2/26/2008 & 17.50 & 95.19 & 0.1 & 0.3 & 7.0 & 612 \\
\hline & & & $3 / 3 / 2008$ & 17.33 & 95.36 & 0.2 & 0.3 & 7.0 & 597 \\
\hline & & & 4/9/2008 & 16.56 & 96.13 & 0.2 & 0.4 & 7.0 & 1,100 \\
\hline & & & 6/23/2008 & 17.15 & 95.54 & 0.3 & 0.2 & 6.9 & 940 \\
\hline & & & $8 / 18 / 2008$ & 17.77 & 94.92 & 0.2 & 0.3 & 6.9 & 751 \\
\hline & & & $11 / 3 / 2008$ & 17.91 & 94.78 & 0.1 & 0.5 & 6.7 & 980 \\
\hline
\end{tabular}

Table 3. Groundwater-quality data for samples collected at the flowpath along the Bogue Phalia near Leland, MS, 2006-2008. - Continued

[NGVD 29, National Geodetic Vertical Datum of 1929; mg/L, milligram per liter, $\mu \mathrm{g} / \mathrm{L}$, microgram per liter, site type: HW—hyporheic zone,

\begin{tabular}{|c|c|c|c|c|c|c|c|c|c|c|}
\hline $\begin{array}{c}\text { Station } \\
\text { identifier }\end{array}$ & $\begin{array}{c}\text { Sample } \\
\text { date }\end{array}$ & $\begin{array}{c}\text { Water } \\
\text { temperature } \\
\text { (degrees } \\
\text { Celsius) }\end{array}$ & $\begin{array}{c}\text { Calcium } \\
\text { (mg/L) }\end{array}$ & $\underset{\substack{\text { Magnesium } \\
(\mathrm{mg} / \mathrm{L})}}{ }$ & $\begin{array}{c}\text { Potassium } \\
\text { (mg/L/L) }\end{array}$ & $\begin{array}{c}\text { Sodium } \\
\text { (mg/L) }\end{array}$ & $\begin{array}{l}\text { Alkalinity, } \\
\text { laboratory } \\
\text { (Igy/las as } \\
\text { calcium } \\
\text { carbonate) }\end{array}$ & $\begin{array}{c}\text { Alkalinity, } \\
\text { field, } \\
\text { (myltas } \\
\text { calcium } \\
\text { carbonate) }\end{array}$ & $\begin{array}{c}\text { Bicarbonate, } \\
\text { field } \\
(\mathrm{m} / \mathrm{L})\end{array}$ & $\begin{array}{c}\text { Bromide, } \\
\text { fituterd } \\
\text { (mgg/l) }\end{array}$ \\
\hline \multirow[t]{4}{*}{332443090502701} & $3 / 3 / 2008$ & 20.2 & - & - & - & - & - & - & - & - \\
\hline & 6/24/2008 & 26.6 & 152 & 48.6 & 0.71 & 11.6 & 533 & - & - & 0.30 \\
\hline & 8/19/2008 & 22.3 & 142 & 46.3 & 0.71 & 12.2 & 490 & - & - & 0.25 \\
\hline & $11 / 3 / 2008$ & 22.7 & 150 & 47.2 & 0.72 & 12.1 & 497 & - & - & 0.24 \\
\hline \multirow[t]{12}{*}{332443090502702} & $12 / 4 / 2006$ & 20.1 & 163 & 45.4 & 1.69 & 13.6 & 516 & - & - & $<0.02$ \\
\hline & 2/13/2007 & 17.3 & 179 & 51.0 & 1.80 & 14.2 & 557 & - & - & $<0.02$ \\
\hline & $5 / 15 / 2007$ & 19.5 & 140 & 38.6 & 1.47 & 12.3 & 441 & - & - & 0.16 \\
\hline & 6/26/2007 & 20.2 & 169 & 45.8 & 1.67 & 14.2 & 472 & - & - & 0.16 \\
\hline & $7 / 18 / 2007$ & 20.3 & 166 & 45.8 & 1.74 & 13.9 & 468 & - & - & 0.16 \\
\hline & 8/7/2007 & 20.0 & 172 & 45.8 & 1.61 & 14.2 & 482 & - & - & 0.16 \\
\hline & $12 / 5 / 2007$ & 19.3 & 148 & 39.9 & 1.61 & 14.3 & 456 & - & - & 0.17 \\
\hline & $2 / 26 / 2008$ & 18.2 & 131 & 36.1 & 1.42 & 13.7 & 298 & - & - & 0.15 \\
\hline & $3 / 3 / 2008$ & 20.0 & 136 & 37.8 & 1.46 & 13.8 & 413 & 421 & 512 & 0.15 \\
\hline & 6/24/2008 & 23.9 & 154 & 43.2 & 1.71 & 13.6 & 438 & - & - & 0.15 \\
\hline & 8/19/2008 & 20.2 & 147 & 40.9 & 1.56 & 13.9 & 445 & - & - & 0.15 \\
\hline & $11 / 3 / 2008$ & 22.1 & 135 & 37.2 & 1.60 & 13.4 & 414 & - & - & 0.17 \\
\hline \multirow[t]{13}{*}{332445090501601} & $12 / 5 / 2006$ & 18.3 & 92.4 & 22.5 & 1.39 & 10.6 & 194 & - & - & E0.01 \\
\hline & 2/13/2007 & 16.3 & 117 & 30.1 & 1.30 & 9.89 & 299 & - & - & E0.02 \\
\hline & $5 / 15 / 2007$ & 17.8 & 145 & 36.9 & 1.28 & 9.88 & 290 & - & - & E0.02 \\
\hline & 6/26/2007 & 18.6 & 182 & 50.6 & 1.35 & 14.7 & 311 & - & - & E0.01 \\
\hline & 7/18/2007 & 17.9 & 71.5 & 20.3 & 0.92 & 7.50 & 221 & - & - & E0.02 \\
\hline & $8 / 7 / 2007$ & 18.0 & 62.8 & 17.5 & 0.78 & 6.55 & 198 & - & - & $<0.02$ \\
\hline & $12 / 5 / 2007$ & 18.5 & 85.8 & 23.0 & 0.92 & 7.78 & 220 & - & - & E0.01 \\
\hline & 2/26/2008 & 17.5 & 88.6 & 24.7 & 0.88 & 5.42 & 262 & - & - & E0.02 \\
\hline & $3 / 3 / 2008$ & 18.4 & 85 & 23.8 & 0.87 & 5.18 & 288 & 281 & 338 & E0.02 \\
\hline & 4/9/2008 & 18.2 & 171 & 43.9 & 1.22 & 7.19 & 354 & - & - & E0.01 \\
\hline & $6 / 23 / 2008$ & 19.1 & 108 & 53.0 & 1.25 & 10.4 & 364 & - & - & E0.01 \\
\hline & $8 / 18 / 2008$ & 18.9 & 88.3 & 41.5 & 1.02 & 6.04 & 277 & - & - & 0.03 \\
\hline & $11 / 3 / 2008$ & 18.7 & 131 & 52.8 & 1.14 & 7.48 & 364 & - & - & 0.01 \\
\hline
\end{tabular}


Table 3. Groundwater-quality data for samples collected at the flowpath along the Bogue Phalia near Leland, MS, 2006-2008. - Continued

NGVD 29, National Geodetic Vertical Datum of 1929; mg/L, milligram per liter; $\mu \mathrm{g} / \mathrm{L}$, microgram per liter, site type: HW- hyporheic zoo GW-groundwater; - - no data; $\mathrm{E}$, estimated]

\begin{tabular}{|c|c|c|c|c|c|c|c|c|c|}
\hline $\begin{array}{c}\text { Station } \\
\text { identifier }\end{array}$ & $\begin{array}{c}\begin{array}{c}\text { Sample } \\
\text { date }\end{array} \\
\text { a }\end{array}$ & $\begin{array}{c}\text { Chloride } \\
(\mathrm{mg} / \mathrm{L})\end{array}$ & $\begin{array}{c}\text { Fluoride } \\
\text { (mg/L }\end{array}$ & $\begin{array}{c}\text { Silica } \\
\text { (mygl as } \\
\text { silica } \\
\text { dioxide) }\end{array}$ & $\begin{array}{c}\text { Sulfate } \\
\text { (mgl/L) }\end{array}$ & $\begin{array}{c}\text { Ammonia, } \\
\text { fittered } \\
\text { (mygl/ as } \\
\text { nitrogen) } \\
\end{array}$ & $\begin{array}{l}\text { Nitrate plus } \\
\text { nitrite, } \\
\text { fitered, } \\
\text { (mygl/Las } \\
\text { nitrogen) }\end{array}$ & $\begin{array}{l}\text { Nitrite, } \\
\text { fittered } \\
\text { (mygl/ as } \\
\text { nitrogen) } \\
\end{array}$ & $\begin{array}{l}\text { Ortho- } \\
\text { phosphate, } \\
\text { fitered } \\
\text { (Itg/L as } \\
\text { phosphorus) }\end{array}$ \\
\hline \multirow[t]{4}{*}{332443090502701} & $3 / 3 / 2008$ & - & - & - & - & - & - & - & - \\
\hline & 6/24/2008 & 19.3 & 0.23 & 30.6 & 60.0 & 0.186 & E0.02 & E0.002 & 0.198 \\
\hline & $8 / 19 / 2008$ & 16.1 & 0.25 & 29.5 & 68.5 & 0.160 & $<0.04$ & 0.003 & 0.103 \\
\hline & $11 / 3 / 2008$ & 16.8 & 0.27 & 30.8 & 76.0 & 0.178 & $<0.04$ & E0.001 & 0.072 \\
\hline \multirow[t]{12}{*}{332443090502702} & $12 / 4 / 2006$ & 18.6 & 0.22 & 28.1 & 62.7 & 0.073 & $<0.06$ & Е0.002 & 0.098 \\
\hline & 2/13/2007 & 19.2 & 0.19 & 25.8 & 99.6 & 0.063 & $<0.06$ & E0.001 & 0.062 \\
\hline & $5 / 15 / 2007$ & 13.4 & 0.15 & 25.6 & 97.0 & 0.056 & $<0.06$ & Е0.001 & 0.073 \\
\hline & 6/26/2007 & 16.1 & 0.25 & 25.2 & 146 & 0.072 & $<0.06$ & 0.003 & 0.067 \\
\hline & 7/18/2007 & 16.0 & 0.16 & 25.1 & 137 & 0.066 & $<0.06$ & 0.002 & 0.059 \\
\hline & $8 / 7 / 2007$ & 16.9 & 0.17 & 24.9 & 146 & 0.066 & $<0.06$ & Е0.002 & 0.073 \\
\hline & $12 / 5 / 2007$ & 14.0 & 0.25 & 26.1 & 96.5 & 0.085 & $<0.04$ & Е0.001 & 0.054 \\
\hline & 2/26/2008 & 10.2 & 0.21 & 25.0 & 84.1 & 0.076 & $<0.04$ & E0.001 & 0.072 \\
\hline & $3 / 3 / 2008$ & 10.5 & 0.19 & 24.6 & 101 & 0.069 & $<0.04$ & E0.001 & 0.064 \\
\hline & 6/24/2008 & 10.2 & 0.18 & 22.9 & 153 & 0.064 & $<0.04$ & Е0.001 & 0.065 \\
\hline & $8 / 19 / 2008$ & 11.5 & 0.14 & 24.4 & 146 & 0.057 & $<0.04$ & $<0.002$ & 0.024 \\
\hline & $11 / 3 / 2008$ & 9.89 & 0.22 & 25.1 & 90.2 & 0.051 & $<0.04$ & $<0.002$ & 0.017 \\
\hline \multirow[t]{13}{*}{332445090501601} & $12 / 5 / 2006$ & 4.63 & 0.38 & 20.1 & 123 & E0.017 & $<0.06$ & $<0.002$ & 0.071 \\
\hline & 2/13/2007 & 5.85 & 0.52 & 17.1 & 124 & Е0.018 & $<0.06$ & $<0.002$ & 0.097 \\
\hline & $5 / 15 / 2007$ & 5.98 & 0.42 & 18.1 & 236 & E0.016 & $<0.06$ & Е0.001 & 0.090 \\
\hline & 6/26/2007 & 7.60 & 0.42 & 19.3 & 358 & E0.018 & $<0.06$ & $<0.002$ & 0.016 \\
\hline & $7 / 18 / 2007$ & 2.53 & 0.63 & 15.7 & 53.0 & $<0.020$ & $<0.06$ & Е0.001 & 0.014 \\
\hline & $8 / 7 / 2007$ & 2.16 & 0.64 & 15.5 & 44.3 & E0.014 & $<0.06$ & Е0.001 & 0.095 \\
\hline & $12 / 5 / 2007$ & 3.93 & 0.58 & 17.3 & 101 & E0.014 & $<0.04$ & $<0.002$ & 0.085 \\
\hline & 2/26/2008 & 3.21 & 0.56 & 16.1 & 40.7 & Е0.017 & $<0.04$ & $<0.002$ & 0.068 \\
\hline & $3 / 3 / 2008$ & 3.36 & 0.54 & 15.9 & 36.5 & E0.011 & $<0.04$ & $<0.002$ & 0.101 \\
\hline & $4 / 9 / 2008$ & 11.4 & 0.40 & 16.3 & 273 & E0.018 & $<0.04$ & $<0.002$ & 0.059 \\
\hline & $6 / 23 / 2008$ & 9.41 & 0.31 & 16.8 & 142 & 0.023 & $<0.04$ & $<0.002$ & 0.074 \\
\hline & $8 / 18 / 2008$ & 6.14 & 0.33 & 15.2 & 137 & 0.109 & $<0.04$ & $<0.002$ & 0.082 \\
\hline & $11 / 3 / 2008$ & 5.58 & 0.29 & 15.6 & 188 & 0.038 & $<0.04$ & $<0.002$ & 0.035 \\
\hline
\end{tabular}

Table 3. Groundwater-quality data for samples collected at the flowpath along the Bogue Phalia near Leland, MS, 2006-2008. - Continued

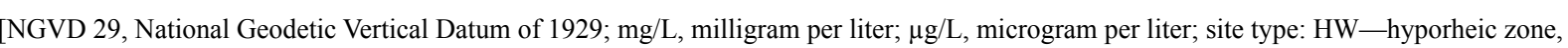

\begin{tabular}{|c|c|c|c|c|c|c|c|c|}
\hline $\begin{array}{l}\text { Station } \\
\text { identifier }\end{array}$ & $\begin{array}{c}\text { Sample } \\
\text { date }\end{array}$ & $\begin{array}{l}\text { Phosphorus, } \\
\text { unfiltered } \\
\text { (mg// as } \\
\text { phosphorus) }\end{array}$ & $\begin{array}{l}\text { Organic } \\
\text { carbon, } \\
\text { fitrered } \\
\text { (my/L) }\end{array}$ & $\begin{array}{l}\text { Iron, } \\
\text { fititered } \\
(\mathrm{pg} / L)\end{array}$ & $\begin{array}{l}\text { Manganese, } \\
\text { fititerd } \\
\text { (ug/L) }\end{array}$ & $\begin{array}{l}\text { Aminomethyl- } \\
\text { phosphonic acid, } \\
\text { recoverable } \\
\text { (yg/L) }\end{array}$ & $\begin{array}{l}\text { Glufosinate } \\
(\mathrm{lyg} / \mathrm{L})\end{array}$ & $\begin{array}{c}\text { Glyphosate } \\
(\lg / / L)\end{array}$ \\
\hline \multirow[t]{4}{*}{332443090502701} & $3 / 3 / 2008$ & - & - & - & - & - & - & - \\
\hline & 6/24/2008 & 0.17 & 2.4 & 15,200 & 1,140 & - & - & - \\
\hline & $8 / 19 / 2008$ & - & 2.0 & 13,900 & 1,140 & - & - & - \\
\hline & $11 / 3 / 2008$ & - & 1.8 & 14,200 & 1,220 & - & - & - \\
\hline \multirow[t]{12}{*}{332443090502702} & $12 / 4 / 2006$ & - & - & 7,070 & 1,670 & - & - & - \\
\hline & 2/13/2007 & - & - & 6,020 & 1,990 & $<0.02$ & $<0.02$ & $<0.02$ \\
\hline & $5 / 15 / 2007$ & - & 2.2 & 4,950 & 1,730 & - & - & - \\
\hline & 6/26/2007 & - & 2.0 & 6,580 & 1,930 & $<0.02$ & $<0.02$ & $<0.02$ \\
\hline & 7/18/2007 & - & - & 5,720 & 1,890 & $<0.02$ & $<0.02$ & $<0.02$ \\
\hline & $8 / 7 / 2007$ & - & 2.3 & 6,780 & 1,820 & - & - & - \\
\hline & $12 / 5 / 2007$ & - & 2.5 & 6,020 & 1,560 & - & - & - \\
\hline & 2/26/2008 & - & 2.3 & 5,470 & 1,370 & - & - & - \\
\hline & $3 / 3 / 2008$ & - & 2.4 & 5,710 & 1,460 & - & - & - \\
\hline & 6/24/2008 & 0.06 & 2.3 & 4,980 & 1,730 & - & - & - \\
\hline & $8 / 19 / 2008$ & - & 2.1 & 6,170 & 1,580 & - & - & - \\
\hline & $11 / 3 / 2008$ & - & 1.7 & 5,130 & 1,530 & - & - & - \\
\hline \multirow[t]{13}{*}{332445090501601} & $12 / 5 / 2006$ & - & - & 2,400 & 248 & - & - & - \\
\hline & 2/13/2007 & - & - & 2,760 & 385 & $<0.02$ & $<0.02$ & $<0.02$ \\
\hline & $5 / 15 / 2007$ & - & 2.6 & 3,510 & 523 & - & - & - \\
\hline & 6/26/2007 & - & 2.3 & 4,830 & 793 & $<0.02$ & $<0.02$ & $<0.02$ \\
\hline & 7/18/2007 & - & - & 1,550 & 351 & $<0.02$ & $<0.02$ & $<0.02$ \\
\hline & $8 / 7 / 2007$ & - & 2.6 & 1,360 & 306 & - & - & - \\
\hline & $12 / 5 / 2007$ & - & 2.4 & 1,860 & 428 & - & - & - \\
\hline & 2/26/2008 & - & 2.6 & 1,920 & 416 & $<0.02$ & $<0.02$ & $<0.02$ \\
\hline & $3 / 3 / 2008$ & - & 2.5 & 1,780 & 411 & $<0.02$ & $<0.02$ & $<0.02$ \\
\hline & 4/9/2008 & 0.06 & 3.1 & 3,610 & 784 & $<0.02$ & $<0.02$ & $<0.02$ \\
\hline & 6/23/2008 & 0.09 & 2.9 & 35,30 & 899 & - & - & - \\
\hline & $8 / 18 / 2008$ & - & 2.8 & 2,430 & 701 & - & - & - \\
\hline & $11 / 3 / 2008$ & & 2.5 & 3,920 & 1,220 & - & - & - \\
\hline
\end{tabular}


Table 4. Surface-water-quality data from samples collected at station 07288636, Tommie Bayou at Pace, MS. $\left[\mathrm{mg} / \mathrm{L}\right.$, milligram per liter; $\mathrm{SiO}_{2}$, silica dioxide; $\mu \mathrm{g} / \mathrm{L}$, microgram per liter; $\mathrm{UV}$, ultraviolet; $\mathrm{nm}$, nanometer; $\mathrm{NH}_{4}$, ammonium; - , no data; italic font indicates


\begin{tabular}{cccccccccccccc}
\hline $4 / 1 / 2007$ & - & - & - & - & - & 8.0 & 0.68 & 0.103 & 0.012 & 0.212 & 0.22 & 0.105 & 0.094 \\
$4 / 15 / 2007$ & 4.1 & 5.53 & - & - & - & 7.6 & 0.88 & 0.045 & 0.050 & 2.74 & 2.79 & E.0.011 & 0.315
\end{tabular} $\begin{array}{llllllllllllll}4 / 262007 & 19 & 6.06 & - & - & - & 7.6 & 1.2 & 0.175 & 0.150 & 7.48 & 7.63 & 0.084 & 0.246\end{array}$ $\begin{array}{llllllllllllll}6 / 11 / 2007 & 4.5 & 5.56 & - & - & - & 7.9 & 1.5 & 2.76 & 0.849 & 0.417 & 1.27 & 0.199 & 0.140\end{array}$ $\begin{array}{llllllllllllll}619192007 & 32 & 6.34 & - & - & - & 8.0 & 1.0 & 0.829 & 0.591 & 0.861 & 1.45 & 0.259 & 0.169\end{array}$ $\begin{array}{llllllllllllll}61992007 & 32 & 6.34 & - & - & - & 8.0 & 1.0 & 0.829 & 0.591 & 0.861 & 1.45 & 0.259 & 0.166 \\ 6 / 19 / 2007 & - & - & - & - & - & 7.8 & 1.7 & 0.657 & 0.566 & 0.814 & 1.38 & 0.117 & 0.305\end{array}$ $\begin{array}{llllllllllllll}620 / 2007 & 74 & 7.55 & - & - & - & 7.6 & 1.4 & 1.02 & 0.235 & 0.712 & 0.95 & 0.115 & 0.133\end{array}$ $\begin{array}{llllllllllllll}6120 / 2007 & 59 & 7.16 & - & - & - & 7.6 & 1.5 & 0.784 & 0.321 & 0.722 & 1.04 & 0.067 & 0.174\end{array}$ $\begin{array}{llllllllllllll}6.21 / 2007 & 42 & 6.64 & - & - & - & 7.6 & 0.99 & 0.615 & 0.326 & 0.533 & 0.86 & 0.170 & 0.193\end{array}$ \begin{tabular}{llllllllllllll}
$7 / 1 / 2007$ & 14 & 6 & - & - & - & 8.1 & 0.71 & 0.151 & 0.198 & 1.22 & 1.42 & 0.127 & 0.105 \\
\hline
\end{tabular} $\begin{array}{lllllllllllllll}77712007 & - & - & - & - & - & 7.9 & 0.82 & 0.111 & 0.043 & 1.74 & 1.78 & 0.514 & 0.214\end{array}$

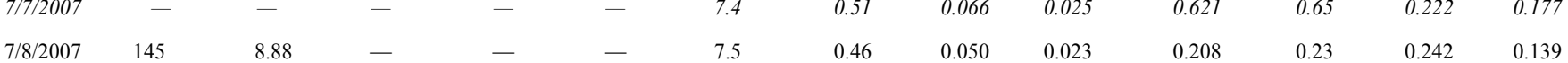
$\begin{array}{llllllllllllll}7 / 10 / 2007 & 35 & 6.41 & - & - & - & 7.0 & 0.52 & 0.101 & 0.018 & 0.070 & 0.09 & 0.398 & 0.178\end{array}$

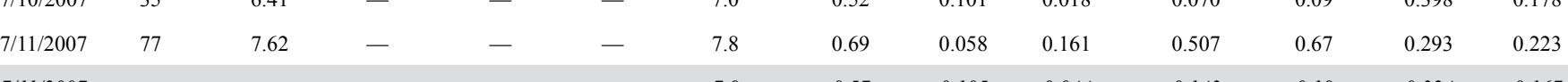
$\begin{array}{llllllllllllll}7 / 11 / 2007 & - & - & - & - & - & 7.9 & 0.57 & 0.105 & 0.044 & 0.143 & 0.19 & 0.324 & 0.167\end{array}$ $\begin{array}{llllllllllllll}7 / 1222007 & - & - & - & - & - & 7.8 & 0.51 & 0.103 & 0.034 & 0.189 & 0.22 & 0.373 & 0.200\end{array}$

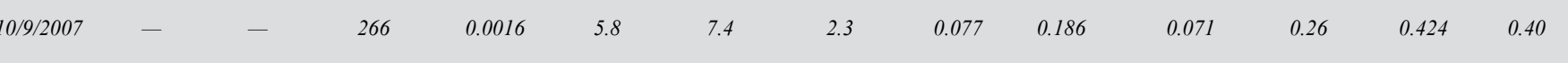

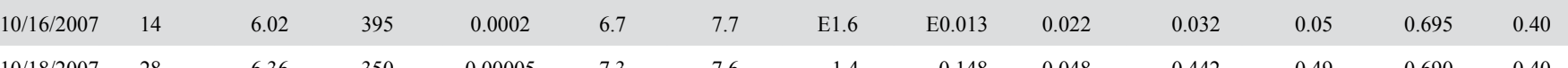
$\begin{array}{llllllllllllll}10 / 182007 & 28 & 6.36 & 350 & 0.00005 & 7.3 & 7.6 & 1.4 & 0.148 & 0.048 & 0.442 & 0.49 & 0.690 & 0.40 \\ 10 / 18 / 2007 & - & - & 335 & 0.00006 & 7.2 & 7.6 & 1.3 & 0.063 & 0.035 & 0.112 & 0.15 & 0.472 & 0.375\end{array}$ $\begin{array}{llllllll}7.5 & -0.052 & 0.016 & 1.24 & 1.26 & 0.303 & 0.184\end{array}$

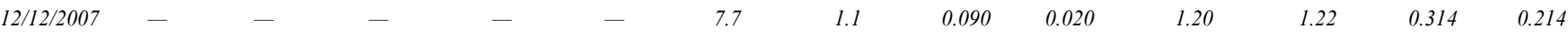

$12 / 15 / 2007$

1/10/2008

1/11/2008

1/29/2008

$2 / 2 / 2008$

$\begin{array}{llllcccccccccc}2 / 12 / 2008 & 9.4 & 5.85 & 115 & - & - & 7.7 & 1.1 & 0.031 & 0.004 & 0.221 & 0.23 & 0.073 & 0.35\end{array}$

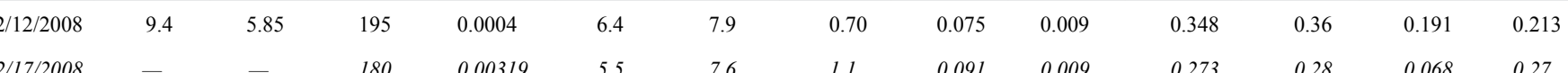

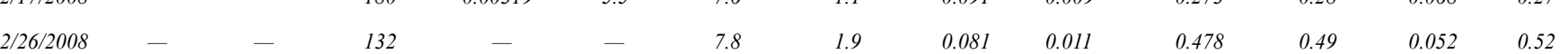

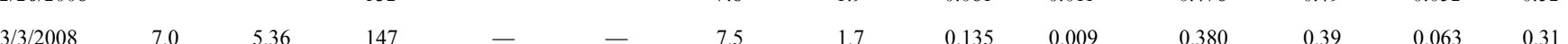

$\begin{array}{llllllllllllll}3 / 4 / 2008 & 285 & 9.71 & 52 & - & - & -7.5 & 1.7 & 0.135 & 0.009 & 0.380 & 0.39 & 0.063 & 0.31 \\ & & & & & & & & & & & \end{array}$
Table 4. Surface-water-quality data from samples collected at station 07288636, Tommie Bayou at Pace, MS. - Continued [mg/L, milligram per liter; $\mathrm{SiO}_{2}$, silica dioxide; $\mathrm{\mu g} / \mathrm{L}$, microgram per liter, UV, ultraviolet, $\mathrm{nm}$, nanometer, $\mathrm{NH}_{4}$ ammonium; - , no data; italic font indicates
composite sample; see fig, for location]

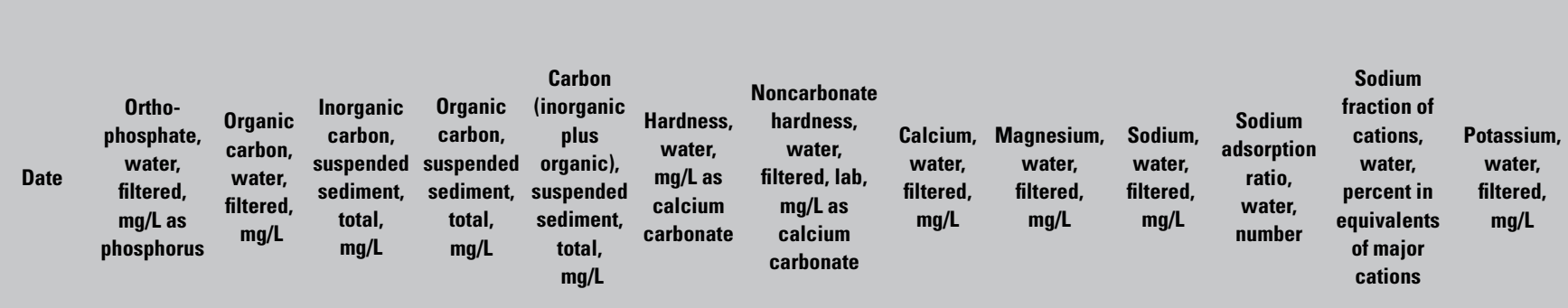

\begin{tabular}{|c|c|c|c|c|c|c|c|c|c|c|c|c|c|}
\hline $4 / 11 / 2007$ & 0.034 & 7.9 & $<0.12$ & 0.66 & 0.66 & 160 & 30 & 40.3 & 13.2 & 11.2 & 0.4 & 13 & 4.12 \\
\hline $4 / 15 / 2007$ & E0.004 & 6.3 & $<0.12$ & 3.10 & 3.17 & 78 & 35 & 21.9 & 5.74 & 6.92 & 0.3 & 16 & 2.44 \\
\hline 4/26/2007 & 0.027 & 9.7 & $<0.12$ & 1.82 & 1.82 & 150 & 100 & 40.7 & 11.6 & 11.1 & 0.4 & 14 & 4.28 \\
\hline $6 / 1112007$ & 0.065 & 8.5 & $<0.12$ & 1.03 & 1.03 & 280 & 65 & 71.0 & 25.2 & 27.5 & 0.7 & 17 & 4.63 \\
\hline $6 / 19 / 2007$ & 0.085 & 11.9 & $<0.12$ & 0.81 & 0.81 & 270 & 70 & 66.3 & 26.4 & 27.8 & 0.7 & 18 & 3.22 \\
\hline \$19/2007 & 0.038 & 8.4 & 0.27 & 16.2 & 16.5 & 250 & 59 & 61.9 & 22.7 & 24.4 & 0.7 & 17 & 2.94 \\
\hline 6/20/2007 & 0.037 & 6.0 & $<0.12$ & 1.89 & 1.90 & 160 & 39 & 40.0 & 13.4 & 12.9 & 0.4 & 15 & 2.75 \\
\hline 1201/2007 & 0.022 & 0.3 & $<0.12$ & 5.39 & 5.39 & 150 & 23 & 39.1 & 12.9 & 12.4 & 0.4 & 15 & 2.45 \\
\hline $6 / 21 / 2007$ & 0.055 & 5.0 & $<0.12$ & 32.2 & 32.2 & 170 & 35 & 43.5 & 14.8 & 14.0 & 0.5 & 15 & 2.38 \\
\hline $7 / 1 / 2007$ & 0.042 & 5.5 & $<0.12$ & 0.46 & 0.48 & 260 & 57 & 63.6 & 25.7 & 23.8 & 0.6 & 16 & 2.52 \\
\hline $7 / 7 / 2007$ & 0.168 & 4.8 & $<0.12$ & 4.25 & 4.29 & 160 & 20 & 38.9 & 14.2 & 12.6 & 0.4 & 15 & 2.87 \\
\hline $77 / 2007$ & 0.072 & 3.4 & $<0.12$ & 1.89 & 1.90 & 87 & 9 & 22.2 & 7.72 & 6.44 & 0.3 & 13 & 2.25 \\
\hline $7 / 82007$ & 0.079 & 4.2 & $<0.12$ & 0.86 & 0.88 & 74 & 5 & 19.3 & 6.16 & 4.94 & 0.3 & 12 & 2.46 \\
\hline $7 / 10 / 2007$ & 0.130 & 5.2 & $<0.12$ & 0.61 & 0.61 & 120 & 2 & 30.8 & 9.38 & 7.37 & 0.3 & 12 & 2.53 \\
\hline $7 / 11 / 2007$ & 0.096 & 4.0 & $<0.12$ & 2.24 & 2.24 & 76 & 6 & 20.5 & 6.07 & 4.80 & 0.2 & 12 & 1.95 \\
\hline $7 / 11 / 2007$ & 0.106 & 4.4 & $<0.12$ & 0.95 & 0.95 & 79 & 4 & 21.4 & 6.29 & 4.79 & 0.2 & 11 & 2.39 \\
\hline $7 / 1 / 2 / 2007$ & 0.122 & 5.2 & $<0.12$ & 1.17 & 1.18 & 99 & 4 & 26.5 & 7.83 & 5.81 & 0.3 & ${ }_{11}$ & 2.54 \\
\hline 9/5/2007 & 0.203 & 11.3 & $<0.12$ & 1.59 & 1.63 & 130 & 25 & 34.3 & 10.4 & 8.29 & 0.3 & 12 & 7.42 \\
\hline 1019/2007 & 0.138 & 23.4 & E0.04 & 5.81 & 5.85 & 110 & 17 & 28.6 & 9.10 & 6.17 & 0.3 & 10 & 10.9 \\
\hline $10 / 16 / 2007$ & 0.227 & 20.5 & E0.04 & 3.02 & 3.07 & 150 & 36 & 39.8 & 13.0 & 10.1 & 0.4 & 11 & 14.1 \\
\hline $10 / 18 / 2007$ & 0.225 & 14.8 & E0.06 & 5.83 & 5.89 & 130 & 30 & 35.4 & 11.2 & 10.1 & 0.4 & 13 & 9.40 \\
\hline $10 / 18 / 2007$ & 0.154 & 14.2 & 0.18 & 3.01 & 3.19 & 130 & 24 & 35.2 & 11.0 & 10.2 & 0.4 & 13 & 9.07 \\
\hline $11 / 27 / 2007$ & 0.099 & 7.6 & E0.11 & 1.39 & 1.50 & 120 & 53 & 32.8 & 9.49 & 10.8 & 0.4 & 16 & 4.99 \\
\hline $12 / 12 / 2007$ & 0.103 & 7.6 & $E 0.04$ & 1.42 & 1.46 & 130 & 59 & 35.5 & 10.6 & 11.2 & 0.4 & 15 & 5.70 \\
\hline $12 / 15 / 2007$ & 0.054 & 7.6 & 0.43 & 5.82 & 6.25 & 110 & 48 & 28.9 & 8.47 & 9.86 & 0.4 & 16 & 4.38 \\
\hline 1/10/2008 & 0.079 & 5.9 & $<0.04$ & 8.70 & 8.70 & 75 & 10 & 20.1 & 6.06 & 7.63 & 0.4 & 17 & 3.31 \\
\hline $1 / 11 / 2008$ & 0.062 & 6.2 & 0.47 & 8.95 & 9.42 & 52 & 9 & 13.8 & 4.22 & 5.27 & 0.3 & 17 & 2.78 \\
\hline 1/29/2008 & 0.030 & 6.8 & $E 0.08$ & 1.29 & 1.37 & 94 & 15 & 25.6 & 7.32 & 9.69 & 0.4 & 18 & 2.96 \\
\hline $2 / 1 / 12008$ & 0.028 & 3.8 & 0.31 & 4.46 & 4.77 & 48 & 4 & 13.0 & 3.70 & 4.42 & 0.3 & 16 & 1.92 \\
\hline 2/3/2008 & 0.024 & 4.0 & 0.22 & 7.49 & 7.71 & 48 & 2 & 13.1 & 3.66 & 4.58 & 0.3 & 17 & 1.99 \\
\hline 2/12/2008 & 0.062 & 6.3 & $<0.04$ & 1.85 & 1.85 & 86 & - & 23.4 & 6.74 & 6.14 & 0.3 & 13 & 2.87 \\
\hline 2/177/2008 & 0.022 & 5.7 & $<0.04$ & 5.13 & 5.13 & 73 & - & 19.7 & 5.79 & 5.88 & 0.3 & 14 & 2.19 \\
\hline 2/26/20008 & 0.017 & 4.8 & 0.32 & 15.7 & 16.0 & 52 & - & 14.1 & 4.10 & 4.64 & 0.3 & 16 & 2.04 \\
\hline $3 / 3 / 2008$ & 0.021 & 5.7 & 0.14 & 8.42 & 8.55 & 58 & - & 15.9 & 4.44 & 5.13 & 0.3 & 16 & 2.13 \\
\hline 14/2008 & 0.020 & 7.5 & 0.24 & 13.4 & 13.6 & 15 & - & 3.96 & 1.16 & 8.18 & 0.9 & 52 & 1.16 \\
\hline
\end{tabular}


Table 4. Surface-water-quality data from samples collected at station 07288636, Tommie Bayou at Pace, MS. - Continued

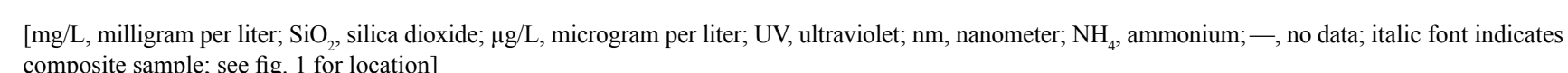

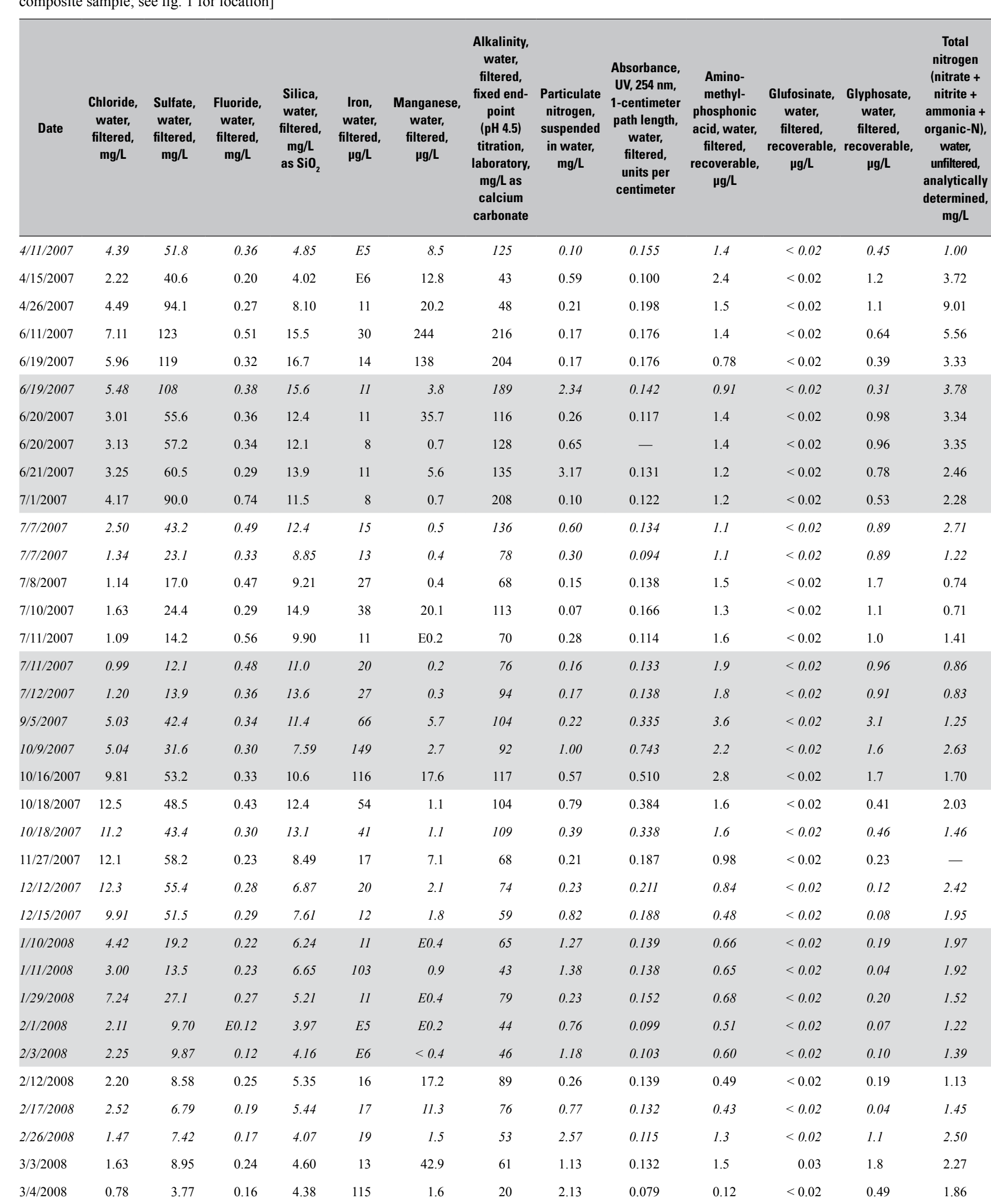

Table 4. Surface-water-quality data from samples collected at station 07288636, Tommie Bayou at Pace, MS. - Continued $\left[\mathrm{mg} / \mathrm{L}\right.$, milligram per liter; $\mathrm{SiO}_{2}$, silica dioxide; $\mu \mathrm{g} / \mathrm{L}$, microgram per liter; $\mathrm{UV}$, ultraviolet; $\mathrm{nm}$, nanometer; $\mathrm{NH}_{4}$, ammonium; - , no data; italic font indicates

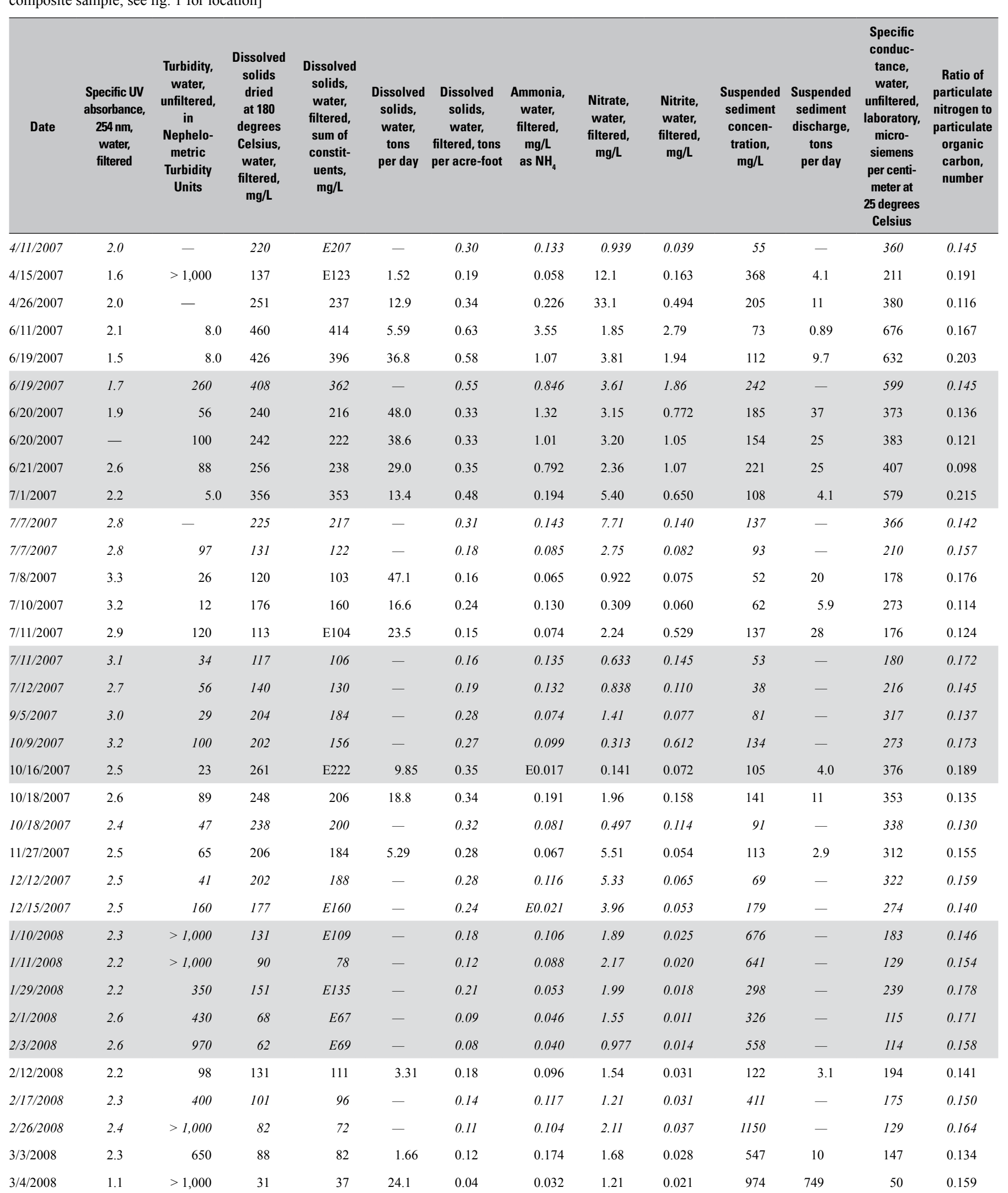


Table 4. Surface-water-quality data from samples collected at station 07288636, Tommie Bayou at Pace, MS. - Continued $\left[\mathrm{mg} / \mathrm{L}\right.$, milligram per liter, $\mathrm{SiO}_{2}$, silica dioxide; $\mu \mathrm{g} / \mathrm{L}$, microgram per liter; $\mathrm{UV}$, ultraviolet; $\mathrm{nm}$, nanometer; $\mathrm{NH}_{4}$, ammonium; - , no data; italic font indicates

\begin{tabular}{|c|c|c|c|c|c|c|c|c|c|c|c|c|c|}
\hline Date & $\begin{array}{l}\text { Discharge, } \\
\text { instanta- } \\
\text { neous. } \\
\text { cubic feet } \\
\text { per second }\end{array}$ & $\begin{array}{c}\text { Gage } \\
\text { heighth } \\
\text { feet }\end{array}$ & $\begin{array}{c}\text { Speciific } \\
\text { conductance, } \\
\text { water, } \\
\text { unfitered, } \\
\text { micro- } \\
\text { siemens } \\
\text { perc centi- } \\
\text { meter at } \\
25 \text { degrees } \\
\text { Celsius }\end{array}$ & $\begin{array}{c}\text { Hydrogen } \\
\text { ion, } \\
\text { water, } \\
\text { unifitere, } \\
\text { calculated, } \\
\text { mg/L }\end{array}$ & $\begin{array}{l}\text { pH, water, } \\
\text { untifiered, } \\
\text { field, } \\
\text { standard } \\
\text { units }\end{array}$ & $\begin{array}{c}\text { pH, water, } \\
\text { unfiftered, } \\
\text { laboratory, } \\
\text { standard } \\
\text { units }\end{array}$ & $\begin{array}{c}\text { Organic } \\
\text { nitrogen, } \\
\text { water, } \\
\text { untitered, } \\
\text { mg/L/ }\end{array}$ & $\begin{array}{l}\text { Ammonia, } \\
\text { water, } \\
\text { filtered, } \\
\text { myll Las } \\
\text { nitrogen }\end{array}$ & $\begin{array}{l}\text { Nitrite, } \\
\text { water, } \\
\text { fitererd, } \\
\text { mygl/ as } \\
\text { nitrogen }\end{array}$ & $\begin{array}{l}\text { Nitrate, } \\
\text { water, } \\
\text { filtered, } \\
\text { myll as } \\
\text { nitrogen }\end{array}$ & $\begin{array}{l}\text { Nitrate } \\
\text { plus } \\
\text { nitrite, } \\
\text { water, } \\
\text { fititered, } \\
\text { mm/l as } \\
\text { nitrogen }\end{array}$ & $\begin{array}{c}\text { Ortho- } \\
\text { phoshhate, } \\
\text { water, } \\
\text { fitered, } \\
\text { mg/L }\end{array}$ & $\begin{array}{l}\text { Phosphorus, } \\
\text { water, } \\
\text { unfitered, } \\
\text { mgl/las as } \\
\text { phosphorus }\end{array}$ \\
\hline 3/4/2008 & 197 & 9.02 & 57 & - & - & 7 & 1.4 & 0.056 & 0.008 & 0.260 & 0.27 & 0.077 & 0.40 \\
\hline 3/4/2008 & 147 & 8.49 & 68 & - & - & 7.2 & 1.2 & 0.135 & 0.017 & 0.421 & 0.44 & 0.173 & 0.42 \\
\hline 3/14/2008 & 3.8 & 7.79 & 105 & 0.00139 & 5.9 & 7.5 & 2.1 & 0.117 & 0.028 & 0.390 & 0.42 & 0.480 & 0.79 \\
\hline $3 / 14 / 2008$ & 2.7 & 7.25 & 71 & 0.00064 & 6.2 & 7.5 & E1.6 & Е0.019 & 0.006 & 0.232 & 0.24 & 0.025 & 0.38 \\
\hline 4/1/2008 & 12 & 6.17 & - & - & - & 7.4 & 1.5 & 0.050 & 0.022 & 1.95 & 1.97 & 0.101 & 0.37 \\
\hline 4/1/2008 & 67 & 7.22 & 141 & - & - & 7.4 & E1.4 & Е0.014 & 0.033 & 2.22 & 2.25 & 0.048 & 0.34 \\
\hline 4/1/2008 & - & - & 112 & - & - & 7.5 & 1.2 & 0.039 & 0.030 & 1.55 & 1.58 & 0.093 & 0.34 \\
\hline $4 / 2 / 20008$ & - & - & 142 & - & - & 7.6 & 1.2 & 0.064 & 0.018 & 0.852 & 0.87 & 0.061 & 0.31 \\
\hline 4/4/2008 & 293 & 9.69 & 88 & - & - & 7.7 & 1.1 & 0.058 & 0.011 & 0.315 & 0.33 & 0.070 & 0.36 \\
\hline 4/4/2008 & 250 & 9.38 & 85 & - & - & 7.4 & 0.86 & 0.025 & 0.010 & 0.286 & 0.30 & 0.086 & 0.28 \\
\hline $4 / 5 / 2008$ & - & - & 110 & - & - & 7.5 & 0.72 & 0.039 & 0.009 & 0.278 & 0.29 & 0.103 & 0.269 \\
\hline $4 / 26 / 2008$ & 2.4 & 5.34 & 211 & 0.00004 & 7.4 & 7.6 & 0.66 & 0.107 & 0.021 & 1.18 & 1.20 & 0.165 & 0.176 \\
\hline $4 / 26 / 2008$ & - & - & 121 & 0.00006 & 7.2 & 7.6 & 1.3 & 0.052 & 0.037 & 3.10 & 3.14 & 0.103 & 0.45 \\
\hline $5 / 2 / 2008$ & 3.1 & 5.39 & 170 & 0.00016 & 6.8 & 7.3 & 2.5 & 0.456 & 0.020 & 0.176 & 0.20 & 0.703 & 0.97 \\
\hline $5 / 3 / 2008$ & 649 & 11.41 & - & - & - & 7.4 & 0.87 & 0.047 & 0.017 & 0.345 & 0.36 & 0.110 & 0.30 \\
\hline $5 / 4 / 2008$ & 860 & 12.08 & - & - & - & 7.4 & 0.75 & 0.048 & 0.017 & 0.296 & 0.31 & 0.136 & 0.24 \\
\hline $5 / 5 / 2008$ & - & - & 94 & 0.00006 & 7.2 & 7.5 & 0.67 & 0.074 & 0.009 & 0.151 & 0.16 & 0.095 & 0.219 \\
\hline $5 / 14 / 2008$ & - & - & 132 & 0.00002 & 7.8 & 7.4 & - & 0.240 & 0.030 & 2.03 & 2.06 & 0.218 & 0.45 \\
\hline $5 / 15 / 2008$ & - & - & 151 & 0.0001 & 7.0 & 7.5 & 2.4 & 0.353 & 0.088 & 2.60 & 2.69 & 0.125 & 0.25 \\
\hline $5 / 16 / 2008$ & - & - & 171 & 0.0008 & 6.1 & 7.2 & 1.2 & 0.331 & 0.129 & 1.77 & 1.90 & 0.124 & 0.211 \\
\hline $5 / 23 / 2008$ & 0.33 & 4.69 & 180 & 0.00064 & 6.2 & 7.0 & - & $<0.020$ & 0.043 & 0.350 & 0.39 & 0.094 & 0.344 \\
\hline $5 / 23 / 2008$ & - & - & 183 & 0.0004 & 6.4 & 7.3 & 1.3 & 0.275 & 0.145 & 1.25 & 1.40 & 0.175 & 0.33 \\
\hline $619 / 2008$ & - & - & 741 & 0.00013 & 6.9 & 7.9 & 0.67 & 0.072 & 0.023 & 0.206 & 0.23 & 0.129 & 0.101 \\
\hline 7/4/2008 & 7.6 & 5.72 & 714 & - & - & 8.3 & 0.46 & 0.040 & 0.018 & 0.555 & 0.57 & 0.048 & 0.029 \\
\hline $7 / 5 / 2008$ & - & - & 609 & - & - & 8.0 & 0.83 & 0.197 & 0.321 & 1.14 & 1.46 & 0.115 & 0.084 \\
\hline $819 / 20008$ & - & - & 612 & - & - & 8.0 & 0.48 & 0.040 & 0.014 & 0.353 & 0.37 & 0.302 & 0.133 \\
\hline $8 / 19 / 2008$ & - & - & 274 & 0.00025 & 6.6 & 7.8 & - & $<0.020$ & 0.004 & 0.219 & 0.22 & 0.216 & 0.216 \\
\hline $8 / 20 / 2008$ & - & - & 188 & 0.00003 & 7.5 & 7.4 & - & $<0.020$ & 0.008 & 0.431 & 0.44 & 0.249 & 0.221 \\
\hline 9/2/20008 & - & - & 387 & 0.00064 & 6.2 & 7.7 & 0.74 & 0.033 & 0.007 & 0.174 & 0.18 & 0.280 & 0.178 \\
\hline $9 / 3 / 2008$ & - & - & 175 & 0.00032 & 6.5 & 7.4 & 0.66 & 0.035 & 0.006 & 0.152 & 0.16 & 0.324 & 0.185 \\
\hline 9/4/2008 & - & - & 185 & 0.0002 & 6.7 & 7.8 & 0.46 & 0.054 & 0.005 & 0.047 & 0.05 & 0.389 & 0.172 \\
\hline $9 / 14 / 2008$ & - & - & 347 & 0.00051 & 6.3 & 7.9 & 0.63 & 0.045 & 0.013 & 0.237 & 0.25 & 0.392 & 0.193 \\
\hline $9 / 77 / 2008$ & - & - & 489 & 0.00003 & 7.5 & 8.4 & $E 0.35$ & 0.026 & 0.007 & $E 0.023$ & $E 0.03$ & 0.200 & 0.091 \\
\hline
\end{tabular}

Table 4. Surface-water-quality data from samples collected at station 07288636, Tommie Bayou at Pace, MS. - Continued $\left[\mathrm{mg} / \mathrm{L}\right.$, milligram per liter, $\mathrm{SiO}_{2}$, silica dioxide; $\mu \mathrm{g} / \mathrm{L}$, microgram per liter, $\mathrm{UV}$, ultraviolet; $\mathrm{nm}$, nanometer, $\mathrm{NH}_{4}$ ammonium; -, no data; italic font indicates

\begin{tabular}{|c|c|c|c|c|c|c|c|c|c|c|c|c|c|}
\hline Date & $\begin{array}{c}\text { Ortho- } \\
\text { phosphate, } \\
\text { water, } \\
\text { filtered, } \\
\text { mglL as } \\
\text { phosphorus }\end{array}$ & $\begin{array}{c}\text { Organic } \\
\text { carbon, } \\
\text { water, } \\
\text { fitered, } \\
\text { mgl/ }\end{array}$ & $\begin{array}{c}\text { Inorganic } \\
\text { carbon, } \\
\text { suspended } \\
\text { sediment, } \\
\text { total, } \\
\text { mg/L }\end{array}$ & $\begin{array}{c}\text { Organic } \\
\text { carbon, } \\
\text { suspended } \\
\text { sediment, } \\
\text { total, } \\
\text { mg/L }\end{array}$ & $\begin{array}{c}\text { Carbon } \\
\text { (inorganic } \\
\text { plus } \\
\text { organicl, } \\
\text { suspended } \\
\text { sediment, } \\
\text { total. } \\
\text { mg/l }\end{array}$ & $\begin{array}{c}\text { Hardness, } \\
\text { water, } \\
\text { my/L as } \\
\text { calcium } \\
\text { carbonate }\end{array}$ & $\begin{array}{l}\text { Noncarbonate } \\
\text { hardness, } \\
\text { whater, } \\
\text { filtered, lab, } \\
\text { myll as } \\
\text { calcium } \\
\text { carbonate }\end{array}$ & $\begin{array}{c}\text { Calcium, } \\
\text { water, } \\
\text { filtered, } \\
\text { mgl/, }\end{array}$ & $\begin{array}{c}\text { Magnesium, } \\
\text { water, } \\
\text { filtered, } \\
\text { mg/L }\end{array}$ & $\begin{array}{c}\text { Sodium, } \\
\text { water, } \\
\text { fitered, } \\
\text { mgl/, }\end{array}$ & $\begin{array}{c}\text { Sodium } \\
\text { adsorproition } \\
\text { ratio, } \\
\text { water, } \\
\text { number }\end{array}$ & $\begin{array}{l}\text { Sodium } \\
\text { fraction of } \\
\text { cations, } \\
\text { water, } \\
\text { percent in } \\
\text { equivalents } \\
\text { of major } \\
\text { cations }\end{array}$ & $\begin{array}{c}\text { Potassium, } \\
\text { water, } \\
\text { filtered, } \\
\text { my/l }\end{array}$ \\
\hline 3/4/2008 & 0.025 & 9.4 & 0.18 & 8.55 & 8.73 & 17 & - & 4.67 & 1.37 & 4.18 & 0.4 & 33 & 1.26 \\
\hline 4/2008 & 0.056 & 6.3 & Е0.08 & 5.58 & 5.66 & 27 & - & 7.22 & 2.13 & 2.48 & 0.2 & 16 & 1.36 \\
\hline 3/14/2008 & 0.157 & 4.9 & 0.41 & 15.0 & 15.4 & 31 & - & 9.68 & 1.66 & 7.19 & 0.6 & 32 & 2.09 \\
\hline 3/14/2008 & 0.008 & 3.1 & 0.56 & 11.2 & 11.8 & 25 & 1 & 6.61 & 2.00 & 3.14 & 0.3 & 21 & 1.20 \\
\hline 4/1/2008 & 0.033 & 7.0 & E0.05 & 4.62 & 4.67 & 68 & 16 & 18.3 & 5.41 & 5.87 & 0.3 & 15 & 2.70 \\
\hline 4/1/2008 & 0.016 & 5.9 & 0.49 & 7.57 & 8.05 & 51 & 22 & 13.8 & 3.96 & 5.38 & 0.3 & 18 & 2.30 \\
\hline $1 / 1 / 2008$ & 0.030 & 5.7 & 0.23 & 6.11 & 6.34 & 42 & 15 & 11.3 & 3.23 & 4.26 & 0.3 & 17 & 2.13 \\
\hline 4/2/2008 & 0.020 & 5.7 & $<0.04$ & 6.05 & 6.05 & 50 & 15 & 13.6 & 3.96 & 5.28 & 0.3 & 18 & 2.14 \\
\hline 4/4/2008 & 0.023 & 4.1 & $<0.04$ & 7.40 & 7.40 & 31 & 3 & 8.41 & 2.47 & 3.77 & 0.3 & 20 & 1.69 \\
\hline 4/4/2008 & 0.028 & 4.3 & $<0.04$ & 4.13 & 4.13 & 31 & 0 & 8.48 & 2.44 & 3.23 & 0.3 & 17 & 1.69 \\
\hline $4 / 5 / 2008$ & 0.034 & 5.0 & $<0.04$ & 2.91 & 2.91 & 40 & 0 & 10.8 & 3.20 & 4.02 & 0.3 & 17 & 1.75 \\
\hline 4/2612008 & 0.054 & 7.2 & Е0.10 & 1.58 & 1.68 & 84 & 19 & 22.3 & 6.88 & 6.75 & 0.3 & 14 & 3.60 \\
\hline $4 / 2662008$ & 0.034 & 5.6 & 0.15 & 12.1 & 12.3 & 45 & 19 & 12.1 & 3.50 & 4.07 & 0.3 & 16 & 2.16 \\
\hline $5 / 2 / 2008$ & 0.229 & 6.1 & 0.49 & 25.5 & 26.0 & 38 & - & 12.1 & 1.98 & 13.5 & 0.9 & 41 & 3.30 \\
\hline $5 / 3 / 2008$ & 0.036 & 2.9 & Е0.05 & 5.98 & 6.03 & 11 & - & 2.85 & 0.865 & 1.23 & 0.2 & 18 & 1.09 \\
\hline $5 / 4 / 2008$ & 0.044 & 3.8 & E0.04 & 3.86 & 3.90 & 15 & - & 4.07 & 1.21 & 1.44 & 0.2 & 16 & 1.33 \\
\hline $5 / 5 / 2008$ & 0.031 & 5.9 & $<0.04$ & 2.13 & 2.13 & 34 & - & 9.31 & 2.73 & 3.08 & 0.2 & 15 & 1.94 \\
\hline $5 / 14 / 2000$ & 0.071 & 6.3 & $E 0.09$ & 6.23 & 6.32 & 46 & 12 & 12.9 & 3.43 & 4.74 & 0.3 & 17 & 2.70 \\
\hline $5 / 15 / 2008$ & 0.041 & 7.5 & $<0.04$ & 5.03 & 5.03 & 53 & 20 & 14.6 & 4.10 & 5.10 & 0.3 & 16 & 2.61 \\
\hline $5 / 16 / 2008$ & 0.040 & - & $<0.04$ & 1.92 & 1.92 & 63 & 22 & 17.3 & 4.84 & 5.11 & 0.3 & 14 & 2.71 \\
\hline $5 / 23 / 2008$ & 0.031 & 7.3 & $<0.04$ & 6.53 & 6.53 & 68 & 21 & 18.7 & 5.28 & 5.22 & 0.3 & 14 & 3.04 \\
\hline $5 / 23 / 2008$ & 0.057 & 7.3 & 0.14 & 5.36 & 5.49 & 70 & 18 & 19.3 & 5.28 & 5.42 & 0.3 & 14 & 2.89 \\
\hline 6/19/2008 & 0.042 & 7 & $<0.04$ & 1.12 & 1.12 & 320 & 110 & 83.8 & 27.5 & 31.9 & 0.8 & 17 & 4.31 \\
\hline 7/4/2008 & 0.016 & 5.3 & 1.36 & 3.04 & 4.40 & 330 & 88 & 80.7 & 31.6 & 30.3 & 0.7 & 16 & 3.85 \\
\hline $7 / 5 / 2008$ & 0.037 & 6.7 & 0.14 & 1.24 & 1.38 & 270 & 73 & 70.5 & 23.9 & 24.5 & 0.6 & 16 & 4.17 \\
\hline $89 / 2008$ & 0.098 & 5.3 & $<0.04$ & 1.07 & 1.07 & 270 & 48 & 68.9 & 24.9 & 22.3 & 0.6 & 15 & 4.20 \\
\hline $8 / 19 / 20008$ & 0.070 & 6.0 & $<0.04$ & 3.31 & 3.31 & 120 & 19 & 29.6 & 10.8 & 9.97 & 0.4 & 15 & 3.73 \\
\hline $8 / 20 / 2008$ & 0.081 & 6.2 & $<0.04$ & 1.74 & 1.74 & 75 & - & 19.6 & 6.19 & 6.02 & 0.3 & 14 & 4.06 \\
\hline $1 / 2 / 20008$ & 0.091 & 7.5 & $E 0.03$ & 2.04 & 2.07 & 170 & 16 & 43.0 & 15.0 & 13.5 & 0.5 & 14 & 4.66 \\
\hline 9/3/2008 & 0.106 & 7.4 & $<0.04$ & 2.42 & 2.42 & 72 & 0 & 19.3 & 5.80 & 4.74 & 0.2 & 12 & 4.91 \\
\hline $1 / 4 / 2008$ & 0.127 & 6.1 & $<0.04$ & 0.88 & 0.88 & 77 & - & 20.8 & 6.21 & 4.24 & 0.2 & 10 & 4.98 \\
\hline $1 / 14 / 2008$ & 0.128 & 6.9 & $<0.04$ & 1.61 & 1.61 & 150 & 11 & 39.2 & 13.1 & 11.4 & 0.4 & 14 & 5.71 \\
\hline $177 / 2008$ & 0.065 & 5.7 & $<0.04$ & 0.51 & 0.51 & 230 & 34 & 59.0 & 19.5 & 18.4 & 0.5 & 15 & 5.90 \\
\hline
\end{tabular}


Table 4. Surface-water-quality data from samples collected at station 07288636, Tommie Bayou at Pace, MS. - Continued [mg/L, milligram per liter; $\mathrm{SiO}_{2}$, silica dioxide; $\mu \mathrm{g} \mathrm{L}$, microgram per liter; $\mathrm{UV}$, ultraviolet; nm, nanometer; $\mathrm{NH}_{\downarrow}$, ammonium; -, no data; ; italic font indicates
composite sample; see fig. 1 for location]

\begin{tabular}{|c|c|c|c|c|c|c|c|c|c|c|c|c|c|}
\hline Date & $\begin{array}{c}\text { Chloride, } \\
\text { water, } \\
\text { fitered, } \\
\text { myll }\end{array}$ & $\begin{array}{c}\text { Sulfate, } \\
\text { water, } \\
\text { fitiered, } \\
\text { mgl/ }\end{array}$ & $\begin{array}{c}\text { Fluoride, } \\
\text { water, } \\
\text { filtered, } \\
\text { mg/L }\end{array}$ & 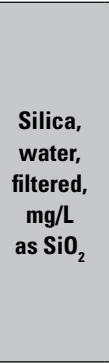 & $\begin{array}{c}\text { Iron, } \\
\text { water, } \\
\text { fititered, } \\
\text { pg/L }\end{array}$ & $\begin{array}{c}\text { Manganese, } \\
\text { water, } \\
\text { filtered, } \\
\text { pg/L/L }\end{array}$ & 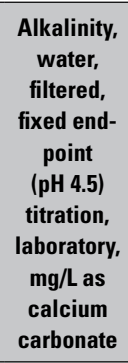 & $\begin{array}{c}\text { Particulate } \\
\text { introgen, } \\
\text { suspended } \\
\text { in water, } \\
\text { mg/L }\end{array}$ & 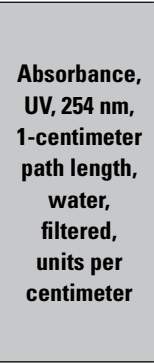 & $\begin{array}{c}\text { Amino- } \\
\text { methyl- } \\
\text { phosphhonic } \\
\text { acid, water, } \\
\text { iflered, } \\
\text { recoverable, } \\
\text { ygl/ }\end{array}$ & $\begin{array}{l}\text { Glutosinate, } \\
\text { water, } \\
\text { fitited, } \\
\text { recoverable, } \\
\text { yg/L }\end{array}$ & $\begin{array}{l}\text { Glyphosate, } \\
\text { water., } \\
\text { fitered, } \\
\text { recoverable, } \\
\text { yg/L }\end{array}$ & $\begin{array}{c}\text { Total } \\
\text { nitrogen } \\
\text { (nitrate + } \\
\text { nitrite + } \\
\text { ammonia + } \\
\text { organic-N), } \\
\text { water, } \\
\text { unfitered, } \\
\text { analytically } \\
\text { determined, } \\
\text { mg/ll }\end{array}$ \\
\hline 3/4/2008 & 0.65 & 3.48 & 0.15 & 3.14 & 68 & 3.2 & 21 & 1.48 & 0.079 & 0.69 & $<0.02$ & 0.53 & 1.73 \\
\hline 3/4/2008 & 0.85 & 5.49 & 0.18 & 3.00 & 38 & 6.9 & 29 & 0.99 & 0.091 & 0.99 & $<0.02$ & 1.1 & 1.75 \\
\hline 14/2008 & 4.22 & 4.55 & E0.10 & 3.10 & 60 & 0.6 & 41 & 1.71 & 0.139 & 1.4 & $<0.02$ & 1.2 & 2.61 \\
\hline 3/14/2000 & 1.09 & 8.30 & 0.15 & 2.40 & 26 & E0.2 & 24 & 1.57 & 0.069 & 0.77 & $<0.02$ & 0.32 & 1.81 \\
\hline 1/2008 & 3.31 & 20.0 & 0.25 & 5.53 & 12 & $<0.4$ & 52 & 0.70 & 0.168 & 2.6 & $<0.02$ & 3.0 & 3.47 \\
\hline 4/1/2008 & 3.05 & 23.5 & 0.20 & 5.35 & 16 & E0.3 & 29 & 1.22 & 0.138 & 2.0 & $<0.02$ & 1.1 & 3.71 \\
\hline 4/1/2008 & 1.88 & 18.3 & 0.16 & 5.24 & 15 & 1.6 & 26 & 0.94 & 0.138 & 1.8 & $<0.02$ & 1.2 & 2.87 \\
\hline 4/2/2008 & 2.66 & 24.7 & 0.23 & 5.94 & 14 & $E 0.4$ & 35 & 0.99 & 0.133 & 1.9 & $<0.02$ & 0.96 & 2.13 \\
\hline 4/4/2008 & 1.49 & 11.2 & 0.37 & 4.57 & 13 & E0.3 & 28 & 1.06 & 0.099 & 1.3 & $<0.02$ & 0.41 & 1.51 \\
\hline 4/4/2008 & 1.16 & 8.68 & 0.15 & 4.60 & 18 & Е0.4 & 30 & 0.56 & 0.113 & 1.5 & 0.02 & 0.75 & 1.18 \\
\hline $4 / 5 / 2008$ & 1.31 & 11.5 & 0.32 & 5.48 & 16 & $E 0.3$ & 40 & 0.52 & 0.124 & 1.7 & $<0.02$ & 0.99 & 1.05 \\
\hline 4/26/2008 & 2.83 & 26.9 & 0.20 & 3.90 & 12 & 3.4 & 65 & 0.32 & 0.145 & 1.3 & $<0.02$ & 0.85 & 1.97 \\
\hline 4/26/2008 & 1.68 & 16.6 & 0.17 & 6.52 & 9 & 0.5 & 26 & 1.86 & 0.140 & 1.1 & $<0.02$ & 0.43 & 4.50 \\
\hline $5 / 2 / 2008$ & 8.67 & 7.93 & 0.26 & 4.71 & 49 & 24.1 & 53 & 2.91 & 0.173 & 1.8 & $<0.02$ & 3.5 & 3.10 \\
\hline $5 / 3 / 2008$ & 0.52 & 3.45 & 0.22 & 4.21 & 117 & 1.0 & 11 & 0.94 & 0.080 & 0.82 & $<0.02$ & 0.41 & 1.28 \\
\hline $5 / 4 / 2008$ & 0.61 & 3.98 & 0.19 & 5.01 & 73 & 0.8 & 17 & 0.63 & 0.107 & 1.0 & $<0.02$ & 0.48 & 1.11 \\
\hline $5 / 5 / 2008$ & 0.94 & 8.05 & 0.24 & 6.86 & 30 & 0.9 & 35 & 0.32 & 0.149 & 0.96 & $<0.02$ & 0.20 & 0.91 \\
\hline $5 / 14 / 2008$ & 1.67 & 15.0 & 0.20 & 6.37 & 33 & 7.0 & 35 & 0.91 & 0.174 & 2.4 & 0.02 & 6.2 & 1.54 \\
\hline $5 / 15 / 2008$ & 1.53 & 22.0 & 0.25 & 7.92 & 16 & 1.3 & 33 & 0.97 & 0.177 & 1.9 & 0.02 & 1.5 & 5.41 \\
\hline $5 / 16 / 2008$ & 1.72 & 28.7 & 0.19 & 8.80 & 12 & 1.7 & 41 & 0.36 & - & - & - & - & 3.47 \\
\hline $5 / 23 / 2000$ & 1.88 & 31.9 & 0.33 & 1.96 & 16 & E0.3 & 48 & 1.08 & 0.170 & 1.3 & $<0.02$ & 0.23 & 1.63 \\
\hline $5 / 23 / 2008$ & 2.49 & 25.9 & 0.29 & 8.10 & 11 & 1.1 & 52 & 0.94 & 0.194 & 1.7 & $<0.02$ & 1.5 & 2.95 \\
\hline 6/9/2008 & 11.4 & 172 & 0.37 & 11.6 & 38 & 37.2 & 214 & 0.11 & 0.168 & 1.9 & $<0.02$ & 0.77 & 0.97 \\
\hline 7/4/2008 & 9.14 & 148 & 0.36 & 8.35 & 21 & 1.1 & 244 & 0.15 & 0.119 & 3.5 & $<0.02$ & 0.92 & 1.08 \\
\hline 7/5/2008 & 7.77 & 117 & 0.43 & 13.6 & $E \sigma$ & 0.5 & 202 & 0.16 & 0.156 & 4.4 & $<0.02$ & 4.0 & 2.49 \\
\hline 8/9/2008 & 6.52 & 87.7 & 0.30 & 17.7 & 16 & 1.4 & 226 & 0.13 & 0.140 & 1.9 & $<0.02$ & 0.88 & 0.88 \\
\hline $8 / 19 / 2008$ & 2.66 & 23.4 & 0.20 & 10.0 & 24 & 0.8 & 100 & 0.36 & 0.171 & 3.0 & $<0.02$ & 0.85 & 1.01 \\
\hline 8/20/2008 & 2.28 & 17.8 & 0.17 & 8.17 & 35 & 0.8 & 75 & 0.33 & 0.187 & 3.2 & $<0.02$ & 1.3 & 1.22 \\
\hline 9/2/2008 & 4.52 & 40.5 & 0.39 & 12.9 & 30 & 0.7 & 153 & 0.32 & 0.226 & 1.9 & $<0.02$ & 0.54 & 0.95 \\
\hline 9/3/2008 & 2.33 & 13.3 & 0.18 & 9.39 & 46 & 1.5 & 71 & 0.31 & 0.221 & 1.6 & $<0.02$ & 0.60 & 0.85 \\
\hline 9/4/2008 & 2.66 & 12.2 & 0.20 & 10.8 & 38 & $E 0.3$ & 79 & 0.12 & 0.179 & 1.5 & $<0.02$ & 0.53 & 0.57 \\
\hline 9/14/2008 & 6.45 & 33.4 & 0.25 & 13.5 & 25 & 1.0 & 141 & 0.23 & 0.206 & 1.5 & $<0.02$ & 0.37 & 0.93 \\
\hline 7/2008 & 0.5 & 57.3 & 0.29 & 14.8 & 12 & 0.3 & 193 & 0.08 & 0.132 & 1.4 & $<0.02$ & 0.19 & 0.41 \\
\hline
\end{tabular}

Table 4. Surface-water-quality data from samples collected at station 07288636, Tommie Bayou at Pace, MS.-Continued $\left[\mathrm{mg} / \mathrm{L}\right.$, milligram per liter; $\mathrm{SiO}_{2}$, silica dioxide; $\mu \mathrm{g} / \mathrm{L}$, microgram per liter; $\mathrm{UV}$, ultraviolet; $\mathrm{nm}$, nanometer; $\mathrm{NH}_{4}$, ammonium; --, no data; italic font indicates

\begin{tabular}{|c|c|c|c|c|c|c|c|c|c|c|c|c|c|}
\hline Date & $\begin{array}{l}\text { Specific UV } \\
\text { absoronance, } \\
\text { 254nu, } \\
\text { water, } \\
\text { fithered }\end{array}$ & $\begin{array}{l}\text { Turbiditity, } \\
\text { water, } \\
\text { unfilitered, } \\
\text { Nen } \\
\text { Nephelo- } \\
\text { metric } \\
\text { Turbiditity } \\
\text { Units }\end{array}$ & $\begin{array}{l}\text { Dissolved } \\
\text { solids } \\
\text { dried } \\
\text { at 1880 } \\
\text { degres } \\
\text { celsius, } \\
\text { water, } \\
\text { filtered, } \\
\text { mgl/L }\end{array}$ & 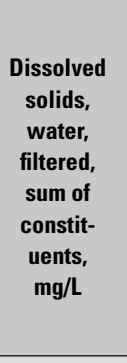 & $\begin{array}{c}\text { Dissolved } \\
\text { solids, } \\
\text { water, } \\
\text { tons } \\
\text { per day }\end{array}$ & $\begin{array}{c}\text { Dissolved } \\
\text { solidst, } \\
\text { water, } \\
\text { filtered, tons } \\
\text { per acre-foot }\end{array}$ & $\begin{array}{l}\text { Ammonia, } \\
\text { water, } \\
\text { filtered, } \\
\text { mgy/L } \\
\text { as } \mathrm{NH}_{4}\end{array}$ & $\begin{array}{c}\text { Nitrate, } \\
\text { water, } \\
\text { fitiered, } \\
\text { mg/L }\end{array}$ & $\begin{array}{c}\text { Nitrite, } \\
\text { water, } \\
\text { fititered, } \\
\text { mg/L }\end{array}$ & $\begin{array}{c}\text { Suspended } \\
\text { sediment } \\
\text { concen- } \\
\text { tration, } \\
\text { my/L }\end{array}$ & $\begin{array}{c}\text { Suspended } \\
\text { sediement } \\
\text { discharge, } \\
\text { tons } \\
\text { per day }\end{array}$ & $\begin{array}{c}\text { Specific } \\
\text { conduc- } \\
\text { tance, } \\
\text { water, } \\
\text { unfiltered, } \\
\text { laboratory, } \\
\text { microsie- } \\
\text { mens per } \\
\text { centimeter at } \\
\text { 25 Cegrees } \\
\text { Celsius }\end{array}$ & $\begin{array}{c}\text { Ratio of } \\
\text { partituculate } \\
\text { nititgenen to } \\
\text { partitulate } \\
\text { organic } \\
\text { carbon, } \\
\text { number }\end{array}$ \\
\hline 3/4/2008 & 0.8 & $>1,000$ & 40 & 33 & 21.2 & 0.05 & 0.072 & 1.15 & 0.026 & 665 & 354 & 51 & 0.173 \\
\hline 4/2008 & 1.5 & 820 & 51 & 42 & 20.4 & 0.07 & 0.173 & 1.86 & 0.057 & 492 & 195 & 72 & 0.177 \\
\hline 3/14/2008 & 2.8 & 780 & 61 & E60 & 0.62 & 0.08 & 0.151 & 1.72 & 0.093 & 723 & 7.4 & 111 & 0.114 \\
\hline 3/14/2008 & 2.3 & $>1,000$ & 45 & E40 & 0.33 & 0.06 & Е0.024 & 1.03 & 0.019 & 1080 & 7.9 & 73 & 0.140 \\
\hline 4/1/2008 & 2.4 & $>1,000$ & 119 & 101 & 3.86 & 0.16 & 0.064 & 8.63 & 0.071 & 702 & 23 & 176 & 0.152 \\
\hline /1/2008 & 2.3 & 950 & 97 & E85 & 17.5 & 0.13 & Е0.018 & 9.83 & 0.107 & 616 & 111 & 146 & 0.161 \\
\hline (1//2008 & 2.4 & 810 & 81 & 69 & - & 0.11 & 0.050 & 6.87 & 0.097 & - & - & 116 & 0.154 \\
\hline 4/2/20008 & 2.4 & 710 & 96 & $E 84$ & - & 0.13 & 0.082 & 3.77 & 0.058 & 465 & - & 144 & 0.163 \\
\hline 4/4/2008 & 2.4 & 840 & 64 & E52 & 50.9 & 0.09 & 0.075 & 1.39 & 0.036 & 523 & 414 & 91 & 0.144 \\
\hline 4/4/2008 & 2.6 & 460 & 59 & E50 & 39.9 & 0.08 & 0.033 & 1.27 & 0.032 & 328 & 221 & 86 & 0.134 \\
\hline 4/5/2008 & 2.5 & 240 & 70 & E64 & - & 0.10 & 0.050 & 1.23 & 0.029 & 201 & - & 111 & 0.178 \\
\hline 4/26/2008 & 2.0 & 86 & 139 & 118 & 0.90 & 0.19 & 0.138 & 5.22 & 0.069 & 118 & 0.76 & 206 & 0.202 \\
\hline 4/26/2008 & 2.5 & $>1,000$ & 90 & 77 & - & 0.12 & 0.066 & 13.7 & 0.123 & 661 & - & 120 & 0.153 \\
\hline 5/2/2008 & 2.8 & 840 & 103 & 87 & 0.86 & 0.14 & 0.587 & 0.777 & 0.067 & 826 & 6.9 & 156 & 0.114 \\
\hline 5//2008 & 2.8 & 750 & 25 & 23 & 44.4 & 0.03 & 0.061 & 1.53 & 0.055 & 435 & 762 & 36 & 0.158 \\
\hline 5/4/2008 & 2.8 & 440 & 47 & 30 & 110 & 0.06 & 0.062 & 1.31 & 0.056 & 289 & 671 & 47 & 0.164 \\
\hline $5 / 5 / 2008$ & 2.5 & 200 & 73 & 55 & - & 0.10 & 0.095 & 0.669 & 0.029 & 157 & - & 91 & 0.149 \\
\hline $5 / 14 / 2008$ & 2.7 & 370 & 84 & 77 & - & 0.11 & 0.308 & 8.99 & 0.097 & 235 & - & 100 & 0.146 \\
\hline 5/15/2008 & 2.3 & 500 & 110 & 90 & - & 0.15 & 0.454 & 11.5 & 0.289 & 346 & - & 146 & 0.193 \\
\hline $5 / 16 / 2008$ & - & 160 & 123 & 103 & - & 0.17 & 0.426 & 7.85 & 0.423 & 165 & - & 172 & 0.186 \\
\hline $5 / 23 / 2008$ & 2.3 & 280 & 103 & E99 & 0.09 & 0.14 & $<0.026$ & 1.55 & 0.140 & 279 & 0.25 & 180 & 0.166 \\
\hline $5 / 23 / 2008$ & 2.7 & - & 118 & 108 & - & 0.16 & 0.354 & 5.54 & 0.475 & 425 & - & 183 & 0.176 \\
\hline 6/9/2008 & 2.4 & 19 & 514 & 472 & - & 0.70 & 0.093 & 0.912 & 0.076 & 96 & - & 749 & 0.100 \\
\hline 7/4/2008 & 2.3 & 3.0 & 491 & 461 & 10.1 & 0.67 & 0.052 & 2.46 & 0.060 & 106 & 2.2 & 730 & 0.048 \\
\hline 7/5/20088 & 2.3 & 10 & 426 & $E 389$ & - & 0.58 & 0.253 & 5.04 & 1.05 & 76 & - & 622 & 0.130 \\
\hline 8/9/2008 & 2.6 & 10 & 384 & 370 & - & 0.52 & 0.051 & 1.56 & 0.047 & 57 & - & 578 & 0.119 \\
\hline $8 / 19 / 2008$ & 2.9 & 79 & 153 & 151 & - & 0.21 & $<0.026$ & 0.968 & 0.014 & 102 & - & 280 & 0.108 \\
\hline $8 / 20 / 2008$ & 3.0 & 67 & 121 & 111 & - & 0.16 & $<0.026$ & 1.91 & 0.027 & 88 & - & 193 & 0.188 \\
\hline 9/2/20008 & 3.0 & 30 & 247 & 227 & - & 0.34 & 0.043 & 0.772 & 0.022 & 232 & - & 387 & 0.157 \\
\hline 9/3/2008 & 3.0 & 33 & 109 & 104 & - & 0.15 & 0.045 & 0.673 & 0.020 & 157 & - & 176 & 0.127 \\
\hline 1/4/2008 & 2.9 & 7.0 & 112 & E110 & - & 0.15 & 0.069 & 0.208 & 0.016 & 105 & - & 188 & 0.138 \\
\hline 9/14/2008 & 3.0 & 20 & 228 & 209 & - & 0.31 & 0.058 & 1.05 & 0.043 & 184 & - & 359 & 0.143 \\
\hline /177/2008 & 2.3 & 4.4 & 317 & E302 & - & 0.43 & 0.033 & $E 0.101$ & 0.022 & 153 & - & 496 & 0.164 \\
\hline
\end{tabular}




\section{Data Associated with the Mississippi Embayment Agricultural Chemical Transport Study, 2006-2008}

Table 5. Surface-water-quality data from samples collected at station 333150090530400, an unnamed tributary to Clear Creek near Napanee, MS.

[mg L, milligram per liter; $\mathrm{SiO}_{2}$, silica dioxide; $\mu \mathrm{g} / \mathrm{L}$, microgram per liter; $\mathrm{UV}$, ultraviolet; $\mathrm{nm}$, nanometer; $\mathrm{NH}_{4}$, ammonium; -, no data; italic font indicates composite sample; see fig. 1 for location]



\begin{tabular}{|c|c|c|c|c|c|c|c|c|c|c|c|c|c|}
\hline $4 / 142007$ & 0.07 & 3.95 & - & - & - & 7.4 & 3.9 & 0.078 & 0.037 & 5.29 & 5.33 & 0.083 & 0.74 \\
\hline 4/144/2007 & - & - & - & - & - & 7.7 & 2.4 & 0.064 & 0.131 & 8.76 & 8.89 & 0.038 & 0.54 \\
\hline $4 / 262007$ & 0.19 & 4.12 & - & - & - & 7.5 & E1.5 & E0.011 & 0.039 & 3.90 & 3.94 & E0.015 & 0.24 \\
\hline $5 / 42007$ & - & - & - & - & - & 6.5 & 3.3 & 0.060 & 0.052 & 8.06 & 8.11 & 0.054 & 0.71 \\
\hline $5 / 22 / 2007$ & - & - & 793 & - & - & 8.3 & 0.88 & 0.027 & 1.72 & 1.68 & 3.40 & 0.115 & 0.080 \\
\hline $69 / 2007$ & - & - & - & - & - & 8.2 & ${ }_{11}$ & 1.69 & 0.050 & 0.998 & 1.05 & 0.076 & 0.069 \\
\hline $6 / 19 / 2007$ & 3.4 & 5.14 & - & - & - & 7.6 & 11 & 2.36 & 0.565 & 2.95 & 3.52 & 0.151 & 0.304 \\
\hline $6 / 19 / 2007$ & 17 & 7.03 & - & - & - & 7.4 & 4 & 0.570 & 0.113 & 1.29 & 1.41 & 0.165 & 0.51 \\
\hline $6 / 19 / 2007$ & 18 & 7.20 & - & - & - & 7.5 & 5.9 & 0.911 & 0.126 & 1.06 & 1.18 & 0.126 & 0.322 \\
\hline 6/19/2007 & 18 & 7.07 & - & - & - & 7.4 & 7.8 & 1.26 & 0.102 & 0.713 & 0.81 & 0.131 & 0.208 \\
\hline $6 / 19 / 2007$ & 21 & 7.50 & - & - & - & 7.3 & 8.1 & 1.35 & 0.091 & 0.918 & 1.01 & 0.132 & 0.392 \\
\hline 6/19/2007 & 20 & 7.53 & - & - & - & 7.3 & 7.3 & 1.33 & 0.085 & 0.678 & 0.76 & 0.107 & 0.274 \\
\hline $6 / 19 / 2007$ & 19 & 7.35 & - & - & - & 7.5 & 8.4 & 1.64 & 0.084 & 0.538 & 0.62 & 0.087 & 0.163 \\
\hline 6/20/2007 & 18 & 7.09 & - & - & - & 7.6 & 8.4 & 1.79 & 0.083 & 0.432 & 0.52 & 0.086 & 0.128 \\
\hline 6/20/2007 & 11 & 6.31 & 387 & 0.00006 & 7.2 & 7.6 & 4.3 & 2.76 & 2.45 & 1.56 & 4.01 & 0.067 & 0.119 \\
\hline $6 / 21 / 2007$ & 7.9 & 5.84 & 484 & 0.000003 & 7.5 & 7.6 & 2.8 & 4.44 & 4.61 & 1.19 & 5.80 & 0.046 & 0.064 \\
\hline $6 / 21 / 2007$ & - & - & 510 & 0.00003 & 7.6 & 7.8 & 1.1 & 4.81 & 3.03 & 1.18 & 4.20 & 0.030 & 0.043 \\
\hline $6 / 26 / 2007$ & - & - & 273 & - & - & 8.0 & El.1 & $E 0.011$ & 0.022 & 2.40 & 2.42 & 0.120 & 0.25 \\
\hline $6 / 27 / 2007$ & - & - & 375 & - & - & 8.2 & $E 0.75$ & $E 0.011$ & 0.023 & 1.31 & 1.33 & 0.109 & 0.112 \\
\hline 7/7/2007 & 5.5 & 5.49 & - & - & - & 7.4 & 3.5 & 0.026 & 0.034 & 1.06 & 1.09 & 0.258 & 0.57 \\
\hline $7 / 72007$ & 20 & 7.37 & - & - & - & 7.7 & 0.71 & 0.036 & 0.012 & 0.646 & 0.66 & 0.122 & 0.288 \\
\hline $7 / 72007$ & 22 & 7.69 & - & - & - & 7.9 & - & $<0.020$ & 0.017 & 0.316 & 0.33 & 0.092 & 0.187 \\
\hline $7 / 7 / 2007$ & - & - & - & - & - & 8.2 & 0.54 & 0.034 & 0.007 & 0.226 & 0.23 & 0.095 & 0.118 \\
\hline $79 / 2007$ & 6.2 & 5.59 & - & - & - & 8.0 & 0.59 & 0.047 & 0.082 & 0.176 & 0.26 & 0.068 & 0.108 \\
\hline $7 / 10 / 2007$ & 6.2 & 5.60 & - & - & - & 8.0 & 0.54 & 0.038 & 0.041 & 0.075 & 0.12 & 0.073 & 0.090 \\
\hline 8/2/2007 & 10 & 6.15 & 634 & - & - & 8.2 & E0.58 & E0.013 & 0.149 & 0.185 & 0.33 & 0.147 & 0.118 \\
\hline $8 / 22007$ & 11 & 6.27 & 643 & - & - & 8.3 & E0.48 & E0.011 & 0.090 & 0.139 & 0.23 & 0.155 & 0.096 \\
\hline $82 / 2007$ & - & - & 727 & - & - & 8.3 & 0.35 & 0.077 & 0.030 & 0.047 & 0.08 & 0.117 & 0.069 \\
\hline $8 / 11 / 2007$ & 2.7 & 5.02 & 530 & 0.00010 & 7.0 & 7.6 & 1.2 & 0.055 & 0.016 & 0.054 & 0.07 & 0.622 & 0.34 \\
\hline 3/42008 & 32 & 7.40 & 49 & - & - & 7 & E1.0 & E0.020 & 0.006 & 0.15 & 0.15 & 0.206 & 0.36 \\
\hline 3/42008 & 30 & 7.22 & 49 & 0.00010 & 7.0 & 7.7 & - & $<0.020$ & $<0.002$ & - & 0.14 & 0.072 & 0.12 \\
\hline 3/5/2008 & 15 & 6.18 & 68 & 0.00006 & 7.2 & 7.3 & - & $<0.020$ & 0.008 & 0.146 & 0.15 & 0.179 & 0.11 \\
\hline $3 / 5 / 2008$ & - & - & 82 & 0.000006 & 7.2 & 7.3 & - & $<0.020$ & 0.025 & 0.174 & 0.20 & 0.119 & 0.13 \\
\hline $4 / 12008$ & 16 & 6.32 & 119 & - & - & 7.5 & 3.3 & 0.024 & 0.028 & 2.79 & 2.82 & 0.085 & 0.79 \\
\hline /2008 & 33 & 7.40 & 64 & - & - & & 1.4 & 0.013 & & & & 1.165 & 0.47 \\
\hline
\end{tabular}

Table 5. Surface-water-quality data from samples collected at station 333150090530400, an unnamed tributary to Clear Creek near Napanee, MS.-Continued

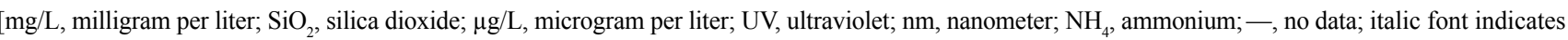
composite sample; see fig. 1 for location]

\begin{tabular}{|c|c|c|c|c|c|c|c|c|c|c|c|c|c|}
\hline Date & $\begin{array}{c}\text { Ortho- } \\
\text { phosphate, } \\
\text { water, } \\
\text { filtered, } \\
\text { mg/L as } \\
\text { phosphorus }\end{array}$ & $\begin{array}{c}\begin{array}{c}\text { Organic } \\
\text { carbon, } \\
\text { water } \\
\text { fitered, } \\
\text { mgred. }\end{array} \\
\end{array}$ & $\begin{array}{c}\text { Inorganic } \\
\text { carbon, } \\
\text { suspended } \\
\text { sediment, } \\
\text { total, } \\
\text { mgl/ }\end{array}$ & $\begin{array}{c}\text { Organic } \\
\text { carbon, } \\
\text { suspended } \\
\text { sediment, } \\
\text { total, } \\
\text { mgl/L }\end{array}$ & $\begin{array}{c}\text { Carbon } \\
\text { (inorganic } \\
\text { plus } \\
\text { oryanicl, } \\
\text { suspended } \\
\text { sediment, } \\
\text { total, } \\
\text { mg/L }\end{array}$ & $\begin{array}{c}\text { Hardness, } \\
\text { water, } \\
\text { mgllas } \\
\text { calcium } \\
\text { carbonate }\end{array}$ & $\begin{array}{c}\text { Noncarbonate } \\
\text { hardness, } \\
\text { water, } \\
\text { filtered, lab, } \\
\text { mol/ as } \\
\text { calcium } \\
\text { carbonate }\end{array}$ & $\begin{array}{c}\text { Calcium, } \\
\text { water, } \\
\text { filtered, } \\
\text { mgl/ }\end{array}$ & $\begin{array}{c}\text { Magnesium, } \\
\text { water, } \\
\text { filtered, } \\
\text { mg/L/L }\end{array}$ & $\begin{array}{c}\text { Sodium, } \\
\text { water, } \\
\text { fitered, } \\
\text { mgl/ }\end{array}$ & $\begin{array}{c}\text { Sodium } \\
\text { adsorproition } \\
\text { ratio, } \\
\text { water, } \\
\text { number }\end{array}$ & $\begin{array}{c}\text { Sodium } \\
\text { fraction of } \\
\text { cations, } \\
\text { water, } \\
\text { percent in } \\
\text { equivalents } \\
\text { of major } \\
\text { cations }\end{array}$ & $\begin{array}{c}\text { Potassium } \\
\text { water, } \\
\text { filtered, } \\
\text { mgl/ }\end{array}$ \\
\hline 4/14/2007 & 0.027 & 5.7 & 1.30 & 33.0 & 34.3 & 27 & 17 & 7.24 & 2.25 & 9.20 & 0.77 & 40 & 1.87 \\
\hline $4 / 141 / 2007$ & 0.012 & 7.7 & 0.39 & 10.9 & 11.3 & 42 & 28 & 11.0 & 3.54 & 13.4 & 0.90 & 39 & 2.47 \\
\hline $4 / 26 / 2007$ & E0.005 & 9.1 & $<0.12$ & 4.92 & 4.92 & 62 & 11 & 16.1 & 5.24 & 18.4 & 1.02 & 38 & 2.96 \\
\hline $5 / 4 / 2007$ & 0.018 & 6.3 & 0.66 & 30.6 & 31.2 & 51 & 22 & 13.0 & 4.39 & 18.9 & 1.16 & 43 & 2.40 \\
\hline $5 / 22 / 2007$ & 0.037 & 5.3 & $<0.12$ & 0.54 & 0.54 & 270 & 20 & 71.5 & 23.3 & 68.4 & 1.80 & 35 & 5.44 \\
\hline $6 / 9 / 2007$ & 0.025 & 12.1 & $<0.12$ & 0.61 & 0.61 & 220 & - & 56.0 & 19.5 & 68.2 & 2.00 & 40 & 5.03 \\
\hline 6/19/2007 & 0.049 & 11.9 & 0.29 & 6.61 & 6.90 & 170 & - & 40.7 & 17.3 & 63.8 & 2.11 & 44 & 3.04 \\
\hline $6 / 19 / 2007$ & 0.054 & 6.0 & $<0.12$ & 5.30 & 5.39 & 30 & - & 7.34 & 2.72 & 15.9 & 1.27 & 52 & 2.00 \\
\hline $6 / 191 / 2007$ & 0.041 & 7.5 & $<0.12$ & 4.88 & 4.88 & 48 & - & 11.8 & 4.49 & 21.6 & 1.36 & 48 & 2.27 \\
\hline $6 / 19 / 2007$ & 0.043 & 8.0 & $<0.12$ & 3.56 & 3.56 & 70 & - & 17.2 & 6.53 & 27.3 & 1.42 & 45 & 2.31 \\
\hline $6 / 191 / 2007$ & 0.043 & 10.9 & $<0.12$ & 6.57 & 6.61 & 59 & - & 14.6 & 5.41 & 26.2 & 1.49 & 48 & 2.38 \\
\hline $6 / 19 / 2007$ & 0.035 & 9.0 & $<0.12$ & 5.28 & 5.28 & 60 & - & 14.7 & 5.55 & 26.9 & 1.51 & 48 & 2.35 \\
\hline $6 / 19 / 2007$ & 0.028 & 9.6 & 0.13 & 2.66 & 2.79 & 68 & - & 17.1 & 6.15 & 28.1 & 1.48 & 46 & 2.32 \\
\hline 6/20/2007 & 0.028 & 9.7 & $<0.12$ & 3.61 & 3.61 & 82 & - & 20.6 & 7.36 & 31.0 & 1.49 & 44 & 2.28 \\
\hline $6 / 20 / 2007$ & 0.022 & 6.0 & $<0.12$ & 1.87 & 1.89 & 100 & 22 & 25.8 & 9.06 & 34.4 & 1.48 & 42 & 2.59 \\
\hline $6 / 21 / 2007$ & 0.015 & 5.4 & $<0.12$ & 1.08 & 1.08 & 130 & - & 34.4 & 11.5 & 42.9 & 1.62 & 41 & 2.79 \\
\hline $6 / 21 / 2007$ & 0.010 & 5.8 & $<0.12$ & 1.20 & 1.23 & 140 & - & 36.5 & 12.1 & 45.1 & 1.65 & 40 & 2.90 \\
\hline 6/26/2007 & 0.039 & 5.3 & 0.25 & 5.58 & 5.84 & 84 & - & 21.9 & 7.08 & 22.7 & 1.08 & 36 & 2.06 \\
\hline 6/27/2007 & 0.035 & 4.2 & $<0.12$ & 1.33 & 1.38 & 120 & - & 32.6 & 10.4 & 29.8 & 1.16 & 34 & 1.64 \\
\hline $77 / 72007$ & 0.084 & 5.1 & 0.35 & 57.3 & 57.6 & 93 & - & 24.4 & 7.91 & 24.2 & 1.09 & 35 & 1.94 \\
\hline $77 / 72007$ & 0.040 & 3.9 & $<0.12$ & 3.32 & 3.38 & 25 & - & 6.47 & 2.23 & 8.81 & 0.76 & 41 & 1.51 \\
\hline 7/7/2007 & 0.030 & 3.6 & $<0.12$ & 2.42 & 2.47 & 25 & - & 6.47 & 2.22 & 8.65 & 0.75 & 41 & 1.53 \\
\hline $7 / 7 / 2007$ & 0.031 & 3.5 & $<0.12$ & 2.13 & 2.16 & 46 & - & 12.0 & 3.86 & 12.6 & 0.81 & 36 & 1.71 \\
\hline $7 / 9 / 2007$ & 0.022 & 4.0 & $<0.12$ & 2.55 & 2.60 & 90 & - & 24.9 & 6.84 & 20.6 & 0.94 & 33 & 1.73 \\
\hline $7 / 10 / 2007$ & 0.024 & 4.5 & $<0.12$ & 1.49 & 1.54 & 85 & - & 23.3 & 6.41 & 18.8 & 0.89 & 32 & 1.85 \\
\hline $8 / 2 / 2007$ & 0.048 & 5.6 & $<0.12$ & 1.52 & 1.56 & 210 & - & 54.7 & 18.4 & 50.7 & 1.51 & 33 & 6.00 \\
\hline $8 / 2 / 2007$ & 0.050 & 5.5 & $<0.12$ & 1.52 & 1.53 & 220 & - & 55.8 & 18.7 & 51.7 & 1.53 & 33 & 5.96 \\
\hline $8 / 2 / 2007$ & 0.038 & 4.9 & $<0.12$ & 0.52 & 0.54 & 240 & - & 62.3 & 19.6 & 64.7 & 1.83 & 37 & 5.62 \\
\hline $8 / 31 / 2007$ & 0.203 & 12.5 & $<0.12$ & 2.21 & 2.21 & 150 & 0 & 38.0 & 14.4 & 52.3 & 1.83 & 41 & 7.54 \\
\hline 3/42008 & 0.067 & 17.9 & Е0.08 & 7.29 & 7.37 & 11 & - & 2.85 & 0.981 & 4.06 & 0.53 & 40 & 1.72 \\
\hline 3/4/2008 & 0.023 & 4.6 & Е0.04 & 6.55 & 5.59 & 12 & - & 3.05 & 1.02 & 4.21 & 0.53 & 40 & 1.59 \\
\hline 3/5/2008 & 0.058 & 6.2 & $<0.04$ & 4.71 & 4.71 & 21 & - & 5.58 & 1.73 & 4.33 & 0.41 & 28 & 2.08 \\
\hline $3 / 5 / 2008$ & 0.039 & 8.2 & $<0.04$ & 3.18 & 3.18 & 29 & - & 7.73 & 2.29 & 4.53 & 0.37 & 24 & 2.33 \\
\hline $4 / 1 / 2008$ & 0.028 & 6.6 & 0.73 & 30.1 & 30.8 & 29 & 0 & 7.39 & 2.51 & 10.5 & 0.85 & 42 & 2.34 \\
\hline 4/1/2008 & 0.054 & 5.7 & 0.19 & 8.51 & 8.70 & 13 & - & 3.37 & 1.15 & 6.59 & 0.79 & 48 & 1.81 \\
\hline
\end{tabular}


Table 5. Surface-water-quality data from samples collected at station 333150090530400, an unnamed tributary to Clear Creek near Napanee, MS.-Continued

mg/L, milligram per liter, $\mathrm{Si}_{2}$, silica dioxide; $\mu \mathrm{g} / \mathrm{L}$, microgram per liter; $\mathrm{UV}$, ultraviolet, $\mathrm{nm}$, nanometer, $\mathrm{NH}_{4}$, ammonium; - , no data; italic font indicates composite sample; see fig. 1 for location]

\begin{tabular}{|c|c|c|c|c|c|c|c|c|c|c|c|c|c|}
\hline Date & $\begin{array}{l}\text { Chloride, } \\
\text { water, } \\
\text { filtered, } \\
\text { mgl/l }\end{array}$ & $\begin{array}{l}\text { Sulfate, } \\
\text { water, } \\
\text { fititerd, } \\
\text { mg/L }\end{array}$ & $\begin{array}{l}\text { Fluoride, } \\
\text { water, } \\
\text { filtered, } \\
\text { mg/L }\end{array}$ & $\begin{array}{c}\text { Silica, } \\
\text { water, } \\
\text { filtered, } \\
\text { mgl/2 } \\
\text { as sio }\end{array}$ & $\begin{array}{c}\text { Iron, } \\
\text { water, } \\
\text { filtered, } \\
\text { rygl/ }\end{array}$ & $\begin{array}{c}\text { Manganese, } \\
\text { water, } \\
\text { filtered, } \\
\text { pg/L }\end{array}$ & $\begin{array}{l}\text { Alkalinity, } \\
\text { water, } \\
\text { filtered, } \\
\text { fixed end- } \\
\text { point } \\
\text { po 4.5) } \\
\text { itratition, } \\
\text { laboratory, } \\
\text { myllas } \\
\text { calcium } \\
\text { carbonate }\end{array}$ & $\begin{array}{c}\text { Particulate } \\
\text { nitrogen, } \\
\text { suspended } \\
\text { in water, } \\
\text { mg/L }\end{array}$ & 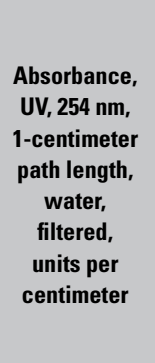 & $\begin{array}{c}\text { Amino- } \\
\text { methyl- } \\
\text { phosphonic } \\
\text { acid, water, } \\
\text { filtered, } \\
\text { recoverable, } \\
\text { pg/L }\end{array}$ & $\begin{array}{c}\text { Glufosinate, } \\
\text { water, } \\
\text { filtered, } \\
\text { recoverable, } \\
\text { pg/L }\end{array}$ & $\begin{array}{l}\text { Giyphosate, } \\
\text { water, } \\
\text { fititerd, } \\
\text { recoverable, } \\
\text { pgl/ }\end{array}$ & $\begin{array}{c}\text { Total } \\
\text { nitrogen } \\
\text { (nitrate + } \\
\text { nitrite + } \\
\text { ammonia } \\
\text { organic-N), } \\
\text { water, } \\
\text { unfitered, } \\
\text { analytically } \\
\text { determined, } \\
\text { mg/l/ }\end{array}$ \\
\hline 14/2007 & 2.22 & 16.5 & 0.25 & 5.42 & E5 & 11.8 & 10 & 4.71 & 0.132 & 2.6 & $<0.02$ & 11 & 9.34 \\
\hline $144 / 2007$ & 5.08 & 22.0 & 0.23 & 6.87 & E6 & 19.3 & 14 & 1.88 & 0.162 & 1.9 & $<0.02$ & 4.1 & 11.4 \\
\hline 62007 & 5.14 & 30.8 & 0.31 & 5.91 & E3 & 0.5 & 51 & 0.62 & 0.160 & 2.0 & $<0.02$ & 1.2 & 5.46 \\
\hline $5 / 4 / 2007$ & 6.44 & 28.0 & 0.31 & 8.06 & 6 & 0.5 & 29 & 5.74 & 0.141 & 1.8 & $<0.02$ & 2.6 & 11.4 \\
\hline $5 / 22 / 20007$ & 29.7 & 117 & 1.35 & 17.2 & $E 3$ & 0.3 & 254 & 0.09 & 0.130 & 8.9 & $<0.02$ & 22 & 4.30 \\
\hline 6992007 & 26.7 & 110 & 0.55 & 13.6 & $E 5$ & 0.3 & 232 & 0.11 & 0.146 & 2.8 & $<0.02$ & 7.7 & 13.8 \\
\hline 9/2007 & 22.5 & 88.1 & 0.56 & 13.0 & E5 & 0.4 & 186 & 1.05 & 0.142 & 3.4 & $<0.02$ & 13 & 16.5 \\
\hline 9/2007 & 5.74 & 21.1 & 0.36 & 4.96 & 6 & E0.2 & 37 & 0.93 & 0.119 & 2.0 & $<0.02$ & 1.9 & 5.93 \\
\hline 6/19/2007 & 6.91 & 28.3 & 0.37 & 5.97 & 9 & 0.3 & 56 & 0.88 & 0.121 & 2.2 & $<0.02$ & 2.4 & 7.99 \\
\hline 6/19/2007 & 8.48 & 35.0 & 0.45 & 7.76 & 9 & 0.4 & 82 & 0.53 & 0.117 & 2.1 & $<0.02$ & 2.9 & 9.83 \\
\hline 9/2007 & 9.19 & 30.7 & 0.36 & 7.78 & 10 & 0.2 & 76 & 1.04 & 0.131 & 0.64 & $<0.02$ & 1.2 & 10.4 \\
\hline 19/2007 & 8.71 & 32.2 & 0.39 & 7.68 & E5 & 0.2 & 74 & 0.71 & 0.125 & 1.3 & $<0.02$ & 1.2 & 9.39 \\
\hline 9/2007 & 8.83 & 35.2 & 0.72 & 8.16 & E5 & 0.4 & 86 & 0.45 & 0.121 & 1.2 & $<0.02$ & 1.4 & 10.7 \\
\hline 0/2007 & 9.73 & 39.2 & 0.47 & 9.15 & 7 & 0.5 & 102 & 0.61 & 0.124 & 1.4 & $<0.02$ & 1.8 & 10.7 \\
\hline D/2007 & 11.2 & 43.6 & 0.82 & 10.2 & E4 & 0.3 & 80 & 0.35 & 0.098 & 1.4 & $<0.02$ & 1.6 & 11.1 \\
\hline $6 / 21 / 2007$ & 12.4 & 51.1 & 0.84 & 11.7 & E3 & 0.3 & 146 & 0.16 & 0.117 & 1.2 & $<0.02$ & 1.1 & 13.0 \\
\hline $6 / 21 / 2007$ & 12.3 & 51.9 & 0.88 & 12.5 & 7 & 0.3 & 168 & 0.25 & 0.106 & 1.0 & $<0.02$ & 1.4 & 10.2 \\
\hline $6 / 26 / 2007$ & 5.21 & 28.2 & 0.62 & 10.0 & $E 5$ & 0.2 & 87 & 0.97 & 0.138 & 4.0 & $<0.02$ & 8.4 & 3.55 \\
\hline 27/2007 & 7.50 & 40.7 & 0.56 & 14.1 & $E 3$ & 0.3 & 134 & 0.19 & 0.108 & 1.9 & $<0.02$ & 3.6 & 2.10 \\
\hline $7 / 72007$ & 8.12 & 27.2 & 0.37 & 15.2 & 37 & 235 & 108 & 9.61 & 0.113 & 0.97 & $<0.02$ & 0.71 & 4.63 \\
\hline $7 / 72007$ & 2.38 & 6.66 & 0.25 & 6.50 & 9 & 0.2 & 34 & 0.56 & 0.095 & 1.2 & $<0.02$ & 1.6 & 1.41 \\
\hline $7 / 722007$ & 2.28 & 5.72 & 0.23 & 5.83 & 11 & 0.3 & 35 & 0.37 & 0.097 & 0.96 & $<0.02$ & 1.2 & 0.91 \\
\hline $7 / 7 / 2007$ & 3.62 & 11.1 & 0.26 & 8.56 & 14 & 0.5 & 59 & 0.39 & 0.106 & 1.2 & $<0.02$ & 1.2 & 0.80 \\
\hline $79 / 22007$ & 6.95 & 19.3 & 0.47 & 13.3 & 9 & 0.2 & 107 & 0.34 & 0.107 & 1.1 & $<0.02$ & 1.1 & 0.90 \\
\hline 7/10/2007 & 6.36 & 16.7 & 0.50 & 13.3 & 14 & 0.3 & 100 & 0.25 & 0.144 & 0.90 & $<0.02$ & 0.71 & 0.69 \\
\hline $82 / 2007$ & 29.4 & 65.0 & 0.62 & 29.8 & 11 & 0.4 & 226 & 0.23 & 0.156 & 0.65 & $<0.02$ & 0.27 & 0.93 \\
\hline 8/22007 & 30.2 & 62.7 & 0.49 & 29.6 & 10 & 0.4 & 228 & 0.19 & 0.153 & 0.73 & $<0.02$ & 0.28 & 0.72 \\
\hline $8 / 2 / 2007$ & 29.5 & 77.7 & 0.53 & 23.3 & 6 & 0.3 & 263 & 0.07 & 0.130 & 0.76 & $<0.02$ & 0.28 & 0.51 \\
\hline $8 / 31 / 2007$ & 22.7 & 77.7 & 0.33 & 11.9 & 36 & 34.4 & 153 & 0.36 & 0.335 & 4.0 & $<0.02$ & 4.6 & 1.33 \\
\hline 3/4/2008 & 1.28 & 3.07 & 0.25 & 3.73 & 11 & 1.7 & 18 & 1.17 & 0.133 & 0.77 & $<0.02$ & 0.24 & 1.18 \\
\hline 2008 & 1.21 & 2.93 & 0.19 & 3.74 & 57 & 0.5 & 19 & 1.16 & 0.115 & 0.79 & $<0.02$ & 0.14 & 1.20 \\
\hline 3/5/2008 & 1.44 & 3.29 & 0.13 & 5.18 & 63 & 0.9 & 29 & 0.81 & 0.178 & 0.49 & $<0.02$ & 0.10 & 1.05 \\
\hline 3/5/2008 & 1.40 & 3.42 & 0.13 & 4.93 & 95 & 3.9 & 36 & 0.40 & 0.238 & 0.48 & $<0.02$ & 0.03 & 1.16 \\
\hline (122008 & 4.51 & 9.80 & 0.24 & 5.64 & 17 & E0.3 & 28 & 4.34 & 0.159 & 2.5 & $<0.02$ & 1.4 & 6.15 \\
\hline & .21 & 6.03 & 0.39 & 20 & 60 & & & 39 & 155 & 2.7 & 0.02 & 3.2 & 2.84 \\
\hline
\end{tabular}

Table 5. Surface-water-quality data from samples collected at station 333150090530400, an unnamed tributary to Clear Creek near Napanee, MS.-Continued

[mg/L, milligram per liter; $\mathrm{SiO}_{O}$, silica dioxide; $\mu \mathrm{g} / \mathrm{L}$, microgram per liter; $\mathrm{UV}$, ultraviolet, nm, nanometer; $\mathrm{NH}_{4}$ a ammonium;-, no data; italic font indicates composite sample; see fig. 1 for location]

\begin{tabular}{|c|c|c|c|c|c|c|c|c|c|c|c|c|c|}
\hline Date & $\begin{array}{l}\text { Specific UV } \\
\text { absotrance, } \\
\text { 254 nu, } \\
\text { water, } \\
\text { filtered }\end{array}$ & $\begin{array}{l}\text { Turbiditity, } \\
\text { water } \\
\text { unfitered, } \\
\text { in } \\
\text { Nephelo- } \\
\text { metric } \\
\text { Turbiditity } \\
\text { Units }\end{array}$ & $\begin{array}{l}\text { Dissolved } \\
\text { solids } \\
\text { dried } \\
\text { at t t880 } \\
\text { degres } \\
\text { Celsius, } \\
\text { water, } \\
\text { filtered, } \\
\text { mg/L/ }\end{array}$ & $\begin{array}{l}\text { Dissolved } \\
\text { solids, } \\
\text { water } \\
\text { fitered, } \\
\text { sum of } \\
\text { consit. } \\
\text { uentst. } \\
\text { mgl/L }\end{array}$ & $\begin{array}{l}\text { Dissolved } \\
\text { solids, } \\
\text { water, } \\
\text { tons } \\
\text { per day }\end{array}$ & $\begin{array}{l}\text { Dissolved } \\
\text { solids, } \\
\text { water } \\
\text { filtered, tons } \\
\text { per acre-foot }\end{array}$ & $\begin{array}{l}\text { Ammonia, } \\
\text { water, } \\
\text { filtered, } \\
\text { mgy/L } \\
\text { as } \mathrm{NH}_{4}\end{array}$ & $\begin{array}{c}\text { Nitrate, } \\
\text { water, } \\
\text { filtered, } \\
\text { mg/L }\end{array}$ & $\begin{array}{c}\text { Nititite, } \\
\text { water, } \\
\text { filtered, } \\
\text { mgl/l }\end{array}$ & $\begin{array}{l}\text { Suspended } \\
\text { sediment } \\
\text { concen- } \\
\text { tration, } \\
\text { mg/L }\end{array}$ & $\begin{array}{c}\text { Suspended } \\
\text { sediment } \\
\text { discharge, } \\
\text { tons } \\
\text { per day }\end{array}$ & $\begin{array}{c}\text { Specific } \\
\text { conduc- } \\
\text { tance, } \\
\text { water, } \\
\text { unifitered, } \\
\text { laboratory, } \\
\text { micro- } \\
\text { siemens } \\
\text { per centi- } \\
\text { meter at } \\
\text { 25 degrees } \\
\text { Celsius }\end{array}$ & $\begin{array}{c}\text { Ratio of } \\
\text { particulate } \\
\text { nitrogen to to } \\
\text { particute } \\
\text { organic } \\
\text { carbon, } \\
\text { number }\end{array}$ \\
\hline 4/4/4/2007 & 2.3 & $>1,000$ & 77 & E75 & 0.01 & 0.10 & 0.100 & 23.4 & 0.123 & 2,790 & 0.53 & 117 & 0.143 \\
\hline 4/14/2007 & 2.1 & $>1,000$ & 130 & EII2 & - & 0.18 & 0.082 & 38.8 & 0.429 & 1,210 & - & 174 & 0.172 \\
\hline $4 / 26 / 2007$ & 1.8 & 650 & 140 & E133 & 0.07 & 0.19 & Е0.014 & 17.3 & 0.130 & 519 & 0.27 & 226 & 0.126 \\
\hline $5 / 4 / 2007$ & 2.3 & - & 130 & 135 & - & 0.18 & 0.077 & 35.7 & 0.171 & 2,500 & - & 215 & 0.188 \\
\hline $5 / 22 / 20007$ & 2.5 & 7.0 & 523 & $E 502$ & - & 0.71 & 0.035 & 7.43 & 5.66 & 78 & - & 803 & 0.160 \\
\hline $619 / 2007$ & 1.2 & 5.0 & 476 & $E 445$ & - & 0.65 & 2.18 & 4.42 & 0.164 & 81 & - & 742 & 0.176 \\
\hline 6/19/2007 & 1.2 & 370 & 390 & E380 & 3.56 & 0.53 & 3.04 & 13.1 & 1.86 & 400 & 3.7 & 636 & 0.159 \\
\hline 6/19/2007 & 2.0 & $>1,000$ & 104 & E89 & 4.76 & 0.14 & 0.734 & 5.73 & 0.371 & 826 & 38 & 157 & 0.175 \\
\hline 6/19/2007 & 1.6 & 520 & 142 & 122 & 6.88 & 0.19 & 1.17 & 4.67 & 0.413 & 364 & 18 & 215 & 0.180 \\
\hline 6/19/2007 & 1.5 & 520 & 168 & 160 & 8.01 & 0.23 & 1.62 & 3.15 & 0.336 & 244 & 12 & 277 & 0.149 \\
\hline 6/19/2007 & 1.2 & 850 & 155 & 148 & 8.69 & 0.21 & 1.74 & 4.06 & 0.300 & 504 & 28 & 260 & 0.158 \\
\hline 6/19/2007 & 1.4 & 490 & 158 & E148 & 8.68 & 0.21 & 1.72 & 3.00 & 0.278 & 343 & 19 & 258 & 0.135 \\
\hline 6/19/2007 & 1.3 & 190 & 177 & E163 & 8.91 & 0.24 & 2.12 & 2.38 & 0.277 & 199 & 10 & 286 & 0.168 \\
\hline 6/20/2007 & 1.3 & 110 & 201 & 186 & 9.76 & 0.27 & 2.30 & 1.91 & 0.272 & 207 & 10 & 325 & 0.170 \\
\hline 6/20/2007 & 1.6 & 130 & 235 & E207 & 6.97 & 0.32 & 3.55 & 6.91 & 8.03 & 140 & 4.2 & 391 & 0.185 \\
\hline $6 / 21 / 2007$ & 2.2 & 38 & 259 & E287 & 5.53 & 0.35 & 5.72 & 5.25 & 15.2 & 109 & 2.3 & 485 & 0.151 \\
\hline 6/21/2007 & 1.8 & 26 & 278 & 300 & - & 0.38 & 6.20 & 5.21 & 9.94 & 84 & - & 510 & 0.207 \\
\hline $6 / 26 / 2007$ & 2.6 & 690 & 164 & E161 & - & 0.22 & $E 0.014$ & 10.6 & 0.072 & 443 & - & 270 & 0.174 \\
\hline $6 / 27 / 2007$ & 2.6 & 61 & 228 & $E 224$ & - & 0.31 & 0.014 & 5.81 & 0.074 & 101 & - & 379 & 0.144 \\
\hline $7 / 7 / 2007$ & 2.2 & - & 190 & 179 & 2.82 & 0.26 & 0.033 & 4.68 & 0.112 & 702 & 10 & 298 & 0.168 \\
\hline $7 / 7 / 2007$ & 2.5 & - & 52 & 58 & 2.80 & 0.07 & 0.046 & 2.86 & 0.040 & 255 & 14 & 94 & 0.170 \\
\hline $7 / 72007$ & 2.7 & - & 63 & 56 & 3.71 & 0.09 & $<0.026$ & 1.40 & 0.056 & 152 & 9.0 & 91 & 0.152 \\
\hline $7 / 7 / 2007$ & 3.1 & 72 & 101 & 90 & - & 0.14 & 0.044 & 0.999 & 0.024 & 73 & - & 153 & 0.185 \\
\hline $79 / 2007$ & 2.7 & 160 & 166 & 159 & 2.78 & 0.23 & 0.060 & 0.781 & 0.268 & 152 & 2.5 & 271 & 0.133 \\
\hline 7/10/2007 & 3.2 & - & 152 & 148 & 2.53 & 0.21 & 0.044 & 0.331 & 0.135 & 95 & 1.6 & 252 & 0.169 \\
\hline 8/2/2007 & 2.8 & 67 & 412 & E392 & 11.1 & 0.56 & Е0.017 & 0.821 & 0.488 & 242 & 6.5 & 644 & 0.151 \\
\hline 82/2007 & 2.8 & 52 & 428 & E393 & 12.7 & 0.58 & Е0.014 & 0.614 & 0.297 & 194 & 5.8 & 653 & 0.122 \\
\hline $8 / 2 / 2007$ & 2.7 & 9.0 & 466 & 442 & - & 0.63 & 0.099 & 0.208 & 0.099 & 113 & - & 730 & 0.128 \\
\hline $8 / 31 / 2007$ & 2.7 & 47 & 352 & 318 & 2.61 & 0.48 & 0.071 & 0.239 & 0.052 & 112 & 0.83 & 539 & 0.164 \\
\hline 3/42008 & 0.7 & 770 & 29 & E30 & 2.51 & 0.04 & Е0.026 & 0.651 & 0.020 & 426 & 37 & 46 & 0.161 \\
\hline 3/4/2008 & 2.5 & 690 & 33 & 30 & 2.70 & 0.05 & $<0.026$ & $<0.615$ & $<0.007$ & 419 & 34 & 52 & 0.178 \\
\hline $3 / 5 / 2008$ & 2.9 & 420 & 44 & 42 & 1.76 & 0.06 & $<0.026$ & 0.648 & 0.025 & 281 & 11 & 69 & 0.172 \\
\hline $3 / 5 / 2008$ & 2.9 & 330 & 59 & 49 & - & 0.08 & $<0.026$ & 0.770 & 0.082 & 221 & - & 84 & 0.125 \\
\hline 4/1/2008 & 2.4 & $>1,000$ & 91 & E73 & 4.03 & 0.12 & 0.031 & 12.4 & 0.092 & 3,170 & 140 & 124 & 0.144 \\
\hline 1/2008 & 2.7 & $>1,000$ & 56 & E43 & 4.98 & 0.08 & 0.016 & 5.93 & 0.159 & 641 & 57 & 67 & 0.163 \\
\hline
\end{tabular}


Table 5. Surface-water-quality data from samples collected at station 333150090530400, an unnamed tributary to Clear Creek near Napanee, MS.-Continued

[mg/L, milligram per liter; $\mathrm{Si}_{2}$, silica dioxide; $\mu \mathrm{g} / \mathrm{L}$, microgram per liter; $\mathrm{UV}$, ultraviolet; $\mathrm{nm}$, nanometer; $\mathrm{NH}_{4}$, ammonium; -, no data; italic font indicates (a)



\begin{tabular}{lccccccccccccc}
\hline $41 / 2008$ & - & - & 80 & - & - & 7.5 & 1.4 & 0.030 & 0.032 & 0.992 & 1.02 & 0.177 & 0.41 \\
$4 / 1 / 2008$ & 31 & 7.29 & 76 & - & - & 7.5 & 1.4 & 0.030 & 0.032 & 0.992 & 1.02 & 0.177 & 0.41
\end{tabular}

$\begin{array}{llllllllllllll}4 / 1 / 2008 & 31 & 7.29 & 76 & - & - & 7.5 & 1.4 & 0.030 & 0.032 & 0.992 & 1.02 & 0.177 & 0.41 \\ 4 & 50 & 530 & 99 & - & - & 72 & 27 & 0.075 & 0.012 & 0.515 & 0.53 & 0.127 & 0.99\end{array}$

$\begin{array}{llllllllllllll}4 / 4 / 2008 & 51 & 8.18 & 48 & - & - & 7.5 & 0.99 & 0.039 & 0.007 & 0.166 & 0.17 & 0.106 & 0.37\end{array}$

$\begin{array}{llllllllllllll}4 \text { 4/5/2008 } & 50 & 8.15 & 48 & - & - & 7.4 & 0.81 & 0.035 & 0.005 & 0.126 & 0.13 & 0.099 & 0.28\end{array}$

$\begin{array}{llllllllllllll}4 / 5 / 2008 & 39 & 7.75 & - & - & - & 7.4 & 0.99 & 0.028 & 0.005 & 0.118 & 0.12 & 0.102 & 0.30 \\ 4 / 5 / 2008 & - & - & 62 & - & - & 7.3 & 1.0 & 0.066 & 0.007 & 0.129 & 0.14 & 0.187 & 0.36\end{array}$

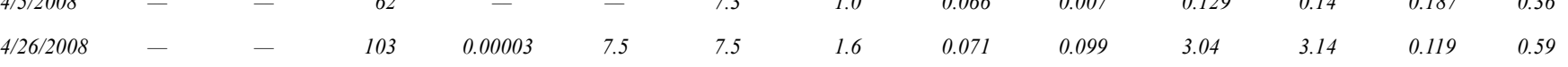

$\begin{array}{llllllllllllll}4 / 26 / 2008 & - & - & 119 & 0.00016 & 6.8 & 8.0 & 1.7 & 0.069 & 0.134 & 3.51 & 3.64 & 0.097 & 0.48\end{array}$

$\begin{array}{llllllllllllll}5 / 222008 & 0.39 & 4.55 & 112 & 0.00010 & 7.0 & 7.3 & 2.3 & 0.057 & 0.020 & 1.43 & 1.45 & 0.072 & 0.96\end{array}$

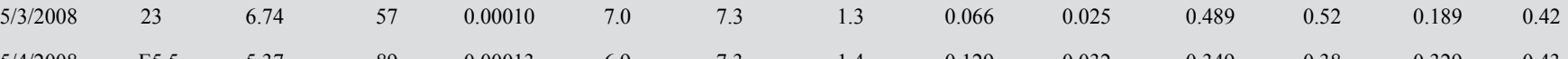

$\begin{array}{llllllllllllll}5 / 4 / 2008 & \mathrm{E} 1.3 & 4.77 & 89 & 0.00013 & 6.9 & 7.5 & 1.4 & 0.147 & 0.017 & 0.115 & 0.13 & 0.359 & 0.41\end{array}$

$\begin{array}{llllllllllllll}5 / 5 / 2008 & 0.43 & 4.56 & 108 & 0.00013 & 6.9 & 7.5 & 1.4 & 0.094 & 0.013 & 0.081 & 0.09 & 0.229 & 0.38 \\ & 0.969 & \end{array}$

$\begin{array}{llllllllllllll}5 / 14 / 2008 & 0.70 & 4.64 & 241 & 0.00003 & 7.5 & 7.6 & 1.6 & 0.049 & 0.040 & 4.92 & 4.96 & 0.066 & 0.29 \\ 5 / 152008 & 1.0 & 4.71 & 171 & 0.00003 & 7.6 & 7.4 & 1.0 & 0.095 & 0.049 & 3.40 & 3.45 & 0.062 & 0.41\end{array}$

$\begin{array}{llllllllllllll}5 / 15 / 2008 & 41 & 7.82 & 62 & 0.00001 & 7.9 & 7.4 & 0.87 & 0.050 & 0.016 & 1.15 & 1.16 & 0.085 & 0.33\end{array}$

$\begin{array}{llllllllllllll}5 / 15 / 2008 & 39 & 7.73 & 68 & 0.00013 & 6.9 & 7.4 & 0.99 & 0.056 & 0.016 & 0.978 & 0.99 & 0.097 & 0.30\end{array}$

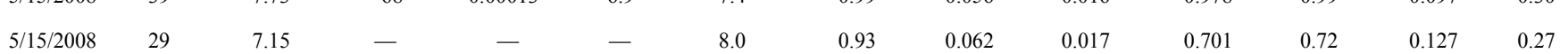

$\begin{array}{llllllllllllll}5 / 16 / 2008 & - & 6.89 & 72 & 0.00010 & 7.0 & 7.3 & 0.47 & 0.054 & 0.015 & 0.614 & 0.63 & 0.106 & 0.25 \\ 5 / 162008 & - & - & 92 & 0.00020 & 6.7 & 7.5 & 1.1 & 0.209 & 0.023 & 0.280 & 0.30 & 0.326 & 0.32\end{array}$



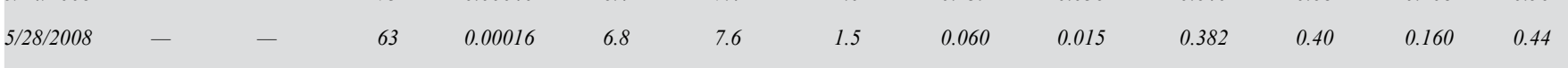

$\begin{array}{llllllllllllll}7 / 15 / 2008 & 0.90 & 4.68 & 792 & - & - & 8.3 & 0.96 & 0.207 & 0.070 & 1.59 & 1.66 & 0.239 & 0.153\end{array}$

$\begin{array}{llllllllllllll}7 / 1622008 & 5.6 & 5.38 & - & - & - & 8.3 & 0.45 & 0.063 & 0.017 & 0.297 & 0.31 & 0.243 & 0.124\end{array}$

$\begin{array}{llllllllllllll}7 / 22 / 2008 & 1.9 & 4.87 & 420 & 0.00020 & 6.7 & 7.7 & \mathrm{E} 1.7 & \mathrm{E} 0.015 & 0.044 & 1.82 & 1.87 & 0.135 & 0.53 \\ 7 / 23 / 2008 & 18 & 6.40 & 361 & 0.00032 & 6.5 & 7.5 & - & <0.020 & 0.098 & 2.28 & 2.38 & 0.143 & 0.316\end{array}$

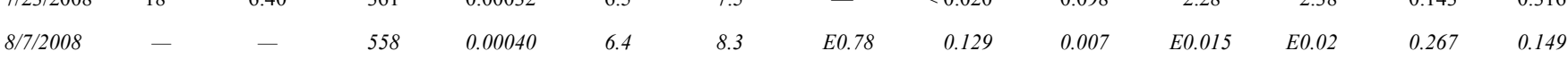

$\begin{array}{llllllllllllll}8 / 192008 & 9.2 & 5.73 & 340 & 0.00004 & 7.4 & 7.7 & \text { E. } 0.94 & \text { E0.017 } & 0.008 & 0.459 & 0.47 & 0.199 & 0.28\end{array}$

$\begin{array}{llllllllllllll}8 / 20 / 2008 & - & - & 78 & 0.00003 & 7.6 & 7.0 & 1.1 & 0.021 & 0.006 & 0.348 & 0.35 & 0.436 & 0.37 \\ 9 / 2 / 2008 & - & - & 125 & 0.00025 & 6.6 & 7.2 & 1.5 & 0.075 & 0.018 & 1.77 & 1.79 & 0.316 & 0.33\end{array}$

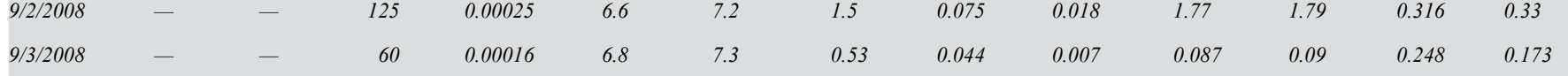

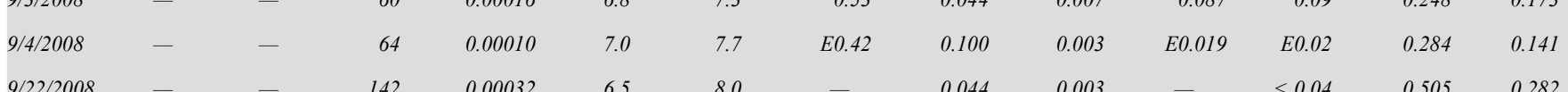



Table 5. Surface-water-quality data from samples collected at station 333150090530400, an unnamed tributary to Clear Creek near Napanee, MS.-Continued

$\left[\mathrm{mg} / \mathrm{L}\right.$, milligram per liter, $\mathrm{SiO}_{2}$, silica dioxide; $\mu \mathrm{g} / \mathrm{L}$, microgram per liter; $\mathrm{UV}$, ultraviolet, $\mathrm{nm}$, nanometer; $\mathrm{NH}_{4}$, ammonium; - , no data; italic font indicates

\begin{tabular}{|c|c|c|c|c|c|c|c|c|c|c|c|c|c|}
\hline Date & $\begin{array}{c}\text { Ortho- } \\
\text { phosphhate, } \\
\text { water, } \\
\text { filtered, } \\
\text { mg/las } \\
\text { phosphorus }\end{array}$ & $\begin{array}{c}\text { Organic } \\
\text { carbon, } \\
\text { water, } \\
\text { fitered, } \\
\text { myll }\end{array}$ & $\begin{array}{c}\text { Inorganic } \\
\text { carbono, } \\
\text { suspended } \\
\text { sediment, } \\
\text { total, } \\
\text { mgl/ }\end{array}$ & $\begin{array}{c}\text { Organic } \\
\text { carbon, } \\
\text { suspended } \\
\text { sediment, } \\
\text { total, } \\
\text { mgl/L }\end{array}$ & $\begin{array}{c}\text { Carbon } \\
\text { linorganic } \\
\text { oplus } \\
\text { organicl, } \\
\text { suspended } \\
\text { sedinent, } \\
\text { totat, } \\
\text { mgl/ } \\
\text { mgl }\end{array}$ & $\begin{array}{c}\text { Hardness, } \\
\text { water, } \\
\text { mgll as } \\
\text { calcium } \\
\text { carbonate }\end{array}$ & $\begin{array}{c}\text { Noncarbonate } \\
\text { hardness, } \\
\text { water, } \\
\text { filtered, lab, } \\
\text { mol/ as, } \\
\text { calcium } \\
\text { carbonate }\end{array}$ & $\begin{array}{c}\text { Calcium, } \\
\text { water, } \\
\text { fitered, } \\
\text { mg/h }\end{array}$ & $\begin{array}{c}\text { Magnesium, } \\
\text { water, } \\
\text { filtered, } \\
\text { mg/l/ }\end{array}$ & $\begin{array}{c}\text { Sodium, } \\
\text { water, } \\
\text { filitered, } \\
\text { mgl/, }\end{array}$ & $\begin{array}{c}\text { Sodium } \\
\text { adsorption } \\
\text { ratio, } \\
\text { water. } \\
\text { number }\end{array}$ & $\begin{array}{c}\text { Sodium } \\
\text { fraction of } \\
\text { cations, } \\
\text { water, } \\
\text { percent in } \\
\text { equivalents } \\
\text { of major } \\
\text { cations }\end{array}$ & $\begin{array}{c}\text { Potassium, } \\
\text { water, } \\
\text { filtered, } \\
\text { mgl/ }\end{array}$ \\
\hline $4 / 1 / 2008$ & 0.058 & 7.4 & 0.13 & 7.87 & 7.99 & 19 & - & 5.02 & 1.66 & 7.54 & 0.75 & 43 & 2.00 \\
\hline 4/1/2008 & 0.058 & - & 0.13 & 7.87 & 7.99 & 19 & - & 5.02 & 1.66 & 7.54 & 0.75 & 43 & 2.00 \\
\hline 4/4/2008 & 0.041 & 7.1 & 0.25 & 18.2 & 18.4 & 22 & - & 5.61 & 1.84 & 8.58 & 0.80 & 43 & 2.37 \\
\hline 4/4/2008 & 0.035 & 5.8 & $<0.04$ & 6.89 & 6.89 & 10 & - & 2.44 & 0.838 & 4.85 & 0.68 & 48 & 1.40 \\
\hline $4 / 5 / 2008$ & 0.032 & 4.3 & $<0.04$ & 4.34 & 4.34 & 10 & - & 2.63 & 0.870 & 4.73 & 0.65 & 46 & 1.38 \\
\hline $4 / 5 / 2008$ & 0.033 & 7.3 & 0.13 & 7.95 & 8.08 & 14 & - & 3.60 & 1.18 & 5.20 & 0.61 & 42 & 1.59 \\
\hline $4 / 5 / 2008$ & 0.061 & 3.7 & $<0.04$ & 4.92 & 4.92 & 17 & - & 4.37 & 1.37 & 5.00 & 0.54 & 37 & 1.84 \\
\hline $4 / 2612008$ & 0.039 & 8.2 & $E 0.10$ & 14.7 & 14.8 & 24 & 2 & 6.19 & 2.17 & 9.13 & 0.80 & 42 & 2.17 \\
\hline $4 / 2612008$ & 0.032 & 9.6 & 0.33 & 8.35 & 8.68 & 29 & 7 & 7.44 & 2.48 & 9.22 & 0.75 & 39 & 2.31 \\
\hline $5 / 2 / 2008$ & 0.023 & 7.5 & 0.49 & 14.0 & 14.5 & 28 & - & 7.14 & 2.39 & 9.39 & 0.78 & 40 & 2.26 \\
\hline $5 / 3 / 2008$ & 0.061 & 6.0 & 0.21 & 7.71 & 7.92 & 11 & - & 2.86 & 0.934 & 5.23 & 0.69 & 47 & 1.50 \\
\hline $5 / 4 / 2008$ & 0.107 & 10.9 & Е0.03 & 5.41 & 5.44 & 26 & - & 6.90 & 2.11 & 4.58 & 0.39 & 25 & 2.72 \\
\hline $5 / 4 / 2008$ & 0.117 & 11.9 & $<0.04$ & 3.97 & 3.97 & 31 & - & 8.21 & 2.55 & 5.11 & 0.40 & 24 & 3.08 \\
\hline $5 / 5 / 2008$ & 0.075 & 12.2 & Е0.10 & 4.53 & 4.63 & 36 & - & 9.44 & 3.00 & 5.32 & 0.39 & 23 & 2.94 \\
\hline $5 / 1442000$ & 0.022 & 10.8 & $<0.04$ & 4.43 & 4.43 & 69 & 15 & 18.0 & 5.82 & 18.6 & 0.97 & 35 & 3.59 \\
\hline $5 / 15 / 2008$ & 0.020 & 10.6 & $<0.04$ & 11.1 & 11.1 & 54 & 12 & 14.1 & 4.59 & 12.9 & 0.76 & 33 & 3.14 \\
\hline $5 / 15 / 2000$ & 0.028 & 4.5 & $<0.04$ & 7.99 & 7.99 & 14 & - & 3.56 & 1.29 & 6.21 & 0.72 & 45 & 1.61 \\
\hline $5 / 15 / 2000$ & 0.032 & 7.2 & 0.16 & 4.08 & 4.24 & 14 & - & 3.62 & 1.27 & 6.45 & 0.74 & 46 & 1.64 \\
\hline $5 / 15 / 2000$ & 0.041 & 5.7 & 0.63 & 6.38 & 7.01 & 14 & - & 3.45 & 1.21 & 5.42 & 0.64 & 43 & 1.72 \\
\hline $5 / 16 / 2000$ & 0.035 & 5.8 & $<0.04$ & 5.04 & 5.04 & 16 & - & 4.23 & 1.44 & 6.27 & 0.67 & 42 & 1.77 \\
\hline $5 / / 66 / 2008$ & 0.106 & 10.0 & $<0.04$ & 5.67 & 5.67 & 28 & - & 7.42 & 2.34 & 5.21 & 0.43 & 26 & 3.03 \\
\hline $5 / 242008$ & 0.068 & 7.0 & $<0.04$ & 17.4 & 17.4 & 21 & - & 5.35 & 1.77 & 4.22 & 0.40 & 28 & 2.28 \\
\hline $5 / 28 / 2008$ & 0.052 & 6.9 & $E 0.07$ & 11.3 & 11.4 & 17 & - & 4.41 & 1.53 & 4.76 & 0.50 & 34 & 1.95 \\
\hline $7 / 15 / 2008$ & 0.078 & 9.1 & $<0.04$ & 1.28 & 1.29 & 260 & 14 & 67.5 & 21.9 & 66.4 & 1.80 & 35 & 5.92 \\
\hline $7 / 1620008$ & 0.079 & 7.8 & $<0.04$ & 0.56 & 0.57 & 310 & 12 & 80.5 & 25.4 & 72.5 & 1.81 & 34 & 5.70 \\
\hline 7/222/2000 & 0.044 & 7.1 & 0.32 & 8.20 & 8.52 & 130 & - & 34.5 & 11.2 & 41.7 & 1.58 & 40 & 4.15 \\
\hline $7 / 23212008$ & 0.047 & 9.5 & $<0.04$ & 3.76 & 3.76 & 110 & - & 28.9 & 9.48 & 38.5 & 1.59 & 42 & 4.64 \\
\hline $87 / 2008$ & 0.087 & - & $<0.04$ & 0.92 & 0.92 & 150 & - & 36.2 & 15.0 & 59.6 & 2.10 & 45 & 4.59 \\
\hline $8 / 19 / 2008$ & 0.065 & 6.1 & $<0.04$ & 3.96 & 3.96 & 86 & - & 22.3 & 7.39 & 35.6 & 1.67 & 46 & 5.05 \\
\hline $8 / 20 / 2008$ & 0.142 & 6.9 & $<0.04$ & 5.12 & 5.12 & 18 & - & 4.62 & 1.62 & 8.16 & 0.83 & 45 & 2.88 \\
\hline 9/2/20008 & 0.103 & 10.1 & $<0.04$ & 6.43 & 6.43 & 30 & - & 7.75 & 2.66 & 12.2 & 0.96 & 44 & 3.05 \\
\hline $9 / 3 / 2008$ & 0.081 & 4.8 & $<0.04$ & 2.70 & 2.70 & 14 & - & 3.73 & 1.25 & 5.97 & 0.68 & 43 & 2.10 \\
\hline $9 / 4 / 2008$ & 0.093 & 4.7 & $<0.04$ & 0.85 & 0.85 & 19 & - & 4.83 & 1.60 & 5.02 & 0.51 & 33 & 2.55 \\
\hline $9 / 22 / 2008$ & 0.165 & 9.6 & $E 0.03$ & 1.20 & 1.24 & 54 & - & 13.2 & 5.20 & 9.07 & 0.54 & 26 & 1.09 \\
\hline
\end{tabular}


Table 5. Surface-water-data results from samples collected at station 333150090530400, an unnamed tributary to Clear Creek near Napanee, MS.-Continued

$\left[\mathrm{mg} / \mathrm{L}\right.$, milligram per liter, $\mathrm{SiO}_{2}$, silica dioxide; $\mu \mathrm{g} / \mathrm{L}$, microgram per liter, $\mathrm{UV}$, ultraviolet; $\mathrm{nm}$, nanometer; $\mathrm{NH}_{4}$, ammonium; - , no data; italic font indicates

\begin{tabular}{|c|c|c|c|c|c|c|c|c|c|c|c|c|c|}
\hline Date & $\begin{array}{l}\text { Chloride, } \\
\text { water, } \\
\text { filtered, } \\
\text { mgl/ }\end{array}$ & $\begin{array}{c}\text { Sulfate, } \\
\text { water, } \\
\text { filtered, } \\
\mathrm{mg} / \mathrm{L}\end{array}$ & $\begin{array}{l}\text { Fluoride, } \\
\text { water, } \\
\text { fitered, } \\
\text { mgl/h }\end{array}$ & $\begin{array}{c}\text { Silica, } \\
\text { water, } \\
\text { filtered, } \\
\text { malt } \\
\text { as Silo }\end{array}$ & $\begin{array}{c}\text { Iron, } \\
\text { water, } \\
\text { filtered, } \\
\mathrm{gg} / \mathrm{h}\end{array}$ & $\begin{array}{l}\text { Manganese, } \\
\text { water, } \\
\text { filtered, } \\
\boldsymbol{\mu g} / / \mathrm{L}\end{array}$ & $\begin{array}{l}\text { Alkalinity, } \\
\text { water, } \\
\text { filtered, } \\
\text { fixed end- } \\
\text { point } \\
\text { po 4.5) } \\
\text { itration, } \\
\text { laboratory, } \\
\text { myllas } \\
\text { calcium } \\
\text { carbonate }\end{array}$ & $\begin{array}{c}\text { Particulate } \\
\text { nitrogen, } \\
\text { suspended } \\
\text { in water, } \\
\text { mg/L } \\
\end{array}$ &  & $\begin{array}{c}\text { Amino- } \\
\text { methyl- } \\
\text { phosphhonic } \\
\text { acid, water, } \\
\text { iftered, } \\
\text { recoverable, } \\
\text { pgl/ }\end{array}$ & $\begin{array}{l}\text { Glutosinate, } \\
\text { water. } \\
\text { fitherd, } \\
\text { recoverable, } \\
\text { yg/L }\end{array}$ & $\begin{array}{l}\text { Glyphosate, } \\
\text { water, } \\
\text { fitered, } \\
\text { recoverable, } \\
\text { pg/h }\end{array}$ & $\begin{array}{c}\text { Total } \\
\text { nitrogen } \\
\text { (nitrate + } \\
\text { nitrite + } \\
\text { ammonia } \\
\text { organic-N), } \\
\text { water, } \\
\text { unfitered, } \\
\text { analytically } \\
\text { determined, } \\
\text { mg/l/ }\end{array}$ \\
\hline 1/1/2008 & 2.44 & 7.64 & 0.26 & 6.62 & 26 & 0.5 & 22 & 1.19 & 0.199 & 2.5 & $<0.02$ & 2.3 & 2.43 \\
\hline 2008 & 2.44 & 7.64 & 0.26 & 6.62 & 26 & 0.5 & 22 & 1.19 & - & - & - & - & 2.43 \\
\hline 2008 & 4.95 & 13.8 & 0.16 & 7.24 & 50 & 0.6 & 22 & 2.48 & 0.188 & 0.27 & $<0.02$ & 0.34 & 3.28 \\
\hline 2008 & 1.97 & 5.30 & 0.15 & 4.49 & 119 & 1.7 & 15 & 0.93 & 0.165 & 1.5 & $<0.02$ & 0.72 & 1.21 \\
\hline 2008 & 1.57 & 4.12 & 0.15 & 4.32 & 61 & 0.6 & 17 & 0.60 & 0.099 & 1.8 & $<0.02$ & 1.5 & 0.97 \\
\hline 2008 & 1.58 & 4.76 & 0.18 & 5.20 & 95 & 0.8 & 21 & 1.12 & 0.139 & 2.0 & $<0.02$ & 1.2 & 1.14 \\
\hline 2008 & 1.33 & 4.19 & 0.19 & 5.22 & 61 & 2.0 & 24 & 0.83 & 0.090 & 1.9 & $<0.02$ & 0.87 & 1.22 \\
\hline $5 / 2008$ & 2.68 & 8.51 & 0.22 & 7.44 & 16 & 0.6 & 23 & 2.62 & 0.201 & 1.7 & $<0.02$ & 0.58 & 4.79 \\
\hline 6/2008 & 3.03 & 11.3 & 0.17 & 9.53 & 21 & 0.9 & 22 & 1.46 & 0.218 & 1.3 & $<0.02$ & 0.57 & 5.46 \\
\hline 12/2008 & 3.55 & 12.7 & 0.27 & 7.20 & 42 & 0.8 & 30 & 1.69 & 0.185 & 1.1 & $<0.02$ & 0.20 & 3.85 \\
\hline 2008 & 1.47 & 5.18 & 0.35 & 7.03 & 121 & 0.9 & 17 & 1.26 & 0.177 & 2.0 & $<0.02$ & 0.54 & 1.92 \\
\hline 2008 & 1.98 & 5.75 & 0.26 & 7.41 & 96 & 0.9 & 32 & 0.74 & 0.331 & 1.1 & $<0.02$ & 0.46 & 1.91 \\
\hline 2008 & 2.08 & 6.12 & 0.27 & 8.31 & 139 & 5.4 & 39 & 0.59 & 0.365 & 1.3 & $<0.02$ & 0.51 & 1.66 \\
\hline $5 / 5 / 2008$ & 2.09 & 6.22 & 0.24 & 8.70 & 151 & 22.8 & 45 & 0.71 & 0.366 & 1.6 & $<0.02$ & 0.56 & 1.60 \\
\hline $5 / 14 / 2008$ & 9.14 & 28.0 & 0.25 & 8.36 & 20 & 38.6 & 54 & 0.80 & 0.247 & 2.0 & $<0.02$ & 0.29 & 6.58 \\
\hline $5 / 15 / 2008$ & 5.03 & 21.0 & 0.22 & 9.20 & 13 & 6.8 & 42 & 1.77 & 0.250 & 1.4 & $<0.02$ & 0.22 & 4.57 \\
\hline ;/2008 & 1.60 & 6.88 & 0.16 & 5.50 & 18 & Е 0.3 & 17 & 1.14 & 0.110 & 1.2 & $<0.02$ & 0.34 & 2.08 \\
\hline $5 / 15 / 2008$ & 1.53 & 6.35 & 0.12 & 6.10 & 46 & Е 0.3 & 20 & 0.68 & 0.123 & 1.5 & $<0.02$ & 0.41 & 2.04 \\
\hline $5 / 15 / 2008$ & 1.16 & 5.00 & 0.15 & 6.78 & 55 & 0.7 & 20 & 1.09 & 0.147 & 1.7 & $<0.02$ & 0.42 & 1.71 \\
\hline $5 / 16 / 2008$ & 1.36 & 6.22 & 0.15 & 7.39 & 31 & 0.5 & 23 & 0.76 & 0.149 & 1.5 & $<0.02$ & 0.34 & 1.15 \\
\hline $5 / 16 / 2008$ & 1.57 & 5.81 & 0.17 & 8.73 & 64 & 1.3 & 34 & 0.77 & 0.307 & 1.2 & $<0.02$ & 0.43 & 1.63 \\
\hline 24/2008 & 1.03 & 4.05 & 0.24 & 7.28 & 45 & 11.4 & 27 & 2.49 & 0.216 & 0.9 & $<0.02$ & 0.2. & 2.78 \\
\hline $5 / 28 / 2008$ & 1.05 & 5.14 & 0.19 & 6.93 & 13 & 0.8 & 22 & 1.49 & 0.204 & 0.98 & $<0.02$ & 0.15 & 1.93 \\
\hline $7 / 15 / 2008$ & 24.5 & 133 & 0.46 & 13.8 & 9 & 0.7 & 245 & 0.22 & 0.213 & 3.8 & $<0.02$ & 2.2 & 2.83 \\
\hline 7/16/2008 & 33.0 & 129 & 0.40 & 18.9 & E6 & 0.8 & 294 & 0.08 & 0.184 & 4.6 & $<0.02$ & 4.9 & 0.82 \\
\hline 7/22/2008 & 22.2 & 54.9 & 0.32 & 11.0 & E6 & 18.8 & 138 & 1.44 & 0.150 & 8.0 & $<0.02$ & 35 & 3.57 \\
\hline $7 / 23 / 2000$ & 20.1 & 44.3 & 0.38 & 12.1 & 18 & 5.6 & 117 & 0.84 & 0.209 & 9.7 & $<0.02$ & 38 & 3.45 \\
\hline $8 / 712008$ & 23.6 & 49.3 & 0.59 & 10.6 & 12 & 0.0 & 215 & 0.09 & - & 3.1 & $<0.02$ & 1.4 & 0.93 \\
\hline 8/19/2000 & 26.9 & 11.4 & 0.26 & 16.6 & 14 & Е 0.3 & 123 & 0.63 & 0.185 & - & - & - & 1.43 \\
\hline 8/201/2008 & 1.60 & 3.92 & 0.24 & 8.19 & 62 & 0.5 & 32 & 0.97 & 0.217 & - & - & - & 1.47 \\
\hline 9/2/2008 & 2.79 & 8.71 & 0.22 & 12.5 & 35 & 0.6 & 41 & 1.21 & 0.352 & 4.8 & $<0.02$ & 4.3 & 3.35 \\
\hline 12008 & 0.91 & 2.12 & 0.19 & 6.06 & 57 & 0.7 & 28 & 0.35 & 0.146 & 3.8 & $<0.02$ & 2.3 & 0.67 \\
\hline 2008 & 0.67 & 1.65 & 0.18 & 6.61 & 60 & 0.5 & 31 & 0.11 & 0.143 & 2.8 & $<0.02$ & 2.0 & 0.54 \\
\hline 9/22/2008 & 1.20 & 2.07 & 0.28 & 6.01 & 518 & 16.7 & 69 & 0.14 & 0.293 & 7.4 & $<0.02$ & 1.8 & 0.95 \\
\hline
\end{tabular}

Table 5. Surface-water-quality data from samples collected at station 333150090530400, an unnamed tributary to Clear Creek near Napanee, MS.-Continued

[mg/L, milligram per liter; $\mathrm{SiO}_{2}$, silica dioxide; $\mu \mathrm{g} / \mathrm{L}$, microgram per liter; $\mathrm{UV}$, ultraviolet; $\mathrm{nm}$, nanometer; $\mathrm{NH}_{4}$, ammonium; -, no data; italic font indicates

\begin{tabular}{|c|c|c|c|c|c|c|c|c|c|c|c|c|c|}
\hline Date & $\begin{array}{l}\text { Speciitic UV } \\
\text { absorbance, } \\
\text { 254m, } \\
\text { water, } \\
\text { filtered }\end{array}$ & $\begin{array}{l}\text { Turbidity, } \\
\text { water, } \\
\text { unfitered, } \\
\text { in } \\
\text { Nephelo- } \\
\text { metric } \\
\text { Tutribitity } \\
\text { Units }\end{array}$ & $\begin{array}{l}\text { Dissolved } \\
\text { solids } \\
\text { dried } \\
\text { at 1880 } \\
\text { degres } \\
\text { Celsius, } \\
\text { water, } \\
\text { filtered, } \\
\text { mgl/l }\end{array}$ & $\begin{array}{l}\text { Dissolved } \\
\text { solids, } \\
\text { water, } \\
\text { filtered, } \\
\text { sum of } \\
\text { consitit } \\
\text { uentst, } \\
\text { mgl/ }\end{array}$ & $\begin{array}{c}\text { Dissolved } \\
\text { solids, } \\
\text { water. } \\
\text { tons } \\
\text { per day }\end{array}$ & $\begin{array}{l}\text { Dissolved } \\
\text { solids, } \\
\text { water, } \\
\text { filtered, tons } \\
\text { per acre-foot }\end{array}$ & $\begin{array}{l}\text { Ammonia, } \\
\text { water, } \\
\text { fitered, } \\
\text { mgl/L } \\
\text { as NH }\end{array}$ & $\begin{array}{c}\text { Nitrate, } \\
\text { water } \\
\text { fititered, } \\
\text { mg/L }\end{array}$ & $\begin{array}{c}\text { Nitrite, } \\
\text { water, } \\
\text { fitered, } \\
\text { my/h }\end{array}$ & $\begin{array}{c}\text { Suspended } \\
\text { sediment } \\
\text { concen- } \\
\text { tration, } \\
\text { mg/L }\end{array}$ & $\begin{array}{c}\text { Suspended } \\
\text { sediciment } \\
\text { discharge, } \\
\text { tons } \\
\text { per day }\end{array}$ & $\begin{array}{c}\text { Specific } \\
\text { conduc- } \\
\text { tance, } \\
\text { water, } \\
\text { unfitered, } \\
\text { laboratory, } \\
\text { microsie- } \\
\text { mens per } \\
\text { centimeter at } \\
\text { 25 degrees } \\
\text { Celsius }\end{array}$ & $\begin{array}{c}\text { Ratio of } \\
\text { partituculate } \\
\text { nitiogen to } \\
\text { particulate } \\
\text { organic } \\
\text { carbon, } \\
\text { number }\end{array}$ \\
\hline $4 / 1 / 2008$ & 2.7 & 780 & 71 & 51 & - & 0.10 & 0.039 & 4.39 & 0.104 & - & - & 83 & 0.151 \\
\hline $4 / 1 / 2008$ & - & $>1,000$ & 71 & 51 & 5.95 & 0.10 & 0.039 & 4.39 & 0.104 & 487 & 41 & 83 & 0.151 \\
\hline 1/42008 & 2.6 & $>1,000$ & 65 & 61 & 0.88 & 0.09 & 0.096 & 2.28 & 0.041 & 1,800 & 24 & 101 & 0.137 \\
\hline 4/4/2008 & 2.8 & 830 & 36 & 31 & 5.00 & 0.05 & 0.050 & 0.736 & 0.022 & 503 & 69 & 52 & 0.134 \\
\hline $4 / 5 / 2008$ & 2.3 & 510 & 36 & 31 & 4.88 & 0.05 & 0.044 & 0.556 & 0.018 & 343 & 46 & 50 & 0.138 \\
\hline $4 / 5 / 2008$ & 1.9 & 540 & 32 & 36 & 3.38 & 0.04 & 0.036 & 0.521 & 0.017 & 384 & 40 & 58 & 0.141 \\
\hline $4 / 5 / 2008$ & 2.5 & 460 & 43 & 39 & - & 0.06 & 0.085 & 0.571 & 0.023 & 347 & - & 63 & 0.169 \\
\hline $4 / 26 / 2008$ & 2.5 & $>1,000$ & 79 & 66 & - & 0.11 & 0.092 & 13.4 & 0.326 & 1,190 & - & 102 & 0.178 \\
\hline $4 / 26 / 2008$ & 2.3 & 86 & 93 & 75 & - & 0.13 & 0.088 & 15.5 & 0.440 & 714 & - & 113 & 0.175 \\
\hline $5 / 2 / 2008$ & 2.5 & $>1,000$ & 88 & 70 & 0.09 & 0.12 & 0.073 & 6.31 & 0.067 & 3,340 & 3.5 & 115 & 0.121 \\
\hline $5 / 3 / 2008$ & 3.0 & $>1,000$ & 40 & 37 & 2.47 & 0.05 & 0.085 & 2.17 & 0.084 & 578 & 36 & 57 & 0.164 \\
\hline $5 / 4 / 2008$ & 3.0 & 310 & 76 & 53 & E1.13 & 0.10 & 0.166 & 1.54 & 0.106 & 325 & E4.8 & 88 & 0.137 \\
\hline $5 / 4 / 2008$ & 3.1 & 310 & 89 & 61 & Е0.31 & 0.12 & 0.189 & 0.510 & 0.055 & 267 & Е0.94 & 101 & 0.149 \\
\hline $5 / 5 / 2008$ & 3.0 & 250 & 87 & 66 & 0.10 & 0.12 & 0.121 & 0.358 & 0.043 & 195 & 0.23 & 110 & 0.158 \\
\hline $5 / 14 / 2008$ & 2.3 & 350 & 161 & 146 & 0.30 & 0.22 & 0.063 & 21.8 & 0.132 & 309 & 0.58 & 245 & 0.181 \\
\hline $5 / 15 / 2008$ & 2.4 & $>1,000$ & 117 & 111 & 0.32 & 0.16 & 0.122 & 15.1 & 0.160 & 938 & 2.5 & 179 & 0.159 \\
\hline $5 / 15 / 2008$ & 2.4 & $>1,000$ & 56 & E43 & 6.23 & 0.08 & 0.065 & 5.08 & 0.051 & 580 & 64 & 66 & 0.142 \\
\hline $5 / 15 / 2008$ & 1.7 & 790 & 53 & E44 & 5.55 & 0.07 & 0.072 & 4.33 & 0.052 & 418 & 44 & 68 & 0.167 \\
\hline $5 / 15 / 2000$ & 2.6 & 610 & 43 & 40 & 3.37 & 0.06 & 0.079 & 3.10 & 0.057 & 351 & 27 & 64 & 0.171 \\
\hline $5 / 16 / 2008$ & 2.6 & 600 & 61 & 45 & - & 0.08 & 0.069 & 2.72 & 0.050 & 337 & - & 69 & 0.151 \\
\hline $5 / 16 / 2008$ & 3.1 & 260 & 74 & 57 & - & 0.10 & 0.269 & 1.24 & 0.074 & 199 & - & 92 & 0.135 \\
\hline $5 / 242008$ & 3.1 & $>1,000$ & 47 & 46 & - & 0.06 & 0.179 & 2.83 & 0.119 & 5,060 & - & 73 & 0.143 \\
\hline $5 / 28 / 2008$ & 3.0 & $>1,000$ & 54 & 41 & - & 0.07 & 0.077 & 1.69 & 0.049 & 784 & - & 63 & 0.132 \\
\hline 7/15/2008 & 2.3 & 16 & 504 & 488 & 1.22 & 0.69 & 0.267 & 7.03 & 0.231 & 193 & 0.47 & 783 & 0.173 \\
\hline 7/16/2008 & 2.4 & 5.0 & 554 & E543 & 8.38 & 0.75 & 0.081 & 1.32 & 0.055 & 106 & 1.6 & 857 & 0.147 \\
\hline 7/222/2008 & 2.1 & $>1,000$ & 301 & E271 & 1.54 & 0.41 & Е0.019 & 8.07 & 0.145 & 735 & 3.8 & 464 & 0.176 \\
\hline 7/23/2008 & 2.2 & 260 & 267 & 239 & 13.0 & 0.36 & - & 10.1 & 0.322 & 243 & 12 & 410 & 0.222 \\
\hline $8 / 72008$ & - & 6.0 & 356 & $E 329$ & - & 0.48 & 0.167 & $E 0.065$ & 0.024 & 297 & - & 569 & 0.101 \\
\hline 8/19/2008 & 3.1 & 540 & 208 & E201 & 5.17 & 0.28 & Е0.022 & 2.03 & 0.027 & 386 & 9.6 & 349 & 0.159 \\
\hline $8 / 20 / 2008$ & 3.2 & 460 & 63 & 53 & - & 0.09 & 0.027 & 1.54 & 0.021 & - & - & $E 82$ & 0.190 \\
\hline 9/2/2008 & 3.5 & 320 & 99 & 83 & - & 0.14 & 0.097 & 7.83 & 0.060 & 403 & - & 127 & 0.187 \\
\hline 9/3/2008 & 3.0 & 87 & 43 & 40 & - & 0.06 & 0.057 & 0.387 & 0.021 & 151 & - & $E 62$ & 0.130 \\
\hline 9/4/2008 & 3.0 & 20 & 48 & $E 42$ & - & 0.07 & 0.129 & $E 0.084$ & 0.010 & 115 & - & E66 & 0.128 \\
\hline 9/22/20008 & 3.1 & 10 & 114 & 81 & - & 0.15 & 0.057 & $<0.163$ & 0.010 & 99 & - & 143 & 0.114 \\
\hline
\end{tabular}


Table 6. Land managers' report of physical and chemical treatments of fields during crop production, Tommie Bayou Basin, Mississippi, 2007.

[NA, no treatment; pt., pint; MRVA, Mississippi River valley aquifer; oz., ounce; \#, pound; qt., quart; GPM, gallon per minute; hrs., hours; see fig. 5 for locations]

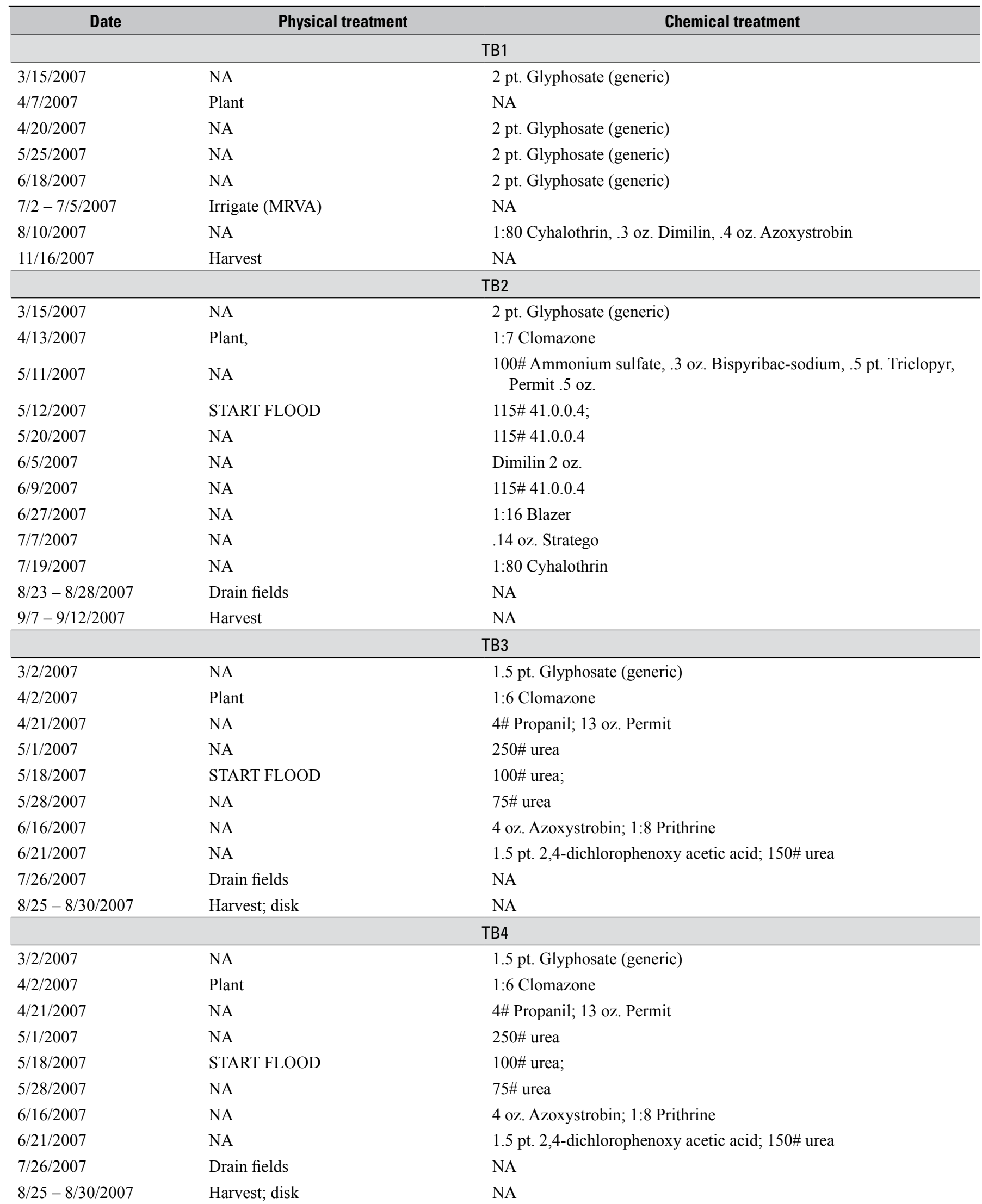


Table 6. Land managers' report of physical and chemical treatments of fields during crop production, Tommie Bayou Basin, Mississippi, 2007.-Continued

[NA, no treatment; pt., pint; MRVA, Mississippi River valley aquifer; oz., ounce; \#, pound; qt., quart; GPM, gallon per minute; hrs., hours; see fig. 5 for locations]

\begin{tabular}{|c|c|c|}
\hline Date & Physical treatment & Chemical treatment \\
\hline \multicolumn{3}{|r|}{ TB5 } \\
\hline $3 / 2 / 2007$ & NA & 1.5 pt. Glyphosate (generic) \\
\hline $4 / 21 / 2007$ & NA & 4\# Propanil; 13o z. Permit \\
\hline $5 / 1 / 2007$ & NA & $250 \#$ urea \\
\hline $5 / 28 / 2007$ & NA & 75\# urea \\
\hline $6 / 16 / 2007$ & NA & 4 oz. Azoxystrobin; 1:8 Prithrine \\
\hline $6 / 21 / 2007$ & NA & 1.5 pt. 2,4-dichlorophenoxy acetic acid; 150\# urea \\
\hline $7 / 26 / 2007$ & Drain fields & NA \\
\hline $8 / 25-8 / 30 / 2007$ & Harvest; disk & NA \\
\hline $4 / 21 / 2007$ & NA & 4\# Propanil; 13 oz. Permit \\
\hline $5 / 1 / 2007$ & NA & $250 \#$ urea \\
\hline $5 / 18 / 2007$ & START FLOOD & 100\# urea; \\
\hline $5 / 28 / 2007$ & NA & 75\# urea \\
\hline $6 / 16 / 2007$ & NA & 4 oz. Azoxystrobin; 1:8 Prithrine \\
\hline $6 / 21 / 2007$ & NA & 1.5 pt. 2,4-dichlorophenoxy acetic acid; 150\# urea \\
\hline $7 / 26 / 2007$ & Drain fields & NA \\
\hline $8 / 25-8 / 30 / 2007$ & Harvest; disk & NA \\
\hline $5 / 18 / 2007$ & START FLOOD & 100\# urea \\
\hline $6 / 21 / 2007$ & NA & $150 \#$ urea; 1.5 pt. 2,4-dichlorophenoxy acetic acid \\
\hline $7 / 26 / 2007$ & Drain & NA \\
\hline $8 / 25-8 / 30 / 2007$ & Harvest, disk & NA \\
\hline \multicolumn{3}{|r|}{ TB8 } \\
\hline $3 / 2 / 2007$ & NA & 1.5 pt. Glyphosate (generic) \\
\hline $4 / 3 / 2007$ & Plant & 1:6 Clomazone \\
\hline $4 / 7-4 / 9 / 2007$ & Flushed (watered) & NA \\
\hline $5 / 1 / 2007$ & NA & 100\# Ammonium sulfate \\
\hline $5 / 1 / 2007$ & NA & 250\# urea \\
\hline $5 / 18 / 2007$ & START FLOOD & $100 \#$ urea \\
\hline $6 / 21 / 2007$ & NA & $150 \#$ urea; 1.5 pt. 2,4-dichlorophenoxy acetic acid \\
\hline $7 / 26 / 2007$ & Drain & NA \\
\hline $8 / 25-8 / 30 / 2007$ & Harvest, disk & NA \\
\hline
\end{tabular}


Table 6. Land managers' report of physical and chemical treatments of fields during crop production, Tommie Bayou Basin, Mississippi, 2007.-Continued

[NA, no treatment; pt., pint; MRVA, Mississippi River valley aquifer; oz., ounce; \#, pound; qt., quart; GPM, gallon per minute; hrs., hours; see fig. 5 for locations]

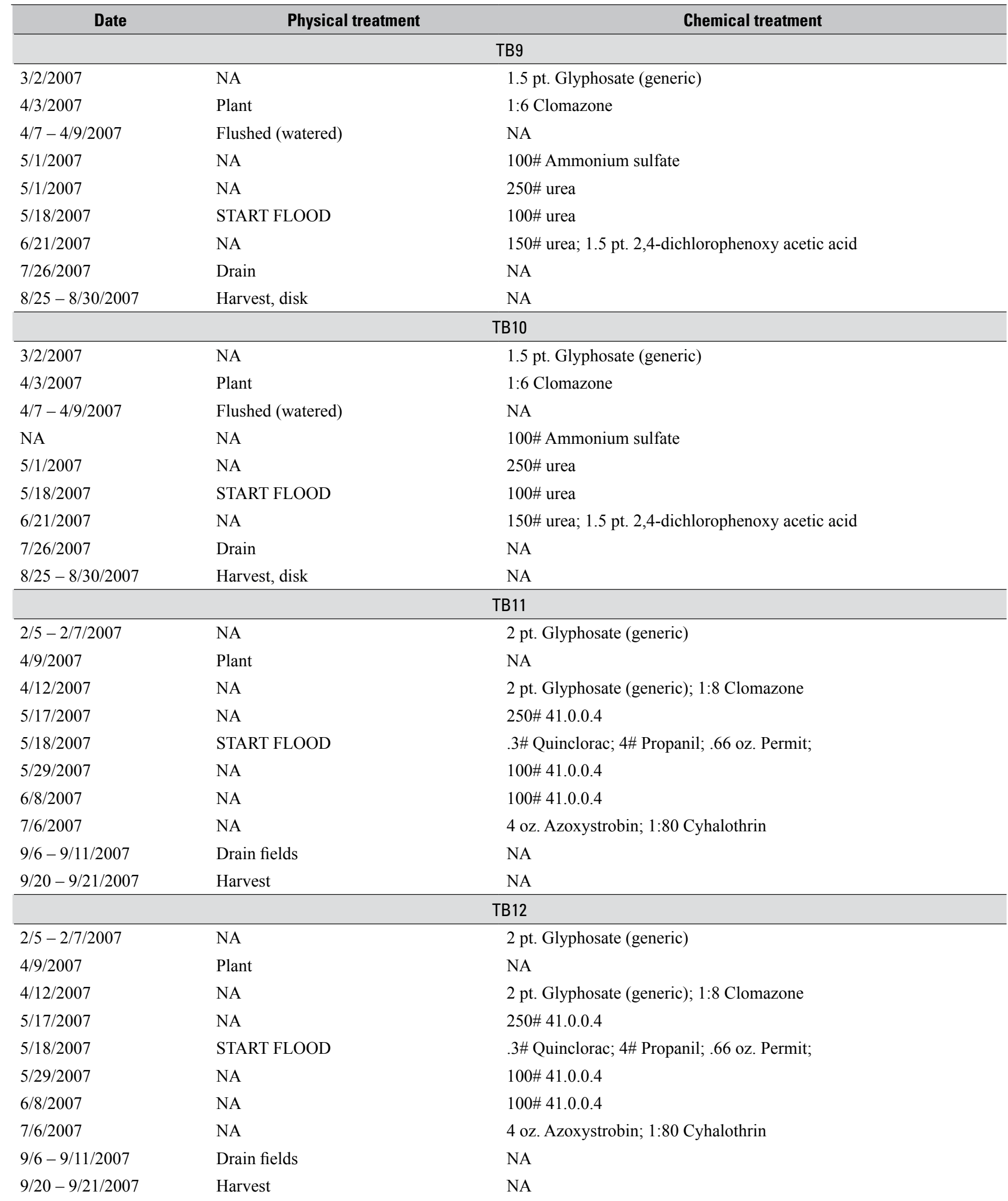


Table 6. Land managers' report of physical and chemical treatments of fields during crop production, Tommie Bayou Basin, Mississippi, 2007.-Continued

[NA, no treatment; pt., pint; MRVA, Mississippi River valley aquifer; oz., ounce; \#, pound; qt., quart; GPM, gallon per minute; hrs., hours; see fig. 5 for locations]

\begin{tabular}{|c|c|c|}
\hline Date & Physical treatment & Chemical treatment \\
\hline \multicolumn{3}{|c|}{ TB13 } \\
\hline $2 / 5-2 / 7 / 2007$ & NA & 2 pt. Glyphosate (generic) \\
\hline $5 / 17 / 2007$ & NA & $250 \# 41.0 .0 .4$ \\
\hline $5 / 19 / 2007$ & START FLOOD & .3\# Quinclorac; 4\# Propanil; .66 oz. Permit; \\
\hline $7 / 6 / 2007$ & NA & 4 oz. Azoxystrobin \\
\hline $8 / 8 / 2007$ & NA & 1:8 Cyhalothrin \\
\hline $8 / 20-8 / 23 / 2007$ & Drain fields & NA \\
\hline $9 / 3 / 2007$ & Harvest & NA \\
\hline $5 / 17 / 2007$ & NA & $250 \# 41.0 .0 .4$ \\
\hline $5 / 19 / 2007$ & START FLOOD & .3\# Quinclorac; 4\# Propanil; .66 oz. Permit \\
\hline $5 / 29 / 2007$ & NA & $100 \# 41.0 .0 .4$ \\
\hline $6 / 8 / 2007$ & NA & $100 \# 41.0 .0 .4$ \\
\hline $7 / 6 / 2007$ & NA & 4 oz. Azoxystrobin \\
\hline $8 / 8 / 2007$ & NA & 1:8 Cyhalothrin \\
\hline $8 / 20-8 / 23 / 2007$ & Drain fields & NA \\
\hline $9 / 3 / 2007$ & Harvest & NA \\
\hline \multicolumn{3}{|c|}{ TB15 } \\
\hline $7 / 6 / 2007$ & NA & 4 oz. Azoxystrobin \\
\hline $8 / 8 / 2007$ & NA & 1:8 Cyhalothrin \\
\hline $8 / 20-8 / 23 / 2007$ & Drain fields & NA \\
\hline $9 / 3 / 2007$ & Harvest & NA \\
\hline \multicolumn{3}{|c|}{ TB16 } \\
\hline $2 / 5-2 / 7 / 2007$ & NA & 2 pt. Glyphosate (generic) \\
\hline $4 / 6-4 / 8 / 2007$ & Plant & 2 pt. Glyphosate (generic); 1:7 Clomazone \\
\hline $5 / 17 / 2007$ & NA & $250 \# 41.0 .0 .4$ \\
\hline $5 / 19 / 2007$ & START FLOOD & .3\# Quinclorac; 4\# Propanil; .66 oz. Permit \\
\hline $5 / 29 / 2007$ & NA & $100 \# 41.0 .0 .4$ \\
\hline $6 / 8 / 2007$ & NA & $100 \# 41.0 .0 .4$ \\
\hline $7 / 6 / 2007$ & NA & 4 oz. Azoxystrobin \\
\hline $8 / 8 / 2007$ & NA & 1:8 Cyhalothrin \\
\hline $8 / 20-8 / 23 / 2007$ & Drain fields & NA \\
\hline $9 / 3 / 2007$ & Harvest & NA \\
\hline
\end{tabular}


Table 6. Land managers' report of physical and chemical treatments of fields during crop production, Tommie Bayou Basin, Mississippi, 2007.-Continued

[NA, no treatment; pt., pint; MRVA, Mississippi River valley aquifer; oz., ounce; \#, pound; qt., quart; GPM, gallon per minute; hrs., hours; see fig. 5 for locations]

\begin{tabular}{|c|c|c|}
\hline Date & Physical treatment & Chemical treatment \\
\hline \multicolumn{3}{|c|}{ TB17 } \\
\hline $2 / 5 /-2 / 7 / 2007$ & NA & 2 pt. Glyphosate (generic) \\
\hline $5 / 1 / 2007$ & NA & 2 pt. Glyphosate (generic) \\
\hline $5 / 20 / 2007$ & NA & 2 pt. Glyphosate (generic) \\
\hline $10 / 20 / 2007$ & Disk & NA \\
\hline \multicolumn{3}{|c|}{ TB18 } \\
\hline $5 / 21 / 2007$ & Plant & NA \\
\hline $5 / 8 / 2007$ & NA & 3 pt. Glyphosate (generic) \\
\hline $7 / 28 / 2007$ & NA & 3 pt. Glyphosate (generic) \\
\hline $2 / 22 / 2007$ & NA & 2 pt. Glyphosate (generic) \\
\hline $4 / 27 / 2007$ & Plant & NA \\
\hline $6 / 5 / 2007$ & NA & 3 pt. Glyphosate (generic) \\
\hline $7 / 28 / 2007$ & NA & 3 pt. Glyphosate (generic) \\
\hline $7 / 29 / 2007$ & NA & 1:80 Cyhalothrin, 2 oz. Dimilin, 4 oz. Azoxystrobin \\
\hline $8 / 13-8 / 15 / 2007$ & Irrigated (MRVA) & NA \\
\hline $8 / 15 / 2007$ & NA & 1:80 Cyhalothrin \\
\hline $10 / 31-11 / 1 / 2007$ & Harvest & NA \\
\hline $8 / 13-8 / 15 / 2007$ & Irrigated (MRVA) & NA \\
\hline $8 / 15 / 2007$ & NA & 1:80 Cyhalothrin \\
\hline $10 / 31-11 / 1 / 2007$ & Harvest & NA \\
\hline \multicolumn{3}{|c|}{ TB21 } \\
\hline $4 / 21 / 2007$ & Plant & NA \\
\hline $5 / 2 / 2007$ & NA & 3 pt. Glyphosate (generic) \\
\hline $8 / 20-8 / 22 / 2007$ & Irrigate & NA \\
\hline $9 / 1-9 / 2 / 2007$ & Harvest & NA \\
\hline \multicolumn{3}{|c|}{ TB22 } \\
\hline $2 / 28 / 2007$ & NA & 1.5 pt. Glyphosate (generic); 2 pt. 2,4-dichlorophenoxy acetic acid \\
\hline $5 / 21 / 2007$ & Plant & NA \\
\hline $5 / 30 / 2007$ & NA & 3 pt. Glyphosate (generic) \\
\hline $6 / 21-6 / 22 / 2007$ & NA & 2 pt. Glyphosate (generic) \\
\hline $8 / 24 / 2007$ & NA & 3 oz. Intrepid; .6 oz. Acceplate \\
\hline $9 / 25-9 / 26 / 2007$ & Harvest & NA \\
\hline
\end{tabular}


Table 6. Land managers' report of physical and chemical treatments of fields during crop production, Tommie Bayou Basin, Mississippi, 2007.-Continued

[NA, no treatment; pt., pint; MRVA, Mississippi River valley aquifer; oz., ounce; \#, pound; qt., quart; GPM, gallon per minute; hrs., hours; see fig. 5 for locations]

\begin{tabular}{|c|c|c|}
\hline Date & Physical treatment & Chemical treatment \\
\hline \multicolumn{3}{|c|}{ TB23 } \\
\hline $4 / 20 / 2007$ & Plant & 1 qt. Glyphosate (generic), 1:7 Clomazone \\
\hline $5 / 16 / 2007$ & START FLOOD & .4\# Quinclorac, 3\# Propanil; 250\# 41.0.0.4 \\
\hline $6 / 16 / 2007$ & NA & $100 \# 41.0 .0 .4$ \\
\hline $8 / 16 / 2007$ & Drain rice & NA \\
\hline $8 / 25-8 / 28 / 2007$ & Harvest & NA \\
\hline \multicolumn{3}{|c|}{ TB24 } \\
\hline $2 / 28 / 2007$ & NA & 1.5 pt. Glyphosate (generic); 2 pt. 2,4-dichlorophenoxy acetic acid \\
\hline $5 / 21 / 2007$ & Plant & NA \\
\hline $9 / 25-9 / 26 / 2007$ & Harvest & NA \\
\hline \multicolumn{3}{|c|}{ TB25 } \\
\hline $4 / 20 / 2007$ & Plant & 1 qt. Glyphosate (generic), 1:7 Clomazone \\
\hline $5 / 2 / 2007$ & NA & 100\# Ammonium sulfate \\
\hline $5 / 16 / 2007$ & START FLOOD & .4\# Quinclorac, 3\# Propanil; 250\# 41.0.0.4 \\
\hline $6 / 16 / 2007$ & NA & $100 \# 41.0 .0 .4$ \\
\hline $7 / 5 / 2007$ & NA & 1 qt. 2,4-dichlorophenoxy acetic acid on levee; $100 \#$ urea on fields \\
\hline $8 / 16 / 2007$ & Drain rice & NA \\
\hline $8 / 24 / 2007$ & NA & 3 oz. Intrepid; .6 oz. Acceplate \\
\hline $9 / 25-9 / 26 / 2007$ & Harvest & NA \\
\hline \multicolumn{3}{|c|}{ TB27 } \\
\hline
\end{tabular}

4/20/2007 Plant

$5 / 2 / 2007 \quad$ NA

$5 / 16 / 2007$

START FLOOD

$6 / 16 / 2007$

NA

$7 / 5 / 2007$

NA

$8 / 16 / 2007$

$8 / 25-8 / 28 / 2007$

Drain rice

Harvest
1 qt. Glyphosate (generic), 1:7 Clomazone

100\# Ammonium sulfate

.4\# Quinclorac, 3\# Propanil; 250\# 41.0.0.4

100\# 41.0.0.4

1 qt. 2,4-dichlorophenoxy acetic acid on levee; 100\# urea on fields

NA

NA 
Table 6. Land managers' report of physical and chemical treatments of fields during crop production, Tommie Bayou Basin, Mississippi, 2007.-Continued

[NA, no treatment; pt., pint; MRVA, Mississippi River valley aquifer; oz., ounce; \#, pound; qt., quart; GPM, gallon per minute; hrs., hours; see fig. 5 for locations]

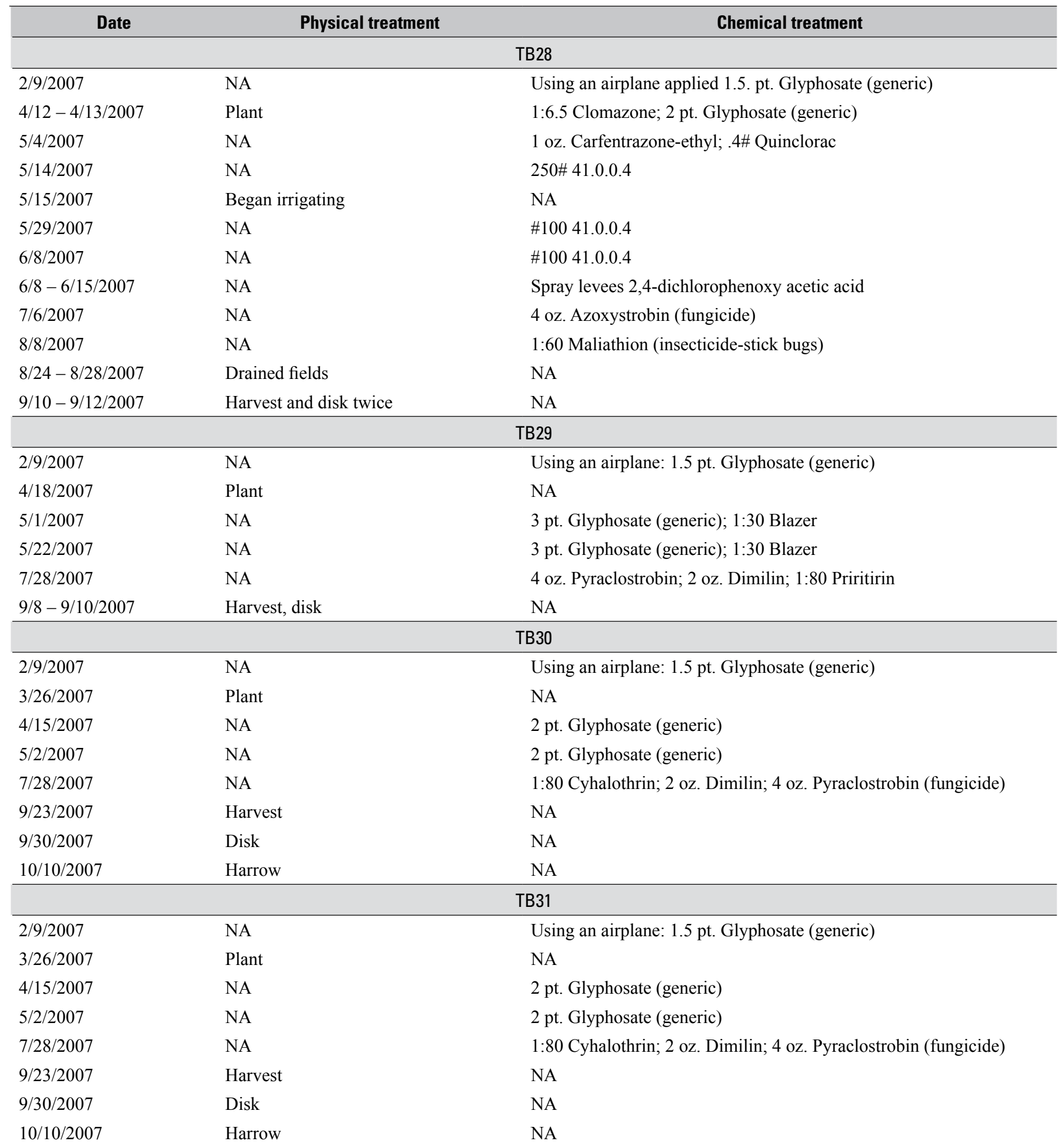


Table 6. Land managers' report of physical and chemical treatments of fields during crop production, Tommie Bayou Basin, Mississippi, 2007.-Continued

[NA, no treatment; pt., pint; MRVA, Mississippi River valley aquifer; oz., ounce; \#, pound; qt., quart; GPM, gallon per minute; hrs., hours; see fig. 5 for locations]

\begin{tabular}{|c|c|c|}
\hline Date & Physical treatment & Chemical treatment \\
\hline \multicolumn{3}{|c|}{ TB32 } \\
\hline $2 / 9 / 2007$ & NA & Using an airplane: 1.5 pt. Glyphosate (generic) \\
\hline $5 / 4 / 2007$ & NA & 4\# Propanil; .66 pt. Triclopyr; .3\# Quinclorac \\
\hline $5 / 14 / 2007$ & NA & $250 \# 41.0 .0 .4$ \\
\hline $5 / 29 / 2007$ & NA & \#100 41.0.0.4 \\
\hline $6 / 8 / 2007$ & NA & $\# 100$ 41.0.0.4 \\
\hline $6 / 8-6 / 15 / 2007$ & NA & Spray levees 2,4-dichlorophenoxy acetic acid \\
\hline $7 / 6 / 2007$ & NA & $4 \mathrm{oz}$. Azoxystrobin (fungicide) \\
\hline $8 / 8 / 2007$ & NA & 1:60 Maliathion (insecticide-stick bugs) \\
\hline \multicolumn{3}{|c|}{ TB33 } \\
\hline $4 / 18 / 2007$ & Plant & NA \\
\hline $5 / 10 / 2007$ & NA & 3 pt. Glyphosate (generic) \\
\hline $6 / 20 / 2007$ & NA & 3 pt. Glyphosate (generic) \\
\hline $7 / 28 / 2007$ & NA & 1:75 Cyhalothrin; irrigated (MRVA) \\
\hline $8 / 10 / 2007$ & NA & 1:75 Cyhalothrin \\
\hline $10 / 15 / 2007$ & Harvest & NA \\
\hline $10 / 16-10 / 18 / 2007$ & Disk, land plane & NA \\
\hline \multicolumn{3}{|c|}{ TB34 } \\
\hline $10 / 16-10 / 18 / 2007$ & Disk, land plane & NA \\
\hline \multicolumn{3}{|c|}{ TB35 } \\
\hline $4 / 20 / 2007$ & Plant & .5 pt. Triclopyr, .5 oz. Permit \\
\hline $5 / 8 / 2007$ & NA & 100\# Ammonium sulfate \\
\hline $5 / 16 / 2007$ & START FLOOD & $115 \# 41.0 .0 .4$ \\
\hline $6 / 5 / 2007$ & NA & $115 \# 41.0 .0 .4$ \\
\hline $6 / 15 / 2007$ & NA & $100 \# 41.0 .0 .4$ \\
\hline $6 / 18 / 2007$ & NA & .4 oz. Azoxystrobin, 1:71 Cyhalothrin \\
\hline $8 / 24-8 / 28 / 2007$ & Drained fields & NA \\
\hline $9 / 8 / 2007$ & Harvest & NA \\
\hline $9 / 11-9 / 12 / 2007$ & Disk and smooth & NA \\
\hline
\end{tabular}


Table 6. Land managers' report of physical and chemical treatments of fields during crop production, Tommie Bayou Basin, Mississippi, 2007.-Continued

[NA, no treatment; pt., pint; MRVA, Mississippi River valley aquifer; oz., ounce; \#, pound; qt., quart; GPM, gallon per minute; hrs., hours; see fig. 5 for locations]

\begin{tabular}{|c|c|c|}
\hline Date & Physical treatment & Chemical treatment \\
\hline \multicolumn{3}{|c|}{ TB36 } \\
\hline $2 / 8 / 2007$ & NA & 100\# Ammonium sulfate (fertilizer) \\
\hline $2 / 22 / 2007$ & NA & $.6 \mathrm{oz}$. Thifensulfuron methyl \\
\hline $5 / 29 / 2007$ & Harvest & NA \\
\hline \multicolumn{3}{|c|}{ TB37 } \\
\hline $3 / 5 / 2007$ & NA & 1 pt. 2,4-dichlorophenoxy acetic acid; 1 qt. Glyphosate (generic) \\
\hline $4 / 19 / 2007$ & Planted & NA \\
\hline $4 / 15 / 2007$ & NA & 2 pt. Glyphosate (generic) \\
\hline $5 / 8 / 2007$ & NA & 2 pt. Glyphosate (generic) \\
\hline $4 / 19-4 / 23 / 2007$ & Plant & NA \\
\hline $4 / 30 / 2007$ & NA & 2 pt. Glyphosate (generic) \\
\hline $7 / 1 / 2007$ & NA & 2 pt. Glyphosate (generic) \\
\hline $7 / 21 / 2007$ & NA & 4 oz. Pyraclostrobin; 2 oz. Dimilin; 1:80 Cyhalothrin \\
\hline $8 / 12-8 / 13 / 2007$ & Harvest & NA \\
\hline \multicolumn{3}{|c|}{ TB39 } \\
\hline $1 / 10 / 2007$ & NA & Using an airplane applied: 1.5 pt. Glyphosate (generic) \\
\hline $4 / 19-4 / 23 / 2007$ & Plant & NA \\
\hline $3 / 12 / 2007$ & Harrow & NA \\
\hline $4 / 14 / 2007$ & Field cultivate & NA \\
\hline $4 / 22 / 2007$ & Harrow & NA \\
\hline $5 / 10-5 / 15 / 2007$ & Plant & NA \\
\hline $5 / 30 / 2007$ & NA & 2 pt. Glyphosate (generic) \\
\hline $6 / 8 / 2007$ & NA & 2 pt. Glyphosate (generic) \\
\hline $6 / 11 / 2007$ & NA & $.4 \mathrm{oz}$. Azoxystrobin; EDTA, copper complex insecticide 1:70 \\
\hline $7 / 2-7 / 5 / 2007$ & Irrigated (MRVA) & NA \\
\hline $10 / 15-10 / 17 / 2007$ & Harvest & NA \\
\hline
\end{tabular}


Table 6. Land managers' report of physical and chemical treatments of fields during crop production, Tommie Bayou Basin, Mississippi, 2007.-Continued

[NA, no treatment; pt., pint; MRVA, Mississippi River valley aquifer; oz., ounce; \#, pound; qt., quart; GPM, gallon per minute; hrs., hours; see fig. 5 for locations]

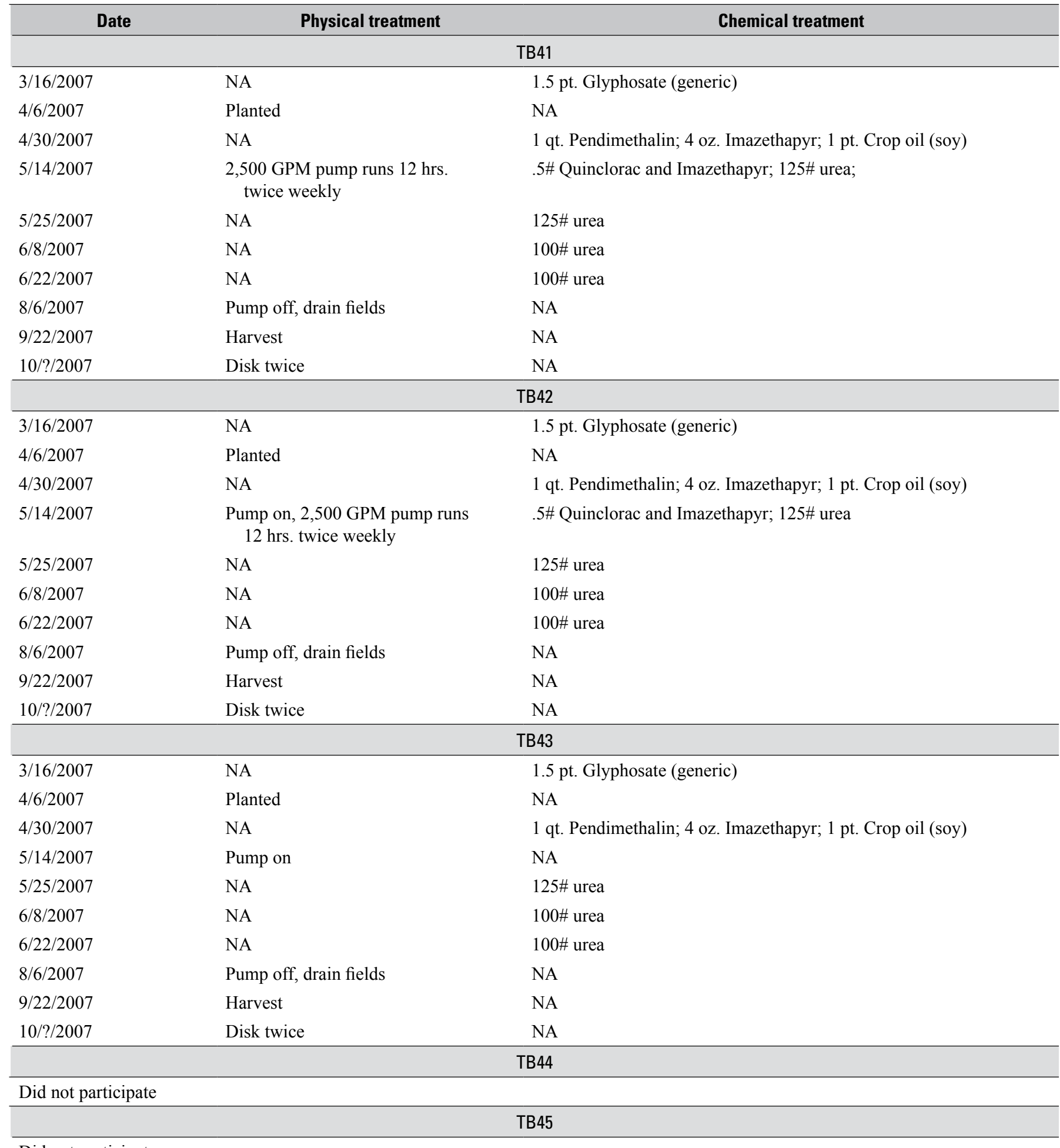

Did not participate 
Table 7. Land managers' report of physical and chemical treatments of fields during crop production, Tommie Bayou Basin, Mississippi, 2008.

[NA, no treatment; oz, ounce; \#, pound; qt., quart; MRVA, Mississippi River valley aquifer; pt., pint; GPA, gallons per acre; \%, percent; see fig. 6 for locations]

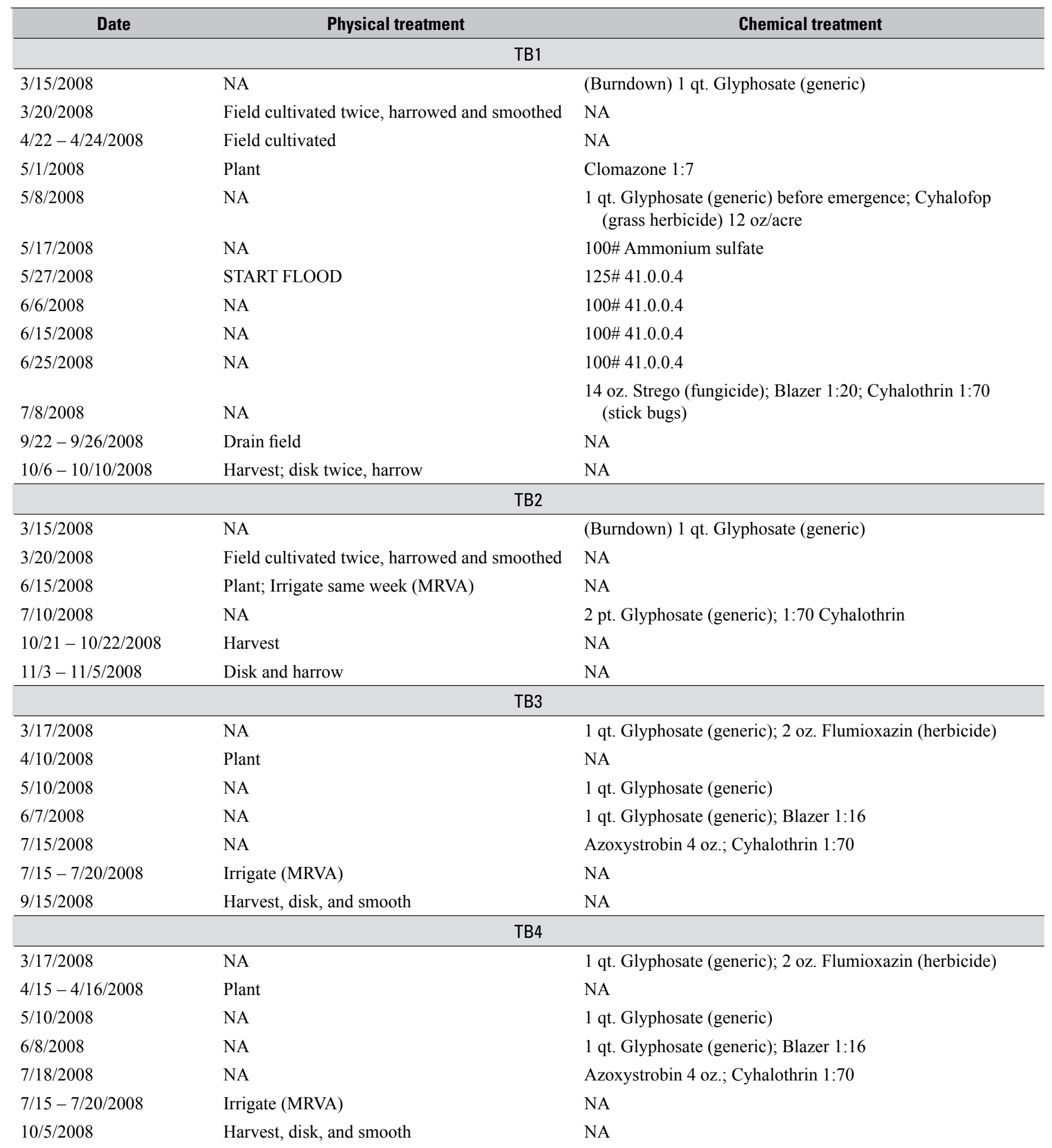


Table 7. Land managers' report of physical and chemical treatments of fields during crop production, Tommie Bayou Basin, Mississippi, 2008. - Continued

[NA, no treatment; oz, ounce; \#, pound; qt., quart; MRVA, Mississippi River valley aquifer; pt., pint; GPA, gallons per acre; \%, percent; see fig. 6 for locations]

\begin{tabular}{|c|c|c|}
\hline Date & Physical treatment & Chemical treatment \\
\hline \multicolumn{3}{|c|}{ TB5 } \\
\hline $3 / 17 / 2008$ & NA & 1 qt. Glyphosate (generic); 2 oz. Flumioxazin (herbicide) \\
\hline $5 / 10 / 2008$ & NA & 1 qt. Glyphosate (generic) \\
\hline $6 / 8 / 2008$ & NA & 1 qt. Glyphosate (generic); Blazer 1:16 \\
\hline $7 / 15-7 / 20 / 2008$ & Irrigate (MRVA) & NA \\
\hline $10 / 5 / 2008$ & Harvest, disk, and smooth & NA \\
\hline \multicolumn{3}{|c|}{ TB6 } \\
\hline $3 / 17 / 2008$ & NA & 1 qt. Glyphosate (generic); 2 oz. Flumioxazin (herbicide) \\
\hline $4 / 15-4 / 16 / 2008$ & Plant & NA \\
\hline $7 / 18 / 2008$ & NA & Azoxystrobin 4 oz.; Cyhalothrin 1:70 \\
\hline $7 / 15-7 / 20 / 2008$ & Irrigate (MRVA) & NA \\
\hline $10 / 5 / 2008$ & Harvest, disk, and smooth & NA \\
\hline \multicolumn{3}{|c|}{ TB7 } \\
\hline $3 / 17 / 2008$ & NA & 1 qt. Glyphosate (generic); 2 oz. Flumioxazin (herbicide) \\
\hline $4 / 15-4 / 16 / 2008$ & Plant & NA \\
\hline $5 / 10 / 2008$ & NA & 1 qt. Glyphosate (generic) \\
\hline $6 / 8 / 2008$ & NA & 1 qt. Glyphosate (generic); Blazer 1:16 \\
\hline $7 / 18 / 2008$ & NA & Azoxystrobin 4 oz.; Cyhalothrin 1:70 \\
\hline $6 / 8 / 2008$ & NA & 1 qt. Glyphosate (generic); Blazer 1:16 \\
\hline $7 / 18 / 2008$ & NA & Azoxystrobin 4 oz.; Cyhalothrin 1:70 \\
\hline $7 / 15-7 / 20 / 2008$ & Irrigate (MRVA) & NA \\
\hline $10 / 5 / 2008$ & Harvest, disk, and smooth & NA \\
\hline \multicolumn{3}{|c|}{ TB9 } \\
\hline $3 / 17 / 2008$ & NA & 1 qt. Glyphosate (generic); 2 oz. Flumioxazin (herbicide) \\
\hline $4 / 15-4 / 16 / 2008$ & Plant & NA \\
\hline $5 / 10 / 2008$ & NA & 1 qt. Glyphosate (generic) \\
\hline $6 / 8 / 2008$ & NA & 1 qt. Glyphosate (generic); Blazer 1:16 \\
\hline $7 / 18 / 2008$ & NA & Azoxystrobin 4 oz.; Cyhalothrin 1:70 \\
\hline $7 / 15-7 / 20 / 2008$ & Irrigate (MRVA) & NA \\
\hline $10 / 5 / 2008$ & Harvest, disk, and smooth & NA \\
\hline
\end{tabular}


Table 7. Land managers' report of physical and chemical treatments of fields during crop production, Tommie Bayou Basin, Mississippi, 2008.-Continued

[NA, no treatment; oz, ounce; \#, pound; qt., quart; MRVA, Mississippi River valley aquifer; pt., pint; GPA, gallons per acre; \%, percent; see fig. 6 for locations]

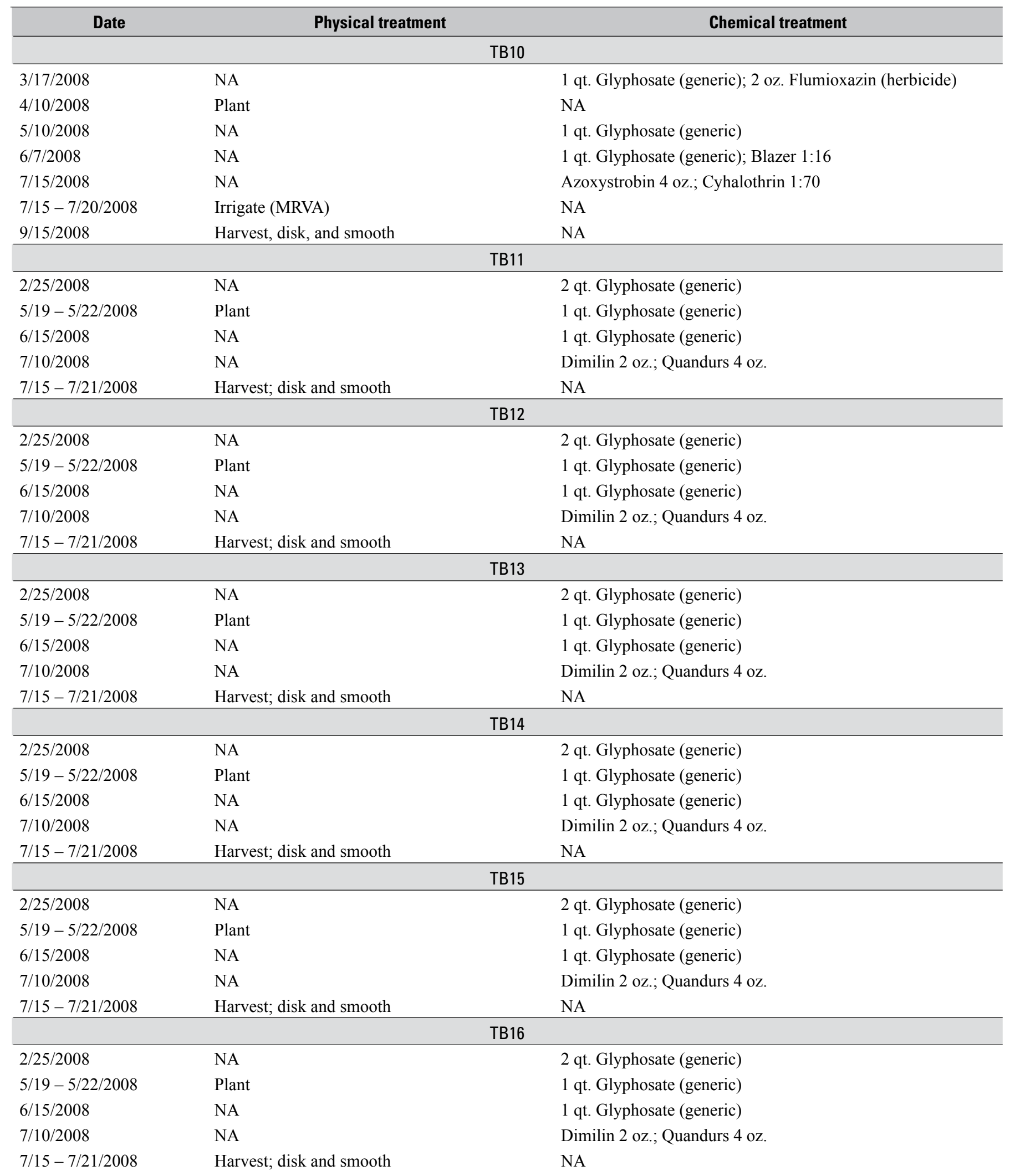


Table 7. Land managers' report of physical and chemical treatments of fields during crop production, Tommie Bayou Basin, Mississippi, 2008. - Continued

[NA, no treatment; oz, ounce; \#, pound; qt., quart; MRVA, Mississippi River valley aquifer; pt., pint; GPA, gallons per acre; \%, percent; see fig. 6 for locations]




Table 7. Land managers' report of physical and chemical treatments of fields during crop production, Tommie Bayou Basin, Mississippi, 2008.-Continued

[NA, no treatment; oz, ounce; \#, pound; qt., quart; MRVA, Mississippi River valley aquifer; pt., pint; GPA, gallons per acre; \%, percent; see fig. 6 for locations]




Table 7. Land managers' report of physical and chemical treatments of fields during crop production, Tommie Bayou Basin, Mississippi, 2008. - Continued

[NA, no treatment; oz, ounce; \#, pound; qt., quart; MRVA, Mississippi River valley aquifer; pt., pint; GPA, gallons per acre; \%, percent; see fig. 6 for locations]




Table 7. Land managers' report of physical and chemical treatments of fields during crop production, Tommie Bayou Basin, Mississippi, 2008.-Continued

[NA, no treatment; oz, ounce; \#, pound; qt., quart; MRVA, Mississippi River valley aquifer; pt., pint; GPA, gallons per acre; \%, percent; see fig. 6 for locations]

\begin{tabular}{|c|c|c|}
\hline Date & Physical treatment & Chemical treatment \\
\hline $3 / 10-3 / 12 / 2008$ & NA & (Burndown) 1.5 pt. Glyphosate (generic) \\
\hline $5 / 19 / 2008$ & NA & Propanil 4\#; Carfentrazone-ethyl $1 \mathrm{oz.}$ \\
\hline $5 / 20 / 2008$ & START FLOOD & $150 \# 41.0 .0 .4$ \\
\hline 6/9/2008 & NA & $100 \# 4.10 .0 .4$ \\
\hline $6 / 8 / 2008$ & NA & $\begin{array}{l}\text { Bispyribac-sodium } 0.5 \text { oz.; Quinclorac } 0.5 \# \text {; Zeta-cyperme- } \\
\text { thrin 1:40; Dynapact } 1 \%\end{array}$ \\
\hline $9 / 15-9 / 19 / 2008$ & Drained Fields & NA \\
\hline $9 / 29-10 / 4 / 2008$ & Harvest & NA \\
\hline $3 / 10-3 / 12 / 2008$ & NA & (Burndown) 1.5 pt. Glyphosate (generic) \\
\hline $4 / 13 / 2008$ & Plant & Clomazone 1:7 \\
\hline $5 / 19 / 2008$ & NA & Propanil 4\#; Carfentrazone-ethyl $1 \mathrm{oz.}$ \\
\hline $5 / 20 / 2008$ & START FLOOD & $150 \# 41.0 .0 .4$ \\
\hline $5 / 31 / 2008$ & NA & $100 \# 4.10 .0 .4$ \\
\hline $6 / 9 / 2008$ & NA & $100 \# 4.10 .0 .4$ \\
\hline $6 / 8 / 2008$ & NA & $\begin{array}{l}\text { Bispyribac-sodium } 0.5 \text { oz.; Quinclorac } 0.5 \# \text {; Zeta-cyperme- } \\
\text { thrin 1:40; Dynapact } 1 \%\end{array}$ \\
\hline $9 / 15-9 / 19 / 2008$ & NA & Drained fields \\
\hline $5 / 20 / 2008$ & NA & 2 pt. Glyphosate (generic) on N. Half \\
\hline $5 / 21 / 2008$ & NA & 2 pt. Glyphosate (generic) sequence on S. Half \\
\hline $6 / 15 / 2008$ & Irrigate (MRVA) & NA \\
\hline $6 / 23 / 2008$ & NA & Pyraclostrobin 4 oz. and Cyhalothrin 1:70 \\
\hline $10 / 2-10 / 4 / 2008$ & Harvest & NA \\
\hline \multicolumn{3}{|c|}{ TB33 } \\
\hline $2 / 25 / 2008$ & NA & $\begin{array}{l}\text { Glyphosate } 2 \text { pt. (generic); } 1.5 \text { pt. 2,4-dichlorophenoxy } \\
\text { acetic acid }\end{array}$ \\
\hline $4 / 2 / 2008$ & Plant & NA \\
\hline $4 / 25 / 2008$ & NA & 22 oz. Glyphosate (Roundup MAX) \\
\hline $5 / 25 / 2008$ & NA & 22 oz. Glyphosate (Roundup MAX) \\
\hline $6 / 8 / 2008$ & NA & Cyhalothrin 1:60; Quadrius $4 \mathrm{oz}$. \\
\hline $6 / 15 / 2008$ & Irrigate (MRVA) & NA \\
\hline $9 / 2-9 / 3 / 2008$ & Harvest; no till, ready to plant & NA \\
\hline
\end{tabular}


Table 7. Land managers' report of physical and chemical treatments of fields during crop production, Tommie Bayou Basin, Mississippi, 2008. - Continued

[NA, no treatment; oz, ounce; \#, pound; qt., quart; MRVA, Mississippi River valley aquifer; pt., pint; GPA, gallons per acre; \%, percent; see fig. 6 for locations]

\begin{tabular}{|c|c|c|}
\hline Date & Physical treatment & Chemical treatment \\
\hline \multicolumn{3}{|c|}{ TB34 } \\
\hline $11 / 1 / 2007$ & Plant wheat & NA \\
\hline $3 / 7 / 2008$ & NA & Urea $150 \#$ \\
\hline $6 / 8 / 2008$ & Harvest & NA \\
\hline $6 / 20 / 2008$ & NA & 2 pt. Glyphosate (generic) \\
\hline $6 / 25 / 2008$ & Irrigate (MRVA) & NA \\
\hline $7 / 10 / 2008$ & NA & 2 pt. Glyphosate (generic); Cyhalothrin 1:60; Azoxystrobin 4 oz. \\
\hline $8 / 15 / 2008$ & Irrigate (MRVA) & NA \\
\hline $9 / 2-9 / 3 / 2008$ & Harvest & NA \\
\hline $4 / 2 / 2008$ & Plant & NA \\
\hline $4 / 25 / 2008$ & NA & 22 oz. Glyphosate (Roundup MAX) \\
\hline $5 / 25 / 2008$ & NA & 22 oz. Glyphosate (Roundup MAX) \\
\hline $6 / 8 / 2008$ & NA & Cyhalothrin 1:60; Quadrius 4 oz. \\
\hline $6 / 15 / 2008$ & Irrigate (MRVA) & NA \\
\hline $9 / 2-9 / 3 / 2008$ & Harvest; no till, ready to plant & NA \\
\hline \multicolumn{3}{|c|}{ TB36 } \\
\hline $2 / 28 / 2008$ & NA & 2 pt. Glyphosate (generic) \\
\hline $4 / 16 / 2008$ & Planted & 22 oz. Glyphosate (Roundup UltraMAX) \\
\hline $10 / 8 / 2007$ & NA & Osprey 4.7 oz.; Thifensulfuron methyl $0.5 \mathrm{oz}$. \\
\hline $2 / 21 / 2008$ & NA & $125 \# 41.0 .0 .4$ \\
\hline $3 / 5 / 2008$ & NA & $100 \# 41.0 .0 .4$ \\
\hline $6 / 15 / 2008$ & Harvest; left fallow & NA \\
\hline \multicolumn{3}{|c|}{ TB38 } \\
\hline Between $2 / 25-2 / 28 / 2008$ & NA & 1 qt. Glyphosate (generic); Flumioxazin 1.5 oz. (herbicide) \\
\hline $4 / 1-4 / 4 / 2008$ & Disk, scratch, harrow & NA \\
\hline $4 / 20 / 2008$ & Plant & NA \\
\hline $5 / 7 / 2008$ & NA & Imazethapyr 4 oz. \\
\hline $5 / 17 / 2008$ & NA & Imazethapyr 4 oz.; Cyhalothrin 1:70 \\
\hline $6 / 1 / 2008$ & START FLOOD & 200\# 41.0.0.4; \\
\hline $6 / 15 / 2008$ & NA & 100\# 41.0.0.4 \\
\hline $7 / 1 / 2008$ & NA & $100 \# 41.0 .0 .4$ \\
\hline $9 / 1 / 2008$ & Drained field & NA \\
\hline $9 / 8-9 / 12 / 2008$ & Harvest; disk and harrow & NA \\
\hline
\end{tabular}


Table 7. Land managers' report of physical and chemical treatments of fields during crop production, Tommie Bayou Basin, Mississippi, 2008.-Continued

[NA, no treatment; oz, ounce; \#, pound; qt., quart; MRVA, Mississippi River valley aquifer; pt., pint; GPA, gallons per acre; \%, percent; see fig. 6 for locations]

\begin{tabular}{|c|c|c|}
\hline Date & Physical treatment & Chemical treatment \\
\hline \multicolumn{3}{|c|}{ TB39 } \\
\hline Between $2 / 25-2 / 28 / 2008$ & NA & 1 qt. Glyphosate (generic); Flumioxazin $1.5 \mathrm{oz}$. (herbicide) \\
\hline $5 / 25 / 2008$ & NA & 2 pt. Glyphosate (generic) \\
\hline $6 / 8 / 2008$ & NA & 1:60 Cyhalothrin; Azoxystrobin $5 \mathrm{oz}$. \\
\hline \multicolumn{3}{|c|}{ TB40 } \\
\hline $2 / 15 / 2008$ & NA & 2 pt. Glyphosate (generic) \\
\hline $5 / 21-5 / 22 / 2008$ & Plant & NA \\
\hline $6 / 6 / 2008$ & NA & Imazethapyr 6 oz. \\
\hline $6 / 21 / 2008$ & NA & Imazethapyr 6 oz. \\
\hline $7 / 15 / 2008$ & NA & $135 \#$ urea \\
\hline $8 / 9 / 2008$ & NA & Azoxystrobin 4 oz.; Cyhalothrin 1:70 \\
\hline $9 / 15-9 / 19 / 2008$ & Drained field & NA \\
\hline $9 / 29-10 / 3 / 2008$ & Harvest; disk twice and harrow & NA \\
\hline \multicolumn{3}{|c|}{ TB41 } \\
\hline $4 / 11 / 2008$ & Planted & NA \\
\hline $4 / 15 / 2008$ & NA & 23 oz. Glyphosate (generic); 5 GPA Flumioxazin 2 oz. \\
\hline $6 / 15 / 2008$ & Irrigated (MRVA) & NA \\
\hline $7 / 16 / 2008$ & NA & 23 oz. Glyphosate (generic); $12.8 \mathrm{oz}$. Blazer at $7.5 \mathrm{GPA}$ \\
\hline $8 / 1 / 2008$ & NA & $4.5 \mathrm{oz}$. Endigo 5 GPA (insecticide) \\
\hline $9 / 17 / 2008$ & Harvest & NA \\
\hline $10 / 8 / 2008$ & Field cultivated & NA \\
\hline $10 / 22 / 2008$ & Harrow & NA \\
\hline
\end{tabular}


Table 7. Land managers' report of physical and chemical treatments of fields during crop production, Tommie Bayou Basin, Mississippi, 2008. - Continued

[NA, no treatment; oz, ounce; \#, pound; qt., quart; MRVA, Mississippi River valley aquifer; pt., pint; GPA, gallons per acre; \%, percent; see fig. 6 for locations]

\begin{tabular}{|c|c|c|}
\hline Date & Physical treatment & Chemical treatment \\
\hline \multicolumn{3}{|c|}{ TB42 } \\
\hline $4 / 11 / 2008$ & Planted & NA \\
\hline $4 / 21 / 2008$ & NA & 1.5 pt. Glyphosate (generic) \\
\hline $5 / 19 / 2008$ & NA & 1.5 pt. Glyphosate (generic); 5 GPA Synchrony $.375 \mathrm{oz}$ \\
\hline $6 / 15 / 2008$ & Irrigated (MRVA) & NA \\
\hline $7 / 17 / 2008$ & NA & 23 oz. Glyphosate (generic); 12.8 Blazer at $7.5 \mathrm{GPA}$ \\
\hline $8 / 1 / 2008$ & NA & 4.5 oz. Endigo 5 GPA (insecticide) \\
\hline $9 / 17 / 2008$ & Harvest & NA \\
\hline $10 / 8 / 2008$ & Field cultivated & NA \\
\hline $10 / 22 / 2008$ & Harrow & NA \\
\hline \multicolumn{3}{|c|}{ TB43 } \\
\hline $4 / 11 / 2008$ & Planted & NA \\
\hline $4 / 15 / 2008$ & NA & 1.5 pt. Glyphosate (generic); Flumioxazin $1 / 5 \mathrm{oz} /$ acre \\
\hline $6 / 30 / 2008$ & Irrigate (MRVA) & NA \\
\hline $9 / 17 / 2008$ & Harvest & NA \\
\hline $10 / 8 / 2008$ & Field cultivated & NA \\
\hline $10 / 22 / 2008$ & Harrow & NA \\
\hline \multicolumn{3}{|c|}{ TB44 } \\
\hline \multicolumn{3}{|l|}{ Did not participate } \\
\hline \multicolumn{3}{|c|}{ TB45 } \\
\hline Did not participate & & \\
\hline
\end{tabular}


Table 8. Land managers' report of physical and chemical treatments of fields during crop production, unnamed tributary to Clear Creek Basin, Mississippi, 2007.

[NA, not applicable; gal, gallon; GW, groundwater; lbs, pounds; MRVA, Mississippi River valley aquifer; see fig. 7 for locations]

\begin{tabular}{|c|c|c|}
\hline Date & Physical treatment & Chemical treatment \\
\hline \multicolumn{3}{|c|}{ Field 2} \\
\hline $2 / 26 / 2007$ & NA & $\begin{array}{l}\text { Aerial application of a } 300 \text { gal load containing: Glyphosate (Roundup } \\
\text { WeatherMAX) 1:8 ratio, 2,4-dichlorophenoxy acetic acid 1:10 ratio, } \\
\text { petroleum distillate and alkyl phenol ethoxylate 1:30 ratio }\end{array}$ \\
\hline $4 / 4 / 2007$ & Planted crop & NA \\
\hline $4 / 9-4 / 13 / 2007$ & NA & $\begin{array}{l}\text { Using a ground rig applied a } 300 \text { gal load containing: 1:8 Glyphosate } \\
\text { (Roundup WeatherMAX), 1:8 Carfentrazone-ethyl }\end{array}$ \\
\hline $5 / 17-5 / 20 / 2007$ & Flooded field, GW & Aerial application of $200 \mathrm{lbs}$ urea \\
\hline $5 / 21-5 / 25 / 2007$ & $\begin{array}{l}\text { Pulled levees, put in levee gates using } \\
\text { levee squeezer and pusher }\end{array}$ & NA \\
\hline $6 / 8 / 2007$ & Mid-season flooding, GW & Aerial application of $100 \mathrm{lbs}$ urea \\
\hline $6 / 11-6 / 15 / 2007$ & NA & Aerial application of Bispyribac-sodium to western field edges \\
\hline $6 / 25-6 / 30 / 2007$ & NA & $\begin{array}{l}\text { Sprayed edges of field using a ground rig with a hooded sprayer: } \\
\text { 2,4-dichlorophenoxy acetic acid }\end{array}$ \\
\hline $7 / 30-8 / 3 / 2007$ & Drained water from field & NA \\
\hline 9/9/2007 & Harvested with combine & NA \\
\hline \multicolumn{3}{|c|}{ Field 3} \\
\hline $2 / 26 / 2007$ & NA & $\begin{array}{l}\text { Aerial application of a } 300 \text { gal load containing: Glyphosate (Roundup } \\
\text { WeatherMAX) 1:8 ratio, 2,4-dichlorophenoxy acetic acid 1:10 ratio, } \\
\text { petroleum distillate and alkyl phenol ethoxylate 1:30 ratio }\end{array}$ \\
\hline $4 / 4 / 2007$ & Planted crop & NA \\
\hline $4 / 9-4 / 13 / 2007$ & NA & $\begin{array}{l}\text { Using a ground rig applied a } 300 \text { gal load containing: 1:8 Glyphosate } \\
\text { (Roundup WeatherMAX), 1:8 Carfentrazone-ethyl }\end{array}$ \\
\hline $6 / 25-6 / 30 / 2007$ & NA & $\begin{array}{l}\text { Sprayed edges of field using a ground rig with a hooded sprayer: } \\
\text { 2,4-dichlorophenoxy acetic acid }\end{array}$ \\
\hline $7 / 30-8 / 3 / 2007$ & Drained water from field & NA \\
\hline 9/9/2007 & Harvested with combine & NA \\
\hline \multicolumn{3}{|c|}{ Field 4} \\
\hline $2 / 26 / 2007$ & NA & $\begin{array}{l}\text { Aerial application of a } 300 \text { gal load containing: Glyphosate (Roundup } \\
\text { WeatherMAX) 1:8 ratio; } 2,4 \text {-dichlorophenoxy acetic acid 1:10 ratio; } \\
\text { petroleum distillate and alkyl phenol ethoxylate } 1: 30 \text { ratio }\end{array}$ \\
\hline $4 / 4 / 2007$ & Planted crop & NA \\
\hline $4 / 9-4 / 13 / 2007$ & NA & $\begin{array}{l}\text { Using a ground rig applied a } 300 \text { gal load containing: 1:8 Glyphosate } \\
\text { (Roundup WeatherMAX), 1:8 Carfentrazone-ethyl }\end{array}$ \\
\hline $5 / 17-5 / 20 / 2007$ & Flooded field, GW & Aerial application of $200 \mathrm{lbs}$ urea \\
\hline
\end{tabular}


Table 8. Land managers' report of physical and chemical treatments of fields during crop production, unnamed tributary to Clear Creek Basin, Mississippi, 2007.-Continued

[NA, not applicable; gal, gallon; GW, groundwater; lbs, pounds; MRVA, Mississippi River valley aquifer; see fig. 7 for locations]

\begin{tabular}{|c|c|c|}
\hline Date & Physical treatment & Chemical treatment \\
\hline \multicolumn{3}{|c|}{ Field 4-Continued } \\
\hline $5 / 21-5 / 25 / 2007$ & $\begin{array}{l}\text { Pulled levees, put in levee gates using } \\
\text { levee squeezer and pusher }\end{array}$ & NA \\
\hline $6 / 8 / 2007$ & Mid-season flooding, GW & Aerial application of $100 \mathrm{lbs}$ urea \\
\hline $6 / 18-6 / 21 / 2007$ & Mid-season flooding, GW & $\begin{array}{l}\text { Aerial application of } 100 \mathrm{lbs} \text { urea; Ground rig application of a } \\
300 \text { gal load: Glyphosate (Roundup WeatherMAX), 2,4-dichloro- } \\
\text { phenoxy acetic acid sprayed on levees in 1:8 ratio }\end{array}$ \\
\hline $6 / 25-6 / 30 / 2007$ & NA & $\begin{array}{l}\text { Sprayed edges of field using a ground rig with a hooded sprayer: } \\
\text { 2,4-dichlorophenoxy acetic acid }\end{array}$ \\
\hline $7 / 30-8 / 3 / 2007$ & Drained water from field & NA \\
\hline $9 / 12 / 2007$ & Harvested with combine & NA \\
\hline \multicolumn{3}{|c|}{ Field 8} \\
\hline $3 / 20 / 2007$ & Tilled with field cultivator & NA \\
\hline $3 / 26-3 / 30 / 2007$ & Harrowed & NA \\
\hline $4 / 30 / 2007$ & Planted crop & NA \\
\hline $5 / 7-5 / 11 / 2007$ & NA & $\begin{array}{l}\text { Using a ground rig with a hooded sprayer applied a } 300 \text { gal load } \\
\text { containing: Glyphosate (Roundup WeatherMAX) in a 1:4 ratio, } \\
\text { FirstRate in a 1:6.66 ratio }\end{array}$ \\
\hline $5 / 16 / 2007$ & Replanted & NA \\
\hline $6 / 25-6 / 30 / 2007$ & NA & $\begin{array}{l}\text { Sprayed edges of field using a ground rig with a hooded sprayer: } \\
\text { 2,4-dichlorophenoxy acetic acid }\end{array}$ \\
\hline $2 / 26 / 2007$ & NA & $\begin{array}{l}\text { Aerial application of a } 300 \text { gal load containing: Glyphosate (Roundup } \\
\text { WeatherMAX) 1:8 ratio; 2,4-dichlorophenoxy acetic acid 1:10 ratio; } \\
\text { petroleum distillate and alkyl phenol ethoxylate 1:30 ratio }\end{array}$ \\
\hline $4 / 30 / 2007$ & Planted crop & NA \\
\hline $5 / 7-5 / 11 / 2007$ & NA & $\begin{array}{l}\text { Using a ground rig with a hooded sprayer applied a } 300 \text { gal load } \\
\text { containing: Glyphosate (Roundup WeatherMAX) in a 1:4 ratio, } \\
\text { FirstRate in a 1:6.66 ratio }\end{array}$ \\
\hline $6 / 1 / 2007$ & $\begin{array}{l}\text { Irrigated (precision flush-on-field) } \\
\text { source MRVA; } 41 \text { hours }\end{array}$ & NA \\
\hline $6 / 25-6 / 30 / 2007$ & NA & $\begin{array}{l}\text { Sprayed edges of field using a ground rig with a hooded sprayer: } \\
\text { 2,4-dichlorophenoxy acetic acid }\end{array}$ \\
\hline $7 / 23-7 / 27 / 2007$ & NA & $\begin{array}{l}\text { Ground rig, hooded sprayer 5:1, } 300 \text { gal load: Glyphosate (Roundup } \\
\text { WeatherMAX), Quadrant 1:8 }\end{array}$ \\
\hline $7 / 30-8 / 3 / 2007$ & $\begin{array}{l}\text { Irrigated (precision flush-on-field) } \\
\text { source MRVA }\end{array}$ & NA \\
\hline $10 / 3-10 / 4 / 2007$ & Harvested with combine & NA \\
\hline
\end{tabular}


Table 8. Land managers' report of physical and chemical treatments of fields during crop production, unnamed tributary to Clear Creek Basin, Mississippi, 2007.-Continued

[NA, not applicable; gal, gallon; GW, groundwater; lbs, pounds; MRVA, Mississippi River valley aquifer; see fig. 7 for locations]

\begin{tabular}{|c|c|c|}
\hline Date & Physical treatment & Chemical treatment \\
\hline \multicolumn{3}{|c|}{ Field 10} \\
\hline $2 / 26 / 2007$ & NA & $\begin{array}{l}\text { Aerial application of a } 300 \text { gal load containing: Glyphosate (Roundup } \\
\text { WeatherMAX) 1:8 ratio, 2,4-dichlorophenoxy acetic acid 1:10 ratio, } \\
\text { petroleum distillate and alkyl phenol ethoxylate } 1: 30 \text { ratio }\end{array}$ \\
\hline $4 / 30 / 2007$ & Planted crop & NA \\
\hline $5 / 7-5 / 11 / 2007$ & NA & $\begin{array}{l}\text { Using a ground rig with a hooded sprayer applied a } 300 \text { gal load } \\
\text { containing: Glyphosate (Roundup WeatherMAX) in a 1:4 ratio, } \\
\text { FirstRate in a 1:6.66 ratio }\end{array}$ \\
\hline $6 / 1 / 2007$ & $\begin{array}{l}\text { Irrigated (precision flush-on-field) } \\
\text { source MRVA; } 36 \text { hours }\end{array}$ & NA \\
\hline $6 / 25-6 / 30 / 2007$ & NA & $\begin{array}{l}\text { Sprayed edges of field using a ground rig with a hooded sprayer: } \\
\text { 2,4-dichlorophenoxy acetic acid }\end{array}$ \\
\hline $10 / 3-10 / 4 / 2007$ & Harvested with combine & NA \\
\hline
\end{tabular}

Field 14S

\begin{tabular}{|c|c|c|}
\hline $2 / 27 / 2007$ & NA & $\begin{array}{l}\text { Aerial application of a } 300 \text { gal load containing: Glyphosate (Roundup } \\
\text { WeatherMAX) 1:8 ratio, 2,4-dichlorophenoxy acetic acid 1:10 ratio, } \\
\text { petroleum distillate and alkyl phenol ethoxylate } 1: 30 \text { ratio }\end{array}$ \\
\hline $4 / 21 / 2007$ & Planted crop & NA \\
\hline $5 / 7-5 / 11 / 2007$ & NA & $\begin{array}{l}\text { Using a ground rig with a hooded sprayer applied a } 300 \text { gal load } \\
\text { containing: Glyphosate (Roundup WeatherMAX) in a 1:4 ratio, } \\
\text { FirstRate in a 1:6.66 ratio }\end{array}$ \\
\hline $6 / 6 / 2007$ & $\begin{array}{l}\text { Irrigated (precision flush-on-field) } \\
\text { source MRVA }\end{array}$ & NA \\
\hline $6 / 25-6 / 30 / 2007$ & NA & $\begin{array}{l}\text { Sprayed edges of field using a ground rig with a hooded sprayer: } \\
\text { 2,4-dichlorophenoxy acetic acid }\end{array}$ \\
\hline $7 / 30-8 / 3 / 2007$ & $\begin{array}{l}\text { Irrigated (precision flush-on-field) } \\
\text { source MRVA }\end{array}$ & NA \\
\hline $9 / 18-9 / 19 / 2007$ & Harvested with combine & NA \\
\hline \multicolumn{3}{|c|}{ Field $14 \mathrm{~N}$} \\
\hline $2 / 27 / 2007$ & NA & $\begin{array}{l}\text { Aerial application of a } 300 \text { gal load containing: Glyphosate (Roundup } \\
\text { WeatherMAX) 1:8 ratio, 2,4-dichlorophenoxy acetic acid 1:10 ratio, } \\
\text { petroleum distillate and alkyl phenol ethoxylate 1:30 ratio }\end{array}$ \\
\hline
\end{tabular}

$\begin{array}{ll}4 / 5-4 / 6 / 2007 & \text { Planted crop } \\ 4 / 9-4 / 13 / 2007 & \text { NA } \\ 5 / 17-5 / 20 / 2007 & \text { Flooded field, GW } \\ 5 / 21-5 / 25 / 2007 & \begin{array}{c}\text { Pulled levees, put in levee gates using } \\ \text { levee squeezer and pusher }\end{array} \\ \text { 6/8/2007 } & \text { Mid-season flooding, GW } \\ 6 / 18-6 / 21 / 2007 & \text { Mid-season flooding, GW }\end{array}$

Using a ground rig applied a 300 gal load containing: 1:8 Glyphosate (Roundup WeatherMAX), 1:8 Carfentrazone-ethyl

Aerial application of 200 lbs urea

NA

Aerial application of $100 \mathrm{lbs}$ urea

Aerial application of $100 \mathrm{lbs}$ urea; Ground rig application of a 300 gal load: Glyphosate (Roundup WeatherMAX), 2,4-dichlorophenoxy acetic acid sprayed on levees in 1:8 ratio 
Table 8. Land managers' report of physical and chemical treatments of fields during crop production, unnamed tributary to Clear Creek Basin, Mississippi, 2007.-Continued

[NA, not applicable; gal, gallon; GW, groundwater; lbs, pounds; MRVA, Mississippi River valley aquifer; see fig. 7 for locations]

\begin{tabular}{|c|c|c|}
\hline Date & Physical treatment & Chemical treatment \\
\hline \multicolumn{3}{|c|}{ Field $14 \mathrm{~N}$-Continued } \\
\hline $6 / 25-6 / 30 / 2007$ & NA & $\begin{array}{l}\text { Sprayed edges of field using a ground rig with a hooded sprayer: } \\
\text { 2,4-dichlorophenoxy acetic acid }\end{array}$ \\
\hline $7 / 30-8 / 3 / 2007$ & Drained water from field & NA \\
\hline $9 / 15-9 / 16 / 2007$ & Harvested with combine & NA \\
\hline $4 / 5-4 / 6 / 2007$ & Planted crop & NA \\
\hline $4 / 9-4 / 13 / 2007$ & NA & $\begin{array}{l}\text { Using a ground rig applied a } 300 \text { gal load containing: 1:8 Glyphosate } \\
\text { (Roundup WeatherMAX), 1:8 Carfentrazone-ethyl }\end{array}$ \\
\hline $6 / 8 / 2007$ & Mid-season flooding, GW & Aerial application of $100 \mathrm{lbs}$ urea \\
\hline $6 / 18-6 / 21 / 2007$ & Mid-season flooding, GW & $\begin{array}{l}\text { Aerial application of } 100 \mathrm{lbs} \text { urea; Ground rig application } \\
\text { of a } 300 \text { gal load: Glyphosate (Roundup WeatherMAX), } \\
\text { 2,4-dichlorophenoxy acetic acid sprayed on levees in 1:8 ratio }\end{array}$ \\
\hline $6 / 25-6 / 30 / 2007$ & NA & $\begin{array}{l}\text { Sprayed edges of field using a ground rig with a hooded sprayer: } \\
\text { 2,4-dichlorophenoxy acetic acid }\end{array}$ \\
\hline $7 / 30-8 / 3 / 2007$ & Drained water from field & NA \\
\hline 9/9/2007 & Harvested with combine & NA \\
\hline \multicolumn{3}{|c|}{ Field 16} \\
\hline $6 / 13-6 / 14 / 2007$ & $\begin{array}{l}\text { Irrigated (precision flush-on-field) source } \\
\text { MRVA the West and East halves; } \\
\text { Tractor East half, creating furrs to let } \\
\text { water run down East half }\end{array}$ & NA \\
\hline $6 / 25-6 / 30 / 2007$ & NA & $\begin{array}{l}\text { Sprayed edges of field using a ground rig with a hooded sprayer: } \\
\text { 2,4-dichlorophenoxy acetic acid }\end{array}$ \\
\hline $7 / 30-8 / 3 / 2007$ & $\begin{array}{l}\text { Irrigated (precision flush-on-field) } \\
\text { source MRVA }\end{array}$ & NA \\
\hline $9 / 19-9 / 20 / 2007$ & Harvested with combine & NA \\
\hline
\end{tabular}


Table 9. Land managers' report of physical and chemical treatments of fields during crop production, unnamed tributary to Clear Creek Basin, Mississippi, 2008.

[NA, no treatment; ratio represents gallon of pesticide/herbicide per acre; oz, ounce; pt, pint; qt, quart; USGS, U.S. Geological Survey; \%, percent; hrs, hours; MRVA, Mississippi River valley aquifer; see fig. 8 for locations]

\begin{tabular}{|c|c|c|}
\hline Date & Physical treatment & Chemical treatment \\
\hline \multicolumn{3}{|c|}{ Field 21} \\
\hline $2 / 4-2 / 8 / 2008$ & $\begin{array}{l}\text { Main drainage ditch near the USGS gage, } \\
\text { unnamed tributary to Clear Creek near } \\
\text { Napanee, MS, cleaned out and culverts replaced }\end{array}$ & NA \\
\hline $2 / 15 / 2008$ & NA & $\begin{array}{l}\text { Aerial application of } 100 \text { acre load containing: Glyphosate } \\
\text { (Roundup PowerMAX) } 22 \text { oz/acre; 2,4-dichlorophenoxy } \\
\text { acetic acid } 1.5 \mathrm{pt} / \text { acre }\end{array}$ \\
\hline $3 / 17-3 / 21 / 2008$ & $\begin{array}{l}\text { Ditches that run east and west of the USGS gage, } \\
\text { unnamed tributary to Clear Creek near } \\
\text { Napanee, MS, were cleaned out using a tractor }\end{array}$ & NA \\
\hline $3 / 24-3 / 28 / 2008$ & $\begin{array}{l}\text { Fields planted in rice during } 2007 \text { were } \\
\text { disked across rice levees with tractor and } \\
\text { planed to smooth }\end{array}$ & NA \\
\hline $6 / ? / 2008$ & $\begin{array}{l}\text { Plowed furrows for irrigation trench, laid } \\
\text { irrigation pipe }\end{array}$ & NA \\
\hline $7 / 4-7 / 6 / 2008$ & $\begin{array}{l}\text { All fields irrigated; turned on pump } \\
\text { for } 48 \mathrm{hrs} \text { (MRVA) }\end{array}$ & NA \\
\hline $7 / 7-7 / 11 / 2008$ & NA & Using a ground-rig applied Glyphosate (generic) 1 qt/acre \\
\hline $10 / 27-10 / 23 / 2008$ & $\begin{array}{l}\text { Harvested; fields disked and cultivated; } \\
\text { rows reformed }\end{array}$ & NA \\
\hline
\end{tabular}

\begin{tabular}{lll}
\hline \multicolumn{3}{c}{ Field 20} \\
\hline $2 / 4-2 / 8 / 2008$ & Main drainage ditch near the USGS gage, & NA
\end{tabular}
unnamed tributary to Clear Creek near Napanee, MS, dug out and culverts replaced

$2 / 15 / 2008$

$3 / 17-3 / 21 / 2008$

$3 / 24-3 / 28 / 2008$

$6 / 2-6 / 5 / 2008$

$6 / 9-6 / 13 / 2008$

6/?/2008

$7 / 4-7 / 6 / 2008$

$7 / 7-7 / 11 / 2008$

$10 / 27-10 / 23 / 2008$
NA

Two ditches that run east and west of the USGS gage, unnamed tributary to Clear Creek near Napanee, MS, were cleaned out with tractor

2007 rice fields: Disked across rice levees with tractor and land plane to smooth out land; will make planting easier

Soybeans: All soy fields planted

NA

All fields: Plowed out furrows to make trench for irrigation water. Laid out irrigation pipe

All fields irrigated; turned on pump for 48 hrs (MRVA)

NA

Harvest all soybeans; disked and cultivated fields to smooth out and reform rows
Glyphosate (Roundup PowerMAX) 22 oz/acre; 2,4-dichlorophenoxy acetic acid $1.5 \mathrm{pt} / \mathrm{acre}$, applied by plane; 100 acre load

NA

NA

NA

Glyphosate (generic) and Metolachlor (Fomesafen sodium $\&$ dual) sprayed 1 qt/acre (50 acres a load) used ground-rig; all fields

NA

NA

Glyphosate (generic) $1 \mathrm{qt} /$ acre applied to all fields with ground-rig

NA 
Table 9. Land managers' report of physical and chemical treatments of fields during crop production, unnamed tributary to Clear Creek Basin, Mississippi, 2008.-Continued

[NA, no treatment; ratio represents gallon of pesticide/herbicide per acre; oz, ounce; pt, pint; qt, quart; USGS, U.S. Geological Survey; \%, percent; hrs, hours; MRVA, Mississippi River valley aquifer; see fig. 8 for locations]

\begin{tabular}{|c|c|c|}
\hline Date & Physical treatment & Chemical treatment \\
\hline \multicolumn{3}{|c|}{ Field 19} \\
\hline $2 / 4-2 / 8 / 2008$ & $\begin{array}{l}\text { Main drainage ditch near the USGS gage, } \\
\text { unnamed tributary to Clear Creek near } \\
\text { Napanee, MS, dug out and culverts replaced }\end{array}$ & NA \\
\hline $2 / 15 / 2008$ & NA & $\begin{array}{l}\text { Glyphosate (Roundup PowerMAX) } 22 \text { oz/acre; } 2,4 \text {-dichloro- } \\
\text { phenoxy acetic acid } 1.5 \text { pt/acre, applied by plane; } 100 \text { acre load }\end{array}$ \\
\hline $3 / 24-3 / 28 / 2008$ & $\begin{array}{l}2007 \text { rice fields: Disked across rice levees } \\
\text { with tractor and land plane to smooth out land; } \\
\text { will make planting easier }\end{array}$ & NA \\
\hline $6 / ? / 2008$ & $\begin{array}{l}\text { All fields: Plowed out furrows to make trench } \\
\text { for irrigation water. Laid out irrigation pipe }\end{array}$ & NA \\
\hline $7 / 4-7 / 6 / 2008$ & $\begin{array}{l}\text { All fields irrigated; turned on pump for } \\
48 \text { hrs (MRVA) }\end{array}$ & NA \\
\hline $7 / 7-7 / 11 / 2008$ & NA & Glyphosate (generic) $1 \mathrm{qt} /$ acre applied to all fields with ground-rig \\
\hline $10 / 27-10 / 23 / 2008$ & $\begin{array}{l}\text { Harvest all soybeans; disked and cultivated fields } \\
\text { to smooth out and reform rows }\end{array}$ & NA \\
\hline \multicolumn{3}{|c|}{ Field 15} \\
\hline $2 / 4-2 / 8 / 2008$ & $\begin{array}{l}\text { Main drainage ditch near the USGS gage, } \\
\text { unnamed tributary to Clear Creek near } \\
\text { Napanee, MS, dug out and culverts replaced }\end{array}$ & NA \\
\hline $6 / 2-6 / 5 / 2008$ & Soybeans: All soy fields planted & NA \\
\hline $6 / 9-6 / 13 / 2008$ & NA & $\begin{array}{l}\text { Glyphosate (generic) and Metolachlor (Fomesafen sodium and dual) } \\
\text { sprayed } 1 \text { qt/acre ( } 50 \text { acres a load) used ground-rig; all fields }\end{array}$ \\
\hline $6 / ? / 2008$ & $\begin{array}{l}\text { All fields: Plowed out furrows to make trench } \\
\text { for irrigation water. Laid out irrigation pipe }\end{array}$ & NA \\
\hline $7 / 4-7 / 6 / 2008$ & $\begin{array}{l}\text { All fields irrigated; turned on pump } \\
\text { for } 48 \mathrm{hrs} \text { (MRVA) }\end{array}$ & NA \\
\hline $7 / 7-7 / 11 / 2008$ & NA & Glyphosate (generic) $1 \mathrm{qt} / \mathrm{acre}$ applied to all fields with ground-rig \\
\hline $7 / 22 / 2008$ & NA & $\begin{array}{l}\text { Sprayed Glyphosate (generic) } 1 \text { qt/acre, } 50 \text { acre load; } \\
\text { with ground-rig }\end{array}$ \\
\hline $7 / 31 / 2008$ & Irrigated; turned on pump for $48 \mathrm{hrs}$ (MRVA) & NA \\
\hline $10 / 27-10 / 23 / 2008$ & $\begin{array}{l}\text { Harvest all soybeans; disked and cultivated fields } \\
\text { to smooth out and reform rows }\end{array}$ & NA \\
\hline
\end{tabular}


Table 9. Land managers' report of physical and chemical treatments of fields during crop production, unnamed tributary to Clear Creek Basin, Mississippi, 2008. - Continued

[NA, no treatment; ratio represents gallon of pesticide/herbicide per acre; oz, ounce; pt, pint; qt, quart; USGS, U.S. Geological Survey; \%, percent; hrs, hours; MRVA, Mississippi River valley aquifer; see fig. 8 for locations]

\begin{tabular}{|c|c|c|}
\hline Date & Physical treatment & Chemical treatment \\
\hline \multicolumn{3}{|c|}{ Field 14} \\
\hline $2 / 4-2 / 8 / 2008$ & $\begin{array}{l}\text { Main drainage ditch near the USGS gage, } \\
\text { unnamed tributary to Clear Creek near } \\
\text { Napanee, MS, dug out and culverts replaced }\end{array}$ & NA \\
\hline $2 / 15 / 2008$ & NA & $\begin{array}{l}\text { Glyphosate (Roundup PowerMAX) } 22 \text { oz/acre; } 2,4 \text {-dichloro- } \\
\text { phenoxy acetic acid } 1.5 \text { pt/acre, applied by plane; } 100 \text { acre load }\end{array}$ \\
\hline $3 / 24-3 / 28 / 2008$ & $\begin{array}{l}2007 \text { rice fields: Disked across rice levees with } \\
\text { tractor and land plane to smooth out land; } \\
\text { will make planting easier }\end{array}$ & NA \\
\hline 6/?/2008 & $\begin{array}{l}\text { All fields: Plowed out furrows to make trench } \\
\text { for irrigation water. Laid out irrigation pipe }\end{array}$ & NA \\
\hline $7 / 4-7 / 6 / 2008$ & $\begin{array}{l}\text { All fields irrigated; turned on pump for } \\
48 \text { hrs (MRVA) }\end{array}$ & NA \\
\hline $7 / 7-7 / 11 / 2008$ & NA & $\begin{array}{l}\text { Glyphosate (generic) } 1 \mathrm{qt} / \text { acre applied to all fields with } \\
\text { ground-rig }\end{array}$ \\
\hline $10 / 27-10 / 23 / 2008$ & $\begin{array}{l}\text { Harvest all soybeans; disked and cultivated } \\
\text { fields to smooth out and reform rows }\end{array}$ & NA \\
\hline
\end{tabular}

$2 / 4-2 / 8 / 2008$

$2 / 15 / 2008$

$3 / 17-3 / 21 / 2008$

$3 / 24-3 / 28 / 2008$

$6 / 2-6 / 5 / 2008$

$6 / 9-6 / 13 / 2008$

$6 / ? / 2008$

$7 / 4-7 / 6 / 2008$

$7 / 7-7 / 11 / 2008$

$10 / 27-10 / 23 / 2008$
Main drainage ditch near the USGS gage, unnamed tributary to Clear Creek near Napanee, MS, dug out and culverts replaced NA

Two ditches that run east and west of the USGS gage, unnamed tributary to Clear Creek near Napanee, MS, were cleaned out with tractor

2007 rice fields: Disked across rice levees with tractor and land plane to smooth out land; will make planting easier

Soybeans: All soy fields planted

NA for irrigation water. Laid out irrigation pipe

All fields irrigated; turned on pump for 48 hrs (MRVA)

NA

Harvest all soybeans; disked and cultivated fields to smooth out and reform rows
NA

Glyphosate (Roundup PowerMAX) 22 oz/acre; 2,4-dichlorophenoxy acetic acid $1.5 \mathrm{pt} / \mathrm{acre}$, applied by plane; $100 \mathrm{acre}$ load NA

NA

NA

Glyphosate (generic) and Metolachlor (Fomesafen sodium and dual) sprayed $1 \mathrm{qt} /$ acre (50 acres a load) used groundrig; all fields

NA

NA

Glyphosate (generic) $1 \mathrm{qt} /$ acre applied to all fields with ground-rig

NA 
Table 9. Land managers' report of physical and chemical treatments of fields during crop production, unnamed tributary to Clear Creek Basin, Mississippi, 2008.-Continued

[NA, no treatment; ratio represents gallon of pesticide/herbicide per acre; oz, ounce; pt, pint; qt, quart; USGS, U.S. Geological Survey; \%, percent; hrs, hours; MRVA, Mississippi River valley aquifer; see fig. 8 for locations]

\begin{tabular}{|c|c|c|}
\hline Date & Physical treatment & Chemical treatment \\
\hline \multicolumn{3}{|c|}{ Field 9} \\
\hline $2 / 4-2 / 8 / 2008$ & $\begin{array}{l}\text { Main drainage ditch near the USGS gage, } \\
\text { unnamed tributary to Clear Creek near } \\
\text { Napanee, MS, dug out and culverts replaced }\end{array}$ & NA \\
\hline $2 / 15 / 2008$ & NA & $\begin{array}{l}\text { Glyphosate (Roundup PowerMAX) } 22 \text { oz/acre; } 2,4 \text {-dichloro- } \\
\text { phenoxy acetic acid } 1.5 \text { pt/acre, applied by plane; } 100 \text {-acre load }\end{array}$ \\
\hline $3 / 24-3 / 28 / 2008$ & $\begin{array}{l}2007 \text { rice fields: Disked across rice levees with } \\
\text { tractor and land plane to smooth out land; } \\
\text { will make planting easier }\end{array}$ & NA \\
\hline $6 / 2-6 / 5 / 2008$ & Soybeans: All soy fields planted & NA \\
\hline $6 / ? / 2008$ & $\begin{array}{l}\text { All fields: Plowed out furrows to make trench } \\
\text { for irrigation water. Laid out irrigation pipe }\end{array}$ & NA \\
\hline $7 / 4-7 / 6 / 2008$ & $\begin{array}{l}\text { All fields irrigated; turned on pump for } \\
48 \mathrm{hrs} \text { (MRVA) }\end{array}$ & NA \\
\hline $7 / 7-7 / 11 / 2008$ & NA & Glyphosate (generic) 1 qt/acre applied to all fields with ground-rig \\
\hline $7 / 22 / 2008$ & NA & $\begin{array}{l}\text { Sprayed Glyphosate (generic) } 1 \text { qt/acre, } 50 \text {-acre load; with } \\
\text { ground-rig }\end{array}$ \\
\hline $10 / 27-10 / 23 / 2008$ & $\begin{array}{l}\text { Harvest all soybeans; disked and cultivated } \\
\text { fields to smooth out and reform rows }\end{array}$ & NA \\
\hline
\end{tabular}

\begin{tabular}{|c|c|c|}
\hline \multicolumn{3}{|c|}{ Field 8} \\
\hline $2 / 4-2 / 8 / 2008$ & $\begin{array}{l}\text { Main drainage ditch near the USGS gage, } \\
\text { unnamed tributary to Clear Creek near } \\
\text { Napanee, MS, dug out and culverts replaced }\end{array}$ & NA \\
\hline $2 / 15 / 2008$ & NA & $\begin{array}{l}\text { Glyphosate (Roundup PowerMAX) } 22 \text { oz/acre; } 2,4 \text {-dichloro- } \\
\text { phenoxy acetic acid } 1.5 \text { pt/acre, applied by plane; } 100 \text {-acre load }\end{array}$ \\
\hline $3 / 17-3 / 21 / 2008$ & $\begin{array}{l}\text { Two ditches that run East and west of the USGS } \\
\text { gage, unnamed tributary to Clear Creek near } \\
\text { Napanee, MS, were cleaned out with tractor }\end{array}$ & NA \\
\hline $3 / 24-3 / 28 / 2008$ & $\begin{array}{l}2007 \text { rice fields: Disked across rice levees with } \\
\text { tractor and land plane to smooth out land; } \\
\text { will make planting easier }\end{array}$ & NA \\
\hline $6 / 2-6 / 5 / 2008$ & Soybeans: All soy fields planted & NA \\
\hline $6 / 9-6 / 13 / 2008$ & NA & $\begin{array}{l}\text { Glyphosate (generic) and Metolachlor (Fomesafen sodium } \\
\text { and dual) sprayed } 1 \text { qt/acre ( } 50 \text { acres a load) used ground- } \\
\text { rig; all fields }\end{array}$ \\
\hline $7 / 4-7 / 6 / 2008$ & $\begin{array}{l}\text { All fields irrigated; turned on pump for } \\
48 \text { hrs (MRVA) }\end{array}$ & NA \\
\hline $7 / 7-7 / 11 / 2008$ & NA & Glyphosate (generic) 1 qt/acre applied to all fields with ground-rig \\
\hline $7 / 28-7 / 31 / 2008$ & NA & $\begin{array}{l}\text { Glyphosate (Roundup PowerMAX) } 1 \text { qt/acre w/ ground-rig } \\
\text { second application }\end{array}$ \\
\hline $10 / 27-10 / 23 / 2008$ & $\begin{array}{l}\text { Harvest all soybeans; disked and cultivated } \\
\text { fields to smooth out and reform rows }\end{array}$ & NA \\
\hline
\end{tabular}


Table 9. Land managers' report of physical and chemical treatments of fields during crop production, unnamed tributary to Clear Creek Basin, Mississippi, 2008.—Continued

[NA, no treatment; ratio represents gallon of pesticide/herbicide per acre; oz, ounce; pt, pint; qt, quart; USGS, U.S. Geological Survey; \%, percent; hrs, hours; MRVA, Mississippi River valley aquifer; see fig. 8 for locations]

\begin{tabular}{|c|c|c|}
\hline Date & Physical treatment & Chemical treatment \\
\hline \multicolumn{3}{|c|}{ Field 6} \\
\hline $2 / 4-2 / 8 / 2008$ & $\begin{array}{l}\text { Main drainage ditch near the USGS gage, } \\
\text { unnamed tributary to Clear Creek near } \\
\text { Napanee, MS, dug out and culverts replaced }\end{array}$ & NA \\
\hline $2 / 15 / 2008$ & NA & $\begin{array}{l}\text { Glyphosate (Roundup PowerMAX) } 22 \text { oz/acre; } 2,4 \text {-dichloro- } \\
\text { phenoxy acetic acid } 1.5 \text { pt/acre, applied by plane; } 100 \text {-acre load }\end{array}$ \\
\hline $3 / 24-3 / 28 / 2008$ & $\begin{array}{l}2007 \text { rice fields: Disked across rice levees with } \\
\text { tractor and land plane to smooth out land; } \\
\text { will make planting easier }\end{array}$ & NA \\
\hline $6 / ? / 2008$ & $\begin{array}{l}\text { All fields: Plowed out furrows to make trench } \\
\text { for irrigation water. Laid out irrigation pipe }\end{array}$ & NA \\
\hline $7 / 4-7 / 6 / 2008$ & $\begin{array}{l}\text { All fields irrigated; turned on pump for } \\
48 \mathrm{hrs} \text { (MRVA) }\end{array}$ & NA \\
\hline $7 / 7-7 / 11 / 2008$ & NA & $\begin{array}{l}\text { Glyphosate (generic) } 1 \mathrm{qt} / \text { acre applied to all fields with } \\
\text { ground-rig }\end{array}$ \\
\hline $10 / 27-10 / 23 / 2008$ & $\begin{array}{l}\text { Harvest all soybeans; disked and cultivated } \\
\text { fields to smooth out and reform rows }\end{array}$ & NA \\
\hline \multicolumn{3}{|c|}{ Field 7} \\
\hline $3 / 24-3 / 28 / 2008$ & $\begin{array}{l}2007 \text { rice fields: Disked across rice levees with } \\
\text { tractor and land plane to smooth out land; } \\
\text { will make planting easier }\end{array}$ & NA \\
\hline $6 / 2-6 / 5 / 2008$ & Soybeans: All soy fields planted & NA \\
\hline $6 / 9-6 / 13 / 2008$ & NA & $\begin{array}{l}\text { Glyphosate (generic) and Metolachlor (Fomesafen sodium } \\
\text { and dual) sprayed } 1 \text { qt/acre ( } 50 \text { acres a load) used ground- } \\
\text { rig; all fields }\end{array}$ \\
\hline $6 / ? / 2008$ & $\begin{array}{l}\text { All fields: Plowed out furrows to make trench } \\
\text { for irrigation water. Laid out irrigation pipe }\end{array}$ & NA \\
\hline $7 / 4-7 / 6 / 2008$ & $\begin{array}{l}\text { All fields irrigated; turned on pump for } \\
48 \mathrm{hrs} \text { (MRVA) }\end{array}$ & NA \\
\hline $7 / 7-7 / 11 / 2008$ & NA & $\begin{array}{l}\text { Glyphosate (generic) } 1 \mathrm{qt} / \text { acre applied to all fields with } \\
\text { ground-rig }\end{array}$ \\
\hline $10 / 27-10 / 23 / 2008$ & $\begin{array}{l}\text { Harvest all soybeans; disked and cultivated } \\
\text { fields to smooth out and reform rows }\end{array}$ & NA \\
\hline
\end{tabular}


Manuscript approved for publication, October 28, 2010

Edited by Rebecca J. Deckard

Illustrations and layout by Caryl J. Wipperfurth

For more information concerning the research in this report, contact

Richard H. Coupe

USGS Mississippi Water Science Center

NAWQA Mississippi Embayment Study Unit

308 South Airport Road

Pearl, Mississippi 39208-6649

telephone: 601-933-2982

http://water.usgs.gov/nawqa/ 
을. 